\title{
Thermal Stresses in Coatings on Carbon-Carbon Composites
}

\author{
by \\ Andreas W. Obst \\ Dissertation submitted to the faculty of the \\ Virginia Polytechnic Institute and State University \\ in partial fulfillment of the requirements for the degree of \\ DOCTOR OF PHILOSOPHY
}

in

Engineering Mechanics

APPROVED:

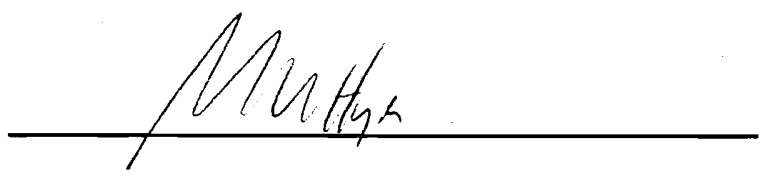

Committee Chair: Michael W. Hyer

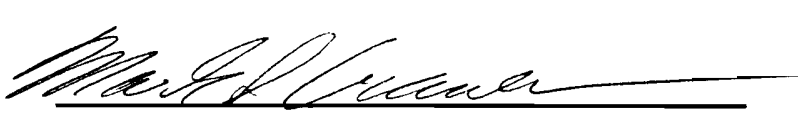

Mark S. Cramer

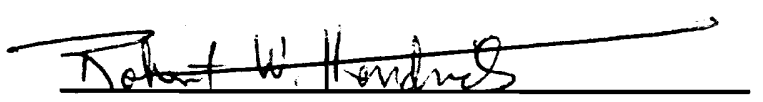

Robert W. Hendricks
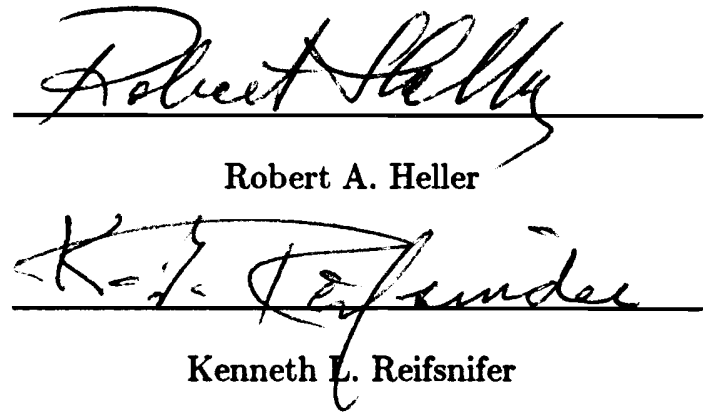

April, 1995

Blacksburg, Virginia 
c. 2

$$
\begin{gathered}
2 D \\
5655 \\
\text { V856 } \\
1995 \\
0278 \\
C .2
\end{gathered}
$$




\title{
Thermal Stresses in Coatings on Carbon-Carbon Composites
}

\author{
by \\ Andreas W. Obst \\ Committee Chair: Michael W. Hyer \\ Engineering Mechanics
}

\begin{abstract}
(ABSTRACT)
The objective of this study was to investigate thermal stresses in oxidation protection coatings on carbon-carbon composites. Multilayer coatings with each layer of coating applied at a different temperature, and gradient coatings with continuously varying properties through the thickness of the coating were considered. Particular emphasis was on the prediction of the thermal stresses in the vicinity of geometric discontinuities.

For the analysis an incremental generalized plane-strain finite element model that accounts for temperature-dependent material properties and continuously varying properties in the gradient coatings was developed. The model is based on an incremental constitutive equation for linear thermoelastic materials that accounts for the coupling between stresses and the temperature-dependence of the material properties. In addition to the finite element model, an incremental simplified plane stress analysis for the prediction of stresses away from geometric discontinuities was developed.

Analyses of carbon-carbon substrates with coatings showed that large stress concentrations in the coatings may be present near the geometric discontinuities. It was found that inserting a compliant layer between the carbon-carbon substrate and the oxidation protection coatings, or inserting a gradient coating with properties varying from those of a compliant material near the carbon-carbon substrate to those of the oxidation protection coating near the oxidation protection coating, could be used to significantly reduce the magnitudes of the stresses in the stress concentrations. The influence of geometric and material parameters on the stresses was studied and for some combinations of parameters stresses near the geometric discontinuities could be reduced to magnitudes that were smaller than
\end{abstract}


the magnitudes of the stresses away from the discontinuities.

For coatings applied at different temperatures, the application temperature of the coatings significantly influenced the magnitude of the stresses. The lowest stresses were obtained for gradient coatings for which the application temperature of the gradient coating varied continuously, proportional to the material composition in the gradient coating. 


\section{ACKNOWLEDGEMENTS}

I would like to express my sincere appreciation and gratitude to my advisor Professor M. W. Hyer for his constant guidance, support, and encouragement. I would also like to thank Professors M. S. Cramer, R. A. Heller, R. W. Hendricks, and K. L. Reifsnider for their willingness and time to serve on my committee and to review this dissertation. Thanks are also due to Professor R. K. Kapania, my advisor for my Master's degree, under whose guidance I learned the essentials to succeed in my $\mathrm{Ph}$.D. research. This work was supported by grant NAG-1-343 from the NASA Langley Research Center. The grant monitor was Dr. Wallace L. Vaughn. The financial support of the grant is sincerely appreciated.

Special thanks to my wife Petra for her love, support, and understanding. This work is dedicated to my late grandfather, Otto Wunderlich. 


\section{Contents}

1 Introduction $\quad 1$

1.1 High Temperature Materials . . . . . . . . . . . . . . 2

1.2 Applications of Carbon/Carbon Composites . . . . . . . . . . 3

1.3 Carbon/Carbon Composites . . . . . . . . . . . . . 6

1.3.1 Processing and Properties ................... 6

1.3.2 Oxidation and Oxidation Protection ............ 8

1.4 Analysis of Carbon/Carbon Composites . . . . . . . . . . . 11

1.4.1 Thermomechanical Modeling of Carbon/Carbon Composites and Coat-

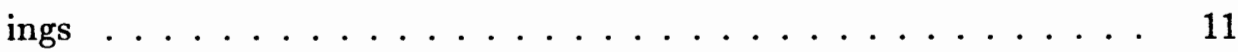

1.4.2 Objectives of the Present Research _. . . . . . . . . 14

1.5 Overview of the Dissertation $\ldots \ldots \ldots \ldots \ldots$

2 Incremental Temperature-Dependent Analysis 19

2.1 Incremental Constitutive Equations . . . . . . . . . . . . . . . 20

2.1.1 Incremental Constitutive Equations Based on Functional Form of Mechanical Strain . . . . . . . . . . . . . . . 21

2.1.2 Incremental Constitutive Equations Based on Difference in Stress

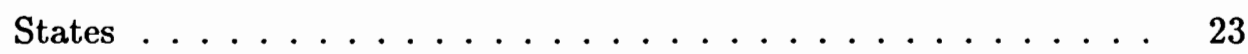

2.2 Incremental Finite Element Model . . . . . . . . . . . . 25

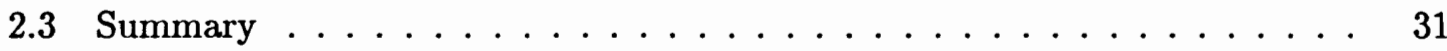


3 Simplified Analytical Solutions $\quad 32$

3.1 Incremental Simplified Plane-Stress Analysis . . . . . . . . . . . . 32

3.2 Incremental Analysis of an Isotropic Axisymmetric Infinitely Long Cylinder 35

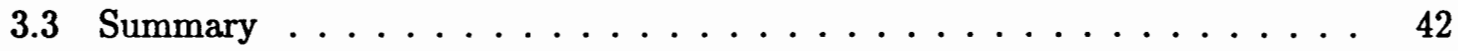

4 Verification of the Solutions

4.1 Verification of the Incremental Simplified Plane-Stress Analysis . . . . . . 43

4.2 Test Cases to Verify the Incremental Axisymmetric Elasticity Solution . . . 46

4.3 Verification of the Finite Element Model . . . . . . . . . . . . . 47

4.3.1 Comparison to ABAQUS .................... 48

4.3.2 Comparison to Axisymmetric Elasticity Solutions . . . . . . . . . . 48

4.3.2.1 Comparison to the Incremental Axisymmetric Elasticity Solution ........................ 48

4.3.2.2 Comparison to an Axisymmetric Elasticity Solution with a Gradient Layer . . . . . . . . . . . . . . . . 52

4.3.3 Comparison to the Incremental Simplified Plane-Stress Analysis _. 52

4.3.4 Comparison of Finite Element Analyses . . . . . . . . . . . . 54

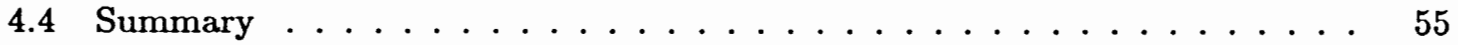

5 Temperature-Independent Results $\quad 58$

5.1 Representation of the Results . . . . . . . . . . . . . . . 59

5.2 Convergence Studies . . . . . . . . . . . . . . . . 60

5.3 Influence of Corner Region on Stresses . . . . . . . . . . . . 67

5.4 Influence of Substrate Orthotropy . . . . . . . . . . . . 78

5.5 Influence of the Corner Radius $\ldots \ldots \ldots \ldots$

5.6 Summary . . . . . . . . . . . . . . . . . . 88

6 Temperature-Dependent Results $\quad 89$

6.1 The Baseline Cases . . . . . . . . . . . . . . . . . 90 
6.1.1 Incremental Plane-Stress Analyses of Baseline Cases . . . . . . .

6.2 Comparison of Temperature-Dependent and Temperature-Independent Analyses of Baseline Cases . . . . . . . . . . . . . . . . . . 92

6.3 Temperature-Dependent Stresses in a Carbon-Carbon Substrate with a $\mathrm{SiC}$

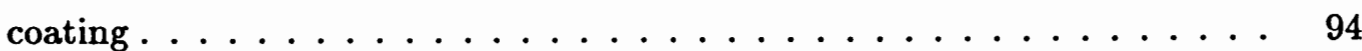

6.4 Compensating/Compliant Coating Concept for Stress Reduction in Coatings 104

6.4.1 Influence of Thickness of a Pyrolytic Carbon Coating on Stresses . . 104

6.4.2 Influence of the Coefficient of Thermal Expansion of the Compliant Coating on Stresses . . . . . . . . . . . . 111

6.4.3 Influence of the Modulus of Elasticity of the Compliant Coating on Stresses in the Coatings . . . . . . . . . . . . . . 120

6.4.4 Influence of Orthotropic Compliant Coatings . . . . . . . . 127

6.4.4.1 Orthotropic Variation of the Coefficient of Thermal Expan$\operatorname{sion} \ldots \ldots \ldots \ldots \ldots \ldots \ldots \ldots$

6.4.4.2 Orthotropic Variation of the Modulus of Elasticity . . . . . 132

6.4.5 Summary of Material Effects on Stresses in Coatings and Substrate 141

6.5 Gradient Coating Concept for Stress Reduction . . . . . . . . . . . . . . 152

6.5.1 Linear Pyrolytic Carbon to SiC Gradient Coating . . . . . . . . . . 152

6.5.2 Variations of the Gradient Coating Profiles . . . . . . . . . 156

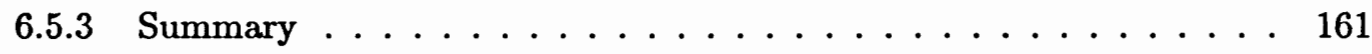

6.6 Multiple Coatings Applied at Different Temperatures _ . . . . . . . . . 162

6.6 .1 Discrete Coatings $\ldots \ldots \ldots \ldots \ldots$. . . . . . . . . . 162

6.6.2 Gradient Coatings Applied at Multiple Temperatures . . . . . . 170

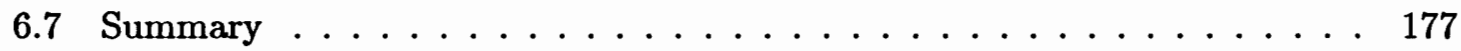

7 Summary and Future Work $\quad 184$

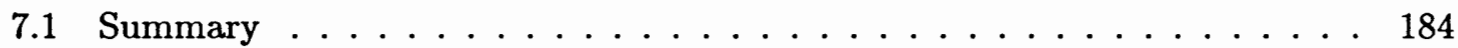

7.2 Future Work . . . . . . . . . . . . . . . 187 
A Temperature-Dependent Material Properties $\quad 194$

B Comparison of Incremental Constitutive Equations $\quad 198$

C Material Stiffness Matrix and its Transformation 204

D Implementation of the Finite Element Model 208

D.1 Mesh Generation . . . . . . . . . . . . . . . . . . 208

D.2 Material Data Input . . . . . . . . . . . . . . 209

D.2.1 Evaluation of Temperature-Dependent Material Properties _ . . . 209

D.2.2 Evaluation of Gradient Layer Properties . . . . . . . . . . . . . 210

D.3 Numerical Intergration of Shape Functions of Finite Elements . . . . . . . 210

D.3.1 Properties at Gauss Points in a Gradient Coating . . . . . . . . . . . 212

D.3.2 Transformation Angle in Corner Region . . . . . . . . . . . . 214

D.4 Solution Procedure for the Finite Element Program . . . . . . . . . . 215

D.5 Special Case: Temperature-Independent Analysis . . . . . . . . . . . 216 


\section{List of Figures}

1.1 Strength/temperature regimes of some high temperature materials. . . . . 4

1.2 Temperature distribution on the space shuttle orbiter during re-entry. . . . 5

1.3 Geometry of $\mathrm{C} / \mathrm{C}$ substrate with several coatings. . . . . . . . . . . 16

2.1 A typical problem for the analysis of a $\mathrm{C} / \mathrm{C}$ substrate with coatings. . . . 21

2.2 Coordinate system for the generalized plane-strain theory. . . . . . . . . 28

4.1 Convergence of stress calculated using the ISPSA with an increasing number of progressively different constant-property layers to stress calculated using the ISPSA with a single gradient layer. . . . . . . . . . . . 45

4.2 Finite element mesh for the quarter cylinder. . . . . . . . . . . . 49

4.3 Geometry of the quarter cylinder model . . . . . . . . . . . . . . 49

4.4 Verification of gradient layer option for finite element program. . . . . . . 51

4.5 Comparison of finite element results and elasticity solution for a gradieint

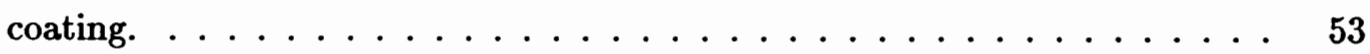

4.6 Convergence of the axial stress $\sigma_{x}$ calculated using the finite element model to the stress calculated using the ISPSA when moving away from the corner. 54

4.7 Stress distributions through the thickness of the coating and part of the substrate at different locations in the substrate. . . . . . . . . 56

5.1 Modeled region of a $\mathrm{C} / \mathrm{C}$ substrate with two coatings and coordinate system

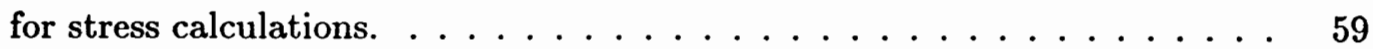


5.2 Finite element meshes used in the convergence study. . . . . . . . .

5.3 Convergence of tangential stress $\sigma_{s}$ in $\mathrm{Si}_{3} \mathrm{~N}_{4}$ coating for a substrate with two $0.05 \mathrm{~mm}$ thick coatings.

5.4 Convergence of axial stress $\sigma_{z}$ in $\mathrm{Si}_{3} \mathrm{~N}_{4}$ coating for a substrate with two $0.05 \mathrm{~mm}$ thick coatings.

5.5 Convergence of normal stress $\sigma_{n}$ in $\mathrm{Si}_{3} \mathrm{~N}_{4}$ coating for a substrate with two $0.05 \mathrm{~mm}$ thick coatings.

5.6 Convergence of shear stress $\tau_{s n}$ in $\mathrm{Si}_{3} \mathrm{~N}_{4}$ coating for a substrate with two $0.05 \mathrm{~mm}$ thick coatings.

5.7 Convergence of normal stress $\sigma_{n}$ in $\mathrm{Si}_{3} \mathrm{~N}_{4}$ coating for a substrate with two $0.1 \mathrm{~mm}$ thick coatings.

5.8 Convergence of normal stress $\sigma_{n}$ in $\mathrm{Si}_{3} \mathrm{~N}_{4}$ coating for a substrate with two $0.2 m m$ thick coatings. . . . . . . . . . . . . 66

5.9 Models considered in the parametric study. . . . . . . . . . . . 68

5.10 Effect of substrate width on stress distribution in $\mathrm{B}_{4} \mathrm{C}$ coating. . . . . . . 70

5.11 Effect of substrate width on stress distribution in $\mathrm{Si}_{3} \mathrm{~N}_{4}$ coating. . . . . . 71

5.12 Effect of modulus of the inner coating on stress distribution in inner coating. 73

5.13 Effect of modulus of the inner coating on stress distribution in $\mathrm{Si}_{3} \mathrm{~N}_{4}$ coating. 74

5.14 Details of normal stress $\sigma_{n}$ in inner coating for different moduli of the inner

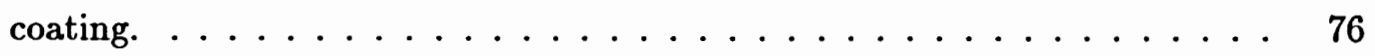

5.15 Details of shear stress $\tau_{s n}$ in inner coating for different moduli of the inner

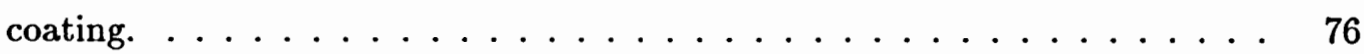

5.16 Details of normal stress $\sigma_{n}$ in $\mathrm{Si}_{3} \mathrm{~N}_{4}$ coating for different moduli of the inner

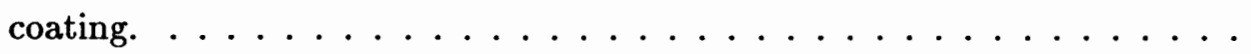

5.17 Details of shear stress $r_{s n}$ in $\mathrm{Si}_{3} \mathrm{~N}_{4}$ coating for different moduli of the inner

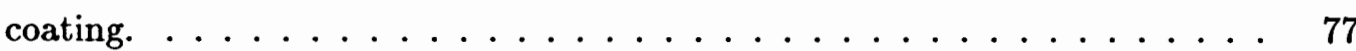

5.18 Effect of substrate orthotropy on stress distribution in the $\mathrm{B}_{4} \mathrm{C}$ coating. . . 80

5.19 Effect of substrate corner radius on stress distribution in the $\mathrm{Si}_{3} \mathrm{~N}_{4}$ coating. 83 
5.20 Effect of substrate corner radius on stress distribution in the $\mathrm{B}_{4} \mathrm{C}$ coating. . 84

5.21 Details of normal stress $\sigma_{n}$ in $\mathrm{Si}_{3} \mathrm{~N}_{4}$ coating for different substrate corner radii. 85

5.22 Details of shear stress $\tau_{s n}$ in $\mathrm{Si}_{3} \mathrm{~N}_{4}$ coating for different substrate corner radii. 85

5.23 Details of normal stress $\sigma_{n}$ in $\mathrm{B}_{4} \mathrm{C}$ coating for different substrate corner radii. 86

5.24 Details of shear stress $\tau_{s n}$ in $\mathrm{B}_{4} \mathrm{C}$ coating for different substrate corner radii. $\quad 86$

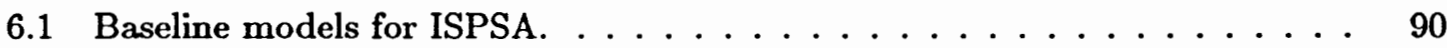

6.2 Baseline models for cases including a corner. . . . . . . . . . . . . . . 90

6.3 Temperature-dependent stresses for baseline cases. . . . . . . . . . . 92

6.4 Comparison of temperature-independent and temperature-dependent stresses at different temperatures for a substrate with a $\mathrm{SiC}$ coating. . . . . . . . . 94

6.5 Comparison of temperature-independent and temperature-dependent stresses at different temperatures for a substrate with a $\mathrm{Si}_{3} \mathrm{~N}_{4}$ coating and a $\mathrm{B}_{4} \mathrm{C}$

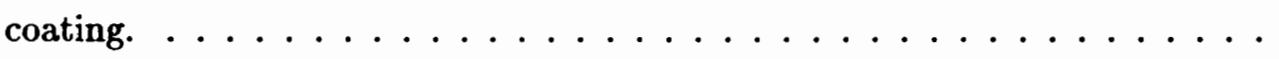

6.6 Stress distribution in the $\mathrm{SiC}$ coating at $20^{\circ} \mathrm{C}$ for a $\mathrm{C} / \mathrm{C}$ substrate with a $\mathrm{SiC}$ coating. . . . . . . . . . . . . . . . .

6.7 Maximum principal stress and maximum shear stress at $20^{\circ} \mathrm{C}$ in the $\mathrm{SiC}$ coating for a $\mathrm{C} / \mathrm{C}$ substrate with a $\mathrm{SiC}$ coating. . . . . . . . . . . 100

6.8 Stress distribution in the $\mathrm{SiC}$ coating at $500^{\circ} \mathrm{C}$ for a $\mathrm{C} / \mathrm{C}$ substrate with a $\mathrm{SiC}$ coating. . . . . . . . . . . . . . . . . . . 101

6.9 Stress distribution in the $\mathrm{SiC}$ coating at $1000^{\circ} \mathrm{C}$ for a $\mathrm{C} / \mathrm{C}$ substrate with a $\mathrm{SiC}$ coating. . . . . . . . . . . . . . . . . . . . 102

6.10 Stress distribution in the $\mathrm{SiC}$ coating at $1500^{\circ} \mathrm{C}$ for a $\mathrm{C} / \mathrm{C}$ substrate with a $\mathrm{SiC}$ coating. . . . . . . . . . . . . . . . . . . . . 103

6.11 Tangential and normal stresses in $\mathrm{SiC}$ coating for different thicknesses of the pyrolytic carbon coating . . . . . . . . . . . . . . 107

6.12 Deformed and undeformed finite element meshes for the first baseline case. $\quad 110$ 
6.13 Tangential stress and normal stress in $\mathrm{SiC}$ coating for different coefficients of thermal expansion of the compliant coating. . . . . . . . . . . . . . 114

6.14 Effect of thickness and coefficient of thermal expansion of the compliant coating on the maximum stresses in the $\mathrm{SiC}$ coating. . . . . . . . . 117

6.15 Effect of thickness and coefficient of thermal expansion of the compliant coating on the maximum stresses in the compliant coating. . . . . . . 118

6.16 Effect of thickness and coefficient of thermal expansion of the compliant coating on the maximum stresses in the $\mathrm{C} / \mathrm{C}$ substrate. . . . . . . . . . 119

6.17 Effect of thickness and modulus of elasticity of the compliant coating on the maximum stresses in the $\mathrm{SiC}$ coating. . . . . . . . . . . . .

6.18 Effect of thickness and modulus of elasticity of the compliant coating on the maximum stresses in the compliant coating. . . . . . . . . . . . . . 123

6.19 Effect of thickness and modulus of elasticity of the compliant coating on the maximum stresses in the $\mathrm{C} / \mathrm{C}$ substrate. . . . . . . . . . . . .

6.20 Effect of coefficient of thermal expansion and modulus of the compliant coating on stresses in the $\mathrm{SiC}$ coating and the compliant coating. Comparison of maximum tangential stresses and maximum normal stresses. . . . . . . . .

6.21 Effect of coefficient of thermal expansion and modulus of the compliant coating on stresses in the $\mathrm{SiC}$ coating and the compliant coating. Comparison of maximum axial stresses and maximum shear stresses. . . . . . . . . . .

6.22 Effect of thickness and transverse coefficient of thermal expansion of the compliant coating on the maximum stresses in the $\mathrm{SiC}$ coating. . . . . . .

6.23 Effect of thickness and transverse coefficient of thermal expansion of the compliant coating on the maximum stresses in the compliant coating. . . .

6.24 Effect of thickness and transverse coefficient of thermal expansion of the compliant coating on the maximum stresses in the $\mathrm{C} / \mathrm{C}$ substrate. . . . . .

6.25 Effect of thickness and inplane coefficients of thermal expansion of the compliant coating on the maximum stresses in the $\mathrm{SiC}$ coating. . . . . . . . 136 
6.26 Effect of thickness and inplane coefficients of thermal expansion of the compliant coating on the maximum stresses in the compliant coating. . . . . . 137

6.27 Effect thickness and inplane coefficients of thermal expansion of the compliant coating on the maximum stresses in the $\mathrm{C} / \mathrm{C}$ substrate. . . . . . . . .

6.28 Effect of thickness and quasi-isotropic moduli of the compliant coating on the maximum stresses in the $\mathrm{SiC}$ coating. . . . . . . . . . . . . 142

6.29 Effect of thickness and quasi-isotropic moduli of the compliant coating on the maximum stresses in the compliant coating. . . . . . . . . . .

6.30 Effect of thickness and quasi-isotropic moduli of the compliant coating on the maximum stresses in the $\mathrm{C} / \mathrm{C}$ substrate. . . . . . . . . . . . . 144

6.31 Effect of thickness and transverse modulus of the compliant coating on the maximum stresses in the $\mathrm{SiC}$ coating. . . . . . . . . . . .

6.32 Effect of thickness and transverse modulus of the compliant coating on the maximum stresses in the compliant coating. . . . . . . . . . . . . 146

6.33 Effect of thickness and transverse modulus of the compliant coating on the maximum stresses in the $\mathrm{C} / \mathrm{C}$ substrate. . . . . . . . . . . .

6.34 Effect of thickness and inplane moduli of the compliant coating on the maximum stresses in the $\mathrm{SiC}$ coating. . . . . . . . . . . . . . 148

6.35 Effect of thickness and inplane moduli of the compliant coating on the maximum stresses in the compliant coating. . . . . . . . . . . . .

6.36 Effect of thickness and inplane moduli of the compliant coating on the maximum stresses in the $\mathrm{C} / \mathrm{C}$ substrate. . . . . . . . . . . . . 150

6.37 Geometry of a C/C substrate with a gradient coating and a SiC coating. . . 152

6.38 Tangential stress in SiC coating near interface for different gradient coating thicknesses. . . . . . . . . . . . . . . . 155

6.39 Variation of properties through a gradient coating. . . . . . . . . . 158

6.40 Tangential stress $\bar{\sigma}_{s}$ near the SiC-gradient coating in the SiC coating for different property distributions in the gradient coating. . . . . . . . . . 160 
6.41 Normalized thickness locations for stress calculations.

6.42 Stress distribution in the $\mathrm{Si}_{3} \mathrm{~N}_{4}$ coating at $20^{\circ} \mathrm{C}$ for a $\mathrm{C} / \mathrm{C}$ substrate with a $\mathrm{B}_{4} \mathrm{C}$ coating applied at $1000^{\circ} \mathrm{C}$ and $\mathrm{a} \mathrm{Si}_{3} \mathrm{~N}_{4}$ coating applied at $1500^{\circ} \mathrm{C} . \quad .165$

6.43 Stress distribution in the $\mathrm{B}_{4} \mathrm{C}$ coating at $20^{\circ} \mathrm{C}$ for a $\mathrm{C} / \mathrm{C}$ substrate with a $\mathrm{B}_{4} \mathrm{C}$ coating applied at $1000^{\circ} \mathrm{C}$ and $\mathrm{a} \mathrm{Si}_{3} \mathrm{~N}_{4}$ coating applied at $1500^{\circ} \mathrm{C} . \quad$. 166

6.44 Stress distribution in the $\mathrm{C} / \mathrm{C}$ substrate at $20^{\circ} \mathrm{C}$ for a $\mathrm{C} / \mathrm{C}$ substrate with a $\mathrm{B}_{4} \mathrm{C}$ coating applied at $1000^{\circ} \mathrm{C}$ and $\mathrm{aSi}_{3} \mathrm{~N}_{4}$ coating applied at $1500^{\circ} \mathrm{C} . \quad \ldots 167$

6.45 Geometry of coatings for multiple gradient coatings. . . . . . . . . . . . 171

6.46 Locations for through-thickness plots. . . . . . . . . . . . . . 174

6.47 Through-the-thickness variation of stresses in the far field, location 1. . . 178

6.48 Through-the-thickness variation of stresses at the beginning of the corner,

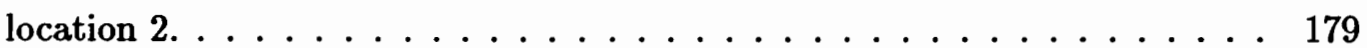

6.49 Through-the-thickness variation of stresses at the center of the corner, location $3 . \ldots \ldots \ldots \ldots \ldots \ldots \ldots$

6.50 Through-the-thickness variation of stresses at the end of the corner, location

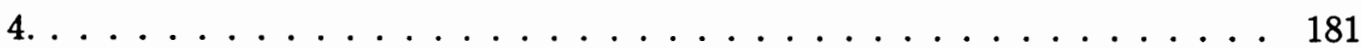

6.51 Through-the-thickness variation of stresses at the horizontal line of symmetry,

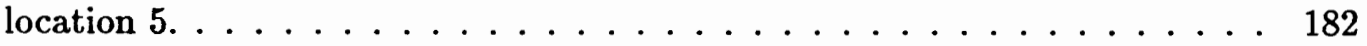

A.1 Temperature-dependent properties for a C/C substrate. . . . . . . . . 197

B.1 Clamped-clamped beam. . . . . . . . . . . . . . . . . 198

C.1 Rotation from a $x-y-z$ global coordinate system to the 1-2-3 material coordinate system. . . . . . . . . . . . . . . . 205

D.1 Example of a mesh generated by the preprocessor. . . . . . . . . 209

D.2 Available element geometries. . . . . . . . . . . . . . 211

D.3 Parameters used for special numerical integration. . . . . . . . . . . 213

D.4 Calculation of properties in a gradient coating. . . . . . . . . . . 214 
D.5 Calculation of transformation angle. . . . . . . . . . . . 215 


\section{List of Tables}

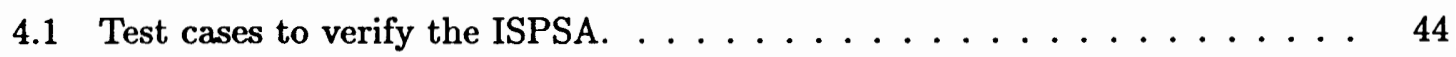

4.2 Test cases to verify the incremental axisymmetric elasticity solution. . . . 4 46

4.3 Test cases for comparison of finite element results to the axisymmetric elasticity solution. $\ldots \ldots \ldots \ldots \ldots \ldots \ldots \ldots \ldots \ldots \ldots \ldots \ldots \ldots$

5.1 Number of elements and number of nodes in the finite element meshes used

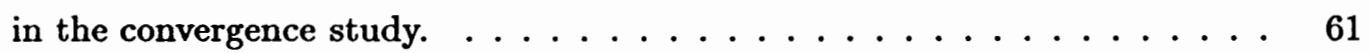

5.2 Comparison of far-field stresses calculated using the finite element model for different width models and stresses calculated using ISPSA . . . . . . . 72

5.3 Beginning and ending $s$-coordinate for the corner region for different corner

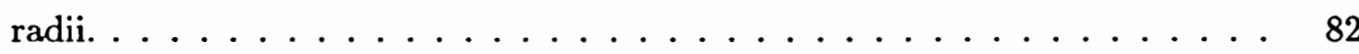

6.1 Temperature-dependent stresses from ISPSA for baseline cases. . . . . . . 92

6.2 Maximum stresses for different pyrolytic carbon to $\mathrm{SiC}$ thickness ratios. . . 106

6.3 Maximum stresses for different coefficients of thermal expansion of the compliant coating for $t_{\text {compl. }} / t_{\text {total }}=1 / 3 \ldots \ldots \ldots \ldots \ldots \ldots \ldots$

6.4 Maximum stresses for different pyrolytic carbon-to-SiC gradient coating thick-

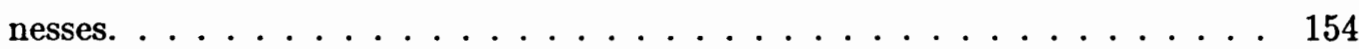

6.5 Maximum strains for different pyrolytic carbon-to-SiC gradient coating thicknesses. . . . . . . . . . . . . . . . . . . . . . 157 
6.6 Maximum stresses for different material property distributions through-thethickness of the gradient coating. . . . . . . . . . . . . . . . 159

6.7 Effect of thickness of convex gradient coating on stresses in the $\mathrm{SiC}$ coating. 160

6.8 Effect of gradient distribution on stresses in the coating and the substrate for thick gradient coatings. . . . . . . . . . . . . . . . 161

6.9 Comparison of far field stresses calculated using the ISPSA and the finite element model (FEM) for multiple coatings. . . . . . . . . . . . . . . . 164

6.10 Maximum tangential stresses $\bar{\sigma}_{s}$ for multiple coatings on a C/C substrate . 172

6.11 Normalized stresses in a $\mathrm{B}_{4} \mathrm{C}$ coating for different coating application temperatures.

A.1 Application temperatures for coatings. . . . . . . . . . . . . 195

A.2 Temperature-dependent moduli of elasticity and Poisson ratios for C/C substrate. . . . . . . . . . . . . . . . . 195

A.3 Temperature-dependent coefficient of thermal expansion for C/C substrate. 196

A.4 Temperature-dependent properties for SiC . . . . . . . . . . . . . 196

A.5 Temperature-dependent properties for $\mathrm{B}_{4}$ C $\ldots \ldots \ldots \ldots$

A.6 Temperature-dependent properties for $\mathrm{Si}_{3} \mathrm{~N}_{4} \ldots \ldots \ldots \ldots \ldots$

A.7 Properties for pyrolytic carbon. . . . . . . . . . . . . . . . . 196

B.1 Comparison of the stresses calculated using the exact solution and the incremental constitutive equations based on the difference in stress states at two different temperatures. . . . . . . . . . . . . . . . . . . 200

B.2 Stresses calculated using the incremental constitutive equations based on an assumed functional form for 10 and 20 temperature increments. . . . . . . . 201

B.3 Stresses calculated using the incremental constitutive equations based on an assumed functional form for 100 and 1000 temperature increments. . . . . 201

B.4 Effect of the coupling term on the stresses. . . . . . . . . . . 202 


\section{Chapter 1}

\section{Introduction}

The research presented in this dissertation focused on the prediction of stresses in coatings on carbon-carbon composites. Carbon-carbon composites are candidate materials for high temperature applications. However, because they tend to oxidize at elevated temperatures, coatings are applied to carbon-carbon composites for oxidation protection. Due to the thermal expansion mismatch between the carbon-carbon substrate and the coatings, the coatings can microcrack, which then leads to renewed oxidation of the carbon-carbon substrate. To be able to design coatings that do not microcrack, the stresses generated in the coatings during processing and service conditions of the carbon-carbon composite have to be understood. Thus, the aim of the present research was to shed light on the nature of stresses in coatings on carbon-carbon composites, and to provide the material designer with a tool to study concepts that might lead to reduced stresses in the coatings and result in crack-free coatings. Additionally, several coating and material architecture concepts were explored to provide insight into possible reduction of coating stresses.

In the following, a review of carbon-carbon composites is given. First, in Section 1.1 a motivation for the use of high temperature materials is given and it is described where carbon-carbon composites can be used. Then in Section 1.2 some applications for which carbon-carbon composites are already successfully used are described. Next the processing 
and properties of carbon-carbon composites and concepts for the oxidation protection are discussed in Sections 1.3.1 and 1.3.2, respectively. Literature relevant to the thermomechanical analysis of carbon-carbon composites, coatings, and functional gradient materials is reviewed in Section 1.4. Then the objectives of the present research and the approach taken are described. Finally, in Section 1.5 a detailed outline of the chapters to follow is given.

\subsection{High Temperature Materials}

A good motivation for the use of high temperature materials is given by Meetham [49]. Meetham cites several examples in which the use of high temperature materials results in more efficient products. More efficient products are especially important in industries for which the price of fuel is a major concern. For example, the efficiency of gas turbine engines can be increased by $6 \%$ if the operating temperature is increased by $150^{\circ} \mathrm{C}$. Other applications in which high temperature materials are essential are space vehicles, high speed aircraft, and rocket engine cases. All of these applications involve materials with properties that are not exhibited by conventional materials. The objective of ongoing research is to develop material systems for the use in the temperature range between $500^{\circ} \mathrm{C}$ and $2000^{\circ} \mathrm{C}[6,63]$. High temperature material systems include high specific strength and high temperature intermetallic compounds, as well as fiber reinforced ceramics. The high specific strength intermetallic compounds under development are TiAl alloys. These alloys are expected to reach a strength of over $100 \mathrm{MPa}$ at temperatures over $1100^{\circ} \mathrm{C}$. Currently $\mathrm{TiAl}$ alloys are available for temperatures up to $800^{\circ} \mathrm{C}$, but before these can be used in aircraft structures their manufacturability and fracture behavior must be improved [6]. The high temperature intermetallic compounds are based on $\mathrm{NbAl}$ systems and are expected to reach a tensile strength of $75 \mathrm{MPa}$ at $1800^{\circ} \mathrm{C}$. Fiber reinforced ceramics like carbon/carbon $(\mathrm{C} / \mathrm{C})$, carbon/silicon carbide $(\mathrm{C} / \mathrm{SiC})$ and silicon carbide/silicon carbide $(\mathrm{SiC} / \mathrm{SiC})$ have much better fracture behavior than ceramics that are not reinforced [6]. Currently, they 
can be used for limited time at temperatures up to $1800^{\circ} \mathrm{C}$. Carbon-carbon composites with a two-directional fiber lay-up are expected to retain a tensile strength of over 700 $\mathrm{MPa}$ and a tensile modulus of over $200 \mathrm{GPa}$ after heating for $20 \mathrm{hrs}$ at $2000^{\circ} \mathrm{C}$, or after heating for $200 \mathrm{hrs}$ at $1800^{\circ} \mathrm{C}$ [63]. A comparison of the strength-temperature regimes of several high temperature materials, as shown in Fig. 1.1, indicates that while there are several materials available for temperatures up to $1500^{\circ} \mathrm{C}$, fiber reinforced ceramics are the only materials that retain their strength at higher temperatures. Among the fiber reinforced ceramics, $\mathrm{C} / \mathrm{C}$ composites have many favorable attributes, such as high damage tolerance, high toughness, excellent thermal shock resistance, fatigue resistance, and good frictional characteristics [72]. Considering those attributes, it appears that $\mathrm{C} / \mathrm{C}$ composites should be the material of choice for very high temperature applications. However, $\mathrm{C} / \mathrm{C}$ composites are not without problems. Carbon reacts rapidly with oxygen at temperatures as low as $400^{\circ} \mathrm{C}$. This reaction causes rapid degradation of the $\mathrm{C} / \mathrm{C}$ composite. Thus effective oxidation protection is necessary to successfully use $\mathrm{C} / \mathrm{C}$ composites at temperatures above $400^{\circ} \mathrm{C}$. Another drawback of $\mathrm{C} / \mathrm{C}$ composites is their very high cost. Depending on the manufacturing process, the cost may be between $\$ 600 / \mathrm{kg}$ and $\$ 6000 / \mathrm{kg}$. For many applications, the attributes of $\mathrm{C} / \mathrm{C}$ composites outweigh the limitations.

\subsection{Applications of Carbon/Carbon Composites}

Today C/C composites are successfully used in several applications. Major high temperature applications include aircraft brakes, rocket motors, heat shields for re-entry vehicles, turbine engine components, and industrial components [62,64]. Friction between rotating and stationary disks in aircraft brakes causes them to heat up to $500^{\circ} \mathrm{C}$, with surface temperatures up to $2000^{\circ} \mathrm{C}$. Technological advances that caused the price of $\mathrm{C} / \mathrm{C}$ aircraft brakes to drop from $\$ 1375 / \mathrm{kg}$ to $\$ 375 / \mathrm{kg}$ have made it commercially advantageous to use $\mathrm{C} / \mathrm{C}$ brakes in civil aircraft [64]. Probably the most well known application of $\mathrm{C} / \mathrm{C}$ composites is their use as heat shields of re-entry space vehicles. An examination of the 


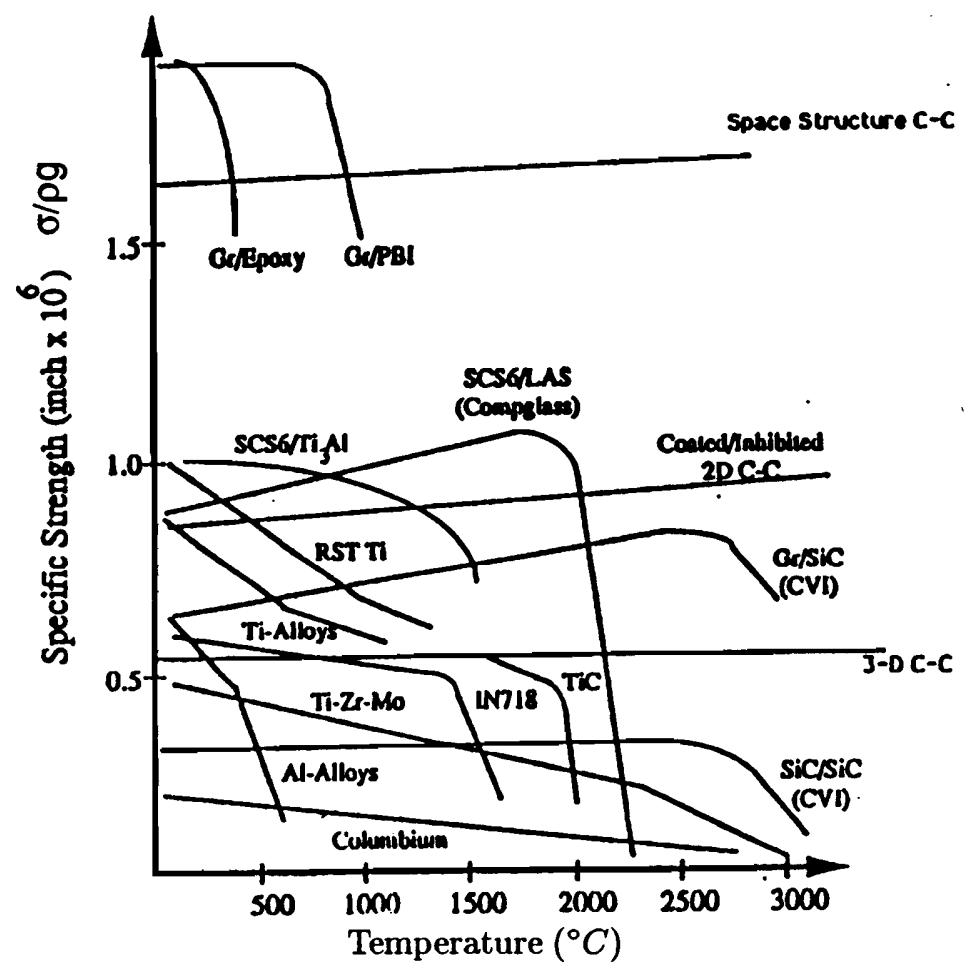

Figure 1.1: Strength/temperature regimes of some high temperature materials (from [49]).

temperature distribution during re-entry of the space shuttle orbiter, Fig. 1.2, shows that the orbiter experiences temperatures of up to $1600^{\circ} \mathrm{C}$ at the nose cone and at wing leading edges. Carbon-carbon composites with $\mathrm{SiC}$ coatings for oxidation protection are successfully used for the construction of these structural components [27]. The excellent thermal shock resistance permits rapid transition from $-160^{\circ} \mathrm{C}$ in the cold of space to the high temperatures of re-entry without fracture [64]. Industrial applications of $\mathrm{C} / \mathrm{C}$ composites include high temperature fasteners, hot press dies, and hot gas ducts. The formability of $\mathrm{C} / \mathrm{C}$ composites and thus the possibility to manufacture more complex components like integral skin-stringer panels and $\mathrm{C} / \mathrm{C}$ thermal protection panels has been demonstrated by Kinjo, et al. [36]. The different applications mentioned may require different properties of $\mathrm{C} / \mathrm{C}$ composites. In the next section it is described how the processing conditions can effect 


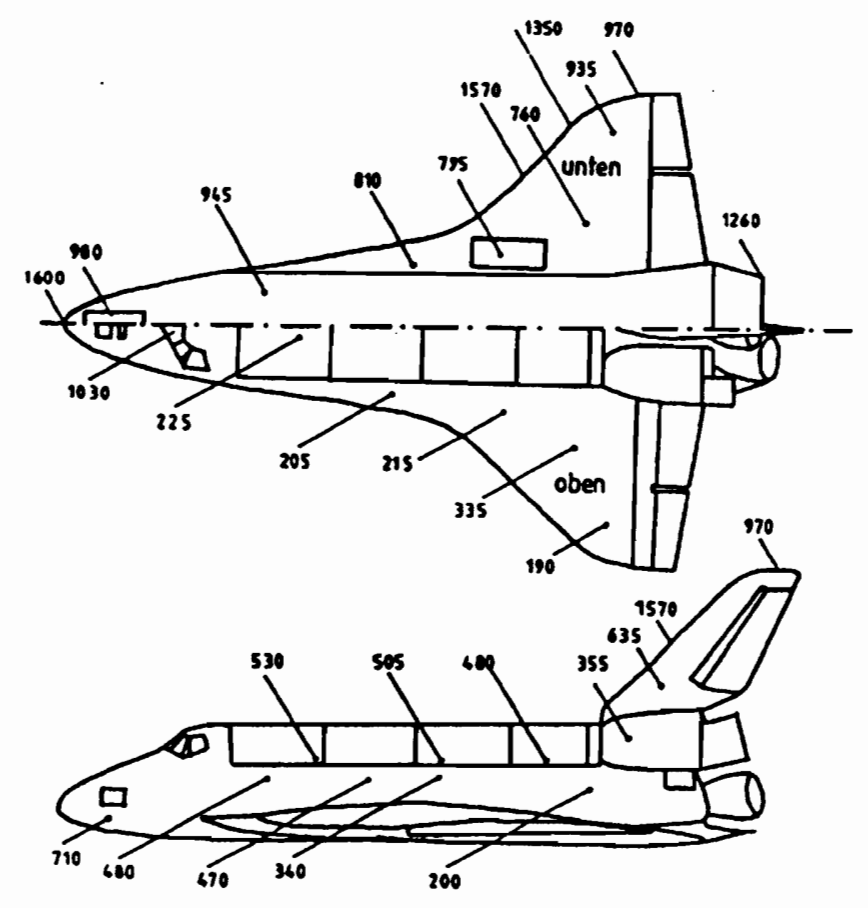

Figure 1.2: Temperature distribution $\left({ }^{\circ} \mathrm{C}\right)$ on the space shuttle orbiter during re-entry (from [27]).

properties of $\mathrm{C} / \mathrm{C}$ composites and thus can be adjusted to tailor $\mathrm{C} / \mathrm{C}$ composite properties for specific applications.

Most of the applications mentioned in the preceding discussion require only a limited lifetime of the $\mathrm{C} / \mathrm{C}$ composites. For successful use of $\mathrm{C} / \mathrm{C}$ in applications that require a longer lifetime and greater degree of thermal cycling, like turbine engines, improvement of the oxidation resistance is required. This may possibly be achieved by using matrix inhibitors, selecting proper coating sequences, or using gradient coatings. This is a topic of current research (e.g. $[49,59,64])$. 


\subsection{Carbon/Carbon Composites}

\subsubsection{Processing and Properties}

As discussed by Savage [64], C/C composites cannot be regarded as one material system with a given set of properties. Rather, they should be regarded as a family whose properties can be tailored to specific applications. Major factors influencing the properties of $\mathrm{C} / \mathrm{C}$ composites are the carbon fiber type used, the processing method for the matrix, and the architecture of the carbon fiber preform. Ngai [52] gives a good summary of the processing and material characteristics of $\mathrm{C} / \mathrm{C}$ composites.

A good classification of available carbon fibers and their processing parameters and the resulting properties is given by Fitzer [20]. Another more detailed review of the processing of carbon fibers and the influence of the processing parameters on their properties is given by Savage [64]. The properties of carbon fibers depend mainly on the precursor used to manufacture the fibers, and on the heat treatment of the fibers. The precursors currently used are rayon, polyacrylonitrile (PAN), and mesophase pitch. The most important steps in the processing of the fibers are the carbonization and graphitization. During carbonization the carbon content of the material is increased from the precursor, usually by pyrolysis, until an almost pure carbon residue is obtained. During the graphitization process the structure of the carbon residue is transformed into a graphitic structure. The degree of graphitization depends on the heat treatment temperature. Heat treatment is classified into low (up to $1400^{\circ} \mathrm{C}$ ), intermediate $\left(1300^{\circ} \mathrm{C}\right.$ to $2000^{\circ} \mathrm{C}$ ), and high (above $2000^{\circ} \mathrm{C}$ ) temperature treatment [20]. In general, the modulus of the carbon fibers increases with heat treatment temperature, while the strength reaches a maximum at intermediate heat treatment temperatures. PAN based fibers generally have a higher strength and a lower modulus than pitch based fibers. Properties of available PAN based fibers range from fibers with a tensile modulus of $300 \mathrm{GPa}$ and a tensile strength of $7000 \mathrm{MPa}$, to fibers with a tensile modulus of $500 \mathrm{GPa}$ and a tensile strength of $4000 \mathrm{MPa}$. The range of pitch based fibers is from fibers with a tensile modulus of $500 \mathrm{GPa}$ and a tensile strength of $3000 \mathrm{MPa}$, to fibers with 
a tensile modulus of $740 \mathrm{GPa}$ and a strength of $2800 \mathrm{MPa}$. Rayon based fibers generally have a lower strength than PAN or pitch based fibers [64].

Processing of the matrix for $\mathrm{C} / \mathrm{C}$ is usually achieved by chemical vapor deposition (CVD) or by multiple impregnation pyrolysis using thermosets or thermoplastics. Chemical vapor deposition yields the best carbon matrices and thus the best composite properties. However, the process is extremely slow and, therefore, very expensive. Also, the processing parameters in CVD have a large influence on the final properties, and hence the process has to be monitored carefully. The least expensive of the processes is multiple impregnation pyrolysis using thermosets. The quality of the composite obtained using this process, however, is not as good.

Goetzel [23] examined the variation of properties of $\mathrm{C} / \mathrm{C}$ composites with temperature. Two composites made of rayon precursor carbon fiber square-weave cloth with different densities, and one composite made from a square-weave fabric of fibers derived from PAN, were tested. The nominal densities of these three composites were $1525 \mathrm{~kg} / \mathrm{m}^{3}, 1650 \mathrm{~kg} / \mathrm{m}^{3}$, and $1850 \mathrm{~kg} / \mathrm{m}^{3}$, respectively. Goetzel found that the higher density composites had the better properties, but that the overall variation of properties with temperature for all three composites was similar. Goetzel observed that for all materials the tensile strength, the compressive strength, and also the shear strength and the interlaminar strength increased with temperature. Huettner, et al. [31] attribute the increasing strength to the $\mathrm{C} / \mathrm{C}$ fibermatrix interface interactions. The carbon fibers expand transversely more than the carbon matrix and therefore a more effective load transfer is possible at higher temperatures. The moduli of the materials in Goetzel's study remained constant for temperatures up to $2275^{\circ} \mathrm{C}$. Above $2275^{\circ} \mathrm{C}$ all moduli except for the tensile modulus of the PAN based composite began to drop, a characteristic which Goetzel attributed to the onset of plastic deformation. The coefficient of thermal expansion of all composites increased with temperature. For the rayon based composites the inplane coefficient of thermal expansion varied between $1.0 \times 10^{-6} /{ }^{\circ} \mathrm{C}$ at $775^{\circ} \mathrm{C}$ and $3-4 \times 10^{-6} /{ }^{\circ} \mathrm{C}$ at $3275^{\circ} \mathrm{C}$, while the transverse coefficient of thermal expansion of the PAN based composite varied between $3-4 \times 10^{-6} /{ }^{\circ} \mathrm{C}$ at $775^{\circ} \mathrm{C}$ and $8 \times 10^{-6} /{ }^{\circ} \mathrm{C}$ at 
$3275^{\circ} \mathrm{C}$. The inplane coefficient of thermal expansion of the PAN based composite was negative at low temperatures and increased to $2 \times 10^{-6} /{ }^{\circ} \mathrm{C}$ at temperatures above $2275^{\circ} \mathrm{C}$. The transverse coefficient of thermal expansion varied between 10 and $13 \times 10^{-6} /{ }^{\circ} \mathrm{C}$ over the temperature range. Properties of $\mathrm{C} / \mathrm{C}$ composites with other preform constructions, e.g., three-dimensional Cartesian and polar weaves, can be found in [52]. The specific properties of the $\mathrm{C} / \mathrm{C}$ composite used in the present study are given in Appendix A.

\subsubsection{Oxidation and Oxidation Protection}

As mentioned at the onset, the rapid reaction of carbon with oxygen at temperatures as low as $400^{\circ} \mathrm{C}$ is the limiting factor in the use of $\mathrm{C} / \mathrm{C}$ composites. Usually external coatings are applied to the $\mathrm{C} / \mathrm{C}$ composite to avoid oxidation. These coatings are deposited on the $\mathrm{C} / \mathrm{C}$ composite at temperatures between $1000^{\circ} \mathrm{C}$ and $1800^{\circ} \mathrm{C}$. Because of the large mismatch in coefficient of thermal expansion between the coatings and the $\mathrm{C} / \mathrm{C}$ composite, the coatings microcrack when the composite is cooled. Oxygen can then be transported through the microcracks and cause oxidation of the substrate. Extensive research efforts are underway to try to improve the oxidation protection of $\mathrm{C} / \mathrm{C}$ composites (e.g. $[10,17,28])$.

The onset of oxidation can be raised above $650^{\circ} \mathrm{C}$ by the use of substrate inhibitors. These inhibitors are ceramics, usually boron or boron compounds, that are placed within the carbon matrix and that form glasses which flow within the structure and provide oxygen barriers [59,64]. Gee and Little [22] tested a combination of a $\mathrm{SiC}$ coating with a $\mathrm{B}_{2} \mathrm{O}_{3}$ inhibitor. They found that this combination protects $\mathrm{C} / \mathrm{C}$ composites at temperatures up to $1000^{\circ} \mathrm{C}$ for extended periods of time. The fluidity of the glass sealed the microcracks at temperatures above $500^{\circ} \mathrm{C}$ and thus prevented oxidation of the substrate. The use of borate glasses as crack sealants is restricted to temperatures below $1500^{\circ} \mathrm{C}$ because above this temperature the glasses become volatile. Short-term protection of the $\mathrm{C} / \mathrm{C}$ substrate at temperatures between $1500^{\circ} \mathrm{C}$ and $1750^{\circ} \mathrm{C}$ can be achieved by using $\mathrm{SiC}$ and $\mathrm{Si}_{3} \mathrm{~N}_{4}$ outer coatings and no inhibitors. Above this temperature multilayer coating systems have to be used [64-66]. Multilayer coating systems are necessary to provide protection 
against oxidation and erosion, while maintaining chemical compatibility. A coating sequence suggested by Sheehan [65] consists of a refractory oxide outer layer as an erosion barrier, a $\mathrm{SiO}_{2}$ glass as an oxygen barrier, and a refractory oxide and a refractory carbide for chemical compatibility. Each material in such a multilayer coating has its own unique set of material properties, failure characteristics, and coating application temperature. Other research efforts to eliminate or reduce the microcracking of the coatings include the development of $\mathrm{C} / \mathrm{C}$ composites with a higher coefficient of thermal expansion or the use of very low coefficient of thermal expansion coating materials [43]. Kinjo, et al. [36] and Strife [69] have shown that the application of coatings to $\mathrm{C} / \mathrm{C}$ substrates does not degrade the mechanical properties of the composite.

More recently the use of functional gradient coatings to reduce the stresses in the coatings and prevent microcracking was suggested. As stated by Cherradi, et al. [14]:

Functional gradient materials (FGMs) are a new class of advanced materials characterized by compositional and/or microstructural gradation over macroscopic/microscopic distances.

Cherradi, et al. give a summary of recent developments in FGMs. Duva, et al. [19] showed that FGMs can be used to significantly lower stresses in engineering problems. They found that the loading required to initiate plastic deformation at stress concentrations in an infinite plate with a circular hole and in a matrix that is perfectly bonded to an infinitely long, rigid circular cylindrical fiber could be doubled through the proper design of the material. Different methods for processing of FGMs are described by Shiota, et al. [67] and by Cherradi, et al. [14]. Lannutti [41] mentions several issues that can be controlled by the composite architecture of FGMs. These include strength, toughness, thermal shock resistance, and creep. Functional gradient materials are used as functional gradient coatings, functional gradient joints, and functional gradient materials themselves (Cherradi, et al. [14]). The following discussion focuses on functional gradient coatings.

Functional gradient coatings were first used as thermal barrier coatings [37]. The idea 
of using FGMs as a thermal barrier material was to prepare a new composite by using heat resistant ceramics on the high temperature surface and tough metals on the low temperature surface, with gradual composite variation from ceramic to metal. FGMs have also been used as fiber coatings to reduce stresses between fibers and matrices [32,60].

Of interest for the present research is the use of FGMs as coatings on $\mathrm{C} / \mathrm{C}$ composites. Sohda, et al. [68] experimentally studied microcracking of $\mathrm{SiC} / \mathrm{C}$ functional gradient coatings on $\mathrm{C} / \mathrm{C}$ composites. They used CVD to deposit SiC/C FGMs on hemispherical materials of $50 \mathrm{~mm}$ diameter. By changing the CVD conditions they produced porous and dense FGMs. Thermal fatigue tests showed that the porous coating structures are resistant to cracking and delamination, while dense coatings crack and delaminate. Suemitsu, et al. [70] also studied SiC/C FGM coatings on C/C substrates. In addition, they produced modified matrix $\mathrm{C} / \mathrm{C}$ composites that had different volume fractions of $\mathrm{SiC}$ in the carbon matrix. They found that both the modified matrix composites and the SiC/C FGM coatings showed much better heat resistance than conventional $\mathrm{C} / \mathrm{C}$ composites with discrete $\mathrm{SiC}$ coatings. Kowbel, et al. $[39,40]$ investigated the oxidation protection of $\mathrm{C} / \mathrm{C}$ composites by graded $\mathrm{SiC} / \mathrm{C} \mathrm{CVD} \mathrm{coatings} \mathrm{and} \mathrm{by} \mathrm{plasma} \mathrm{assisted} \mathrm{CVD} \mathrm{silicon} \mathrm{nitride} \mathrm{coatings.} \mathrm{They}$ did not observe cracking of the coatings for either case. For the plasma assisted CVD they attributed this to the low deposition temperature of the coating and therefore to low thermal stress and fewer defects in the coating. For the graded $\mathrm{SiC} / \mathrm{C}$ coating they attributed the absence of cracks to a redistribution of the thermal stresses due to the graded interfaces. Kowbel [38] further investigated other CVD FGM coatings. These included SiC/C, $\mathrm{BN} / \mathrm{Si}_{3} \mathrm{~N}_{4}$, and $\mathrm{C} / \mathrm{B}_{4} \mathrm{C} / \mathrm{SiC}$ coatings on two-dimensional $\mathrm{C} / \mathrm{C}$ substrates. As in $[39,40]$ the gradient $\mathrm{SiC} / \mathrm{C}$ coatings produced crack free coatings. The presence of the functional gradient sealants in both the $\mathrm{BN} / \mathrm{Si}_{3} \mathrm{~N}_{4}$ and $\mathrm{C} / \mathrm{B}_{4} \mathrm{C} / \mathrm{SiC}$ coatings provided good crack filling capabilities. 


\subsection{Analysis of Carbon/Carbon Composites}

From discussions in the previous sections it is apparent that the behavior of coatings on $\mathrm{C} / \mathrm{C}$ composites is quite complex; material properties may depend on temperature, coatings may be added to the $\mathrm{C} / \mathrm{C}$ substrate at different temperatures, and coatings may have continuously varying properties through their thickness. Though several researchers experimentally studied issues related to coatings on $\mathrm{C} / \mathrm{C}$ composites ( Section 1.3.2), only few analytical or numerical studies exist which specifically address stresses in coatings on $\mathrm{C} / \mathrm{C}$ composites. Therefore in the following a more general review of literature on thermomechanical analysis of coated structures, composite materials with temperature-dependent material properties, and FGMs is given.

\subsubsection{Thermomechanical Modeling of Carbon/Carbon Composites and Coatings}

Coated structures have been analyzed by a number of researchers. However, most of them consider isotropic materials or simple geometries. Analytical models for thin coatings on isotropic substrates have been presented by Lucheta [44], Olesiak [55], Padovan [58] and Touati, et al. [73]. Padovan [58] and Touati, et al. [73] have included the effect of the growth of an oxide layer in their analysis. In a series of papers Padovan, et al. [56,57] and Taylor and Bill [71] analyzed thermal stresses in ceramic coatings on gas turbine seals using the finite element method. All of these were for the case of the coatings and the substrate being isotropic. Substantial stresses that could lead to failure of the coatings were predicted. A compensating or compliant layer concept to reduce the stresses between fibers and the matrix in metal matrix composites was studied by Arnold, et al. [7].

Copp, et al. [15] used a finite element model for the failure analysis of uncoated $\mathrm{C} / \mathrm{C}$ composites under three-point bending. Their analysis agreed well with the experimental results they obtained. Mahfuz, et al. $[45,46]$ conducted a similar analytical and experimental study of C/C composites with a SiC coating under three-point bending. In [45] they used 
a large deflection finite element model. They calculated deflections and stresses at different temperatures for given loads. A comparison of their finite element results and experiments showed that the model accurately predicted deflections, but that stresses at elevated temperatures were overestimated in coatings and underestimated in the substrate. Yamada, et al. [78] analyzed a $\mathrm{C} / \mathrm{C}$ composite with a SiC coating and a controlled compositional gradient interlayer using a plane-strain finite element model. In this study the gradient layer was approximated by several constant property layers with progressively varying material properties. They found the interlayer reduced stresses in the $\mathrm{SiC}$ coating. All the analyses in references $[15,45,46,78]$ assumed temperature-independent material properties.

Recent review papers by Noda [53] on thermal stresses in materials with temperaturedependent properties, and by Noor and Burton [54] on computational models for high temperature multilayered composite plates and shells, provide a good background on work that has been done on thermomechanical analysis. Thermomechanical analysis of structures with temperature-dependent material properties can be divided into two classes of analyses:

1. Endpoint analysis

2. Incremental analysis.

In the endpoint analysis the structure is usually considered stress- and strain-free at some initial state. Then a temperature field is imposed on the structure and the material properties in the structure are calculated. Because the structure can be subjected to temperature gradients, material properties may vary within the structure, even if the structure is composed of only one material. Once the material properties are known, displacements and stresses are calculated in the same manner as for temperature-independent analysis. Many of the investigations that include temperature-dependent material properties fall into this class. Examples are the papers Allen [2], Chen, et al. [11], and Kalam [34]. A modification to the endpoint analysis is used in buckling problems. Here an initial solution is calculated based on temperature-independent properties. Then the temperature-independent solution is used as an initial guess for an iterative procedure to find the buckling load for 
temperature-dependent properties $[12,13,35]$. The endpoint analysis, however, cannot be used for structures to which coatings are applied at different temperatures, as in the present research, or structures in which plasticity or creep effects need to be considered.

With an incremental analysis, an appropriate incremental constitutive law has to be used. This incremental constitutive law needs to account for the coupling between the stress state and the temperature dependence of the material properties. Hahn and Pagano [26] in an analytical investigation of curing stresses in laminates, and Griffin [24] in a finite element analysis of curing stresses, showed that neglecting this coupling term can lead to significant errors in the results. Hsu [30] derived finite element equations for elastic-plastic and creep problems including this coupling term for isotropic materials. A more detailed discussion of the incremental constitutive equations will be given in Chapter 2 .

An analysis of the high temperature thermomechanical behavior of Carbon/Phenolic and $\mathrm{C} / \mathrm{C}$ composites was conducted by McManus and Springer [47,48]. They included the effects of pressure distribution, volatile and vapor formation, and char formation in their model for stress and strain analysis of the composite. However, they did not study coatings and used only simple geometries.

Functional gradient materials have been analyzed in several different contexts. Analytic solutions based on concentric cylinder models were derived for FGMs as fiber coatings to evaluate the possible reduction of stresses in the matrix. For example, Jayaraman and Reifsnider [32] investigated the effect of spatially varying material properties in the interphase region between fiber and matrix on the stress distribution in fiber and matrix, and Pindera, et al. [60] studied the thermoplastic response of metal matrix composites with functionally graded interfaces. Finite element analyses of FGMs as thermal barrier coatings were conducted by Mendelson and McKechnie [50] and by Fuchiyama, et al. [21]. Mendelson and McKenzie assumed a two-dimensional axisymmetric cylindrical geometry to model a turbine vane subjected to a gas temperature of $2140^{\circ} \mathrm{K}$ on the hot surface and a cooling air temperature of $775^{\circ} \mathrm{K}$ on the other surface. In their analysis they approximated the functional gradient material by several progressive constant property layers. They investigated differ- 
ent material property variations in the FGM and found that all material property variations reduced the steady state vane temperature compared to an uncoated vane. An exponential gradient layer design yielded the lowest tensile stress in the coatings. Fuchiyama, et al. [21] analyzed a circular plate with material properties varying through the thickness of the plate from a ceramic material at the top surface to a metal at the bottom surface. They studied the nature of cracks in this plate as it was subjected to heating and cooling at the top surface. They found that small through-the-thickness cracks occurred on the ceramic side of the plate. In a series of articles Williamson, et al. [77], Drake, et al. [18], and Williamson and Rabin [76] analyzed thermal stresses in graded $\mathrm{Ni}-\mathrm{Al}_{2} \mathrm{O}_{3}$ interfaces. They considered axisymmetric disk and rod specimen geometries. The graded microstructures was treated as a series of perfectly bonded layers, each having slightly different material properties. In [77] Williamson, et al. showed that plasticity is important in realistically assessing stress reductions. In [18] and [76] it was shown that the stress reduction strongly depended on the thickness and the compositional profile of the interface layer. For some compositional profiles and thicknesses, the stresses in specimens with graded interfaces were higher than the stresses in specimens without graded interfaces. Stress and plastic strain reductions were observed for thick interlayers and composition profiles that avoided large property gradients in areas exhibiting high moduli and little plasticity. Their analysis was restricted to isotropic materials.

Aboudi, et al. [1] used a micromechanical theory to study the response of functionally graded metal matrix composites subjected to thermal gradients. In their micromechanical model they accounted for variable fiber spacing as a means to create FGMs. Their results show that it is possible to obtain more favorable stress distributions by appropriately spacing fibers in the through-the-thickness direction than by using uniform materials.

\subsubsection{Objectives of the Present Research}

From the literature review in the preceding section it can be seen that there has been analytical work conducted on different aspects of the response of $\mathrm{C} / \mathrm{C}$ composites at high tem- 
peratures. However, none of this work has included the combined effects of substrates with geometric discontinuities, FGMs, and multiple coatings in an analysis with temperaturedependent material properties and orthotropic substrates and coatings. Hence, the objectives of the present work are to:

- include temperature-dependent material properties in the analysis of coated structures,

- study the influence of including such properties,

- characterize the influence of geometric discontinuities, and

- characterize the influence of multilayer coatings and FGM coatings.

The analysis tool of choice is the finite element method because it can easily handle geometric discontinuities. Because only ceramic coatings that do not plastically deform are considered, plastic deformation was neglected in the present study. It should also be noted that failure was not addressed explicitly, i.e., by including a failure criterion. This approach was taken because the focus of the work was on the influence of various parameters on stresses. Implicit in this is the fact that anything that lowers the stresses, e.g. gradient coatings, is always desirable from the point of view of failure, and anything that increases stresses, e.g., geometric discontinuities, is always undesirable. This approach to "studying failure" is felt to be as valuable as predictions of, for example, failure temperatures based on some criterion, for example, cracks forming in the coatings.

The research focuses on a particular $\mathrm{C} / \mathrm{C}$ component with several layers of coatings, as illustrated in Fig. 1.3. Each coating can either consist of a single material or can be a FGM coating with continuously varying properties through its thickness. The corner represents a frequently encountered change in geometry that leads to stress concentrations. The $\mathrm{C} / \mathrm{C}$ component in the figure represents the cross-section of a beam, plate, or other structural component. It is assumed that none of the dimensions, material properties, nor the temperature vary with the out-of-page coordinate. This categorizes the problem as one 


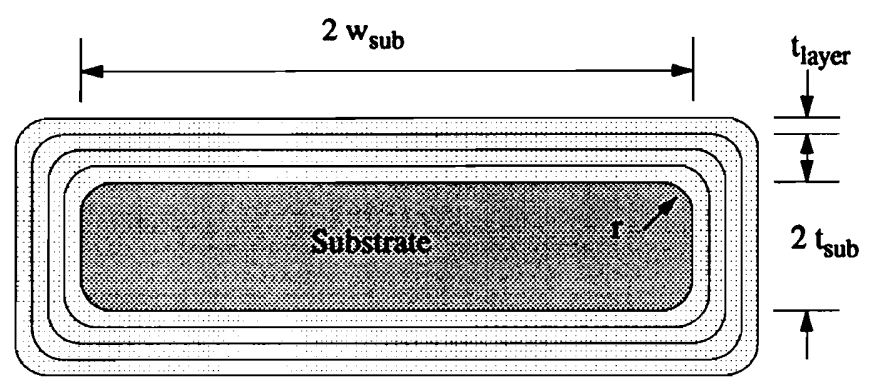

Figure 1.3: Geometry of $\mathrm{C} / \mathrm{C}$ substrate with several coatings.

of generalized plane-strain. The typical range of dimensions for the geometry shown in Fig. 1.3 is

$$
\begin{gathered}
0.0025 \leq t_{\text {layer }} \leq 0.4 \mathrm{~mm} \\
1 \leq 2 t_{\text {sub }} \leq 6.25 \mathrm{~mm} \\
2.5 \leq 2 w_{\text {sub }} \leq 300 \mathrm{~mm} \\
0.025 \leq r \leq 3.175 \mathrm{~mm}
\end{gathered}
$$

Here $2 t_{s u b}$ is defined as the substrate thickness, $2 w_{s u b}$ is defined as the substrate width, $r$ is the radius of the corner, and $t_{\text {layer }}$ is the thickness of a coating. The substrate being modeled represents a cross-ply composite with fibers oriented left-right in Fig. 1.3, and perpendicular to the page. The substrate is thus orthotropic in the cross section of Fig. 1.3. Because interest focuses on the coatings, the substrate is modeled as an equivalent homogeneous solid. Details of the microstructure of the $\mathrm{C} / \mathrm{C}$ substrate are ignored. 


\subsection{Overview of the Dissertation}

In Chapter 2, a typical problem for the analysis of $\mathrm{C} / \mathrm{C}$ substrates with coatings is presented. The importance of using incremental constitutive equations is described and the incremental constitutive equations are derived. These incremental constitutive equations are then used in the derivations of the generalized plane-strain finite element equations.

Simplified analysis models based on the incremental constitutive equations are derived in Chapter 3. These models are an incremental simplified plane-stress analysis and an incremental elasticity solution for axisymmetric concentric cylinders. Both of these simplified models allow for application of coatings at different temperatures. The simplified models are important as they are used to verify to a large degree the finite element model. Also, the incremental simplified plane-stress analysis can be used to predict stresses in coatings away from geometric discontinuities and the axisymmetric elasticity solution can be used to predict stresses in coatings on fibers.

In Chapter 4, the test cases used to verify the simplified analyses and the finite element model are described. Both simplified models were compared to standard solutions for cases with temperature-independent material properties. The finite element model was then verified by comparing results obtained using the model to results obtained using both the incremental simplified plane-stress analysis and the incremental axisymmetric elasticity solution. Further, for temperature-independent material properties, the finite element model for a gradient coated fiber was compared with an exact solution available in literature. Finally, for a realistic geometry and temperature-dependent material properties, the solution obtained using the finite element model for an approximation of a gradient coating by using an increasing number of constant property coatings with progressively different properties was compared to the finite element solution with a single gradient coating wherein the properties varied continuously.

In Chapter 5 results from temperature-independent analyses are presented. These results are used to introduce the reader to characteristics of the stress distribution in the 
coatings, especially in the vicinity of geometric discontinuities. The studies presented in Chapter 5 are a convergence study, a study of the influence of the corner region on stresses, including a limited study of the effects of coating moduli on the influence of the corner region stresses, a study of the influence of substrate orthotropy on stresses, and a study of the influence of the corner radius on the stresses in the coatings and the substrate.

Results from temperature-dependent analyses of a $\mathrm{C} / \mathrm{C}$ substrate with a $\mathrm{SiC}$ coating and of a $\mathrm{C} / \mathrm{C}$ substrate with a $\mathrm{B}_{4} \mathrm{C}$ and $\mathrm{a} \mathrm{Si}_{3} \mathrm{~N}_{4}$ coating applied at different temperatures are presented in Chapter 6. For both cases, room temperature stresses in the far-field and in the corner regions are discussed in detail. It is shown that both compensating/compliant coatings and gradient coatings next to the substrate can be used to reduce stresses in the outer oxidation-protection coatings. Further, the effects of material properties and orthotropy of the compliant coatings on the stresses are presented, and the distribution of the material properties through the thickness of the gradient coatings is shown to have a significant effect on the stresses. For the case of applying coatings at different temperatures, the effect of varying the application temperature of the coatings on the stresses in the coatings is discussed.

In Chapter 7, the important results from the studies in Chapters 5 and 6 are summarized. Based on these results, conclusions are drawn with emphasis on the nature of the stress distributions in the substrate and coating and the efficiency of stress reduction concepts. Recommendations for future work are outlined.

All temperature-dependent material properties used in this research are given in Appendix A. The effect of different forms of incremental constitutive equations on the stresses in a simple clamped-clamped beam are discussed in Appendix B. Equations for the transformation of the stiffness tensor of an orthotropic material from the material coordinate system to a global coordinate system are given in Appendix C. In Appendix D a brief outline of the implementation of the finite element model and the solution procedure are given. It is explained how the temperature-dependent material properties and the gradient coating properties are evaluated. 


\section{Chapter 2}

\section{Incremental}

\section{Temperature-Dependent Finite Element Analysis}

In this chapter the incremental temperature-dependent finite element analysis model is derived. The finite element model is a generalized plane-strain model based on linear elasticity. A typical cross section of the $\mathrm{C} / \mathrm{C}$ structure considered in the present research was shown in Fig. 1.3. It is assumed that the temperature distribution is spatially uniform. In a typical problem the stress- and strain-free temperature for a $\mathrm{C} / \mathrm{C}$ substrate is given, and the temperatures at which the coatings are to be applied to the substrate are specified. The stress and strain states of the substrate and coatings at some other specified temperature are desired. It is assumed that a coating is free of stress and strain at its application temperature, and that during the application of a new coating the states of the substrate and the previously applied coatings do not change. The individual steps in the analysis sequence are indicated by circled numbers in Fig. 2.1. In the first step, the substrate is heated from its stress- and strain-free temperature $T_{0}$ to the temperature at which the first coating will be added. Here a $\mathrm{B}_{4} \mathrm{C}$ coating will be added at $1000^{\circ} \mathrm{C}$. During this process 
the substrate experiences free thermal expansion and no overall stresses are generated in the substrate. Therefore, this step can usually be omitted in the numerical analysis. In the second step, the coating is added to the substrate. It is assumed that the stress-free state of the substrate does not change during the application of the coating. The coating is assumed stress- and strain-free when it is applied. This stress- and strain-free temperature is the reference temperature for a particular coating, denoted as $T_{r e f}$. Specifically, in this example the reference temperature for the $\mathrm{B}_{4} \mathrm{C}$ coating is $T_{\text {ref, } B_{4} C}=1000^{\circ} \mathrm{C}$. In the third step, the substrate and the $\mathrm{B}_{4} \mathrm{C}$ coating are heated to the application temperature of the next coating, here $1500^{\circ} \mathrm{C}$. Because of the thermal expansion mismatch between substrate and coating, strains and stresses are generated in the substrate and the $\mathrm{B}_{4} \mathrm{C}$ coating during heating to $1500^{\circ} \mathrm{C}$. At $1500^{\circ} \mathrm{C}$ the next coating, here $\mathrm{Si}_{3} \mathrm{~N}_{4}$, is added over the $\mathrm{B}_{4} \mathrm{C}$ coating. Again, it is assumed that during the application of the $\mathrm{Si}_{3} \mathrm{~N}_{4}$ coating the stresses and strains in the $\mathrm{B}_{4} \mathrm{C}$ coating and the substrate do not change. Also, the $\mathrm{Si}_{3} \mathrm{~N}_{4}$ coating is assumed to be free of stress and strain. The reference temperature for this coating is $T_{r e f_{,} \mathrm{Si}_{3} \mathrm{~N}_{4}}=1500^{\circ} \mathrm{C}$. Finally in the fifth step in this example, the substrate and the two coatings are cooled to the analysis temperature at which stresses and strains are desired. The analysis temperature is denoted as $T_{a}$. Here $T_{a}=20^{\circ} C$, or room temperature. Due to the fact that coatings may be applied to the $\mathrm{C} / \mathrm{C}$ substrate at different temperatures, it is not possible to use the endpoint analysis as described on page 12 in Chapter 1. Instead, an incremental formulation of the governing equations is necessary that accounts for the coupling between the stress state and the temperature-dependence of the material properties [24-26,30]. This incremental formulation is based on incremental constitutive equations. These equations are discussed in the next section.

\subsection{Incremental Constitutive Equations}

Two approaches for deriving the incremental constitutive equations for linear elastic materials with temperature-dependent properties are discussed. The first approach is to assume 

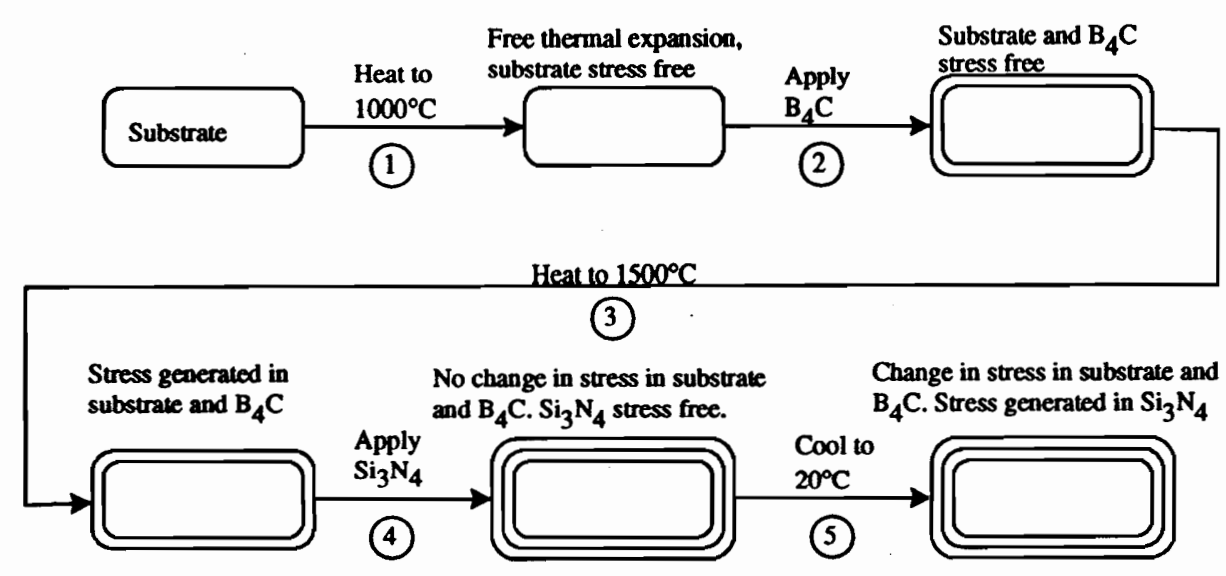

Figure 2.1: A typical problem for the analysis of a $\mathrm{C} / \mathrm{C}$ substrate with coatings.

a functional form of the mechanical strain and then take its total derivative. This approach is commonly used in literature $[24-26,30,74]$ to include plasticity or creep in the analysis. If this approach is used in an analysis, then small temperature steps may be required to obtain accurate results. In the second approach the incremental equations are obtained by taking the difference of the stress states at two different temperatures. This approach yields exact increments independent of the size of the temperature step. However, it is valid only for linear elastic material behavior. In Appendix B the different approaches are compared for a simple example.

\subsubsection{Incremental Constitutive Equations Based on Functional Form of Mechanical Strain}

This approach is presented for the sake of comparison. It is the most common approach used in literature to formulate incremental constitutive equations. Usually it is used in connection with plasticity or creep. While this approach can also be used for linear elastic materials only, as discussed in this section, the second approach for the derivation of the incremental constitutive equations described in the next section is better suited for linear 
elastic materials.

The assumed form of the mechanical strain for temperature-dependent linear elasticity is

$$
\epsilon_{i j}=\epsilon_{i j}\left(\sigma_{r s}, T, C_{r s t u}^{-1}(T)\right)
$$

where $\sigma_{r s}$ is the total stress vector, $T$ is the temperature, and $C_{r s t u}^{-1}(T)=S_{r s t u}(T)$ is the inverse of the temperature-dependent stiffness tensor, or alternatively, the temperaturedependent compliance tensor. Taking the total differential yields

$$
d \epsilon_{i j}=\frac{\partial \epsilon_{i j}}{\partial \sigma_{k l}} d \sigma_{k l}+\frac{\partial \epsilon_{i j}}{\partial T} d T+\frac{\partial \epsilon_{i j}}{\partial S_{r s t u}} \frac{\partial S_{r s t u}}{\partial T} d T
$$

Noting that $\epsilon_{i j}=S_{i j k l} \sigma_{k l}$ and defining the coefficient of thermal expansion to be

$$
\alpha_{i j}(T) \equiv \frac{\partial \epsilon_{i j}}{\partial T}
$$

eq. (2.2) may be written as

$$
d \epsilon_{i j}=S_{i j k l} d \sigma_{k l}+\alpha_{i j} d T+\sigma_{k l} \frac{\partial S_{i j k l}}{\partial T} d T
$$

Equation (2.4) can be premultiplied by $C_{i j r s}$ and rearranged to obtain

$$
d \sigma_{r s}=C_{i j r s}\left(d \epsilon_{i j}-\alpha_{i j} d T-\frac{\partial S_{i j k l}}{\partial T} \sigma_{k l} d T\right)
$$

In incremental form this can be approximated by

$$
\Delta \sigma_{i j}=C_{i j k l}\left(\Delta \epsilon_{k l}-\alpha_{k l} \Delta T-\frac{\partial S_{k l r s}}{\partial T} \sigma_{r s} \Delta T\right)
$$

where, compared to eq. (2.5), the indices have been switched as $r s \rightarrow i j, i j \rightarrow k l$, and $k l \rightarrow$ $r s$. The last term in eq. (2.6) is the coupling term that is not present in the temperatureindependent analysis.

A few remarks should be made regarding this derivation of the incremental constitutive equations. The advantage of this derivation is that, as shown by $\mathrm{Hsu}$ [30], this derivation can be extended to include plasticity and creep. One disadvantage is that when the infinitesimal 
form of the constitutive equations, eq. (2.5), is approximated by the incremental form, eq. (2.6), it is not obvious at which temperature the material properties should be evaluated. That is, it is not clear if the properties should be evaluated at the beginning, center, end, or some other point in the temperature increment. Therefore, small temperature steps may have to be used to ensure accurate results.

\subsubsection{Incremental Constitutive Equations Based on Difference in Stress States}

The following derivation is only valid for linear elastic materials because the linear elastic constitutive equations

$$
\sigma_{i j}(T)=C_{i j k l}(T)\left(\epsilon_{k l}(T)-\epsilon_{k l}^{t h}(T)\right)
$$

are used. The incremental stress is defined as the difference between the stress states at two different temperatures, i.e.,

$$
\Delta \sigma_{i j} \equiv \sigma_{i j}\left(T_{n}\right)-\sigma_{i j}\left(T_{n-1}\right)
$$

where $T_{n-1}$ and $T_{n}$ indicate the beginning and the end of the temperature increment, respectively, and where from eq. (2.7),

$$
\begin{array}{r}
\sigma_{i j}\left(T_{n-1}\right)=C_{i j k l}\left(T_{n-1}\right)\left(\epsilon_{k l}\left(T_{n-1}\right)-\epsilon_{k l}^{t h}\left(T_{n-1}\right)\right) \\
\sigma_{i j}\left(T_{n}\right)=C_{i j k l}\left(T_{n}\right)\left(\epsilon_{k !}\left(T_{n}\right)-\epsilon_{k l}^{t h}\left(T_{n}\right)\right)
\end{array}
$$

Thus,

$$
\Delta \sigma_{i j}=C_{i j k l}\left(T_{n}\right)\left(\epsilon_{k l}\left(T_{n}\right)-\epsilon_{k l}^{t h}\left(T_{n}\right)\right)-C_{i j k l}\left(T_{n-1}\right)\left(\epsilon_{k l}\left(T_{n-1}\right)-\epsilon_{k l}^{t h}\left(T_{n-1}\right)\right) .
$$

Adding and subtracting $C_{i j k l}\left(T_{n}\right)\left[\epsilon_{k l}\left(T_{n-1}\right)-\epsilon_{k l}^{t h}\left(T_{n-1}\right)\right]$ and rearranging yields

$$
\begin{aligned}
\Delta \sigma_{i j}= & C_{i j k l}\left(T_{n}\right)\left[\left(\epsilon_{k l}\left(T_{n}\right)-\epsilon_{k l}\left(T_{n-1}\right)\right)-\left(\epsilon_{k l}^{t h}\left(T_{n}\right)-\epsilon_{k l}^{t h}\left(T_{n-1}\right)\right)\right] \\
& +\left(C_{i j k l}\left(T_{n}\right)-C_{i j k l}\left(T_{n-1}\right)\right)\left(\epsilon_{k l}\left(T_{n-1}\right)-\epsilon_{k l}^{t h}\left(T_{n-1}\right)\right)
\end{aligned}
$$


Defining the incremental quantities

$$
\begin{gathered}
\Delta \epsilon_{k l} \equiv \epsilon_{k l}\left(T_{n}\right)-\epsilon_{k l}\left(T_{n-1}\right) \\
\Delta \epsilon_{k l}^{t h} \equiv \epsilon_{k l}^{t h}\left(T_{n}\right)-\epsilon_{k l}^{t h}\left(T_{n-1}\right) \\
\Delta C_{i j k l} \equiv C_{i j k l}\left(T_{n}\right)-C_{i j k l}\left(T_{n-1}\right)
\end{gathered}
$$

and substituting them into eq. (2.11) results in

$$
\Delta \sigma_{i j}=C_{i j k l}\left(T_{n}\right)\left(\Delta \epsilon_{k l}-\Delta \epsilon_{k l}^{t h}\right)+\Delta C_{i j k l}\left(\epsilon_{k l}\left(T_{n-1}\right)-\epsilon_{k l}^{t h}\left(T_{n-1}\right)\right)
$$

Now consider the term $\Delta C_{i j k l}\left(\epsilon_{k l}\left(T_{n-1}\right)-\epsilon_{k l}^{t h}\left(T_{n-1}\right)\right)$ in this equation. Using the inverse of the constitutive equation, i.e.,

$$
S_{k l r s}\left(T_{n-1}\right) \sigma_{r s}\left(T_{n-1}\right)=\epsilon_{k l}\left(T_{n-1}\right)-\epsilon_{k l}^{t h}\left(T_{n-1}\right)
$$

it can be shown that

$$
\begin{aligned}
\Delta C_{i j k l}\left(\epsilon_{k l}\left(T_{n-1}\right)\right. & \left.-\epsilon_{k l}^{t h}\left(T_{n-1}\right)\right)=\Delta C_{i j k l} S_{k l r s}\left(T_{n-1}\right) \sigma_{r s}\left(T_{n-1}\right) \\
& =\left[C_{i j k l}\left(T_{n}\right)-C_{i j k l}\left(T_{n-1}\right)\right] S_{k l r s}\left(T_{n-1}\right) \sigma_{r s}\left(T_{n-1}\right) \\
& =\left[C_{i j k l}\left(T_{n}\right)-C_{i j m n}\left(T_{n}\right) S_{m n p q}\left(T_{n}\right) C_{p q k l}\left(T_{n-1}\right)\right] S_{k l r s}\left(T_{n-1}\right) \sigma_{r s}\left(T_{n-1}\right) \\
& =C_{i j k l}\left(T_{n}\right) S_{k l r s}\left(T_{n-1}\right) \sigma_{r s}\left(T_{n-1}\right) \\
& \quad-C_{i j k l}\left(T_{n}\right) S_{k l p q}\left(T_{n}\right) C_{p q m n}\left(T_{n-1}\right) S_{m n r s}\left(T_{n-1}\right) \sigma_{r s}\left(T_{n-1}\right) \\
& =C_{i j k l}\left(T_{n}\right)\left[S_{k l r s}\left(T_{n-1}\right)-S_{k l p q}\left(T_{n}\right) C_{p q m n}\left(T_{n-1}\right) S_{m n r s}\left(T_{n-1}\right)\right] \sigma_{r s}\left(T_{n-1}\right) \\
& =C_{i j k l}\left(T_{n}\right)\left[S_{k l r s}\left(T_{n-1}\right)-S_{k l p q}\left(T_{n}\right) \delta_{p r} \delta_{q s}\right] \sigma_{r s}\left(T_{n-1}\right) \\
& =C_{i j k l}\left(T_{n}\right)\left[S_{k l r s}\left(T_{n-1}\right)-S_{k l r s}\left(T_{n}\right)\right] \sigma_{r s}\left(T_{n-1}\right) \\
& =-C_{i j k l}\left(T_{n}\right) \Delta S_{k l r s} \sigma_{r s}\left(T_{n-1}\right)
\end{aligned}
$$

where the incremental compliance tensor is defined as

$$
\Delta S_{i j k l} \equiv S_{i j k l}\left(T_{n}\right)-S_{i j k l}\left(T_{n-1}\right) .
$$

Substituting eq. (2.15) into eq. (2.13) yields the final form of the incremental constitutive equations for a linear thermoelastic material with temperature-dependent properties as

$$
\Delta \sigma_{i j}=C_{i j k l}\left(T_{n}\right)\left[\Delta \epsilon_{k l}-\Delta \epsilon_{k l}^{t h}-\Delta S_{k l r s} \sigma_{r s}\left(T_{n-1}\right)\right] .
$$


No approximations have been used in this derivation. Therefore, the incremental stress is exact within the framework of linear thermoelasticity and independent of the size of the temperature increment. This, of course, presumes that the material properties, including the thermal strains, are known functions of temperature. If, as is often the case, the coefficients of thermal expansion and not the thermal strains are given as functions of temperature, thermal strains can be obtained from the coefficients of thermal expansion by integrating the coefficients of thermal expansion as

$$
\epsilon_{i j}^{t h}(T)=\int_{T_{\text {ref }}}^{T} \alpha_{i j}(\tau) d \tau .
$$

The form of the incremental constitutive equations for a linear elastic material with temperature-dependent material properties given in eq. (2.17) was used in the derivation of the analysis models in the present study.

If the partial derivative in eq. (2.6) is replaced by a difference approximation to the derivative, i.e.,

$$
\frac{\partial S_{i j k l}}{\partial T} \approx \frac{S_{i j k l}\left(T_{n}\right)-S_{i j k l}\left(T_{n-1}\right)}{T_{n}-T_{n-1}}
$$

equivalence between the first approach and the second approaches can be established. Note also that the exact increment in the thermal strain vector in the second approach, $\Delta \epsilon_{k l}^{\text {th }}$, is approximated by $\alpha_{k l} \Delta T$ in the first approach.

\subsection{Incremental Finite Element ìodel}

A generalized plane-strain finite element model for the analysis of coatings on $\mathrm{C} / \mathrm{C}$ composites is derived in this section. The model accounts for temperature-dependent material properties, gradient coatings, and allows for application of coatings at different temperatures.

Variational principles are used to derive the finite element equations. First, a general expression is derived for the variation of the incremental total potential energy. This expression is then modified to include the generalized plane-strain assumptions. 
The total potential energy including thermal effects is given by Washizu [75] as

$$
\Pi=\frac{1}{2} \iiint_{\mathcal{V}} C_{i j k l}\left(\epsilon_{i j}-2 \epsilon_{i j}^{t h}\right) \epsilon_{k l} d V-\iiint_{\mathcal{V}} B_{i} u_{i} d V-\iint_{S_{1}} \bar{T}_{i} u_{i} d S,
$$

where $B_{i}$ and $\bar{T}_{i}$ are the body forces and surface tractions, respectively. In eq. (2.20) all quantities can be functions of temperature. To obtain an expression for the incremental total potential energy, a similar approach as described in Section 2.1.2 for the derivation of the incremental constitutive equations is taken. Here the incremental total potential energy is defined as the difference in the total potential energies at temperatures $T_{n-1}$ and $T_{n}$, i.e.,

$$
\begin{aligned}
\Delta \Pi=\Pi\left(T_{n}\right)-\Pi\left(T_{n-1}\right)=\frac{1}{2} \iiint_{\mathcal{V}}\left[C_{i j k l}\left(T_{n}\right)\left(\epsilon_{i j}\left(T_{n}\right)-2 \epsilon_{i j}^{t h}\left(T_{n}\right)\right) \epsilon_{k l}\left(T_{n}\right)\right. & -C_{i j k l}\left(T_{n-1}\right)\left(\epsilon_{i j}\left(T_{n-1}\right)-2 \epsilon_{i j}^{t h}\left(T_{n-1}\right) \epsilon_{k l}\left(T_{n-1}\right)\right] d V \\
& -\iiint_{\mathcal{V}}\left(B_{i}\left(T_{n}\right) u_{i}\left(T_{n}\right)-B_{i}\left(T_{n-1}\right) u_{i}\left(T_{n-1}\right)\right) d V \\
& -\iint_{S_{1}}\left(\bar{T}_{i}\left(T_{n}\right) u_{i}\left(T_{n}\right)-\bar{T}_{i}\left(T_{n-1}\right) u_{i}\left(T_{n-1}\right)\right) d S
\end{aligned}
$$

The variation of the increment in the total potential energy then becomes

$$
\begin{aligned}
\delta \Delta \Pi=0=\delta\left(\Pi\left(T_{n}\right)-\Pi\left(T_{n-1}\right)\right)= & \iiint_{\mathcal{V}}\left[C_{i j k l}\left(T_{n}\right)\left(\epsilon_{i j}\left(T_{n}\right)-\epsilon_{i j}^{t h}\left(T_{n}\right)\right) \delta \epsilon_{k l}\left(T_{n}\right)\right. \\
& \left.-C_{i j k l}\left(T_{n-1}\right)\left(\epsilon_{i j}\left(T_{n-1}\right)-\epsilon_{i j}^{t h}\left(T_{n-1}\right)\right) \delta \epsilon_{k l}\left(T_{n-1}\right)\right] d V \\
- & \iiint \int_{\mathcal{V}}\left(B_{i}\left(T_{n}\right) \delta u_{i}\left(T_{n}\right)-B_{i}\left(T_{n-1}\right) \delta u_{i}\left(T_{n-1}\right)\right) d V \\
- & -\iint_{S_{1}}\left(\bar{T}_{i}\left(T_{n}\right) \delta u_{i}\left(T_{n}\right)-\bar{T}_{i}\left(T_{n-1}\right) \delta u_{i}\left(T_{n-1}\right)\right) d S .
\end{aligned}
$$

If the surface tractions in eq. (2.22) are written as

$$
\bar{T}_{i}\left(T_{k}\right)=\bar{\sigma}_{i j}\left(T_{k}\right) \nu_{j}, \quad k=n-1, n
$$

where $\nu_{j}$ are the direction cosines of the normals to the surfaces, and Gauss' theorem and the equilibrium equations

$$
\sigma_{i j, j}\left(T_{k}\right)+B_{i}\left(T_{k}\right)=0, \quad k=n-1, n
$$


are used, then eq. (2.22) can be rewritten in terms of the incremental quantities introduced in the previous setions as

$$
\begin{aligned}
\delta \Delta \Pi=0= & \iiint_{\mathcal{V}}\left[C_{i j k l}\left(T_{n}\right)\left(\Delta \epsilon_{k l}-\Delta \epsilon_{k l}^{t h}-\Delta S_{k l r s} \sigma_{r s}\left(T_{n-1}\right)\right) \delta \Delta \epsilon_{i j}-\Delta B_{i} \delta \Delta u_{i}\right] d V \\
& -\iint_{S_{1}} \Delta \bar{\sigma}_{i j} \nu_{j} \delta \Delta u_{i} d S
\end{aligned}
$$

where $\Delta B_{i}$ and $\Delta \bar{\sigma}_{i j}$ are defined as

$$
\begin{aligned}
\Delta B_{i} & \equiv B_{i}\left(T_{n}\right)-B_{i}\left(T_{n-1}\right) \\
\Delta \bar{\sigma}_{i j} & \equiv \bar{\sigma}_{i j}\left(T_{n}\right)-\bar{\sigma}_{i j}\left(T_{n-1}\right) .
\end{aligned}
$$

Upon substitution of the incremental constitutive law (eq. (2.17)) into eq. (2.25) the final form of the variation of the incremental total potential energy is obtained as

$$
\delta \Delta \Pi=0=\iiint_{\mathcal{V}}\left(\Delta \sigma_{i j} \delta \Delta \epsilon_{i j}-\Delta B_{i} \delta \Delta u_{i}\right] d V-\iint_{S_{1}} \Delta \bar{\sigma}_{i j} \nu_{j} \delta \Delta u_{i} d S
$$

The assumptions of the generalized plane-strain theory as discussed by Lekhnitskii [42] and implemented into a temperature-independent finite element analysis by Avery and Herakovich [8] are used in the present derivation. A Cartesian coordinate system $x, y, z$ is assumed. As shown in Fig. 2.2, the $z$-axis is parallel to the generators of the body, and consequently the $x y$-plane is coincident with any cross section of the body. In the generalized plane-strain theory it is assumed that six components of strain are independent of one coordinate, in this case the $z$-coordinate. Further, all properties and boundary conditions are assumed independent of the $z$-coordinate. The primary unknowns in this theory are the inplane displacements $u$ and $v$, and the axial strain $\epsilon_{z}$. As a consequence of the assumption that the strains, and through the constitutive laws, the stresses are independent of $z, \epsilon_{z}$ is also independent of $x$ and $y$. Because of material orthotropy for the present problem, the shear strains $\tau_{x z}$ and $\tau_{y z}$ can be shown to be zero. If body forces and surface tractions are assumed to be zero, the variation of the incremental total potential 


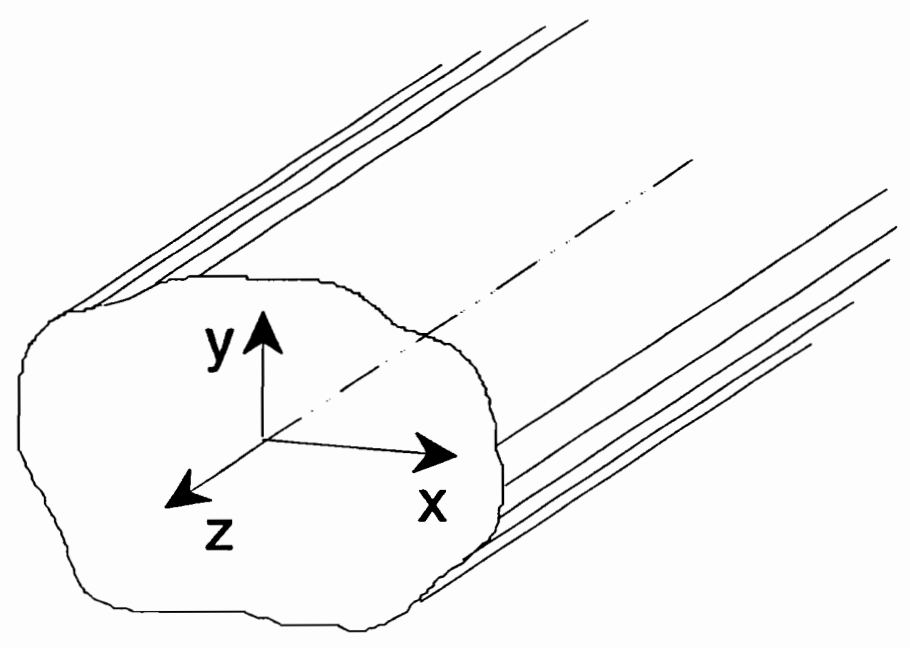

Figure 2.2: Coordinate system for the generalized plane-strain theory.

energy for the generalized plane-strain theory can be written as

$$
\delta \Delta \Pi=0=\iint_{A_{x y}}\left(\Delta \sigma_{x} \delta \Delta \epsilon_{x}+\Delta \sigma_{y} \delta \Delta \epsilon_{y}+\Delta \sigma_{z} \delta \Delta \epsilon_{z}+\Delta \tau_{x y} \delta \Delta \gamma_{x y}\right) d A-\Delta F_{z} \delta \Delta \epsilon_{z}
$$

Here $\Delta F_{z}$ is the axial force increment. 'To simplify the notation, in the following it is assumed that the components of the stiffness tensor are evaluated at $T_{n}$, i.e., $C_{i j k l}$ refers to $C_{i j k l}\left(T_{n}\right)$. The incremental stress-strain law for the generalized plane-strain theory can be written as, using contracted notation,

$$
\left\{\begin{array}{c}
\Delta \sigma_{x} \\
\Delta \sigma_{y} \\
\Delta \sigma_{z} \\
\Delta \tau_{x y}
\end{array}\right\}=\left[\begin{array}{llll}
\bar{C}_{11} & \bar{C}_{12} & \bar{C}_{13} & \bar{C}_{16} \\
\bar{C}_{12} & \bar{C}_{22} & \bar{C}_{23} & \bar{C}_{26} \\
\bar{C}_{13} & \bar{C}_{23} & \bar{C}_{33} & \bar{C}_{36} \\
\bar{C}_{16} & \bar{C}_{26} & \bar{C}_{36} & \bar{C}_{66}
\end{array}\right]\left\{\begin{array}{c}
\Delta \epsilon_{x}-\Delta \epsilon_{x}^{T} \\
\Delta \epsilon_{y}-\Delta \epsilon_{y}^{T} \\
\Delta \epsilon_{z}-\Delta \epsilon_{z}^{T} \\
\Delta \gamma_{x y}-\Delta \gamma_{x y}^{T}
\end{array}\right\},
$$


where

$$
\begin{aligned}
\Delta \epsilon_{x}^{T} & =\Delta \epsilon_{x}^{t h}+\Delta \bar{S}_{11} \sigma_{x}+\Delta \bar{S}_{12} \sigma_{y}+\Delta \bar{S}_{13} \sigma_{z}+\Delta \bar{S}_{16} \tau_{x y} \\
\Delta \epsilon_{y}^{T} & =\Delta \epsilon_{y}^{t h}+\Delta \bar{S}_{12} \sigma_{x}+\Delta \bar{S}_{22} \sigma_{y}+\Delta \bar{S}_{23} \sigma_{z}+\Delta \bar{S}_{26} \tau_{x y} \\
\Delta \epsilon_{z}^{T} & =\Delta \epsilon_{z}^{t h}+\Delta \bar{S}_{13} \sigma_{x}+\Delta \bar{S}_{23} \sigma_{y}+\Delta \bar{S}_{33} \sigma_{z}+\Delta \bar{S}_{36} \tau_{x y} \\
\Delta \gamma_{x y}^{T} & =\Delta \gamma_{x y}^{t h}+\Delta \bar{S}_{16} \sigma_{x}+\Delta \bar{S}_{26} \sigma_{y}+\Delta \bar{S}_{36} \sigma_{z}+\Delta \bar{S}_{66} \tau_{x y}
\end{aligned}
$$

and where $\bar{C}_{i j}$ and $\bar{S}_{i j}$ are the transformed stiffnesses and compliances, respectively. The transformations are given in Appendix C. The incremental strain-displacement relations are

$$
\begin{array}{ll}
\Delta \epsilon_{x}=\frac{\partial \Delta u}{\partial x} & \Delta \epsilon_{y}=\frac{\partial \Delta v}{\partial y} \\
\Delta \epsilon_{z}=\frac{\partial \Delta w}{\partial z}=\text { const. } & \Delta \gamma_{x y}=\frac{\partial \Delta u}{\partial y}+\frac{\partial \Delta v}{\partial x} .
\end{array}
$$

Substitution of eq. (2.29) and eq. (2.31) into eq. (2.28), and carrying out the steps to derive the finite element equations as described, for example, by Reddy [61], results in the following finite element equations:

$$
\left[\begin{array}{ccc}
{\left[K^{(11)}\right]} & {\left[K^{(12)}\right]} & \left\{K^{(13)}\right\} \\
{\left[K^{(21)}\right]} & {\left[K^{(22)}\right]} & \left\{K^{(23)}\right\} \\
\left\{K^{(31)}\right\} & \left\{K^{(32)}\right\} & K^{(33)}
\end{array}\right]\left\{\begin{array}{c}
\{\Delta u\} \\
\{\Delta v\} \\
\Delta \epsilon_{z}
\end{array}\right\}=\left\{\begin{array}{c}
\left\{\Delta F^{(1)}\right\} \\
\left\{\Delta F^{(2)}\right\} \\
\Delta F^{(3)}
\end{array}\right\},
$$


where

$$
\begin{aligned}
& K_{i j}^{(11)}=\iint_{A_{x y}}\left[\left(\bar{C}_{11} \frac{\partial \psi_{j}}{\partial x}+\bar{C}_{16} \frac{\partial \psi_{j}}{\partial y}\right) \frac{\partial \psi_{i}}{\partial x}+\left(\bar{C}_{16} \frac{\partial \psi_{j}}{\partial x}+\bar{C}_{66} \frac{\partial \psi_{j}}{\partial y}\right) \frac{\partial \psi_{i}}{\partial y}\right] d A \\
& K_{i j}^{(12)}=K_{j i}^{(21)}=\iint_{A_{x y}}\left[\left(\bar{C}_{12} \frac{\partial \psi_{j}}{\partial y}+\bar{C}_{16} \frac{\partial \psi_{j}}{\partial x}\right) \frac{\partial \psi_{i}}{\partial x}+\left(\bar{C}_{26} \frac{\partial \psi_{j}}{\partial y}+\bar{C}_{66} \frac{\partial \psi_{j}}{\partial x}\right) \frac{\partial \psi_{i}}{\partial y}\right] d A \\
& K_{i}^{(13)}=K_{i}^{(31)}=\iint_{A_{x y}}\left[\bar{C}_{13} \frac{\partial \psi_{i}}{\partial x}+\bar{C}_{36} \frac{\partial \psi_{i}}{\partial y}\right] d A \\
& K_{i j}^{(22)}=\iint_{A_{x y}}\left[\left(\bar{C}_{22} \frac{\partial \psi_{j}}{\partial y}+\bar{C}_{26} \frac{\partial \psi_{j}}{\partial x}\right) \frac{\partial \psi_{i}}{\partial y}+\left(\bar{C}_{26} \frac{\partial \psi_{j}}{\partial y}+\bar{C}_{66} \frac{\partial \psi_{j}}{\partial x}\right) \frac{\partial \psi_{i}}{\partial x}\right] d A \\
& K_{i}^{(23)}=K_{i}^{(32)}=\iint_{A_{x y}}\left[\bar{C}_{23} \frac{\partial \psi_{i}}{\partial y}+\bar{C}_{36} \frac{\partial \psi_{i}}{\partial x}\right] d A \\
& K^{(33)}=\iint_{A_{x y}} \bar{C}_{33} d A \\
& \Delta F_{i}^{(1)}=\iint_{A_{x y}}\left[\left(\bar{C}_{11} \Delta \epsilon_{x}^{T}+\bar{C}_{12} \Delta \epsilon_{y}^{T}+\bar{C}_{13} \Delta \epsilon_{z}^{T}+\bar{C}_{16} \Delta \gamma_{x y}^{T}\right) \frac{\partial \psi_{i}}{\partial x}\right. \\
& \left.+\left(\bar{C}_{16} \Delta \epsilon_{x}^{T}+\bar{C}_{26} \Delta \epsilon_{y}^{T}+\bar{C}_{36} \Delta \epsilon_{z}^{T}+\bar{C}_{66} \Delta \gamma_{x y}^{T}\right) \frac{\partial \psi_{i}}{\partial y}\right] d A \\
& \Delta F_{i}^{(2)}=\iint_{A_{x y}}\left[\left(\bar{C}_{16} \Delta \epsilon_{x}^{T}+\bar{C}_{26} \Delta \epsilon_{y}^{T}+\bar{C}_{36} \Delta \epsilon_{z}^{T}+\bar{C}_{66} \Delta \gamma_{x y}^{T}\right) \frac{\partial \psi_{i}}{\partial x}\right. \\
& \left.+\left(\bar{C}_{12} \Delta \epsilon_{x}^{T}+\bar{C}_{22} \Delta \epsilon_{y}^{T}+\bar{C}_{23} \Delta \epsilon_{z}^{T}+\bar{C}_{26} \Delta \gamma_{x y}^{T}\right) \frac{\partial \psi_{i}}{\partial y}\right] d A \\
& \Delta F_{i}^{(3)}=\iint_{A_{x y}}\left(\bar{C}_{13} \Delta \epsilon_{x}^{T}+\bar{C}_{23} \Delta \epsilon_{y}^{T}+\bar{C}_{33} \Delta \epsilon_{z}^{T}+\bar{C}_{36} \Delta \gamma_{x y}^{T}\right) d A+\Delta F_{z}
\end{aligned}
$$

The $K_{i j}$ are the element stiffnesses and the $\Delta F_{i}$ are the equivalent element load vectors. These finite element equations are valid for any incremental generalized plane-strain problem within the framework of the stated assumptions. The implementation of these equations, however, is specific to the problem described in the introduction to this chapter. Details of the implementation are given in Appendix D. 


\subsection{Summary}

In this chapter two derivations of incremental constitutive equations for temperature-dependent analyses were presented. One derivation is more general and to some degree classical, while the other derivation has advantages for the limitation of linear elastic behavior. While the second approach is used here, the first approach was shown to provide a comparison. The incremental generalized plane-strain finite element model derived in this chapter is based on the incremental constitutive equations obtained using the second derivation.

In the next chapter several simplified analysis tools based on the second approach are developed. These simpler tools will be used for verification purposes. 


\section{Chapter 3}

\section{Simplified Analytical Solutions}

In this chapter two simplified analytical solutions are presented. Both of these analyses are used to verify the finite element model. The first analysis is an incremental simplified plane-stress analysis and the second is an incremental axisymmetric elasticity solution. The incremental simplified plane-stress analysis could be used to predict stresses in infinitely wide composites or away from discontinuities. In the present problem this analysis is used to predict stresses away from the corner. The axisymmetric elasticity solution could also be used for the micromechanical analyses of coatings on fibers for temperature-dependent material properties.

\subsection{Incremental Simplified Plane-Stress Analysis}

According to St. Venant's principle, geometric discontinuities should affect stresses only locally. Away from geometric discontinuities, the stresses should not be influenced by the discontinuity. In the present problem the corner of the substrate with coatings represents such a discontinuity. Therefore, away from the corner the stress distributions should not be influenced by the corner. The composite without the corner resembles an infinite "laminated" plate, and therefore a plane-stress analysis can be used for stress calculations. Because of the temperature-dependent material properties and the adding of coatings at 
different temperatures, an incremental analysis has to be used. The derivation of the incremental simplified plane-stress analysis, hereafter denoted as ISPSA, follows the same steps as discussed by Jones [33] in the derivation of the classical lamination theory. The results obtained using the classical lamination theory as described by Jones will be denoted as CLT solutions. Note, however, that the coordinate system in the present derivation is differently oriented from the one used by Jones. The differently oriented coordinate system is used so that both the derivation of the incremental finite element model as discussed in Section 2.2 and the ISPSA use the same coordinate system, namely that of Fig. 2.2 on page 28 . In the ISPSA it is assumed that the through-the-thickness, or transverse, stresses $\sigma_{y}, \tau_{y z}$, and $\tau_{y x}$ are zero, and that the $y-z$ and $y-x$ cross sections remain planar after deformation. Further, in the present derivation only inplane deformations of symmetric laminates with orthotropic substrates and coatings are considered. With these assumptions, the incremental constitutive equations (2.17) for the $n$-th temperature increment of the $k$-th material layer reduce to

$$
\left\{\begin{array}{l}
{ }^{n} \Delta \sigma_{x} \\
{ }^{n} \Delta \sigma_{z}
\end{array}\right\}=\left[\begin{array}{ll}
\bar{Q}_{11} & \bar{Q}_{13} \\
\bar{Q}_{13} & \bar{Q}_{33}
\end{array}\right]^{k}\left\{\begin{array}{l}
{ }^{n} \Delta \epsilon_{x}-{ }^{n} \Delta \epsilon_{x}^{t h}-{ }^{n} \Delta S_{11}{ }^{n-1} \sigma_{x}-{ }^{n} \Delta S_{13}{ }^{n-1} \sigma_{z} \\
{ }^{n} \Delta \epsilon_{y}-{ }^{n} \Delta \epsilon_{y}^{t h}-{ }^{n} \Delta S_{13}{ }^{n-1} \sigma_{x}-{ }^{n} \Delta S_{33}{ }^{n-1} \sigma_{z}
\end{array}\right\}
$$

where $\bar{Q}_{i j}$ are the reduced transformed laminate stiffnesses evaluated at $T_{n}$. The stresses ${ }^{n-1} \sigma_{x}$ and ${ }^{n-1} \sigma_{z}$ are the residual stresses that were calculated at $T_{n-1}$. Here $T_{n}$ is the temperature at the end of the $n$-th temperature increment and $T_{n-1}$ is the temperature at the beginning of this temperature increment. To obtain the incremental force resultants, eq. (3.1) is integrated through the thickness, $h$, of the composite. These incremental force 
resultants are

$$
\begin{aligned}
\left\{\begin{array}{c}
{ }^{n} \Delta N_{x} \\
{ }^{n} \Delta N_{z}
\end{array}\right\}= & \int_{h}\left\{\begin{array}{c}
{ }^{n} \Delta \sigma_{x} \\
{ }^{n} \Delta \sigma_{z}
\end{array}\right\} d y \\
= & \int_{h}\left[\begin{array}{ll}
\bar{Q}_{11} & \bar{Q}_{13} \\
\bar{Q}_{13} & \bar{Q}_{33}
\end{array}\right] d y\left\{\begin{array}{c}
{ }^{n} \Delta \epsilon_{x} \\
{ }^{n} \Delta \epsilon_{z}
\end{array}\right\} \\
& -\int\left[\begin{array}{ll}
\bar{Q}_{11} & \bar{Q}_{13} \\
\bar{Q}_{13} & \bar{Q}_{33}
\end{array}\right]\left\{\begin{array}{c}
{ }^{n} \Delta \epsilon_{x}^{t h}-{ }^{n} \Delta S_{11}{ }^{n-1} \sigma_{x}-{ }^{n} \Delta S_{13}{ }^{n-1} \sigma_{z} \\
{ }^{n} \Delta \epsilon_{z}^{t h}-{ }^{n} \Delta S_{13}{ }^{n-1} \sigma_{x}-{ }^{n} \Delta S_{33}{ }^{n-1} \sigma_{z}
\end{array}\right\} d y \\
& {\left[\begin{array}{ll}
A_{11} & A_{13} \\
A_{13} & A_{33}
\end{array}\right]\left\{\begin{array}{l}
\Delta \epsilon_{x} \\
\Delta \epsilon_{z}
\end{array}\right\}-\left\{\begin{array}{l}
\Delta N_{x}^{T} \\
\Delta N_{z}^{T}
\end{array}\right\} }
\end{aligned}
$$

where

$$
A_{i j}=\int_{h} \bar{Q}_{i j} d y
$$

and

$$
\Delta N_{i}^{T}=\int_{h} \bar{Q}_{i j}\left(\Delta \epsilon_{j}^{t h}-\Delta S_{j r} \sigma_{r}\right) d y
$$

are the laminate stiffness matrix and the incremental equivalent resultant thermal force vector, respectively. Because in the present problem the solution for a temperature change as opposed to the solution for applied loads is sought, the incremental resultants $\Delta N_{x}$ and $\Delta N_{z}$ are set to zero in order to solve eq. (3.2) for the incremental strains $\Delta \epsilon_{x}$ and $\Delta \epsilon_{z}$. Once the strains are determined, the incremental stresses can be obtained form eq. (3.1). It should be noted that this formulation of the ISPSA allows for the application of coatings at different temperatures and for gradient coatings. The solution procedure follows the same basic steps as outlined in Appendix D for the finite element analysis. 


\subsection{Incremental Analysis of an Isotropic Axisymmetric In- finitely Long Cylinder}

In this section an incremental elasticity solution for an axisymmetric isotropic cylinder of infinite length in the $z$-direction of Fig. 2.2 is derived. The derivation accounts for temperature-dependent material properties and allows for adding of coatings to the substrate at different temperatures. The incremental constitutive equations for the $n$-th temperature increment for the $k$-th material layer of an isotropic cylinder can be written in cylindrical coordinates as

$$
\begin{aligned}
\left\{\begin{array}{l}
{ }^{n} \Delta \sigma_{r}(r) \\
{ }^{n} \Delta \sigma_{\theta}(r) \\
{ }^{n} \Delta \sigma_{z}(r)
\end{array}\right\}= & {\left[\begin{array}{lll}
C_{11} & C_{12} & C_{12} \\
C_{12} & C_{11} & C_{12} \\
C_{12} & C_{12} & C_{11}
\end{array}\right]^{k} } \\
& \left\{\begin{array}{l}
{ }^{n} \Delta \epsilon_{r}-{ }^{n} \Delta \epsilon^{t h}-{ }^{n} \Delta S_{11}{ }^{n-1} \sigma_{r}(r)-{ }^{n} \Delta S_{12}\left({ }^{n-1} \sigma_{\theta}(r)+{ }^{n-1} \sigma_{z}(r)\right) \\
{ }^{n} \Delta \epsilon_{\theta}-{ }^{n} \Delta \epsilon^{t h}-{ }^{n} \Delta S_{11}{ }^{n-1} \sigma_{\theta}(r)-{ }^{n} \Delta S_{12}\left({ }^{n-1} \sigma_{r}(r)+{ }^{n-1} \sigma_{z}(r)\right) \\
{ }^{n} \Delta \epsilon_{z}-{ }^{n} \Delta \epsilon^{t h}-{ }^{n} \Delta S_{11}{ }^{n-1} \sigma_{z}(r)-{ }^{n} \Delta S_{12}\left({ }^{n-1} \sigma_{r}(r)+{ }^{n-1} \sigma_{\theta}(r)\right)
\end{array}\right\}^{k}
\end{aligned}
$$

where the $C_{i j}$ are evaluated at $T_{n}$ and $\sigma_{r}, \sigma_{\theta}$, and $\sigma_{z}$ were calculated at $T_{n-1}$. The incremental strain-displacement equations are

$$
{ }^{n} \Delta \epsilon_{r}^{k}=\frac{d^{n} \Delta u^{k}}{d r} \quad{ }^{n} \Delta \epsilon_{\theta}^{k}=\frac{{ }^{n} \Delta u^{k}}{r} \quad{ }^{n} \Delta \epsilon_{z}=\text { const. }
$$

and the incremental equilibrium equation is

$$
\frac{d^{n} \Delta \sigma_{r}^{k}}{d r}+\frac{{ }^{n} \Delta \sigma_{r}^{k}-{ }^{n} \Delta \sigma_{\theta}^{k}}{r}=0
$$

Substitution of eqs. (3.5) and (3.6) into eq. (3.7) results in the governing equation in terms of the incremental displacements for an isotropic axisymmetric cylinder, namely,

$$
\begin{aligned}
& \frac{d^{2}\left({ }^{n} \Delta u^{k}\right)}{d r^{2}}+\frac{1}{r} \frac{d\left({ }^{n} \Delta u^{k}\right)}{d r}-\frac{{ }^{n} \Delta u^{k}}{r^{2}}
\end{aligned}
$$

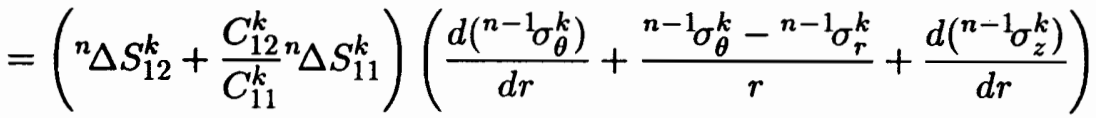

$$
\begin{aligned}
& +C_{12}^{k}{ }^{n} \Delta S_{12}^{k}\left(\frac{d\left({ }^{n-1} \sigma_{r}^{k}\right)}{d r}+\frac{d\left({ }^{n-1} \sigma_{\theta}^{k}\right)}{d r}+\frac{d\left({ }^{n-1} \sigma_{z}^{k}\right)}{d r}\right) .
\end{aligned}
$$


The general solution of this equation is

$$
{ }^{n} \Delta u^{k}(r)=\frac{{ }^{n} A_{1}^{k}}{r}+{ }^{n} A_{2}^{k} r+{ }^{n} \Delta u_{p}^{k}(r)
$$

where ${ }^{n} \Delta u_{p}^{k}(r)$ is the particular solution.

For a cylinder consisting of $N$ layers there are $2 N+1$ unknowns. These are the coefficients ${ }^{n} A_{1}^{k}$ and ${ }^{n} A_{2}^{k}$ for each layer and the incremental axial strain $\Delta \epsilon_{z}$. To solve for these unknowns, $2 N+1$ boundary and continuity conditions are needed. These conditions are:

- Finite incremental radial displacement at the center of the solid cylinder

$$
\lim _{r \rightarrow 0} u^{1}=\text { finite }
$$

- Zero incremental radial stress at the outer surface

$$
{ }^{n} \Delta \sigma_{r}^{N}(r=R)=0
$$

- Continuity of the incremental radial stresses at the layer interfaces

$$
{ }^{n} \Delta \sigma_{r}^{k}\left(r_{k}\right)={ }^{n} \Delta \sigma_{r}^{k+1}\left(r_{k}\right) \quad k=1, N-1
$$

- Continuity of the incremental radial displacements at the layer interfaces

$$
{ }^{n} \Delta u^{k}\left(r_{k}\right)={ }^{n} \Delta u^{k+1}\left(r_{k}\right) \quad k=1, n-1
$$

- Zero increment in the axial resultant force

$$
{ }^{n} \Delta N_{z}=0=2 \pi \int_{0}^{R}{ }^{n} \Delta \sigma_{z} r d r=2 \pi \sum_{k=1}^{N} \int_{r_{k-1}}^{r_{k}}{ }^{n} \Delta \sigma_{z}^{k} r d r .
$$

These boundary and continuity conditions can be used to form a system of $2 N+1$ algebraic equations for the $2 N+1$ unknowns of the problem. Once this system of equations is solved, the incremental displacements, strains, and stresses for the $n$-th increment can be calculated 
from eqs. (3.5), (3.6), and (3.9). The total quantities can then be obtained from eqs. (3.15) as

$$
\begin{aligned}
& { }^{n}{ }^{k}={ }^{n-1} u^{k}+{ }^{n} \Delta u^{k} \\
& { }^{n} \epsilon_{r}^{k}={ }^{n-1} \epsilon_{r}^{k}+{ }^{n} \Delta \epsilon_{r}^{k} \quad{ }^{n} \epsilon_{\theta}^{k}={ }^{n-1} \epsilon_{\theta}^{k}+{ }^{n} \Delta \epsilon_{\theta}^{k} \quad{ }^{n} \epsilon_{z}^{k}={ }^{n-1} \epsilon_{z}^{k}+{ }^{n} \Delta \epsilon_{z}^{k} \\
& { }^{n} \sigma_{r}^{k}={ }^{n-1} \sigma_{r}^{k}+{ }^{n} \Delta \sigma_{r}^{k} \quad{ }^{n} \sigma_{\theta}^{k}={ }^{n-1} \sigma_{\theta}^{k}+{ }^{n} \Delta \sigma_{\theta}^{k} \quad{ }^{n} \sigma_{z}^{k}={ }^{n-1} \sigma_{z}^{k}+{ }^{n} \Delta \sigma_{z}^{k} .
\end{aligned}
$$

It can be shown that if zero initial conditions are chosen, i.e., ${ }^{0} \sigma_{r}^{k}={ }^{0} \sigma_{\theta}^{k}={ }^{0} \sigma_{z}^{k}=0$, then the right hand side of eq. (3.8) is zero for all increments and therefore the particular solution is ${ }^{n} \Delta u_{p}^{k}=0$ for all $n$. For the right hand side of eq. (3.8) to be zero it is necessary that

$$
\begin{gathered}
\left(\frac{d\left({ }^{n-1} \sigma_{\theta}^{k}\right)}{d r}+\frac{{ }^{n-1} \sigma_{\theta}^{k}-{ }^{n-1} \sigma_{r}^{k}}{r}+\frac{d\left({ }^{n-1} \sigma_{z}^{k}\right)}{d r}\right)=0 \\
\left(\frac{d\left({ }^{n-1} \sigma_{r}^{k}\right)}{d r}+\frac{d\left({ }^{n-1} \sigma_{\theta}^{k}\right)}{d r}+\frac{d\left({ }^{n-1} \sigma_{z}^{k}\right)}{d r}\right)=0 .
\end{gathered}
$$

Obviously this is satisfied for the first increment, $n=1$, for which $\sigma_{r}^{k}={ }^{0} \sigma_{\theta}^{k}={ }^{0} \sigma_{z}^{k}=0$. Thus, the solution for the first increment is

$$
\begin{gathered}
{ }^{1} \Delta u^{k}(r)=\frac{{ }^{1} A_{1}^{k}}{r}+{ }^{1} A_{2}^{k} r \\
{ }^{1} \Delta \epsilon_{r}^{k}(r)=-\frac{{ }^{1} A_{1}^{k}}{r^{2}}+{ }^{1} A_{2}^{k} \quad{ }^{1} \Delta \epsilon_{\theta}^{k}(r)=\frac{{ }^{1} A_{1}^{k}}{r^{2}}+{ }^{1} A_{2}^{k} \\
{ }^{1} \Delta \sigma_{r}^{k}=\left(C_{12}^{k}-C_{11}^{k}\right) \frac{{ }^{1} A_{1}^{k}}{r^{2}}+\left(C_{11}^{k}+C_{12}^{k}\right){ }^{1} A_{2}^{k}+C_{12}^{k}{ }^{1} \Delta \epsilon_{z}-\left(C_{11}^{k}+2 C_{12}^{k}\right){ }^{1} \Delta \epsilon^{t h} \\
{ }^{1} \Delta \sigma_{\theta}^{k}=\left(C_{11}^{k}-C_{12}^{k}\right) \frac{{ }^{1} A_{1}^{k}}{r^{2}}+\left(C_{11}^{k}+C_{12}^{k}\right){ }^{1} A_{2}^{k}+C_{12}^{k}{ }^{1} \Delta \epsilon_{z}-\left(C_{11}^{k}+2 C_{12}^{k}\right){ }^{1} \Delta \epsilon^{t h} \\
{ }^{1} \Delta \sigma_{z}^{k}=2 C_{12}^{k}{ }^{1} A_{2}^{k}+C_{11}{ }^{1} \Delta \epsilon_{z}-\left(C_{11}^{k}+2 C_{12}^{k}\right){ }^{n} \Delta \epsilon^{t h} .
\end{gathered}
$$


The total stresses after the first increment are

$$
\begin{aligned}
& { }^{1} \sigma_{r}^{k}={ }^{0} \sigma_{r}^{k}+{ }^{1} \Delta \sigma_{r}^{k}={ }^{1} \Delta \sigma_{r}^{k} \\
& { }^{1} \sigma_{\theta}^{k}={ }^{0} \sigma_{\theta}^{k}+{ }^{1} \Delta \sigma_{\theta}^{k}={ }^{1} \Delta \sigma_{\theta}^{k} \\
& { }^{1} \sigma_{z}^{k}={ }^{0} \sigma_{z}^{k}+{ }^{1} \Delta \sigma_{z}^{k}={ }^{1} \Delta \sigma_{z}^{k} .
\end{aligned}
$$

Note that the derivative of the axial stress with respect to the radial coordinate $r$ is zero, i.e.,

$$
\frac{d^{1} \sigma_{z}^{k}}{d r}=0
$$

To show that the right hand side of eq. (3.8) is zero for all $n$, it is first stipulated that eqs. (3.16) - (3.17) hold for the $n$-th increment, i.e., it is assumed that

$$
\begin{gathered}
\left(\frac{d\left({ }^{n-1} \sigma_{\theta}^{k}\right)}{d r}+\frac{{ }^{n-1} \sigma_{\theta}^{k}-{ }^{n-1} \sigma_{r}^{k}}{r}+\frac{d\left({ }^{n-1} \sigma_{z}^{k}\right)}{d r}\right)=0 \\
\left(\frac{d\left({ }^{n-1} \sigma_{r}^{k}\right)}{d r}+\frac{d\left({ }^{n-1} \sigma_{\theta}^{k}\right)}{d r}+\frac{d\left({ }^{n-1} \sigma_{z}^{k}\right)}{d r}\right)=0 .
\end{gathered}
$$

Then, the solution for the $n$-th increment is

$$
\begin{gathered}
{ }^{n} \Delta u^{k}(r)=\frac{{ }^{n} A_{1}^{k}}{r}+{ }^{n} A_{2}^{k} r \\
{ }^{n} \Delta \epsilon_{r}^{k}(r)=-\frac{{ }^{n} A_{1}^{k}}{r^{2}}+{ }^{n} A_{2}^{k} \quad{ }^{n} \Delta \epsilon_{\theta}^{k}(r)=\frac{{ }^{n} A_{1}^{k}}{r^{2}}+{ }^{n} A_{2}^{k} \\
{ }^{n} \Delta \sigma_{r}^{k}=\left(C_{12}^{k}-C_{11}^{k}\right) \frac{{ }^{n} A_{1}^{k}}{r^{2}}+\left(C_{11}^{k}+C_{12}^{k}\right){ }^{n} A_{2}^{k}+C_{12}^{k}{ }^{n} \Delta \epsilon_{z}-\left(C_{11}^{k}+2 C_{12}^{k}\right){ }^{n} \Delta \epsilon^{t h} \\
-C_{11}^{k}{ }^{n} \Delta S_{11}^{k}{ }^{n-1} \sigma_{r}^{k}-\left(C_{11}^{k}{ }^{n} \Delta S_{12}^{k}+C_{12}^{k}{ }^{n} \Delta S_{11}^{k}\right)\left({ }^{n-1} \sigma_{\theta}^{k}+{ }^{n-1} \sigma_{z}^{k}\right) \\
{ }^{n} \Delta \sigma_{\theta}^{k}=\left(C_{11}^{k}-C_{12}^{k}\right) \frac{{ }^{n} A_{1}^{k}}{r^{2}}+\left(C_{11}^{k}+C_{12}^{k}\right){ }^{n} A_{2}^{k}+C_{12}^{k n} \Delta \epsilon^{n-1} \sigma_{r}^{k}+{ }^{n-1} \sigma_{\theta}^{k}+\left(C_{11}^{k-1} \sigma_{z}^{k}\right) \\
-C_{11}^{k}{ }^{n} \Delta S_{11}^{k}{ }^{n-1} \sigma_{\theta}^{k}-\left(C_{11}^{k} \Delta C_{12}^{k}\right){ }^{n} \Delta \epsilon^{t h} \\
\left.-C_{12}^{k}{ }^{n} \Delta S_{11}^{k}\right)\left({ }^{n-1} \sigma_{r}^{k}+{ }^{n-1} \sigma_{z}^{k}\right) \\
-C_{12}^{k}{ }^{n} \Delta S_{12}^{k}\left({ }^{n-1} \sigma_{r}^{k}+2^{n-1} \sigma_{\theta}^{k}+{ }^{n-1} \sigma_{z}^{k}\right)
\end{gathered}
$$




$$
\begin{aligned}
{ }^{n} \Delta \sigma_{z}^{k}=2 C_{12}^{k}{ }^{n} A_{2}^{k}+C_{11}{ }^{n} \Delta \epsilon_{z}-\left(C_{11}^{k}+2 C_{12}^{k}\right){ }^{n} \Delta \epsilon^{t h} \\
-C_{11}^{k n} \Delta S_{11}^{k}{ }^{n-1} \sigma_{z}^{k}-\left(C_{11}^{k}{ }^{n} S_{12}^{k}+C_{12}^{k}{ }^{n} \Delta S_{11}^{k}\right)\left({ }^{n-1} \sigma_{r}^{k}+{ }^{n-1} \sigma_{\theta}^{k}\right) \\
-C_{12}^{k n} \Delta S_{12}^{k}\left({ }^{n-1} \sigma_{r}^{k}+{ }^{n-1} \sigma_{\theta}^{k}+2^{n-1} \sigma_{z}^{k}\right)
\end{aligned}
$$

and the total stresses are

$$
\begin{aligned}
& { }^{n} \sigma_{r}^{k}={ }^{n-1} \sigma_{r}^{k}+{ }^{n} \Delta \sigma_{r}^{k} \\
& { }^{n} \sigma_{\theta}^{k}={ }^{n-1} \sigma_{\theta}^{k}+{ }^{n} \Delta \sigma_{\theta}^{k} \\
& { }^{n} \sigma_{z}^{k}={ }^{n-1} \sigma_{z}^{k}+{ }^{n} \Delta \sigma_{z}^{k} .
\end{aligned}
$$

By substitution it can be shown that the equations

$$
\begin{gathered}
\left(\frac{d\left({ }^{n} \sigma_{\theta}^{k}\right)}{d r}+\frac{{ }^{n} \sigma_{\theta}^{k}-{ }^{n} \sigma_{r}^{k}}{r}+\frac{d\left({ }^{n} \sigma_{z}^{k}\right)}{d r}\right)=0 \\
\left(\frac{d\left({ }^{n} \sigma_{r}^{k}\right)}{d r}+\frac{d\left({ }^{n} \sigma_{\theta}^{k}\right)}{d r}+\frac{d\left({ }^{n} \sigma_{z}^{k}\right)}{d r}\right)=0
\end{gathered}
$$

are satisfied by the stresses in eq. (3.32) and therefore the right hand side for the $(n+1)$-th increment is zero.

In summary, it has been shown that if the right hand side for the $n$-th increment is zero, then the right hand side for the $(n+1)$-th increment is zero. Further, the right hand side for the 1-st increment is zero. Thus, by induction the right hand side is zero for all $n$. The solution to the governing eq. (3.8) for all increments $n$ is therefore given by eqs. (3.27) - (3.32).

The solution procedure to a problem in which several coatings are added to a substrate at different temperatures and stresses are then calculated at prescribed temperatures is described in the following. The free thermal expansion of the substrate is only included for completeness in case the free thermal strains in the substrate are of interest. No stresses are generated in the substrate during the free thermal expansion.

1. Free thermal expansion of the substrate to the application temperature of the first coating, $T_{1}$. 
(a) Evaluate the material properties of the substrate at its stress- and strain-free temperature, $T_{0}$, and at the application temperature of the first coating, $T_{1}$.

(b) Evaluate the stiffnesses $C_{i j}\left(T_{1}\right)$, the incremental compliances ${ }^{1} \Delta S_{i j}$, and incremental thermal strains ${ }^{1} \Delta \epsilon^{t h}$.

(c) Evaluate eqs. (3.27) - (3.31), apply the boundary and continuity conditions (3.10) - (3.14), and solve for the unknowns, ${ }^{1} A_{1}^{k},{ }^{1} A_{2}^{k}$, and ${ }^{1} \Delta \epsilon_{z}$.

(d) Due to the free thermal expansion of the substrate, the incremental stresses are zero. Thus, updating the total stresses using eq. (3.32) again results in zero stresses, i.e., ${ }^{1} \sigma_{r}^{0}=0,{ }^{1} \sigma_{\theta}^{0}=0$, and ${ }^{1} \sigma_{z}^{0}=0$, where the superscript zero refers to the substrate.

(e) Store material properties of the substrate at the application temperature of the first coating, $T_{1}$, calculate and store material properties of the first coating at this temperature.

At $T_{1}$ the first coating is added to the substrate. It is assumed that this coating is stress free when it is added, thus the residual stresses are ${ }^{1} \sigma_{r}^{1}=0,{ }^{1} \sigma_{\theta}^{1}=0$, and ${ }^{1} \sigma_{z}^{1}=0$. If another coating is to be added to the substrate and the first coating, continue with step 2 . If stresses in the substrate and the first coating at some other temperature are of interest, continue with step 3.

2. Adding the $m$-th coating, $m \geq 2$.

(a) Calculate the material properties for substrate and applied coatings at the application temperature for the $m$-th coating, $T_{m}$.

(b) Evaluate the stiffnesses $C_{i j}\left(T_{m}\right)$, the incremental compliances ${ }^{m} \Delta S_{i j}$, and incremental thermal strains ${ }^{m} \Delta \epsilon^{t h}$.

(c) Evaluate eqs. (3.27) - (3.31), apply the boundary and continuity conditions (3.10) - (3.14), and solve for the unknowns, ${ }^{m} A_{1}^{k},{ }^{m} A_{2}^{k}$, and ${ }^{m} \Delta \epsilon_{z}$.

(d) Update the total stresses using eq. (3.32). 
(e) Store material properties of the substrate and the $m-1$ applied coatings at the application temperature of the $m$-th coating, $T_{m}$, calculate and store material properties of the $m$-th coating at this temperature, and store the calculated stresses.

At $T_{m}$ the $m$-th coating is added to the substrate and the $m-1$ applied coatings. It is assumed that this coating is stress free when it is added, thus the residual stresses are ${ }^{m} \sigma_{r}^{m}=0,{ }^{m} \sigma_{\theta}^{m}=0$, and ${ }^{m} \sigma_{z}^{m}=0$. If another coating is to be added to the substrate and the applied coatings, go back to step 2. If stresses in the substrate and all coatings at some other temperature are of interest, continue with step 3.

3. Analysis at prescribed temperatures, $T_{a}$.

(a) Calculate the material properties for substrate and all coatings at the analysis temperature, $T_{a}$.

(b) Evaluate the stiffnesses at $T_{a}$, the incremental compliances, and incremental thermal strains.

(c) Evaluate eqs. (3.27) - (3.31), apply the boundary and continuity conditions (3.10) - (3.14), and solve for the unknowns.

(d) Update the total stresses using eq. (3.32).

(e) Save material properties of the substrate and all coatings and the calculated stresses. Output the calculated stresses and strains.

To calculate stresses at another temperature, go back to step 3.

This procedure has been implemented in Mathematica [5]. The temperature-dependent material properties are evaluated as described in Appendix D.2. 


\subsection{Summary}

Two simplified analysis tools have been developed in this chapter. These tools will be used to verify the more complex incremental finite element analysis used in the majority of the work. 


\section{Chapter 4}

\section{Verification of the Solutions}

An extensive number of test cases were conducted to verify the solutions for the ISPSA, the incremental axisymmetric elasticity solution, and the finite element model. Both the ISPSA and the incremental axisymmetric elasticity solution were used to verify the finite element program. Therefore the verification of these models is discussed first.

\subsection{Verification of the Incremental Simplified Plane-Stress Analysis}

The tests that were used to verify the ISPSA are shown in Table 4.1. The tests are listed in order of increasing complexity. The column "Add Coatings" in the table indicates if coatings were added at different temperatures ("yes") or if all coatings were applied at the same temperature ("no"). For analyses with temperature-independent material properties (labeled "TI" in Table 4.1), the incremental constitutive equations eq. (2.17) do not depend on existing residual stresses in the composite. This significantly simplifies the ISPSA. A description of the five tests is given below. For brevity, the results for most cases are only stated and not shown. 
Table 4.1: Test cases to verify the ISPSA.

\begin{tabular}{|c|c|c|c|}
\hline Test & Properties $^{\mathrm{a}}$ & $\begin{array}{c}\text { Add } \\
\text { Coatings }\end{array}$ & $\begin{array}{c}\text { Gradient } \\
\text { Coating }\end{array}$ \\
\hline 1 & TI & No & No \\
2 & TI & Yes & No \\
3 & TI & No & Yes \\
4 & TD & No & No \\
5 & TD & Yes & Yes \\
\hline
\end{tabular}

aTI denotes temperature-independent material properties and TD denotes temperature-dependent material properties.

Test 1 For temperature-independent material properties, if all coatings are applied to the substrate at the same temperature, only one incremental analysis step for the temperature increment from the application temperature of the coatings to the prescribed analysis temperature is necessary. Therefore, for this case the ISPSA is identical to the CLT. Results obtained from the ISPSA and a CLT analysis were identical.

Test 2 For temperature-independent material properties, but with coatings being added at different temperatures, strains and stresses in the composite can be obtained by superposition of several CLT analyses. For each coating that is applied, a CLT analysis for the temperature increment from the application temperature of the previously applied coating to the application temperature of the new coating needs to be carried out. Results obtained using this superposition of CLT analyses were compared to results obtained using the ISPSA. Results were found to be identical.

Test 3 Several ISPSA analyses in which the gradient layer was replaced by an increasing number of progressively different constant-property layers were compared to a single ISPSA analysis with a gradient layer. In Fig. 4.1 it is shown that stresses calculated for an increasing number of progressively different constant-property layers converge to stresses calculated using the ISPSA with a single gradient layer. Only the $\sigma_{x}$ stress 


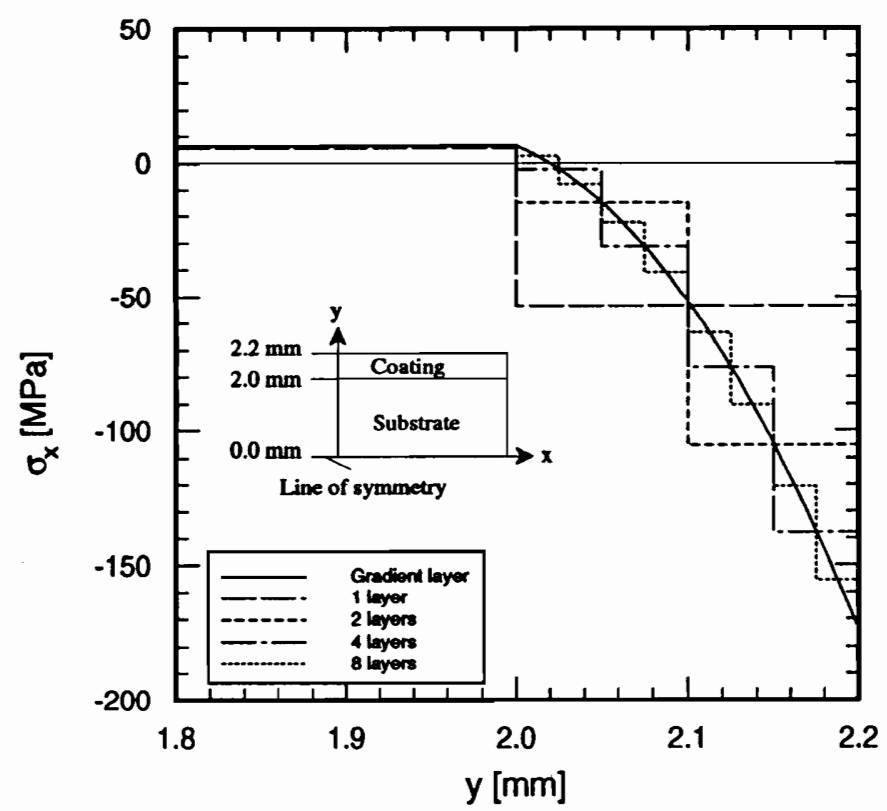

Figure 4.1: Convergence of stress calculated using the ISPSA with an increasing number of progressively different constant-property layers to stress calculated using the ISPSA with a single gradient layer.

component is shown for illustrative purposes.

Test 4 If material properties depend on temperature but all coatings are added to the substrate at the same temperature, then all coatings have the same stress- and strainfree temperature, $T_{\text {ref }}$. A CLT analysis can still be used to calculate stresses at given analysis temperatures, $T_{a}$. The input data for the CLT in this case consists of the temperature increment $\Delta T=T_{a}-T_{\text {ref }}$, the moduli and the Poisson ratio evaluated at the analysis temperature $T_{a}$, and the coefficients of thermal expansion averaged over the temperature interval. The coefficients of thermal expansion need to be averaged over the temperature increment so that the same thermal strains are used in the CLT analysis as in the ISPSA. For each analysis temperature of interest, a separate CLT analysis needs to be conducted. Stresses calculated at several temperatures using 
Table 4.2: Test cases to verify the incremental axisymmetric elasticity solution.

\begin{tabular}{|c|c|c|c|}
\hline Test & Properties $^{\mathrm{a}}$ & $\begin{array}{c}\text { Add } \\
\text { Coatings }\end{array}$ & $\begin{array}{c}\text { Gradient } \\
\text { Coating }\end{array}$ \\
\hline 1 & TI & No & No \\
2 & TI & Yes & No \\
3 & TD & No & No \\
\hline
\end{tabular}

${ }^{a} \mathrm{TI}$ denotes temperature-independent material properties and TD denotes temperature-dependent material properties. For brevity, the results for the test cases are only stated and not shown.

several CLT analyses were compared to stresses calculated using the ISPSA with temperature-dependent input data. Stresses from both analyses were found to be identical.

Test 5 Temperature-dependent results obtained using the ISPSA with a gradient coating and two additional constant-property coatings applied at different temperatures were compared to results obtained using several ISPSA analyses in which the gradient coating was replaced by an increasing number of constant-property layers with progressively different material properties. Results for an increasing number of progressively different constant-property layers converged to results for a gradient coating.

\subsection{Test Cases to Verify the Incremental Axisymmetric Elas- ticity Solution}

The test matrix for the verification of the incremental axisymmetric elasticity solution is given in Table 4.2 .

Test 1 As discussed before, if temperature-independent properties are considered and all coatings are added at the same temperature, the incremental solution simplifies sig- 
nificantly. In the case of the axisymmetric cylinder problem, the incremental solution reduces to a standard temperature-independent axisymmetric elasticity solution as described for example by Mikata and Toya [51]. Results calculated using the current program for the incremental axisymmetric elasticity solution for a cylinder with temperature-independent material properties and all coatings added at the same temperature were compared to results calculated from the standard axisymmetric elasticity solution. Identical results were obtained.

Test 2 Similar to the procedure described in Test 2 for the ISPSA, for temperatureindependent material properties and coatings added at different temperatures, superposition of standard axisymmetric elasticity analyses for each temperature increment can be used to calculate results. Again, results obtained using the current incremental model were identical to results obtained using superposition of standard axisymmetric elasticity analyses.

Test 3 The incremental axisymmetric elasticity solution was verified for temperature-dependent material properties by comparing results from this analysis to results obtained from several standard axisymmetric elasticity solutions with properties evaluated at the analysis temperatures and with an averaged coefficient of thermal expansion. The coefficient of thermal expansion was averaged over the temperature interval so that the same thermal strains were used in the standard axisymmetric elasticity solution as in the incremental axisymmetric elasticity solution. Results obtained from both analyses at prescribed temperatures were identical.

\subsection{Verification of the Finite Element Model}

A substantial number of tests was conducted to verify the finite element model. These included, among others, a comparison to a ABAQUS [4] model for temperature-independent material properties, a comparison to the the ISPSA, and several comparisons to the incre- 
mental axisymmetric elasticity solutions. All tests are described in the following sections.

\subsubsection{Comparison to ABAQUS}

Results for a substrate with two coatings applied at the same temperature and temperatureindependent material properties obtained using ABAQUS and the finite element program were compared. Identical meshes were used for both programs. Agreement was excellent.

\subsubsection{Comparison to Axisymmetric Elasticity Solutions}

\subsubsection{Comparison to the Incremental Axisymmetric Elasticity Solution}

In all of the tests described in this section, a quarter cylinder was modeled with the finite element program. Though for an axisymmetric analysis modeling any portion of the cylinder would lead to identical results, a quarter cylinder was used because the boundary conditions producing axisymmetric conditions are easiest to apply for such a model. Also, as will be seen, a quarter cylinder model resembles the corner in the problem studied here, i.e., the corner of Fig. 1.3 on page 16 (see ahead to Fig. 5.2 on page 62). A quarter cylinder mesh and the symmetry boundary conditions are shown in Fig. 4.2. The geometry, coating sequence, and coordinate system for the quarter cylinder model used in test 4 are shown in Fig. 4.3. For tests $1-3$, the gradient coating shown in the figure was not present. The gray filled region in the model indicates the gradient coating region. The radial coordinate $r$ is zero at the center of the cylinder and increases towards the outer surface. The test cases are summarized in Table 4.3. Below is a short description of the individual tests:

Test 1 Results obtained using the incremental axisymmetric elasticity solution and the finite element model for an axisymmetric cylinder with temperature-independent material properties and coatings being added at different temperatures were compared. Agreement between the results was excellent.

Test 2 An axisymmetric cylinder with temperature-dependent material properties, but all coatings applied at the same temperature, was analyzed at different temperatures 


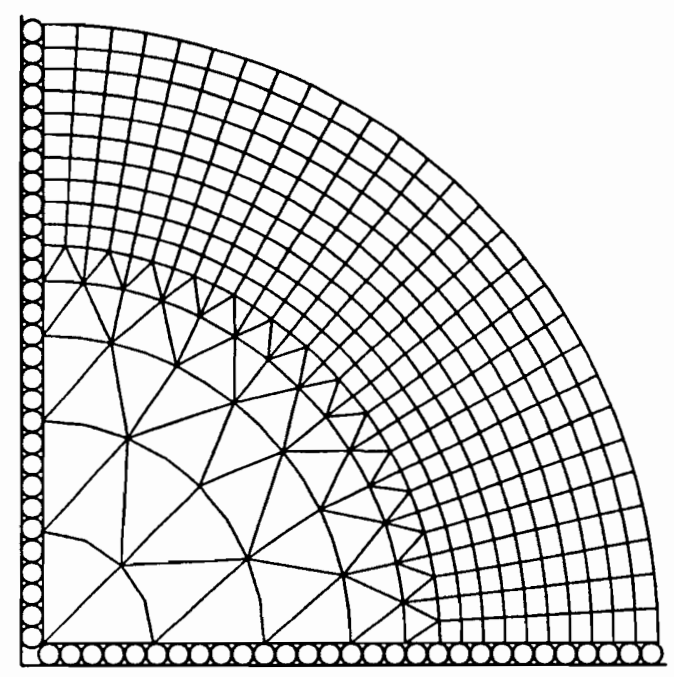

Figure 4.2: Finite element mesh and symmetry boundary conditions for the quarter cylinder model used in the verification of the finite element program.

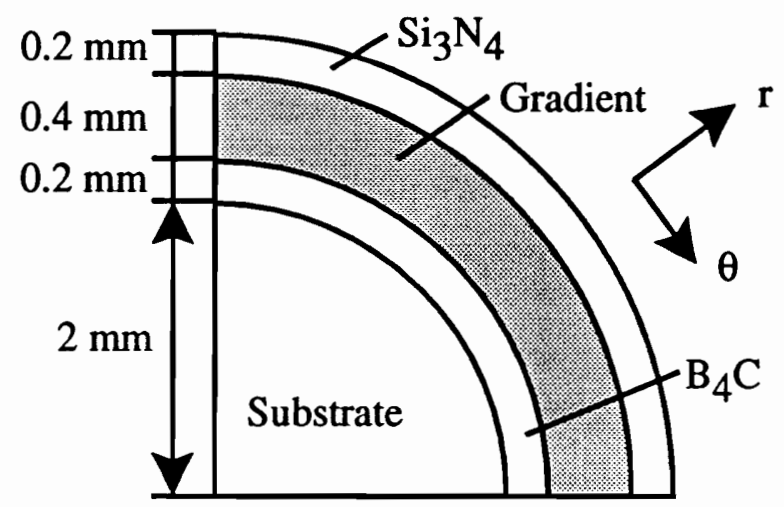

Figure 4.3: Geometry of the quarter cylinder model. 
Table 4.3: Test cases for comparison of finite element results to the axisymmetric elasticity solution.

\begin{tabular}{|c|c|c|c|}
\hline Test & Properties $^{\mathrm{a}}$ & $\begin{array}{c}\text { Add } \\
\text { Coatings }\end{array}$ & $\begin{array}{c}\text { Gradient } \\
\text { Coating }\end{array}$ \\
\hline 1 & TI & Yes & No \\
2 & TD & No & No \\
3 & TD & Yes & No \\
4 & TD & Yes & Yes \\
\hline
\end{tabular}

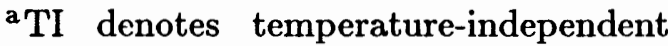
material properties and TD denotes temperature-dependent material properties.

using the incremental axisymmetric elasticity solution and the finite element model. Agreement between both solutions was excellent.

Test 3 The results from the finite element model and the incremental axisymmetric elasticity solution were compared for an axisymmetric cylinder with temperature-dependent material properties to which coatings were added at different temperatures. Excellent agreement was found between the two solutions.

Test 4 Because the incremental axisymmetric elasticity solution of Section 3.2 cannot handle gradient layers, it was checked whether or not the finite element solution with a gradient layer compared favorably with the solution the elasticity approach with an increasing number of progressive constant-property layers was converging to. As can be seen in Fig. 4.4 in which results for an analysis temperature of $1500^{\circ} \mathrm{C}$ are shown, the comparison is favorable.

Thus, from tests $1-4$, it can be concluded that the gradient layer implementation in the finite element model is correct for temperature-dependent properties and for adding coatings at different temperatures. 


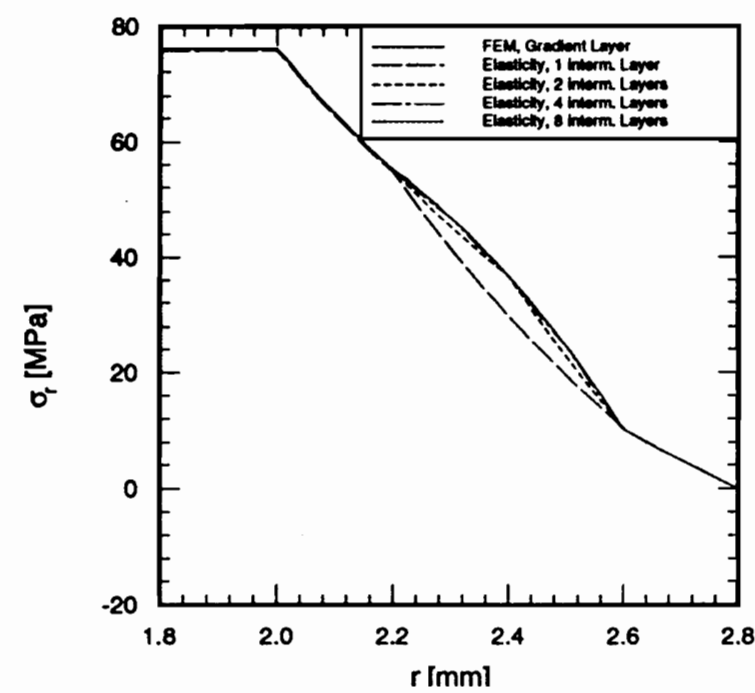

(a) $\sigma_{x}$

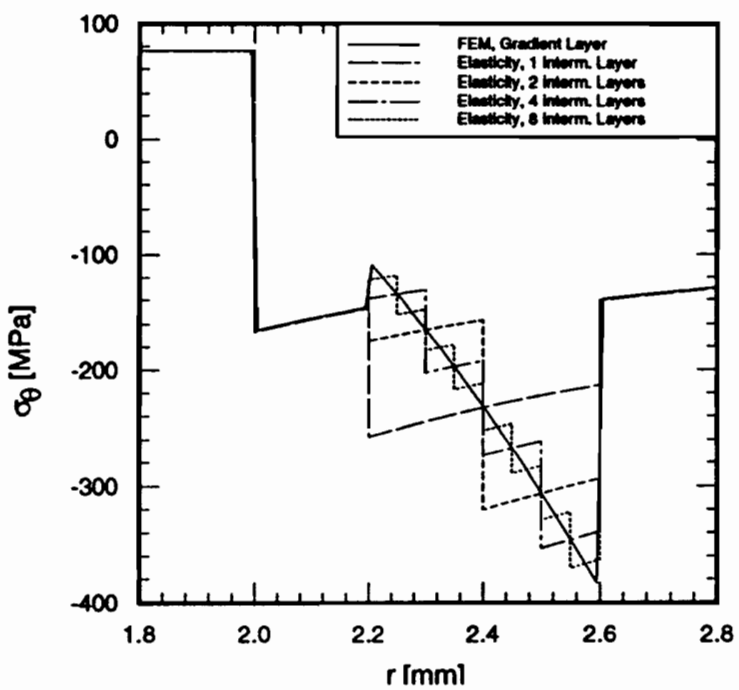

(b) $\sigma_{\theta}$

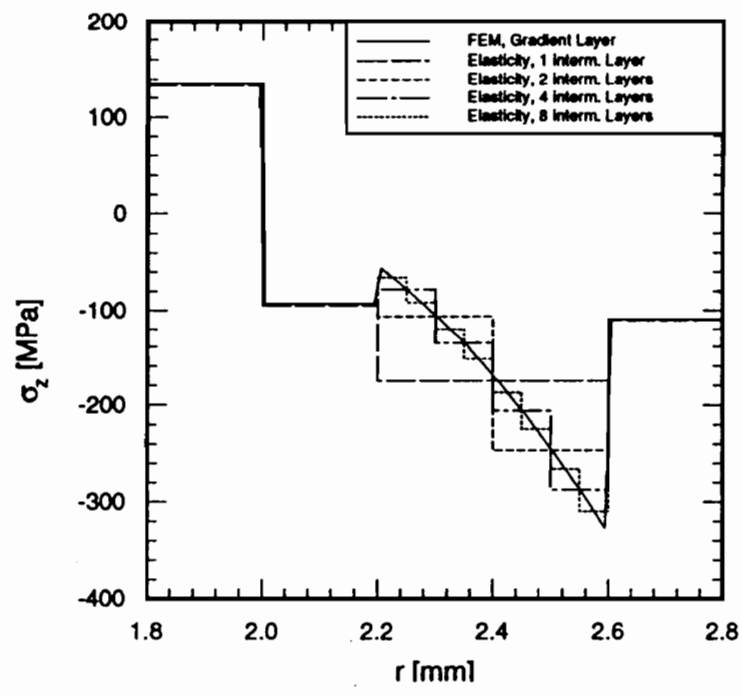

(c) $\sigma_{z}$

Figure 4.4: Comparison of the stresses calculated using the finite element program with a gradient layer (FEM) to stresses calculated using the incremental axisymmetric elasticity solution with 1, 2, 4, and 8 progressive constant-property layers (Elasticity). 


\subsubsection{Comparison to an Axisymmetric Elasticity Solution with a Gradient} Layer

Jayaraman and Reifsnider [32] derived a temperature-independent axisymmetric elasticity solution for the analysis of an assembly of concentric cylinders subjected to a uniform temperature change $\Delta T$. Properties could vary according to a power law variation through the thickness of a gradient layer. The Young's modulus and the coefficient of thermal expansion of this gradient layer were given as

$$
E=P_{y}\left(\frac{r}{r_{i}}\right)^{Q_{y}} \quad \alpha=P_{c}\left(\frac{r}{r_{i}}\right)^{Q_{c}}
$$

where $r_{i}$ is the radius of the inner surface of the gradient layer. The general solution for the radial displacement in the gradient layer was given as

$$
u(r)=A r^{m_{1}}+B r^{m_{2}}-C \nu r+\frac{\frac{1+\nu}{1-\nu} P_{c}\left(Q_{y}+Q_{c}\right) r^{Q_{c}+1} \Delta T}{\left(Q_{c}^{2}+2 Q_{c}+Q_{y} Q_{c}+Q_{y}+Q_{y} \frac{\nu}{1-\nu}\right) r_{i}^{Q_{c}}}
$$

where

$$
m_{1}, m_{2}=\frac{1}{2}\left[-Q_{y} \pm \sqrt{\frac{Q_{y}^{2}(1-\nu)-4 \nu\left(Q_{y}+1\right)+4}{1-\nu}}\right] .
$$

To further verify the finite element program, results obtained using a finite element model of a quarter cylinder and the above elasticity solution were compared for a cylinder consisting of a substrate and three coatings. The inner and outer coating had constant properties through the thickness. The center coating was a gradient coating with linear variation of properties from the properties of the inner coating to the properties of the outer coating, as illustrated in Fig. 4.3. A uniform temperature change $\Delta T$ was applied. The excellent agreement of the results obtained using both solutions is illustrated in Fig. 4.5.

\subsubsection{Comparison to the Incremental Simplified Plane-Stress Analysis}

For wide substrates the corner should not affect stresses away from the corner. Thus, stresses calculated using the finite element model should approach stresses calculated using 


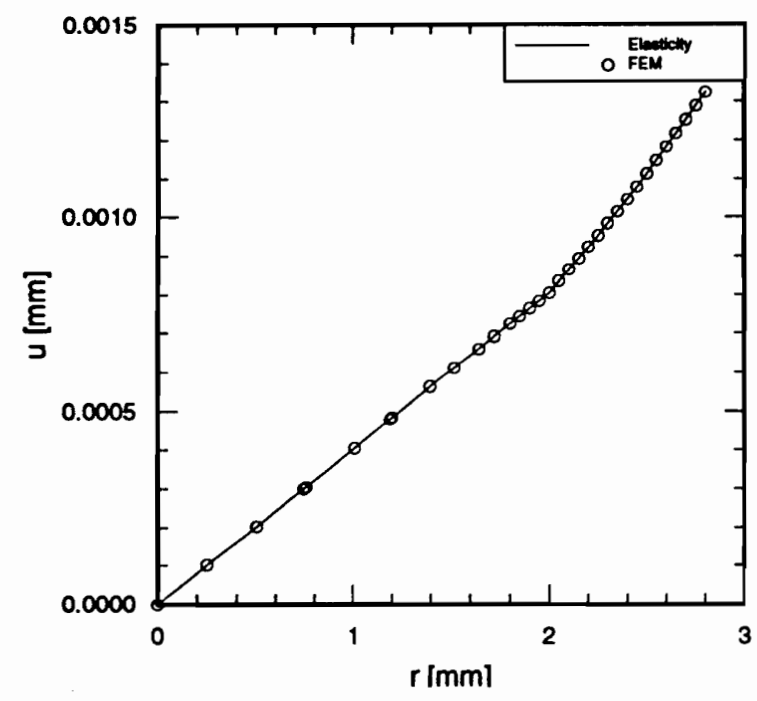

(a)

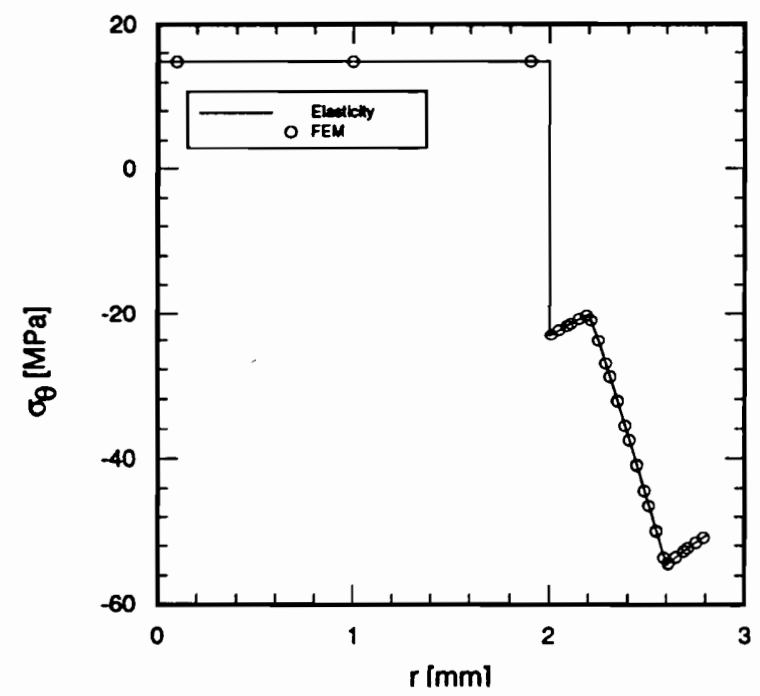

(c)

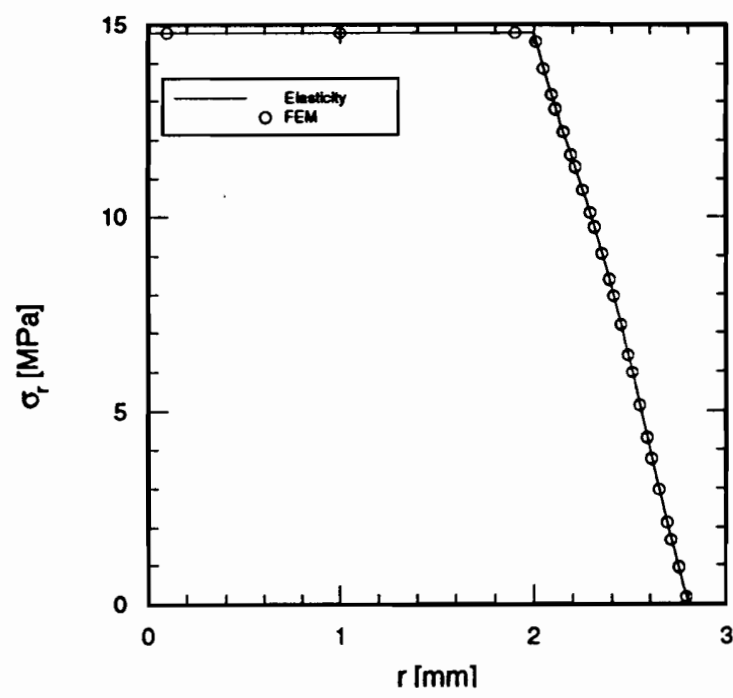

(b)

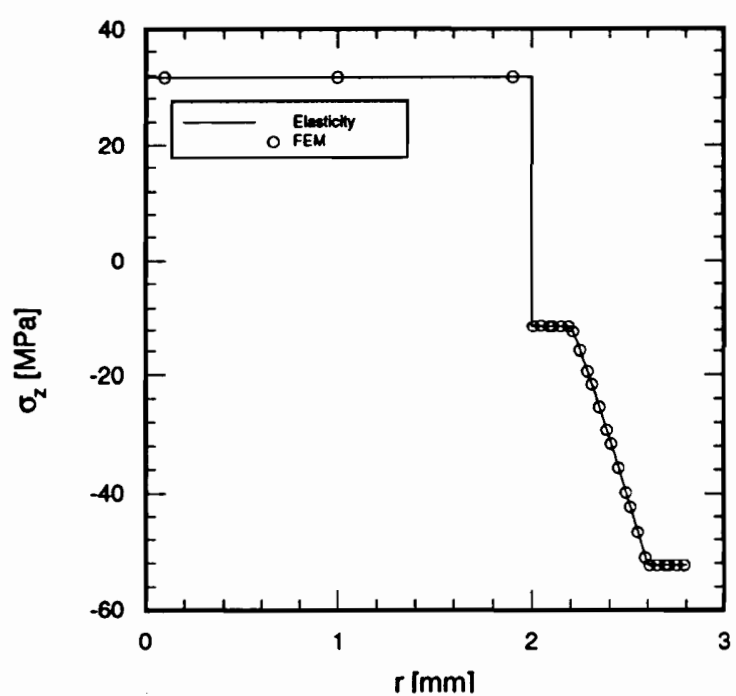

(d)

Figure 4.5: Comparison of results obtained from the finite element program and an elasticity solution for a concentric cylinder assembly including a gradient layer [32]: (a) radial displacement, (b) radial stress, (c) circumferential stress, (d) axial stress. 


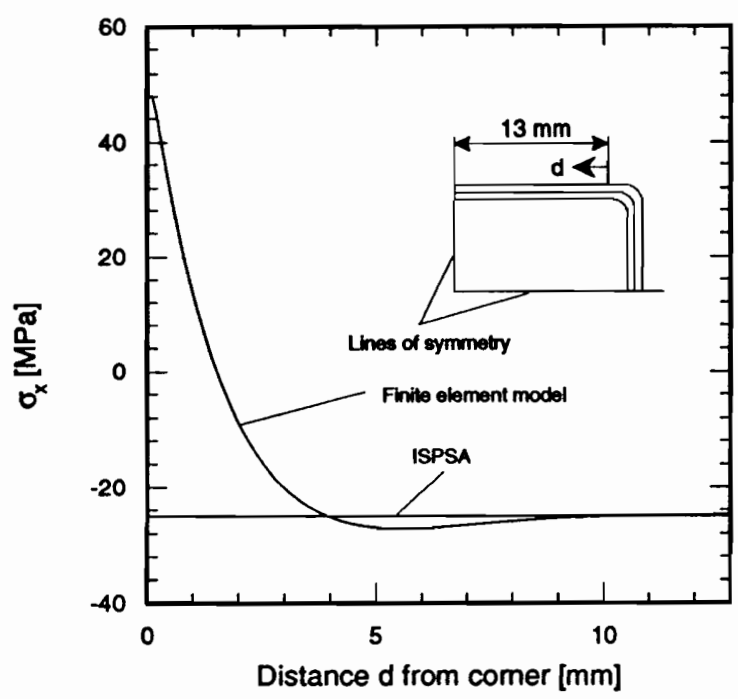

Figure 4.6: Convergence of the axial stress $\sigma_{x}$ calculated using the finite element model to the stress calculated using the ISPSA when moving away from the corner.

the ISPSA when moving away from the corner towards the vertical line of symmetry. In Fig. 4.6 the stress component $\sigma_{x}$ near the surface of the composite is shown as a function of the distance from the corner for a wide substrate with two coatings applied at the same temperature. It can be seen that when moving away from the corner, the stress calculated using the finite element model approaches the stress calculated using the ISPSA .

\subsubsection{Comparison of Finite Element Analyses}

As for the axisymmetric cylinder model, for the actual geometry of interest (Fig. 1.3) it was checked to determine if the finite element results for an approximation of gradient coatings with an increasing number of progressively different constant-property coatings would approach finite element results of the model with a gradient coating. In this test temperature-dependent material properties were considered. The gradient coating was approximated by $1,2,4$, and 8 constant-property coatings. To determine the properties of 
the constant-property layers, the gradient coating properties evaluated at the center of each constant-property coating were assigned to the constant-property coating. The dimensions of the substrate were taken as $w_{s u b}=4 \mathrm{~mm}, t_{s u b}=1 \mathrm{~mm}$, and $r=0.2 \mathrm{~mm}$, and the coating thickness was $t_{\text {coat }}=0.2 \mathrm{~mm}$. The material of the coating at the substrate-coating interface was pyrolytic carbon, and the material at the free surface of the coating was SiC. The material properties of the substrate, the pyrolytic carbon, and the SiC are given in Appendix A. The substrate and coatings were cooled by $100^{\circ} \mathrm{C}$.

In Figs. 4.7(a) - 4.7(d) the variation of the stress component $\sigma_{s}$ through the thickness of the coating is shown at different locations of the composite. The stresses are shown as a function of the thickness coordinate $t$, which is zero at the free-surface of the composite and increases as moving toward the substrate. The substrate-coating interface is at $t=0.2 \mathrm{~mm}$. For all locations it can be seen that the stresses calculated using an increasing number of constant-property layers approach the stresses calculated using the gradient coating. It also can be observed that if only one constant-property layer is used to approximate a gradient coating, not only are the maximum and minimum values of the stress in error, but also the spatial distribution of the stress is predicted incorrectly. For example in Fig. 4.7(c), while the gradient coating predicts the maximum stress $\sigma_{s}$ at the free surface of the composite, the approximation with one constant-property coating predicts the maximum stress at the substrate-coating interface.

\subsection{Summary}

In this section the ISPSA, the incremental axisymmetric elasticity solution, and the finite element solution were verified. It was shown that the finite element model correctly predicts stresses for temperature-dependent material properties, for gradient coatings, and for coatings applied at different temperatures. It further was shown that an approximation of a gradient coating by progressively different constant-property layers may predict wrong results if only few of those layers are used. 


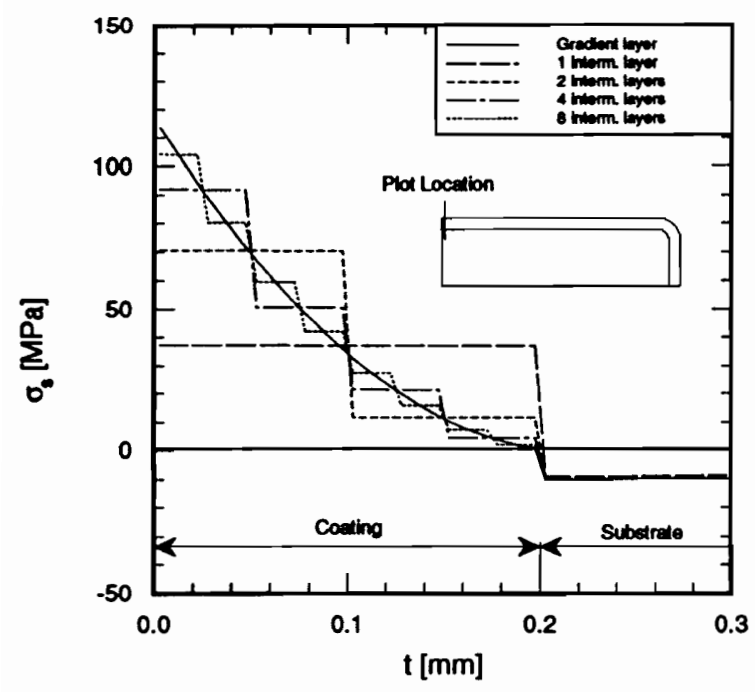

(a)

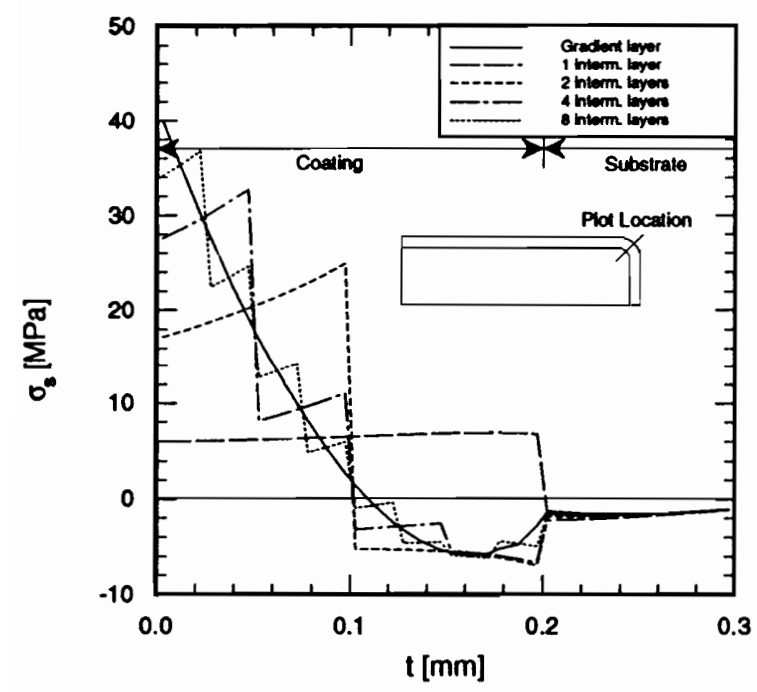

(c)

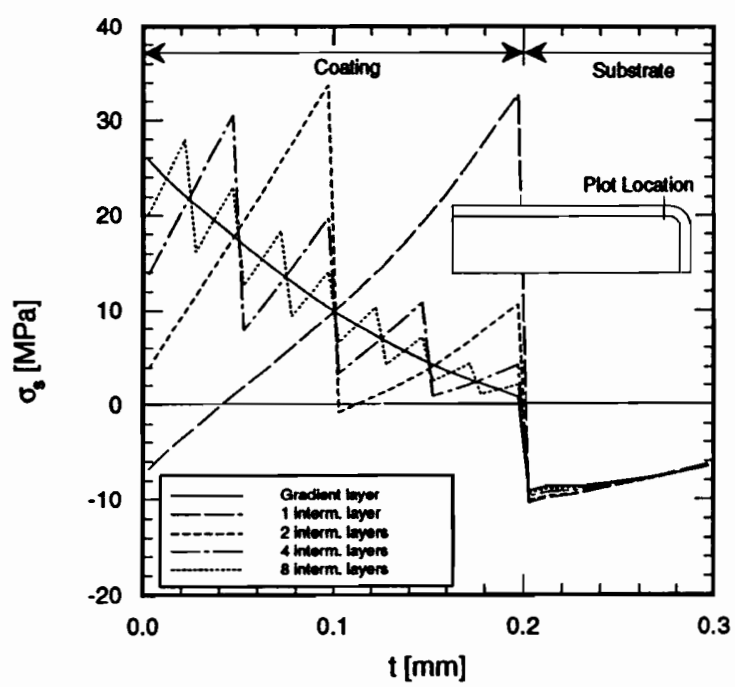

(b)

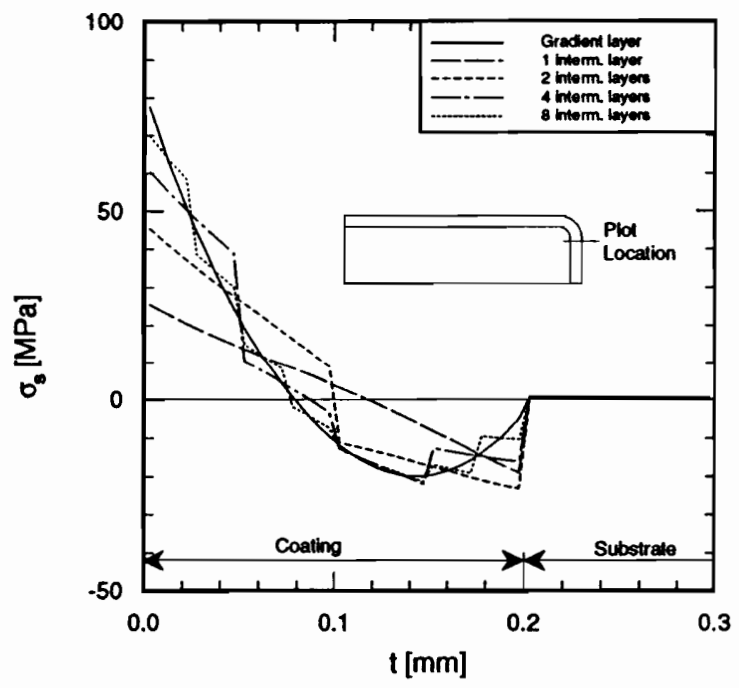

(d)

Figure 4.7: Stress distributions through the thickness of the coating and part of the substrate at different locations in the substrate. At the free surface the coordinate $t$ is zero and at the substrate-coating interface $t=0.2 \mathrm{~mm}$. 
In the chapters to follow, the finite element program is used to study the stresses in coatings on $\mathrm{C} / \mathrm{C}$ substrates. The character of the stress distributions and the influence of material and geometric parameters on the stresses are explored. 


\section{Chapter 5}

\section{Temperature-Independent Results}

In this chapter results from temperature-independent analyses of $\mathrm{C} / \mathrm{C}$ composites with coatings applied at the same temperature are presented. These analyses are used to explain features of the stress distribution in substrate and coatings that relate to the substrate orthotropy and the geometry of the composite. By considering the simplified temperatureindependent cases, the influence of substrate orthotropy and geometry can be studied without being obscured by more complicated effects such as the temperature-dependence of the material properties. Qualitatively, trends established in this chapter should occur in all problems with a similar geometry. However, quantitatively they may depend on the particular combinations of coating and substrate properties used. The parameter studies conducted are:

- a convergence study,

- a study of the effect of the width of the substrate on the stresses,

- a study of the effect of substrate orthotropy on the stresses, and

- a study of the effect of the corner radius on the stresses. 


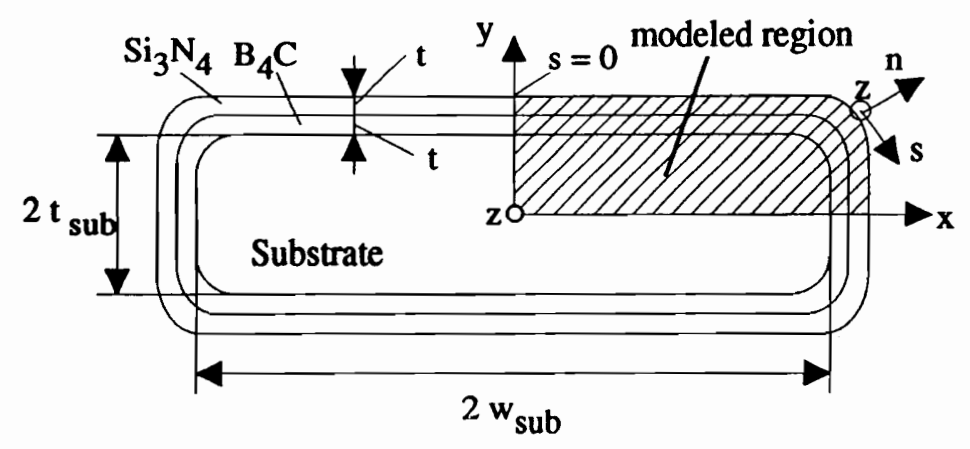

Figure 5.1: Modeled region of a $\mathrm{C} / \mathrm{C}$ substrate with two coatings and coordinate system for stress calculations.

\subsection{Representation of the Results}

A representative combination of substrate and two coatings is shown in Fig. 5.1. Because of symmetry of geometry and material properties, only a quarter of the problem, as indicated by the shading in Fig. 5.1, needs to be modeled with finite elements. Unless otherwise indicated, stresses shown in figures for results are shown as a function of the tangential $s$-coordinate and are transformed to a $s-n-z$ coordinate system. As indicated in Fig. 5.1, the $s$-direction is always tangential to the free surface of the composite, and the $n$-direction is always normal to the free surface. The $z$-direction is the out-of-page, or axial, direction. The $s$-coordinate is zero at the vertical line of symmetry of the composite and increases towards the corner. It reaches its highest value at the horizontal line of symmetry. In the discussion of results the expressions "before the corner" and "after the corner" have to be considered in relation to the $s$-coordinate. In figures to follow that illustrate stresses as a function of $s$, the quarter-circle region that constitutes the corner is indicated specifically. Also, because the width of the substrate is always at least as large as the height, the term "far-field" refers to the region of the substrate and coatings away from the corner, near the vertical line of symmetry. The stress components of interest are the tangential stress $\sigma_{s}$, 
the normal stress $\sigma_{n}$, the axial stress $\sigma_{z}$, and the shear stress $\tau_{s n}$. As mentioned previously, the other stresses, namely $\tau_{s z}$ and $\tau_{n z}$, are zero. The material data used for the analyses in this section are indicated by $a^{*}$ in the tables given in Appendix A.

\subsection{Convergence Studies}

From a comparison of typical dimensions of the substrate and coatings as given in Section 1.4.2, it can be seen that the thickness dimension of the coatings often is only a fraction of the thickness of the substrate. The size of the finite elements that can be used in the analysis depends on the thickness of the coatings. To obtain a feasible problem size, i.e., a feasible number of finite elements, the substrate needs to be modeled with much larger elements than the coatings. In the convergence studies it was investigated if the transition from the small elements in the coatings to the large elements in the substrate causes convergence problems. Further, in the corner region very large stress gradients occur both in the tangential and the transverse directions. Therefore, modeling a coating with only one element through the thickness of the coating may not yield converged results. It was also studied how many elements should be used through the thickness of a coating to obtain converged results.

For this study, a $\mathrm{C} / \mathrm{C}$ substrate with two coatings, $\mathrm{B}_{4} \mathrm{C}$ and $\mathrm{Si}_{3} \mathrm{~N}_{4}$, both applied at the same temperature and subjected to a $100^{\circ} \mathrm{C}$ temperature increase, was analyzed. The dimensions of the substrate were $w_{s u b}=4 \mathrm{~mm}, t_{s u b}=1 \mathrm{~mm}$, and $r=0.5 \mathrm{~mm}$. Because the stress gradients were expected to be largest in the corner region, an element aspect ratio of one was chosen in the corner region. The element aspect ratio is the length-to-thickness ratio of a finite element. Away from the corner the element aspect ratio was increased because the stress gradients were expected to be smaller. Convergence for three different coating thicknesses, $t_{\text {coat }}=0.05 \mathrm{~mm}, t_{\text {coat }}=0.1 \mathrm{~mm}$, and $t_{\text {coat }}=0.2 \mathrm{~mm}$ was considered. For all cases stresses were analyzed with $1,2,4$, and 8 elements through the thickness of each coating. In Table 5.1 the number of elements and nodes in the finite element meshes for the 
Table 5.1: Number of elements and number of nodes in the finite element meshes used in the convergence study.

\begin{tabular}{|c|c|c|c|c|}
\hline$t_{\text {layer }[\mathrm{mm}]}$ & $\begin{array}{c}\text { Elem. through } \\
\text { thickness }\end{array}$ & $\begin{array}{c}\text { Elem. in } \\
\text { mesh }\end{array}$ & $\begin{array}{c}\text { Nodes in } \\
\text { mesh }\end{array}$ & Skyline $^{\mathbf{a}}$ \\
\hline & 1 & 327 & 982 & 151447 \\
0.05 & 2 & 818 & 2389 & 700690 \\
& 4 & 2183 & 6354 & 3906551 \\
& 8 & 6606 & 19365 & 31132498 \\
\hline \multirow{3}{*}{0.1} & 1 & 146 & 477 & 50014 \\
& 2 & 406 & 1223 & 214144 \\
& 4 & 1113 & 3280 & 1143861 \\
0.2 & 8 & 3362 & 9903 & 7304932 \\
\hline & 1 & 96 & 319 & 32976 \\
& 2 & 191 & 616 & 79125 \\
& 4 & 561 & 1700 & 350601 \\
& 8 & 1744 & 5191 & 2043392 \\
\hline
\end{tabular}

${ }^{\mathrm{a}}$ The skyline is the dimension of the vector that stores the stiffness matrix.

different cases are shown. The skyline column in the table indicates the necessary dimension of the array that stores the assembled stiffness matrix in skyline form, as described by Bathe and Wilson [9]. It can be seen that memory requirements for the cases with a finer mesh and thin coatings may be quite substantial.

First the convergence for the case with a coating thickness of $t_{c o a t}=0.05 \mathrm{~mm}$ is discussed. For this case the finite element meshes for 1, 2, and 4 elements through the thickness of a coating are shown in Fig. 5.2. The mesh for 8 elements through the thickness is not shown because the individual elements would not be recognizable in the scale the meshes are shown. As discussed in Appendix D.1, the coatings are modeled with 8-node quadrilateral elements. The coatings and substrate are indicated in Fig. 5.2. The transition from smaller to larger elements always occurs in the substrate. Between the substrate-coating interface and the triangular element transition region four rows of 8-node quadrilateral elements are always used. Stresses in the substrate are shown along the center line of those four element rows, i.e., along the interface between the second and third rows. 


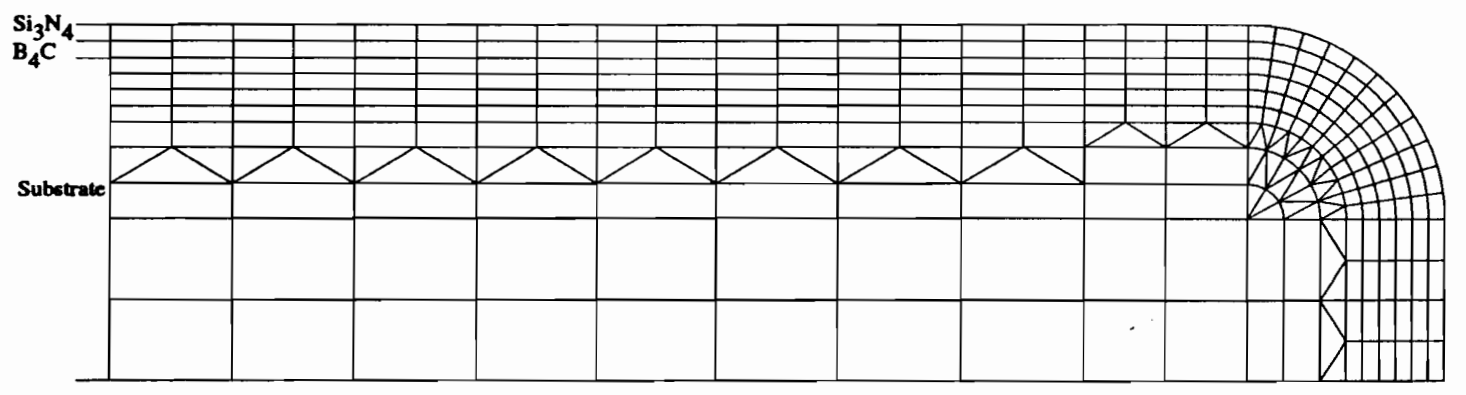

(a) 1 element through thickness of a coating.

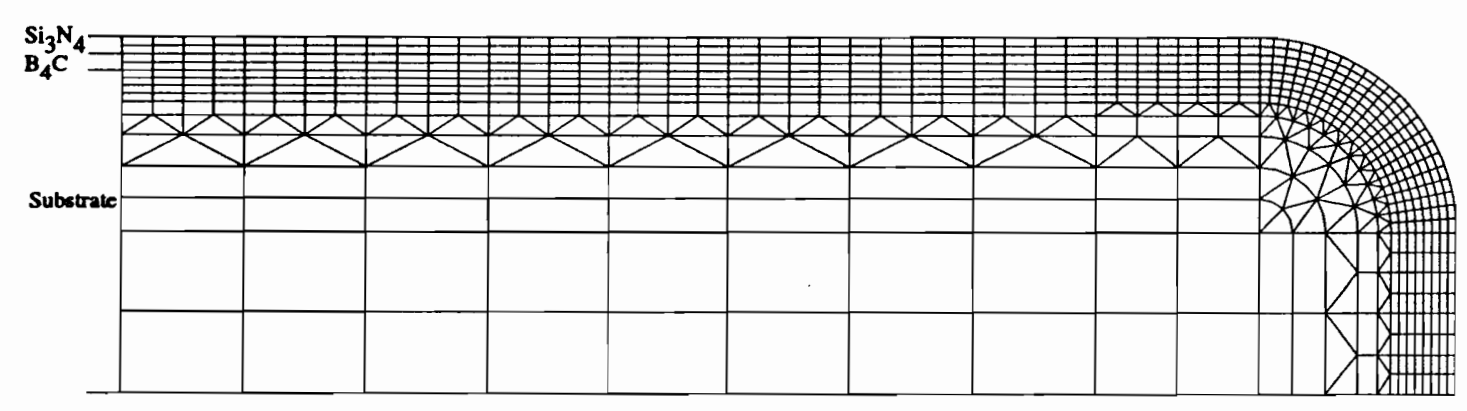

(b) 2 elements through thickness of a coating.

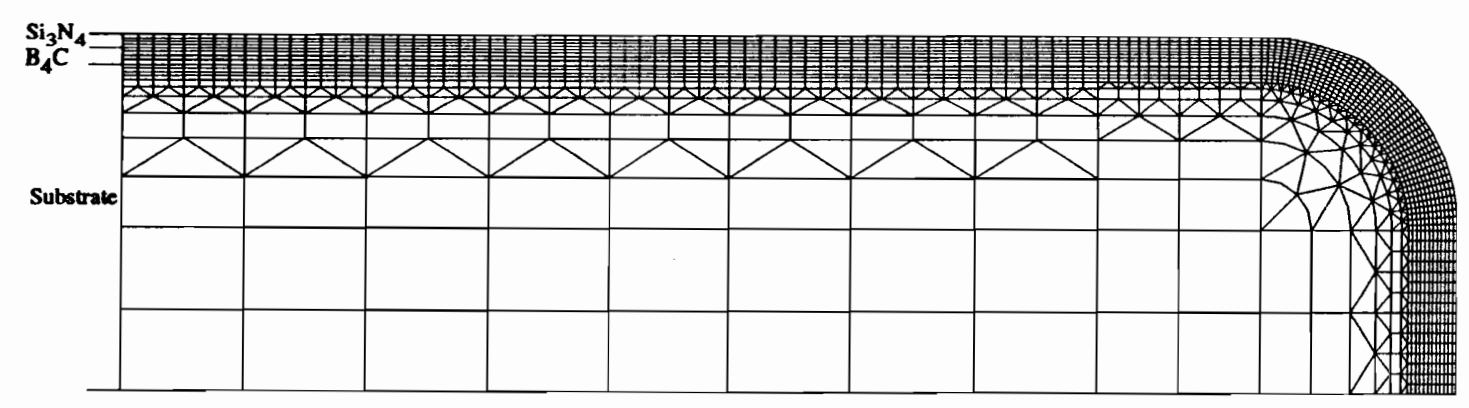

(c) 4 elements through thickness of a coating.

Figure 5.2: Finite element meshes used in the convergence study for 1, 2, and 4 elements through the thickness of a coating for $t_{\text {coat }}=0.05 \mathrm{~mm}, w_{s u b}=4 \mathrm{~mm}, t_{s u b}=1 \mathrm{~mm}$, and $r=0.5 \mathrm{~mm}$. 
In Figs. 5.3 - 5.6 the four stress components of interest, the tangential stress $\sigma_{s}$, the axial stress $\sigma_{z}$, the normal stress $\sigma_{n}$, and the shear stress $\tau_{s n}$, at the center (midlayer) of the $\mathrm{Si}_{3} \mathrm{~N}_{4}$ coating are shown. Away from the corner all stress components are converged for 2 elements through the thickness of a coating. In the corner region, the region where the largest stress gradients are present, the tangential and axial stress components are also converged for 2 elements through the thickness of a coatings. For these stress components, the oscillations of the solution for one element through the thickness is greater in the corner region than away from the corner.

For the normal and shear stresses, $\sigma_{n}$ and $\tau_{s n}, 1$ and 2 elements through the thickness of a coating do not give converged stresses in the corner region. The solutions for these cases oscillate about the solutions for 4 and 8 elements through the thickness. Almost no difference in stresses can be observed for 4 and 8 elements through the thickness. Because for the shear and normal stresses more elements through the thickness of a coating are necessary to obtain converged results than for the tangential and the axial stresses, the normal and shear stresses are the critical stress components for convergence.

To assess the dependence of the convergence on the coating thickness, the same problem as above was analyzed for different coating thicknesses. Because the normal stress is one of the critical stress components for the convergence, only results for the normal stress are shown for the thicker coating cases. In Figs. 5.7 and 5.8 the stress component $\sigma_{n}$ at the center of the $\mathrm{Si}_{3} \mathrm{~N}_{4}$ coating is shown for coating thicknesses of $0.1 \mathrm{~mm}$ and $0.2 \mathrm{~mm}$, respectively. As for the thin $0.05 \mathrm{~mm}$ coatings case, the stresses are converged away from the corner for only 2 elements through the thickness of a coating. In the corner region, 4 elements through the thickness of a coating are necessary to achieve convergence.

The influence of the transition from small to large elements in the substrate can be seen in Fig. 5.8. Careful inspection of the stress in this figure shows that for $s$-coordinates before the corner for one element through the thickness of a coating, the solution oscillates. The number of waves in the solution corresponds to the number of the largest elements in the substrate. This behavior cannot be observed if there is more than one element through 


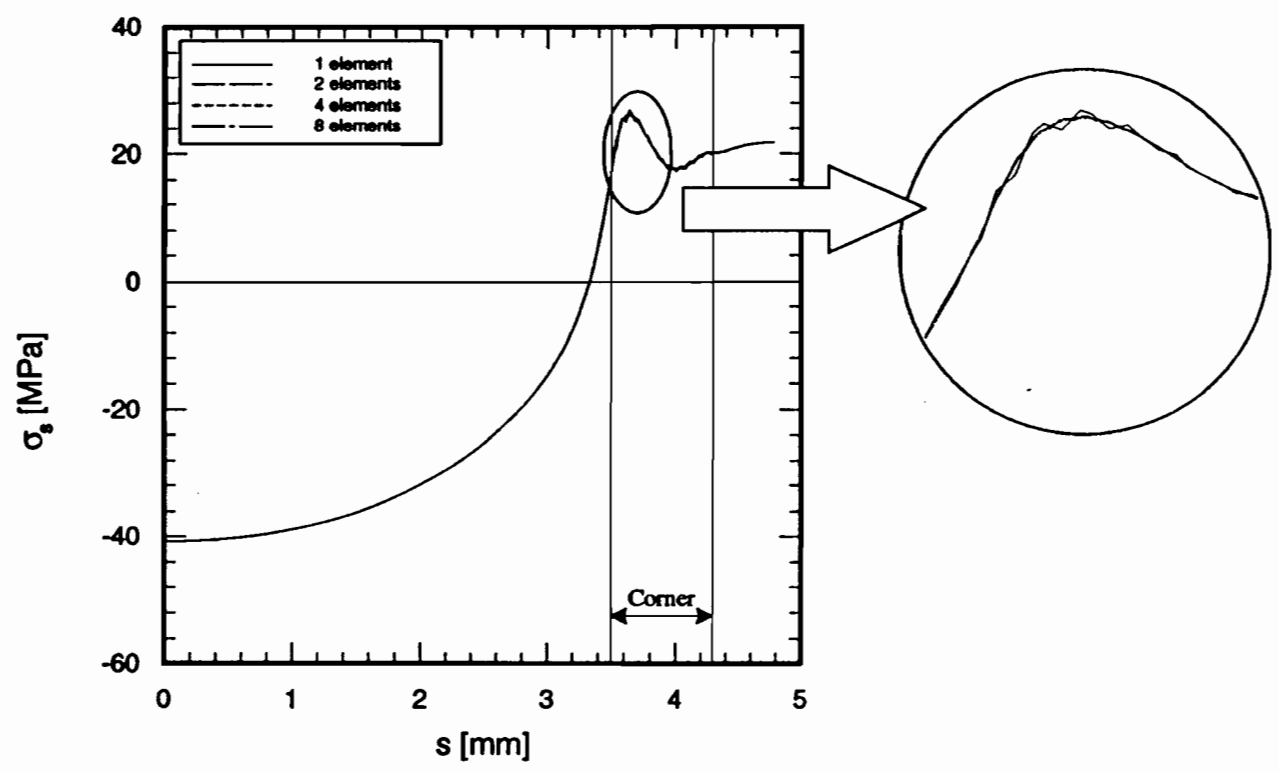

Figure 5.3: Convergence of tangential stress $\sigma_{s}$ in $\mathrm{Si}_{3} \mathrm{~N}_{4}$ coating for a substrate with two $0.05 \mathrm{~mm}$ thick coatings $\left(w_{s u b}=4 \mathrm{~mm}, t_{s u b}=1 \mathrm{~mm}, r=0.5 \mathrm{~mm}\right)$.

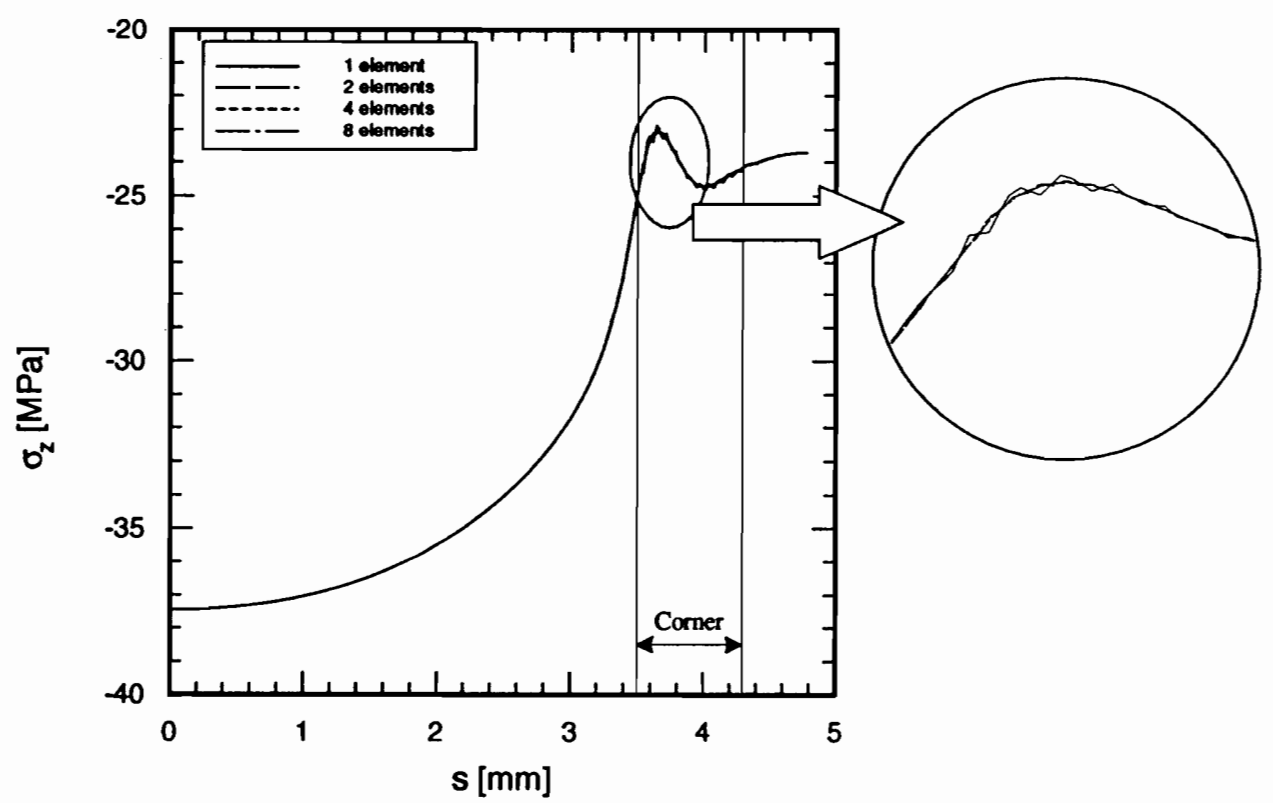

Figure 5.4: Convergence of axial stress $\sigma_{z}$ in $\mathrm{Si}_{3} \mathrm{~N}_{4}$ coating for a substrate with two $0.05 \mathrm{~mm}$ thick coatings $\left(w_{s u b}=4 \mathrm{~mm}, t_{s u b}=1 \mathrm{~mm}, r=0.5 \mathrm{~mm}\right)$. 


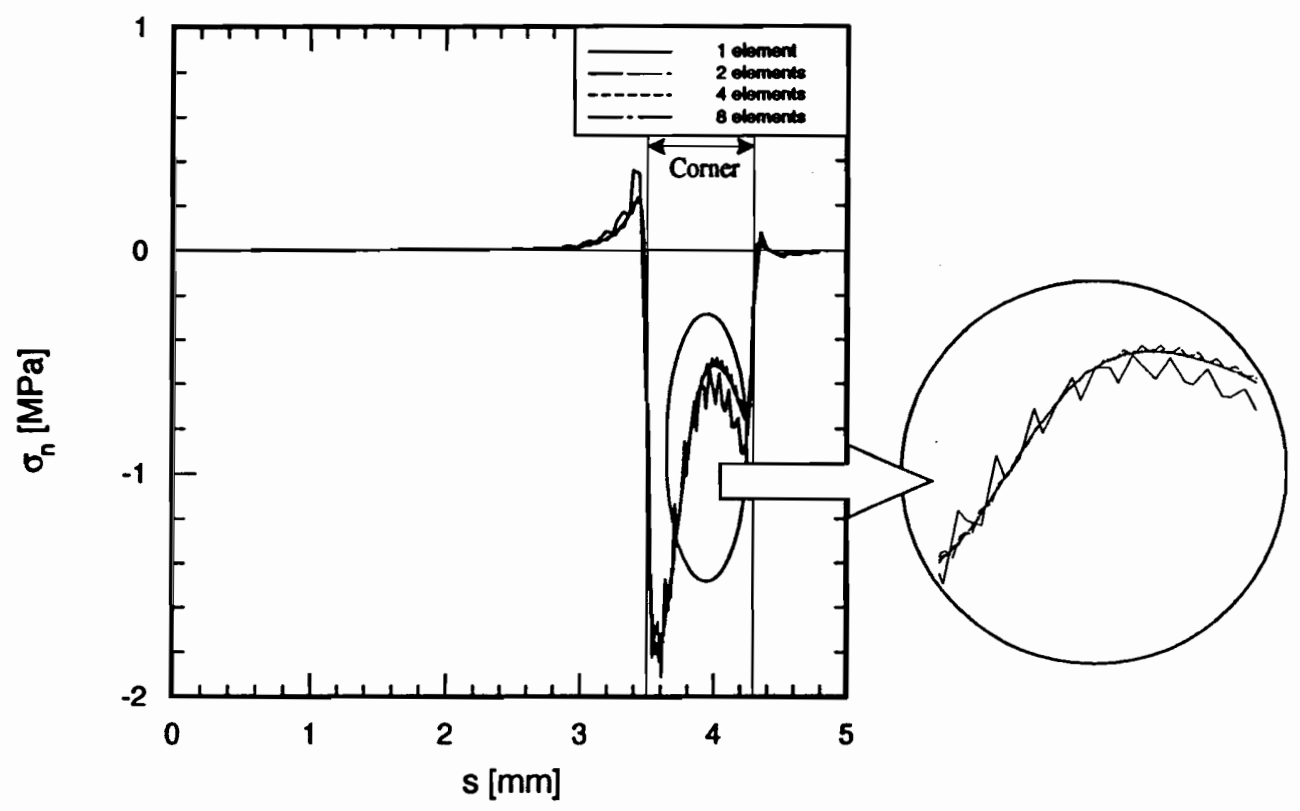

Figure 5.5: Convergence of normal stress $\sigma_{n}$ in $\mathrm{Si}_{3} \mathrm{~N}_{4}$ coating for a substrate with two $0.05 \mathrm{~mm}$ thick coatings $\left(w_{s u b}=4 \mathrm{~mm}, t_{s u b}=1 \mathrm{~mm}, r=0.5 \mathrm{~mm}\right)$.

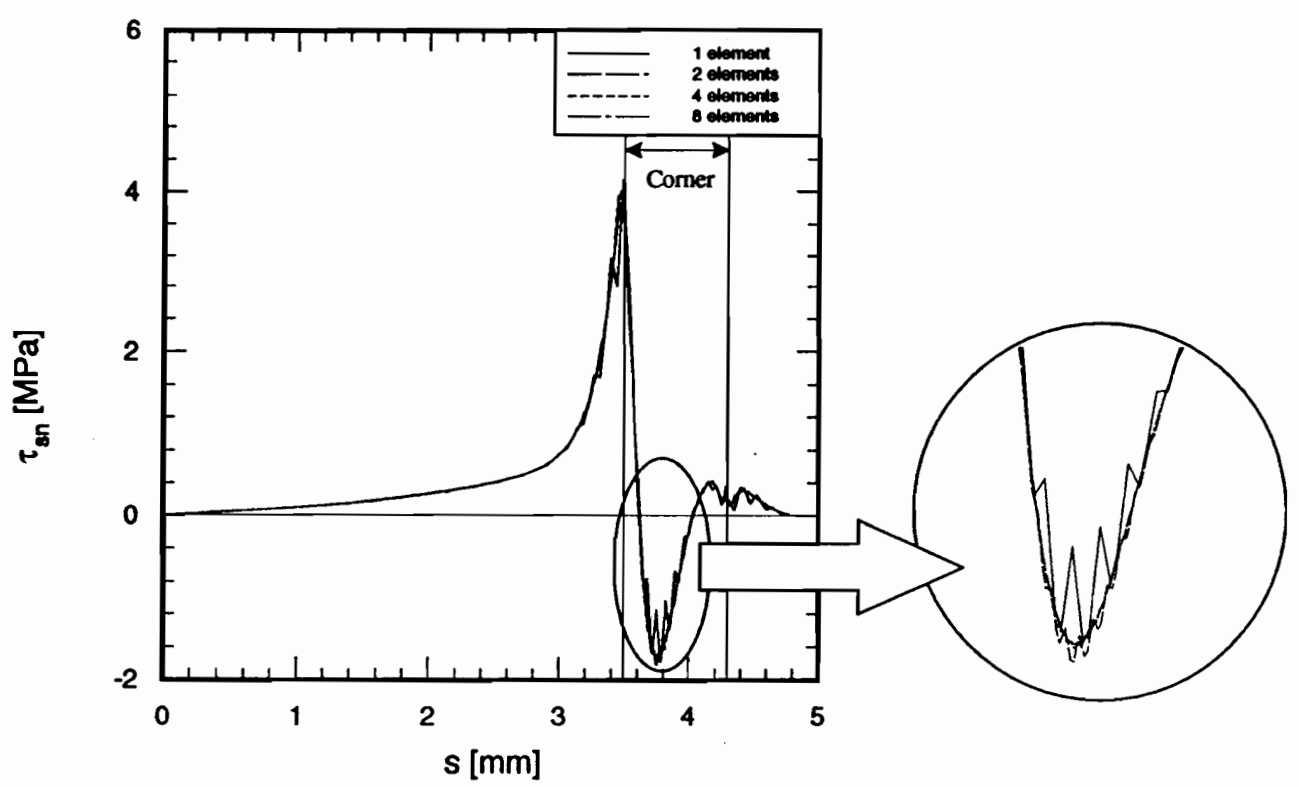

Figure 5.6: Convergence of shear stress $\tau_{s n}$ in $\mathrm{Si}_{3} \mathrm{~N}_{4}$ coating for a substrate with two $0.05 \mathrm{~mm}$ thick coatings $\left(w_{s u b}=4 \mathrm{~mm}, t_{s u b}=1 \mathrm{~mm}, r=0.5 \mathrm{~mm}\right)$. 


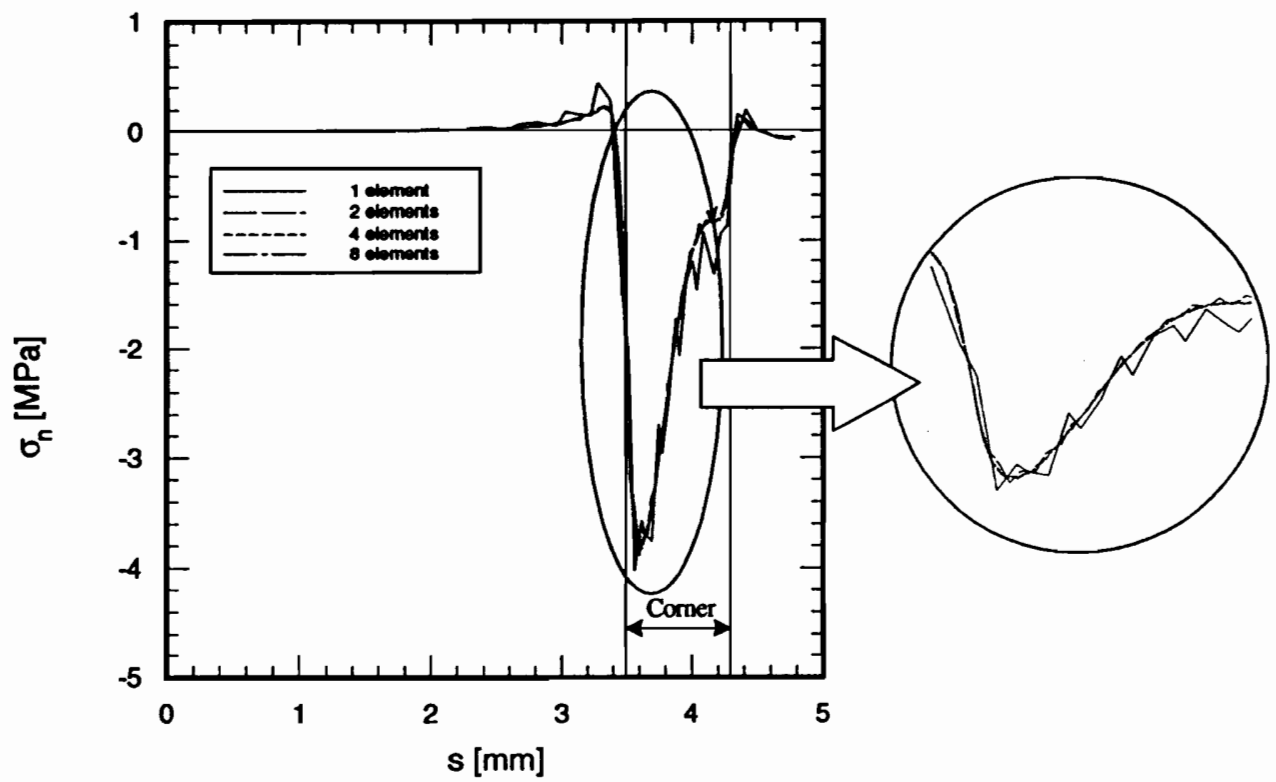

Figure 5.7: Convergence of normal stress $\sigma_{n}$ in $\mathrm{Si}_{3} \mathrm{~N}_{4}$ coating for a substrate with two $0.1 \mathrm{~mm}$ thick coatings $\left(w_{s u b}=4 \mathrm{~mm}, t_{s u b}=1 \mathrm{~mm}, r=0.5 \mathrm{~mm}\right)$.

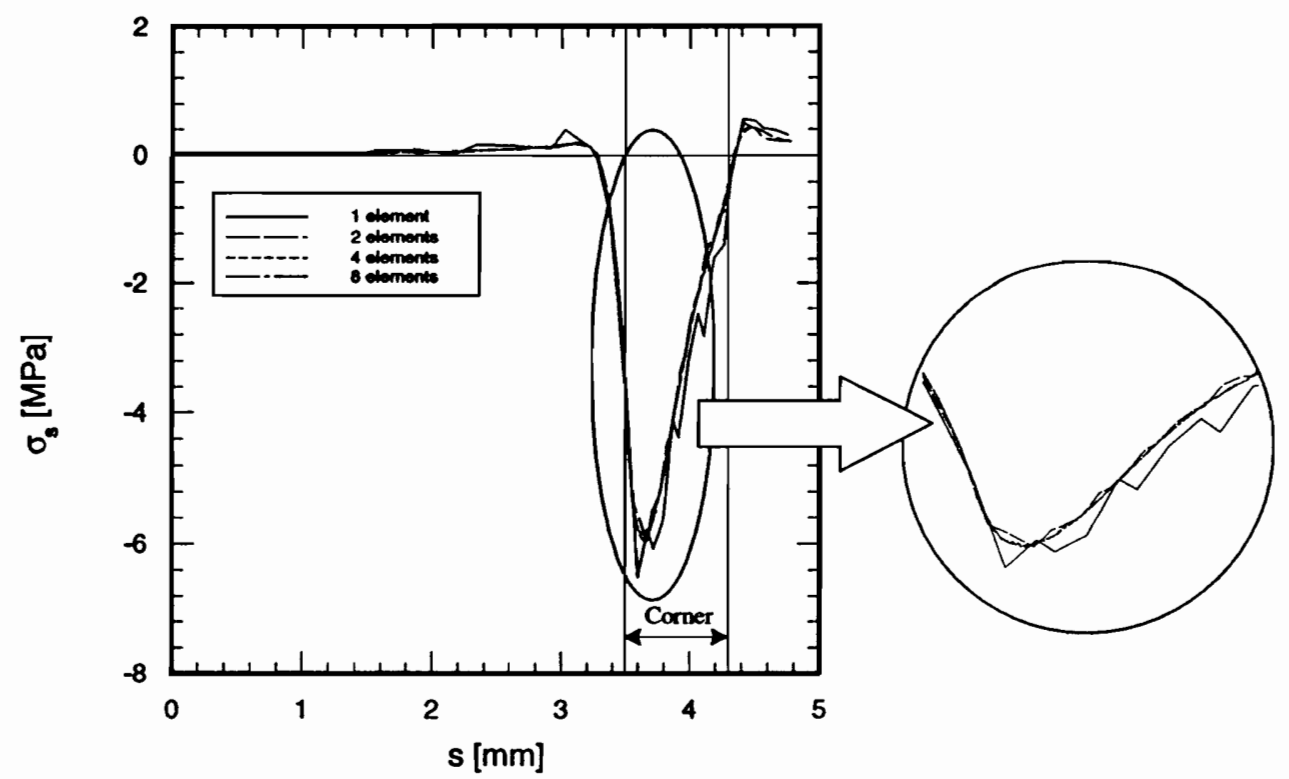

Figure 5.8: Convergence of normal stress $\sigma_{n}$ in $\mathrm{Si}_{3} \mathrm{~N}_{4}$ coating for a substrate with two $0.2 \mathrm{~mm}$ thick coatings $\left(w_{s u b}=4 \mathrm{~mm}, t_{s u b}=1 \mathrm{~mm}, r=0.5 \mathrm{~mm}\right)$. 
the thickness of a coating. As mentioned before, to reduce the influence of the transition elements on the stresses in the coatings and to prevent the oscillations, should they occur, from propagating into the coating, the finite element mesh is always generated so that four rows of quadrilateral elements are inserted between the inner coating and the transition elements in the substrate. From these observations the following conclusion can be drawn:

- The normal and shear stress components in the corner region are the critical stress components for the convergence.

- The thickness of the coatings does not significantly affect the convergence.

- For constant property coatings, 4 elements through the thickness of a coating give converged results.

- For coarse finite element meshes, disturbances generated by the transition from small to large elements in the substrate may propagate into the coatings. For finer meshes, this transition does not adversely affect stresses in the coatings or the substrate.

For geometries that are significantly different than the ones considered in this convergence study, more elements through the thickness of a coating may have to be used. Because the convergence depends primarily on the magnitude of the stress gradients, the above conclusions should also hold for temperature-dependent analyses with coatings applied at different temperatures. Though it will not be discussed, this was indeed the case for all temperature-dependent analysis with constant property coatings conducted in the course of this research.

\subsection{Influence of Corner Region on Stresses}

To understand the influence of the corner region on the stress field in the substrate and coatings away from the corner, a parametric study was conducted. As in the convergence study, $\mathrm{C} / \mathrm{C}$ substrates with $\mathrm{B}_{4} \mathrm{C}$ and $\mathrm{Si}_{3} \mathrm{~N}_{4}$ coatings applied at the same temperature and 


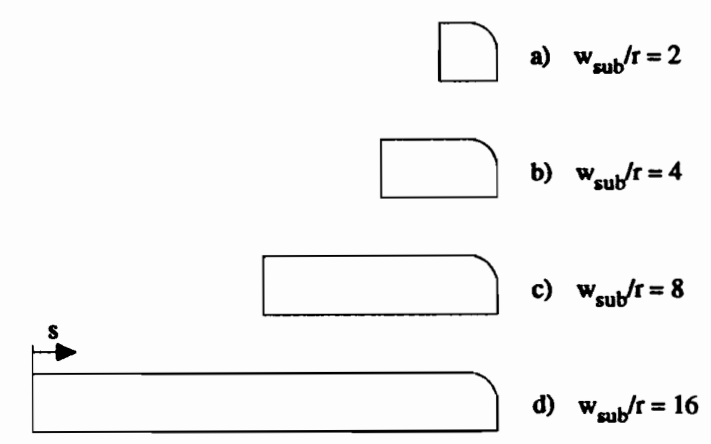

Figure 5.9: Geometry of models considered in the parametric study.

subjected to a $100^{\circ} \mathrm{C}$ temperature increase were analyzed. The width of the substrate, $w_{\text {sub }}$, was varied, while the substrate thickness, corner radius, and coating thicknesses were kept constant at $t_{s u b}=1 \mathrm{~mm}, r=0.5 \mathrm{~mm}$, and $t_{\text {layer }}=0.1 \mathrm{~mm}$, respectively. Four different width-to-radius ratios, $w_{\text {sub }} / r=2,4,8$, and 16 , were considered. What was being studied with the four width-to-radius ratios was the St. Venant effect. For anisotropic materials, the St. Venant effect involves both geometry and material properties. Ideally closed-form expressions for the region of influence could be developed, but here the problem was approached numerically. The geometries of the various width models are shown in Fig. 5.9. In the representation of the stresses, for smaller values of $w_{s u b} / r$, the $s$-coordinate is offset so that the corner regions of all the models map to the same $s$-coordinate. As an example, the stresses in the $\mathrm{B}_{4} \mathrm{C}$ coating for the different size models are compared in Fig. 5.10. In the discussion of the stresses, "case W2" refers to the analysis of the substrate and coating with $w_{s u b} / r=2$, "case W4" refers to the analysis of the substrate and coating with $w_{s u b} / r=4$, etc. The overall behavior of the stresses in the other coatings is similar to the one in the $\mathrm{B}_{4} \mathrm{C}$ coating. Hence the following discussion equally applies to those coatings.

For all stress components, the variation of the stresses with the $s$-coordinate is almost identical for cases W8 and W16. The stress components for cases W2 and W4 show a 
significant deviation from the stresses in cases W8 and W16. Away from the corner the shear stress $\tau_{s n}$ and the normal stress $\sigma_{n}$ should vanish. From Fig. 5.10 it is apparent that the normal stress is confined to the corner region for all cases. This stress component diminishes rapidly when moving away from the corner. The shear stress is also predominant in the corner region. It diminishes away from the corner, but interestingly, nowhere near as rapidly as the transverse normal stress. It appears that for case $\mathrm{W} 16, \tau_{s n}$ reaches its zero far-field value about 8 to 10 radii away from the corner. For the short cases, W2 and W4, $\tau_{s n}$ only approaches zero away from the corner because of the imposed symmetry boundary condition of the analysis. This shows that the corner has a significant effect on stresses in narrower substrates. For wide substrates the corner only effects the stress distribution locally, in the region of the corner.

In Table 5.2 the far-field stresses $(s=0)$ in the substrate and coatings calculated using the ISPSA are shown and compared to the far-field stresses calculated from the finite element analysis for the different widths substrates. Recall, the ISPSA stresses correspond to an infinitely wide composite without a corner. The stresses in composites with a finite width and a corner should approach these stresses away from the corner. As can be seen in Table 5.2, the far-field response for wide substrates is not effected by the corner and shows an excellent agreement with the stresses obtained using the ISPSA. For narrower substrates, the far-field stresses calculated using the finite element model are significantly larger than the stresses calculated using the ISPSA.

The influence of the material properties on the effect of the corner on the stresses was also investigated. This was done for the substrate and coating geometry with $w_{s u b} / r=16$ described in the preceding discussions. For this purpose the modulus of the inner coating, previously the $\mathrm{B}_{4} \mathrm{C}$ coating, was varied from $E=10 \mathrm{GPa}$ to $E=500 \mathrm{GPa}$. The shear modulus was varied accordingly. The other properties for the inner coating were kept at the values indicated by $\mathbf{a}^{*}$ in Table A.5. The four stress components $\sigma_{s}, \sigma_{z}, \sigma_{n}$, and $\tau_{s n}$ are shown in Fig. 5.12 for the altered inner coating and in Fig. 5.13 for the $\mathrm{Si}_{3} \mathrm{~N}_{4}$ coating. Recall, both coatings were applied at the same temperature and the temperature 

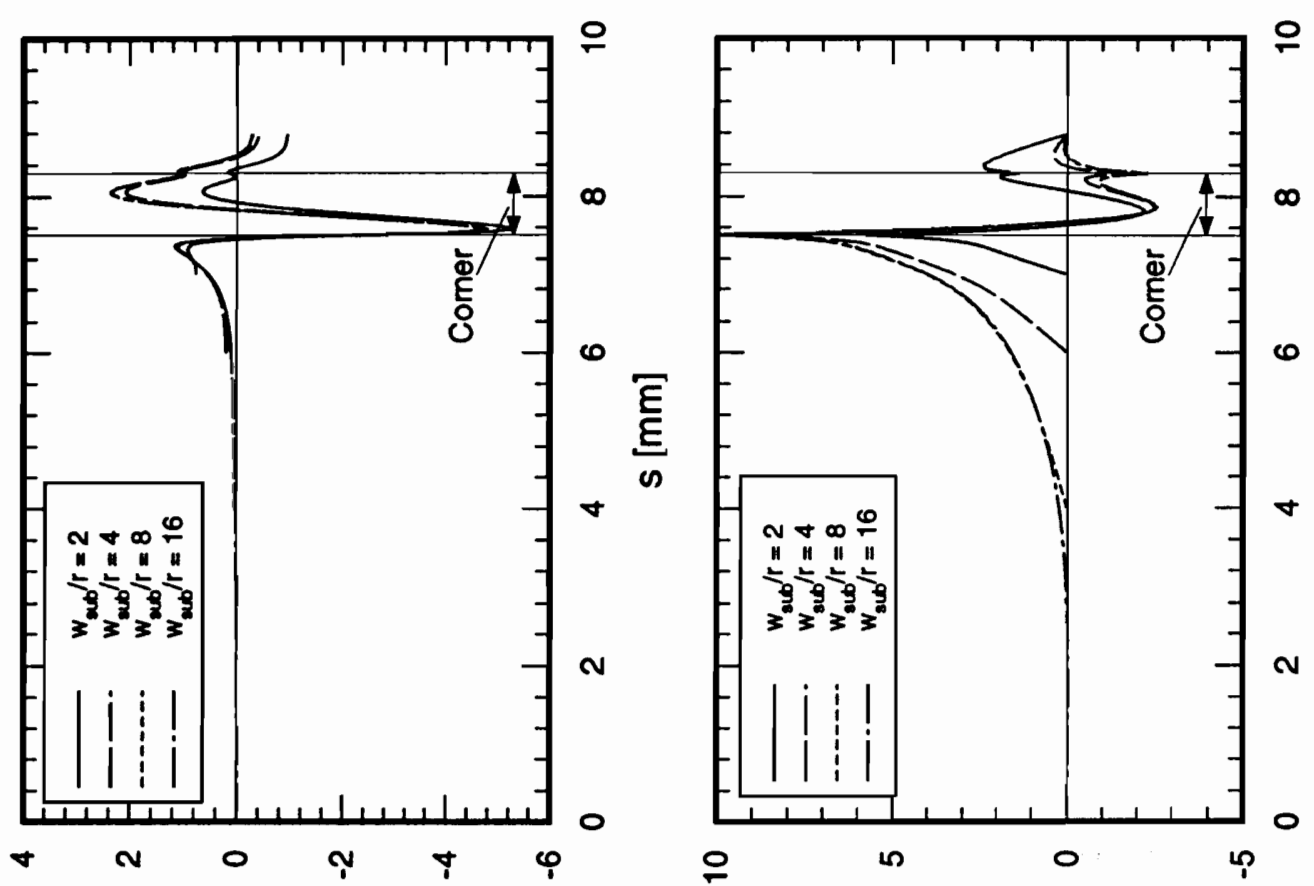

으

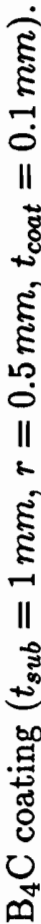

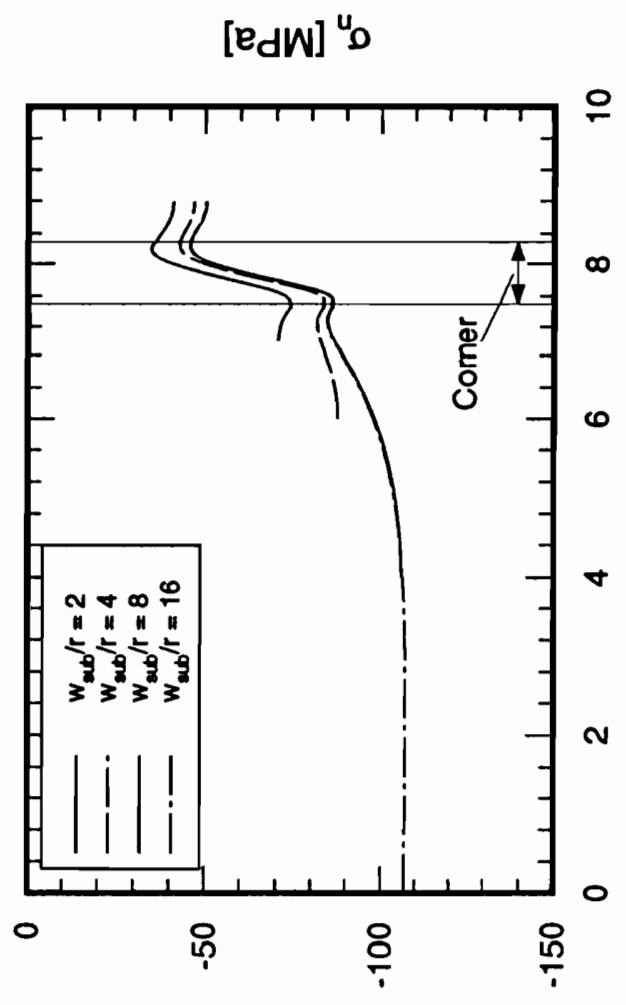

$\left[e_{d W}\right]^{s}$

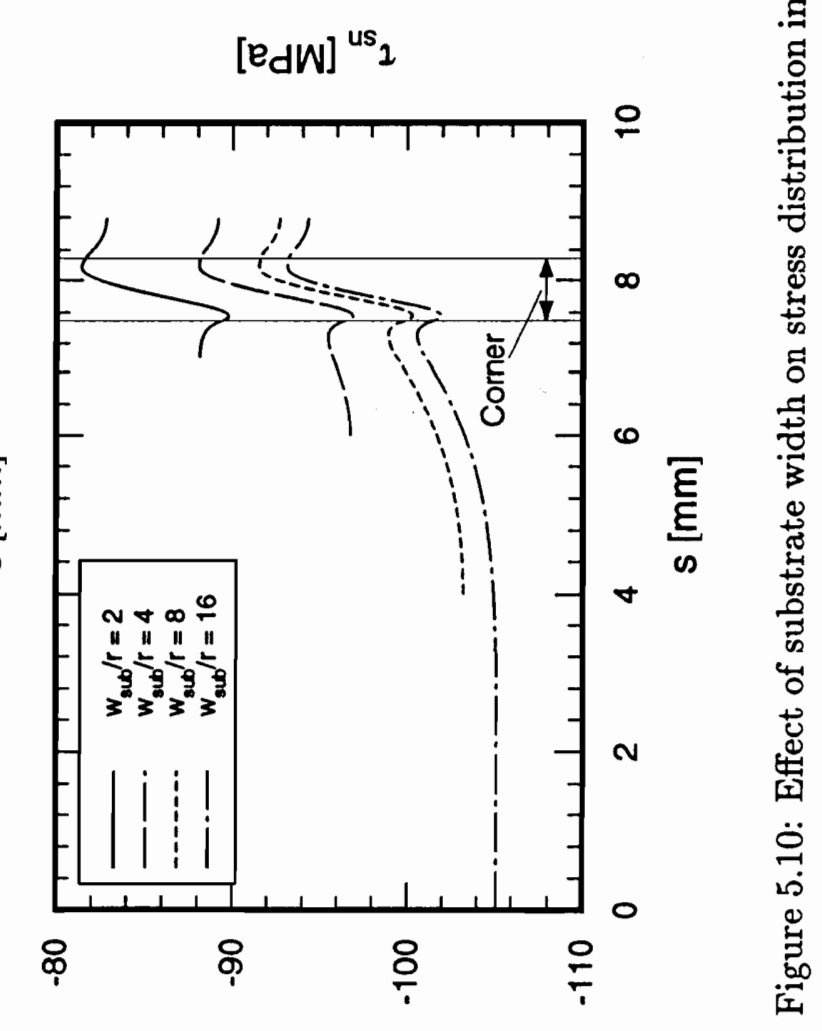

$\left[e_{d W}\right]_{0}$ 

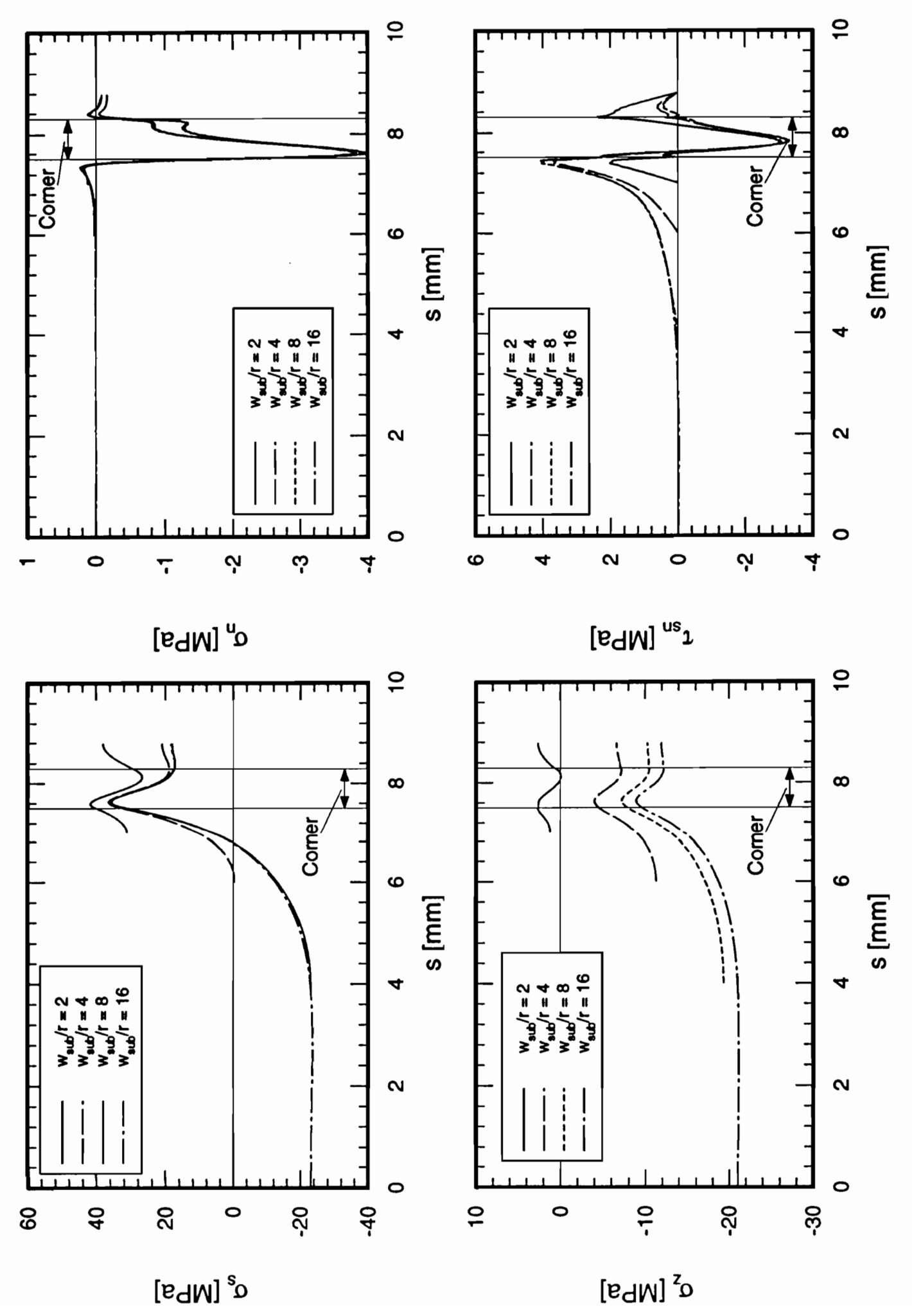

$\left[e_{\mathrm{dW}}\right]^{\mathrm{s}} \mathrm{o}$

$\left[\mathrm{C}^{\mathrm{d} W}\right]^{\mathrm{z}}$ 
Table 5.2: Comparison of far-field stresses calculated using the finite element model for different width models and stresses calculated using ISPSA.

\begin{tabular}{|c|c|c|c|c|}
\hline & Case & Substrate & $\mathrm{B}_{4} \mathrm{C}$ & $\mathrm{Si}_{3} \mathrm{~N}_{4}$ \\
\hline & W2 & 18.94 & -70.77 & 31.12 \\
& W4 & 17.78 & -87.54 & -0.4 \\
$\sigma_{s}[\mathrm{MPa}]$ & W8 & 13.31 & -106.0 & -23.11 \\
& W16 & 12.85 & -107.1 & -23.11 \\
& ISPSA & 12.97 & -106.8 & -22.85 \\
\hline & W2 & 17.68 & -87.90 & 1.19 \\
& W4 & 15.60 & -96.71 & -11.31 \\
$\sigma_{z}[\mathrm{MPa}]$ & W8 & 14.21 & -103.2 & -19.32 \\
& W16 & 13.58 & -105.8 & -21.05 \\
& ISPSA & 12.97 & -106.8 & -22.85 \\
\hline
\end{tabular}

was increased by $100^{\circ} \mathrm{C}$. The stresses at the midthickness location of each coating are shown. First consider the tangential stress $\sigma_{s}$ in the altered inner coating. It can be seen that, as expected, the stress significantly depends on the modulus of the coating. Because for the lower moduli cases the stress is lower away from the corner, the stress in the corner region is lower, too. Similarly, the axial stress $\sigma_{z}$ also depends significantly on the modulus. As shown in Fig. 5.13, the trend is reversed in the $\mathrm{Si}_{3} \mathrm{~N}_{4}$ coating. For a lower modulus in the inner coating, the tangential and axial stresses are higher in the $\mathrm{Si}_{3} \mathrm{~N}_{4}$ coating. If the modulus of the inner coating is reduced, then the average stiffness of all coatings combined is reduced, and therefore the substrate can more freely deform. This added deformation of the substrate causes the $\mathrm{Si}_{3} \mathrm{~N}_{4}$ coating to strain more and thus generates higher stresses in that coating. However, the stress concentrations in the $\mathrm{Si}_{3} \mathrm{~N}_{4}$ coating for the components $\sigma_{s}$ and $\sigma_{z}$ at the beginning of the corner are greatly reduced for lower moduli of the inner coating. In fact, for higher moduli inner coatings, the stress concentration in the $\mathrm{Si}_{3} \mathrm{~N}_{4}$ coating is such that $\sigma_{s}$ becomes positive in the corner.

The shear stress and the normal stress for both coatings are confined to the corner region for all moduli of the inner coating. Details of these stresses are shown in Figs. 5.14 - 5.17. The region along the $s$-coordinate over which these stresses are present appears to be in- 

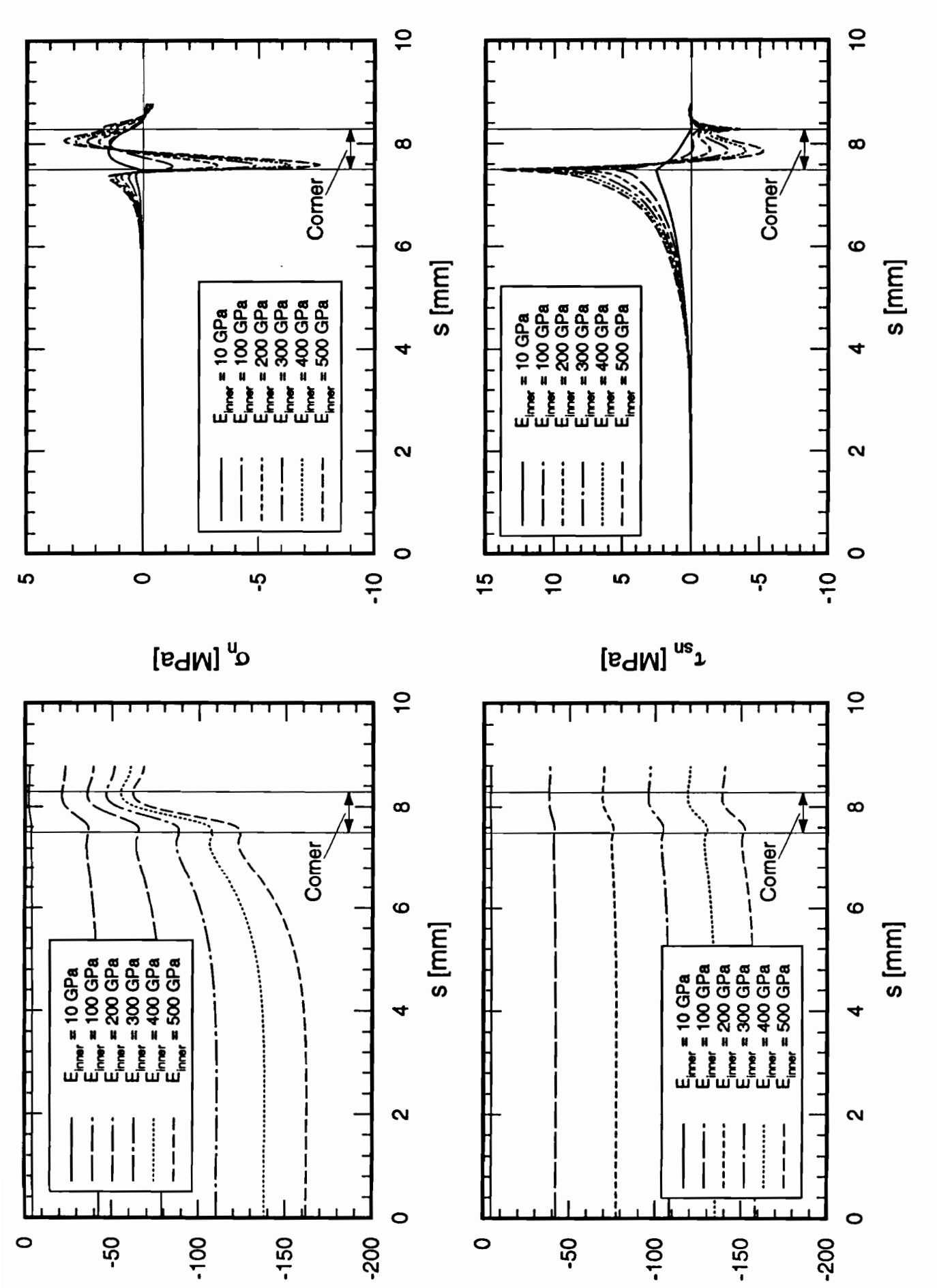

$\left[e_{d W}\right]^{s}$

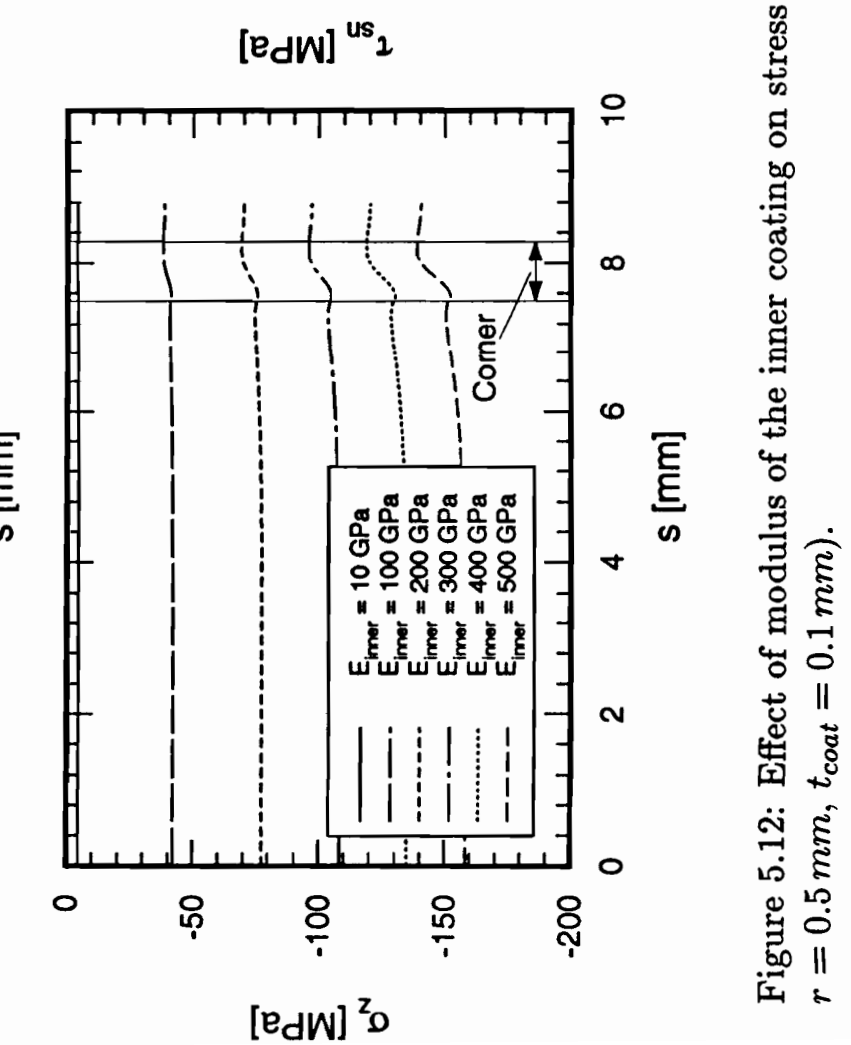



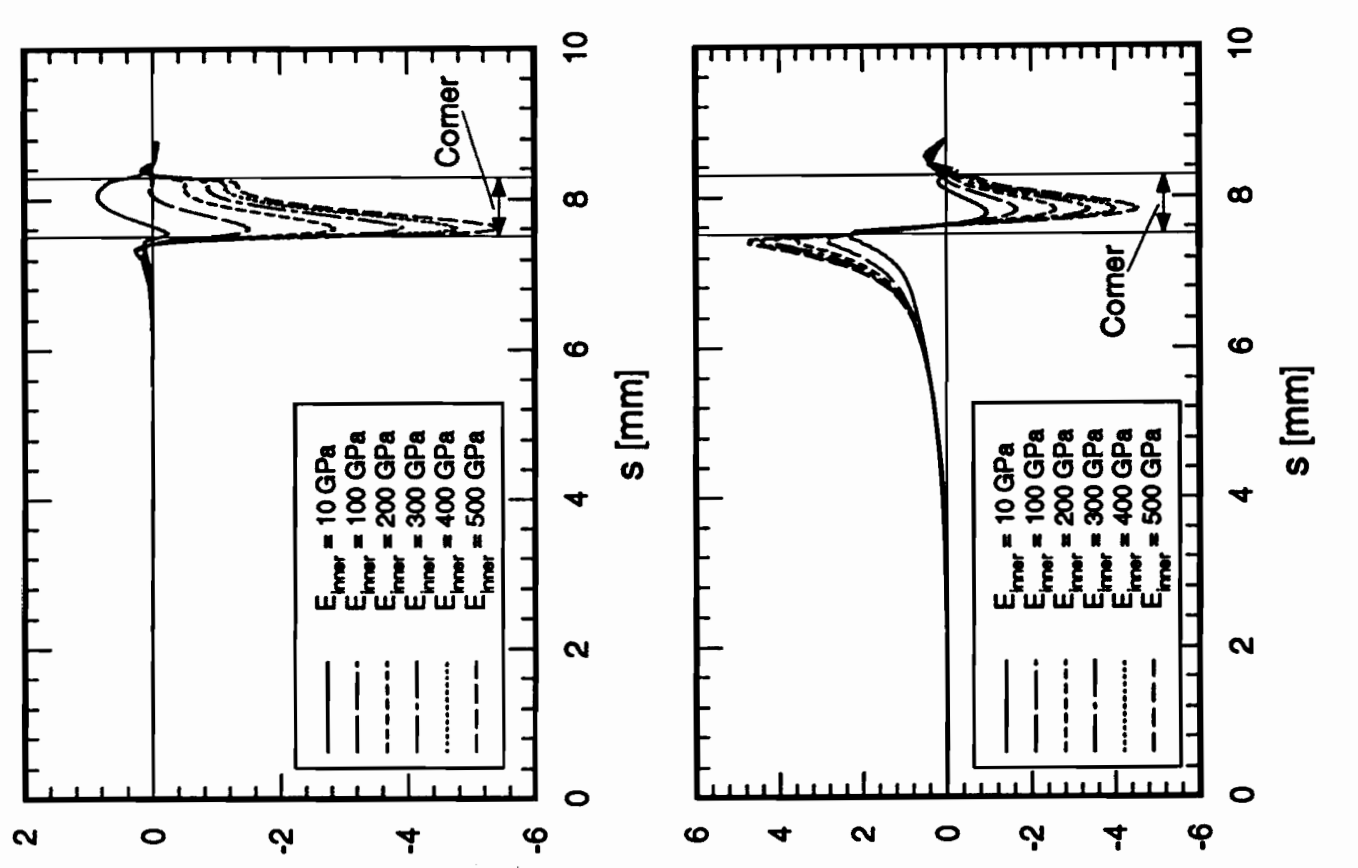

है
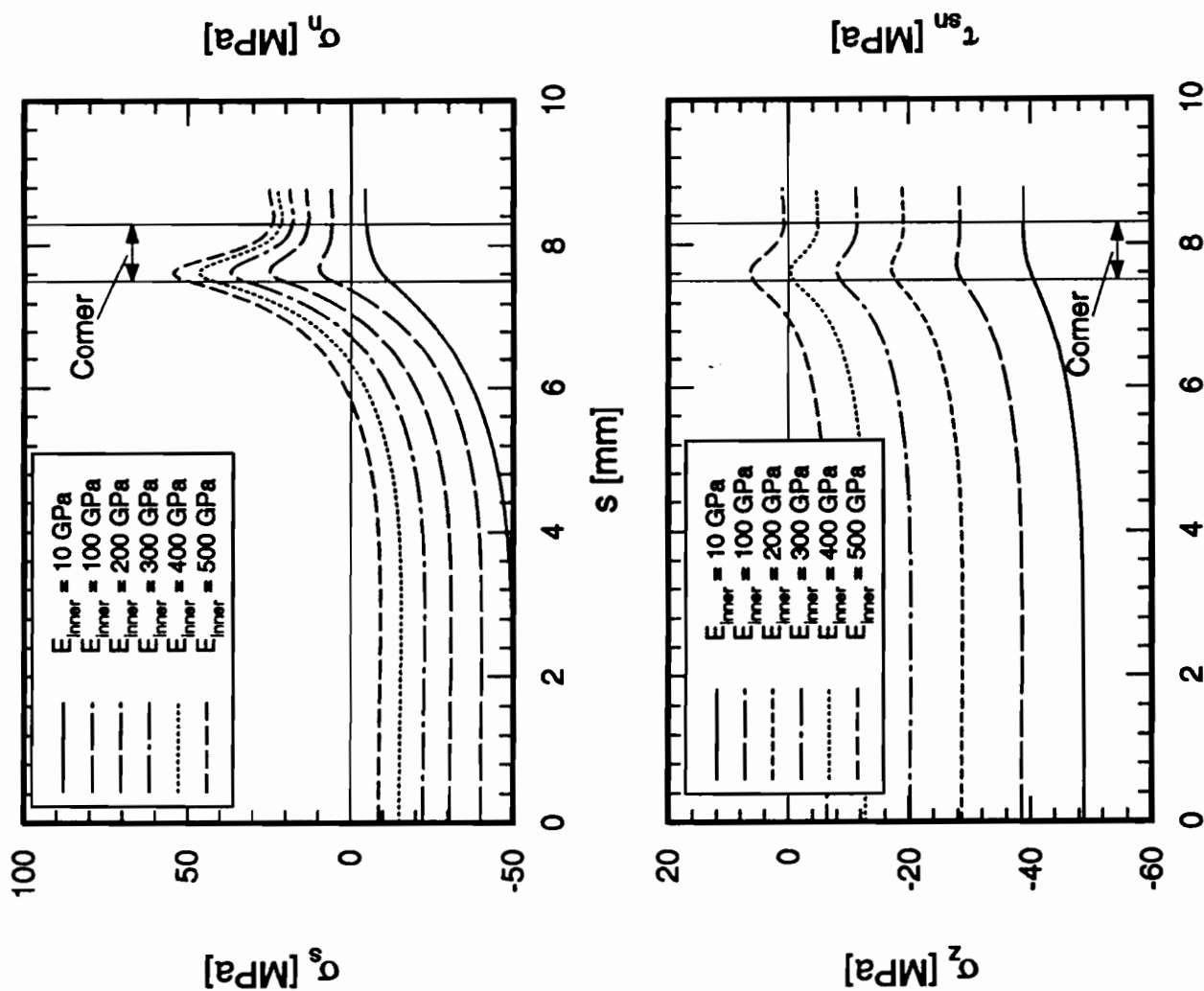

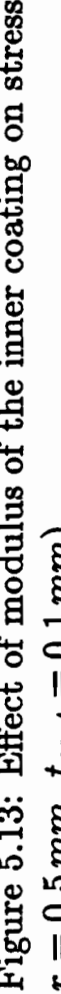

$\left[e_{d W}{ }^{s}\right.$

$\left[e_{d W}\right]^{2}$ 
dependent of the modulus of the inner coating. As can be seen in Figs. 5.14 and 5.16, the magnitude of the normal stress in both coatings depends significantly on the modulus of the inner coating. The normal stress decreases for a lower modulus inner coating. The stress concentrations in the shear stress in the inner coating, Fig. 5.15, are also reduced significantly for a lower modulus inner coating. For the shear stress in the $\mathrm{Si}_{3} \mathrm{~N}_{4}$ coating, Fig. 5.17, the stress concentration at the beginning of the corner does not change with the modulus of the inner coating. However, the stress concentration at the end of the corner decreases with decreasing modulus. The lower modulus inner coating minimizes the stress concentration effect in the $\mathrm{Si}_{3} \mathrm{~N}_{4}$.

In summary:

- For large width-to-radius ratios the corner does not influence the stresses away from the corner. Also, stresses in the corner region do not change with increasing substrate width for widths larger than some critical width-to-radius ratios. This ratio is approximately $w_{s u b} / r=10$.

- For small width-to-radius ratios the corners interact. Stresses in the corner region and "away" from the corner are influenced by the corner.

- Shear and normal stresses are predominant in the corner region. While the normal stress diminishes rapidly away from the corner, the shear stress diminishes only slowly.

- Large stress concentrations in the tangential stress are present at the beginning of the corner.

- The ISPSA accurately predicts far-field stresses for width-to-radius ratios larger than the critical width-to-radius ratio.

- Though the modulus of elasticity of the inner coating significantly influences the magnitude of the stresses, it does not affect the region of influence of the corner. In other words, the critical width-to-radius ratio is independent of the modulus of the inner coating. 


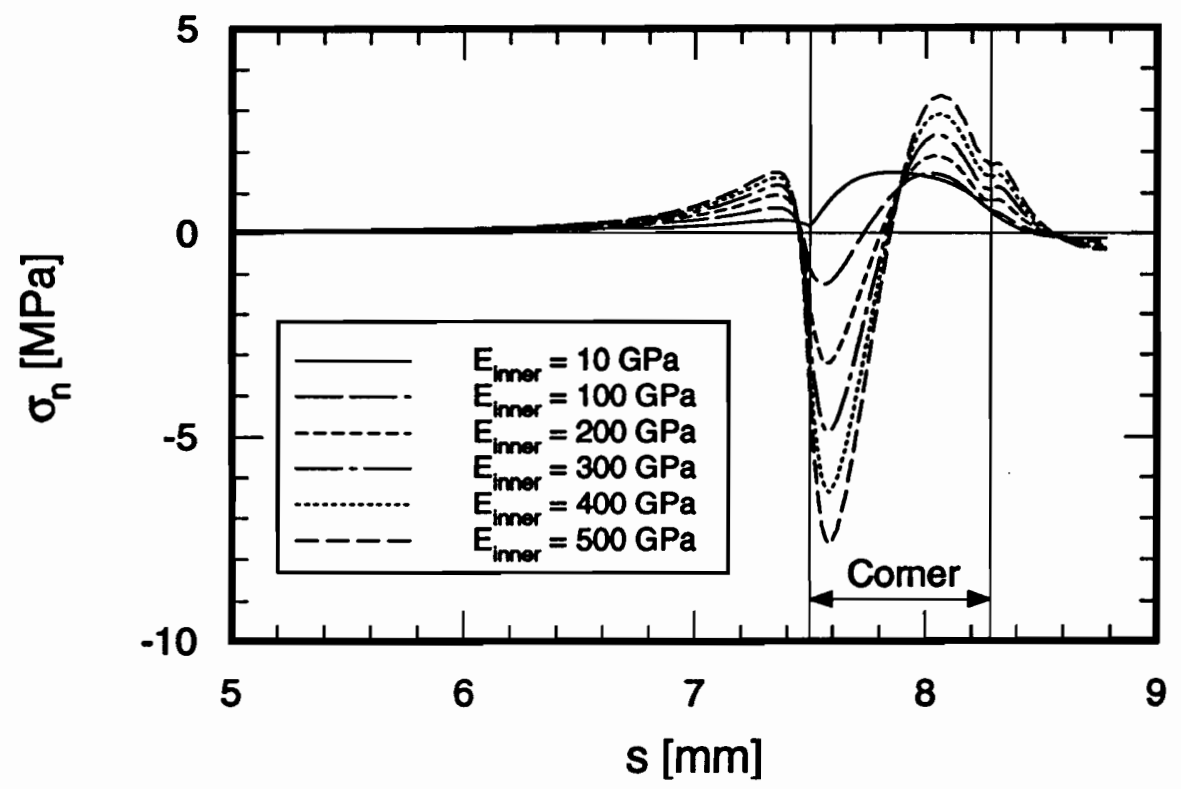

Figure 5.14: Details of normal stress $\sigma_{n}$ in inner coating for different moduli of the inner coating $\left(w_{s u b}=8 \mathrm{~mm}, t_{s u b}=1 \mathrm{~mm}, r=0.5 \mathrm{~mm}, t_{\text {coat }}=0.1 \mathrm{~mm}\right)$.

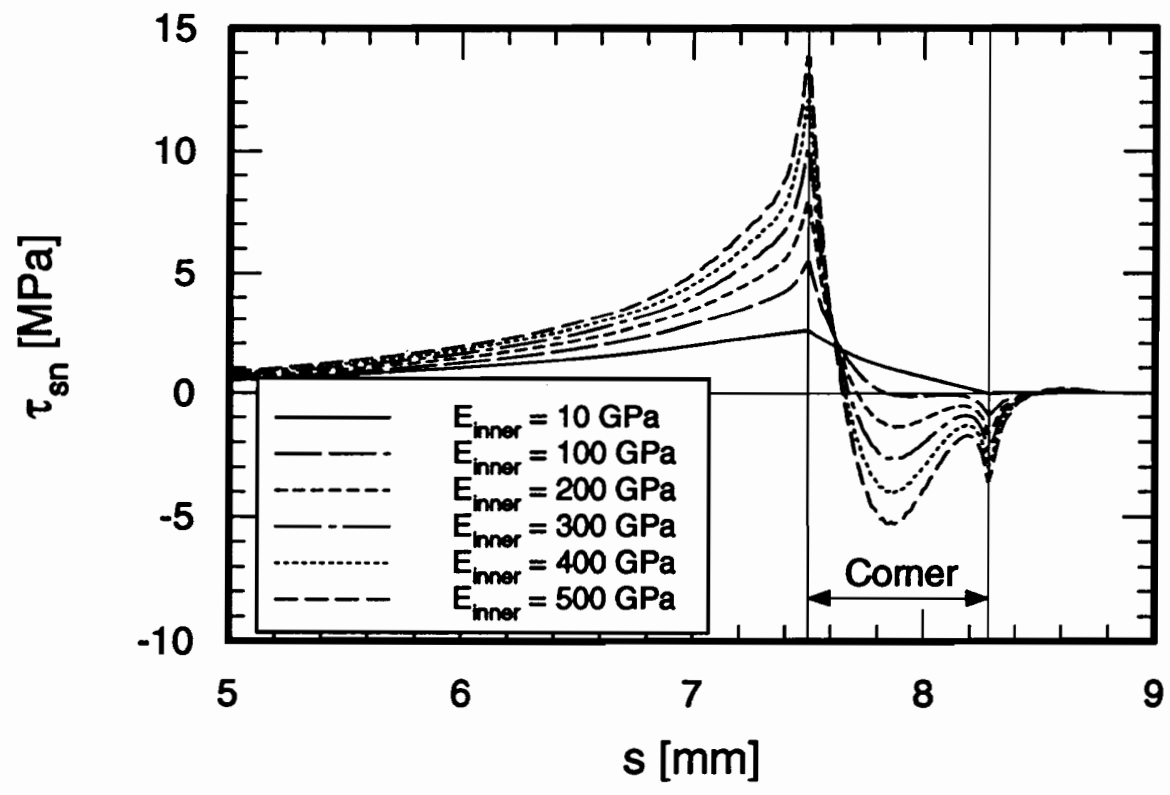

Figure 5.15: Details of shear stress $\tau_{s n}$ in inner coating for different moduli of the inner coating $\left(w_{s u b}=8 \mathrm{~mm}, t_{s u b}=1 \mathrm{~mm}, r=0.5 \mathrm{~mm}, t_{\text {coat }}=0.1 \mathrm{~mm}\right)$. 


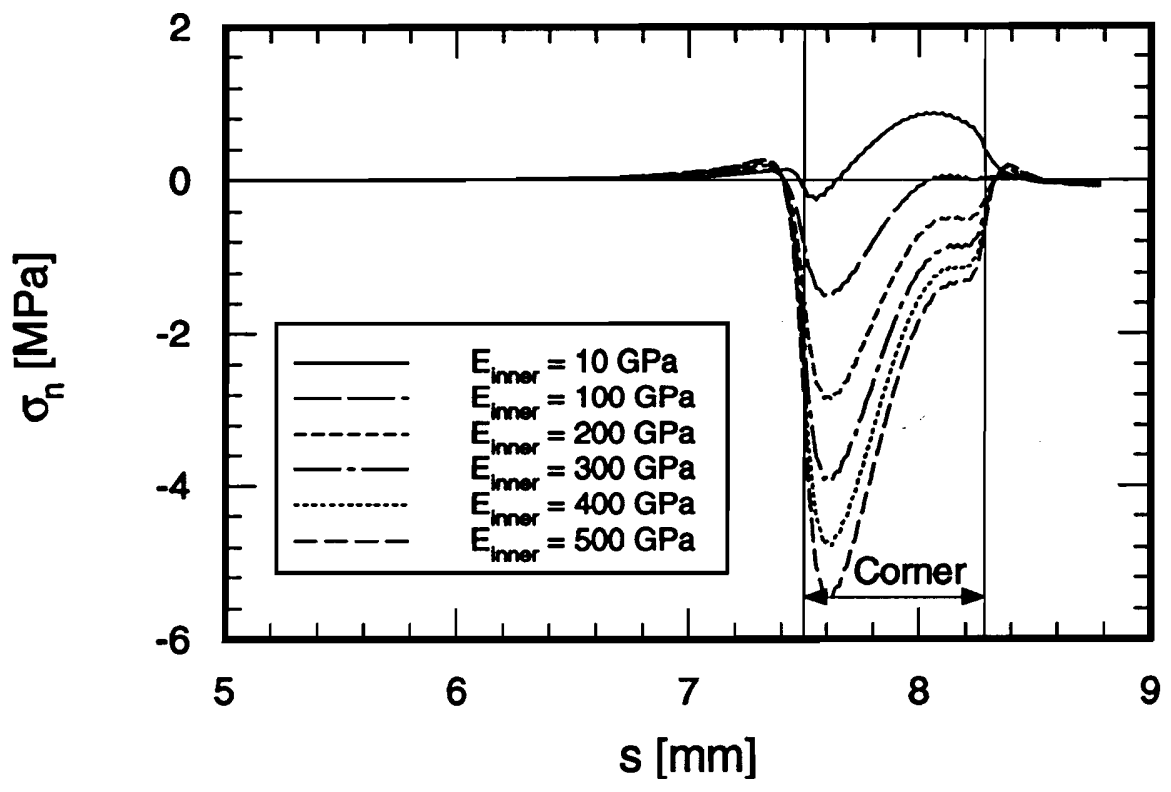

Figure 5.16: Details of normal stress $\sigma_{n}$ in $\mathrm{Si}_{3} \mathrm{~N}_{4}$ coating for different moduli of the inner coating $\left(w_{s u b}=8 \mathrm{~mm}, t_{s u b}=1 \mathrm{~mm}, r=0.5 \mathrm{~mm}, t_{\text {coat }}=0.1 \mathrm{~mm}\right)$.

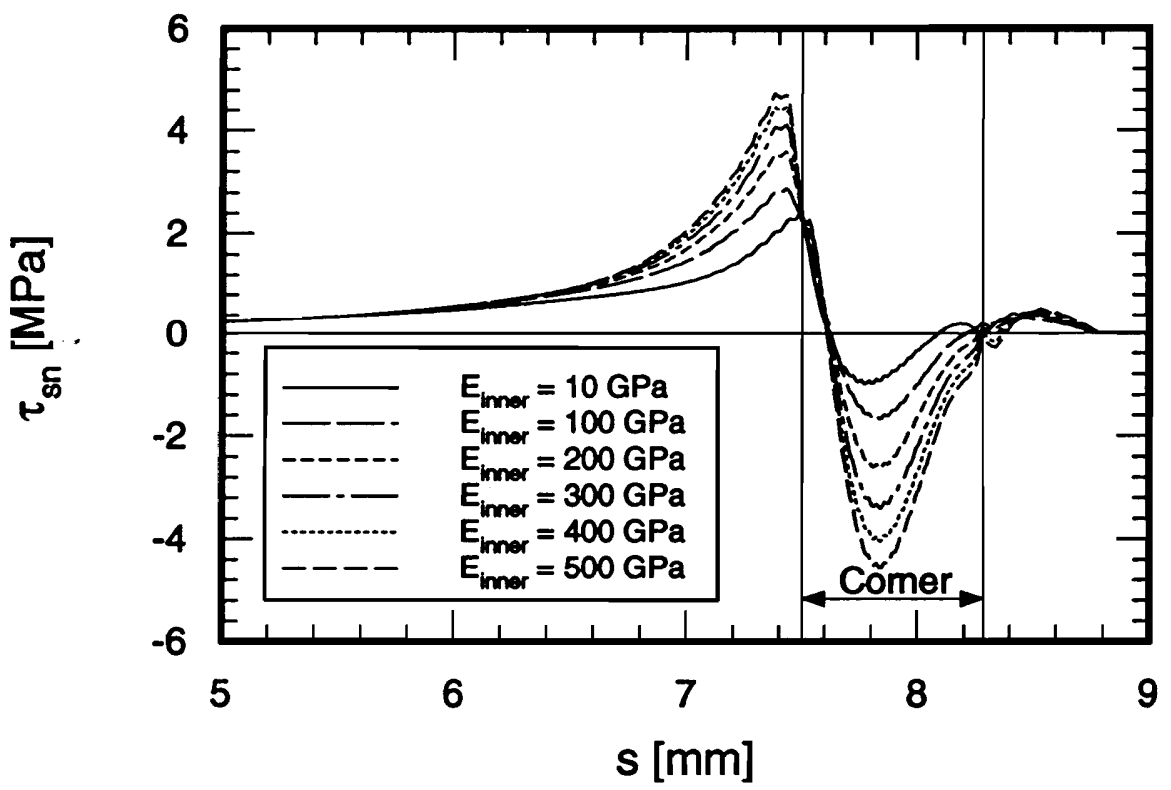

Figure 5.17: Details of shear stress $\tau_{s n}$ in $\mathrm{Si}_{3} \mathrm{~N}_{4}$ coating for different moduli of the inner coating $\left(w_{s u b}=8 \mathrm{~mm}, t_{s u b}=1 \mathrm{~mm}, r=0.5 \mathrm{~mm}, t_{\text {coat }}=0.1 \mathrm{~mm}\right)$. 
Several conclusions relating to the modeling of substrates with coatings can be drawn from this study:

- For narrow substrates for which the corners interact, the entire composite needs to be modeled using finite elements to obtain accurate stresses.

- For wide substrates only the region up to the critical width-to-radius ratio may needed to be modeled using finite elements. Far-field stresses can be predicted using a simplified analysis like the ISPSA. The ISPSA stresses could be used as boundary conditions on the reduced size finite element model.

The geometries considered in the present study allowed for modeling of the entire composite with finite elements. Thus, the reduced size modeling approach was not used. However, for geometries on the extremes of the dimensions indicated on page 16 , it would not be possible to model the entire composite using finite elements and the reduced size modeling approach would have to be used.

\subsection{Influence of Substrate Orthotropy}

As the tangential coordinate $s$ increases from values "before the corner" to values "after the corner", it is clear that the changing geometry due to the corner influences the character of the various stress components. This was observed in the figures in the previous section. There is, however, another important influence on the character of the stresses in the corner region. The variation of the material properties of the substrate due to the rotation of the $s-n-z$ coordinate system when moving in the direction of $s$ around the corner contributes significantly to the variation of the stresses. The rotation of the s-n-z coordinate system in the corner region was illustrated in Fig. 5.1. To demonstrate this, the orthotropic substrate in the above study was replaced by two different isotropic substrates for the case with $w_{s u b} / r=16$. For the first isotropic case, the stiff inplane properties of the orthotropic substrate were used as the isotropic properties, i.e., the substrate properties were $E=$ 
$100.0 \mathrm{GPa}, \nu=0.07$, and $\alpha=0.1 \times 10^{-6} /{ }^{\circ} \mathrm{C}$. For the second isotropic case, the soft transverse properties were used as the isotropic properties, i.e., the substrate properties were $E=5.0 \mathrm{GPa}, \nu=0.2$, and $\alpha=4.8 \times 10^{-6} /{ }^{\circ} \mathrm{C}$. The properties of the coatings were kept the same for all cases. As in the previous section, both coatings were applied at the same temperature, and the composite was subjected to a $100^{\circ} \mathrm{C}$ temperature increase. The stresses at the midthickness location of the $\mathrm{B}_{4} \mathrm{C}$ coating for the cases with the orthotropic substrate and the two isotropic substrates are compared in Fig. 5.18. In the legend of the figure, the case with the actual orthotropic substrate is denoted as "orthotropic", the case with the hypothetical isotropic substrate with the stiff properties is denoted as "isotropic, stiff", and the case with the hypothetical isotropic substrate with the soft properties is denoted as "isotropic, soft".

Consider the tangential stress $\sigma_{s}$ in Fig. 5.18. For the composite with the orthotropic substrate, a large variation of $\sigma_{s}$ with $s$ can be observed in the corner region. In contrast, only small variations are present for the composites with isotropic substrates. The variation of stress in composites with isotropic substrates can be attributed solely to the geometric discontinuity. However, in addition to the geometric discontinuity, the variation of the material properties of the orthotropic substrate due to the rotation of the s-n-z coordinate system when moving in the direction of $s$ around the corner contributes significantly to the variation of the stresses. For values of $s$ before the corner, the orthotropic substrate has a high modulus and a very low coefficient of thermal expansion in the $s$ direction. In this region the coefficient of thermal expansion of the substrate is much lower than the coefficient of thermal expansion of the coatings. Hence, large stresses are generated in the coatings before the corner. As can be seen in Fig. 5.18, these stresses are close to the stresses generated for the case with the isotropic substrate with stiff material properties. For values of $s$ after the corner, the modulus of the orthotropic substrate in the $s$ direction is very low, while the coefficient of thermal expansion now is larger than the coefficient of thermal expansion of either coating. Hence, only low stresses are generated in the coatings after the corner. These stresses are close to the stresses for the case with the isotropic substrate with 

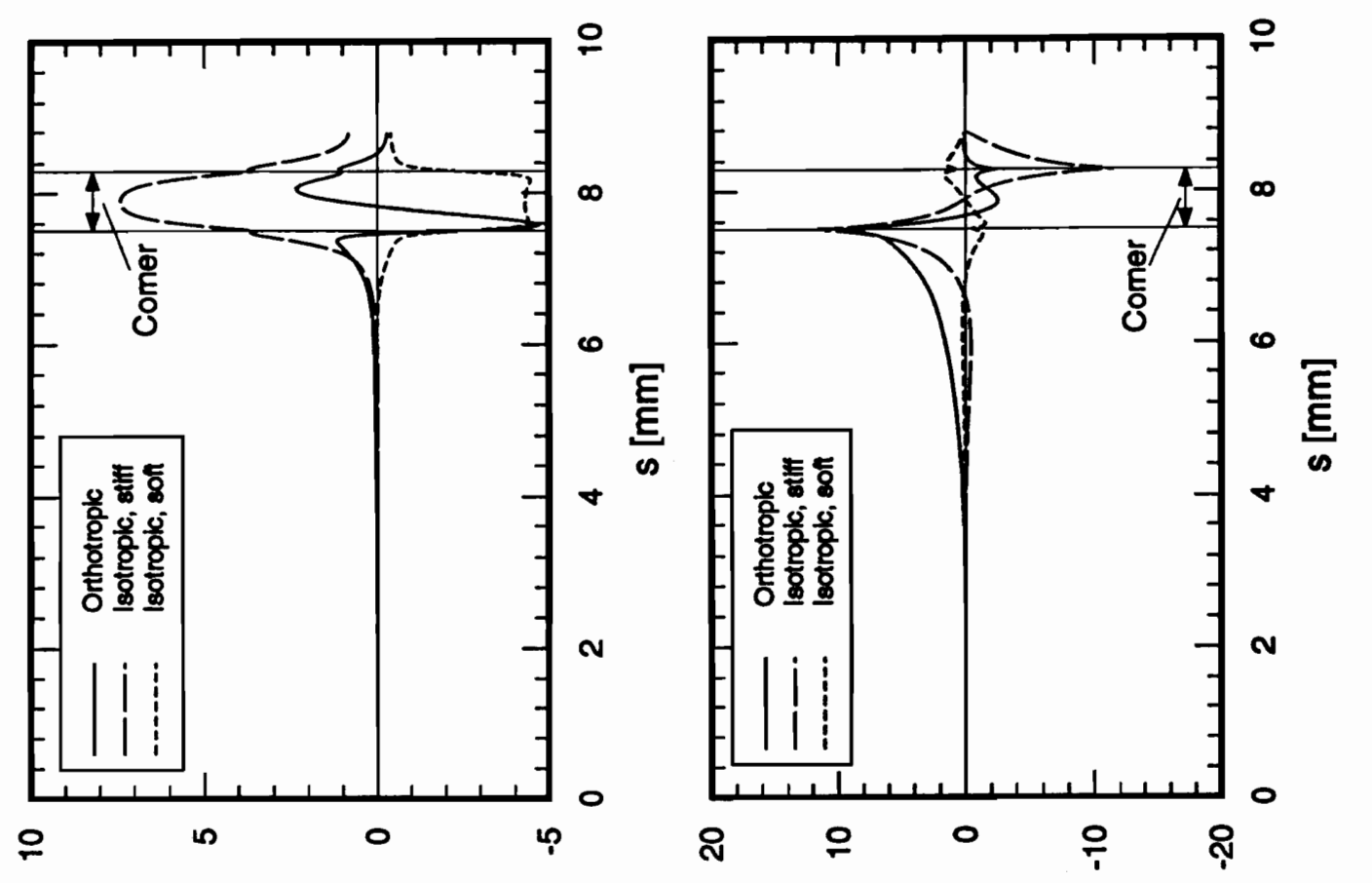

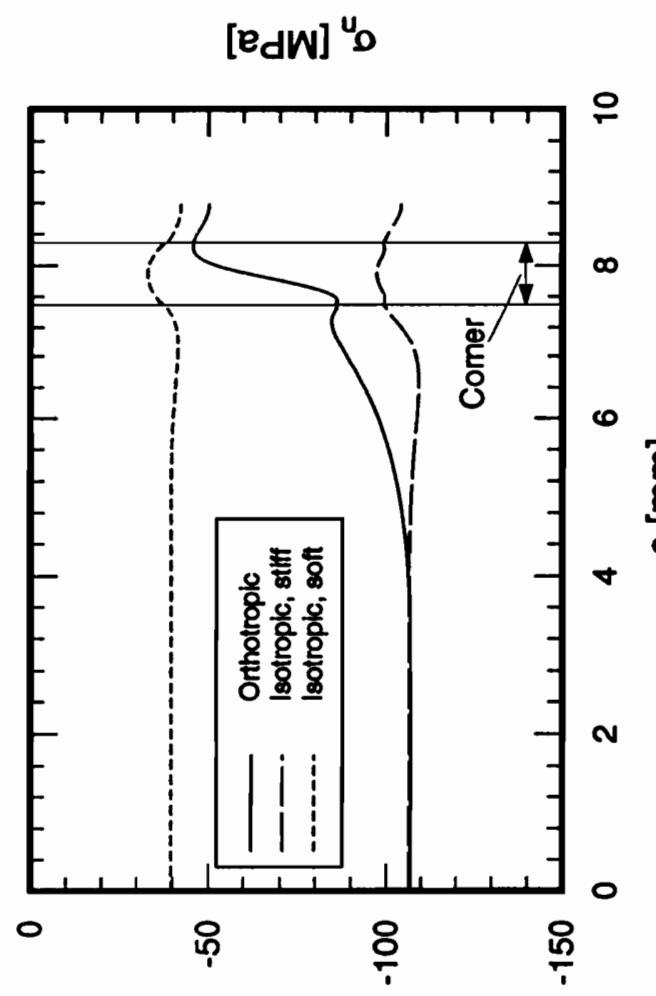

[edW] ${ }^{s}$

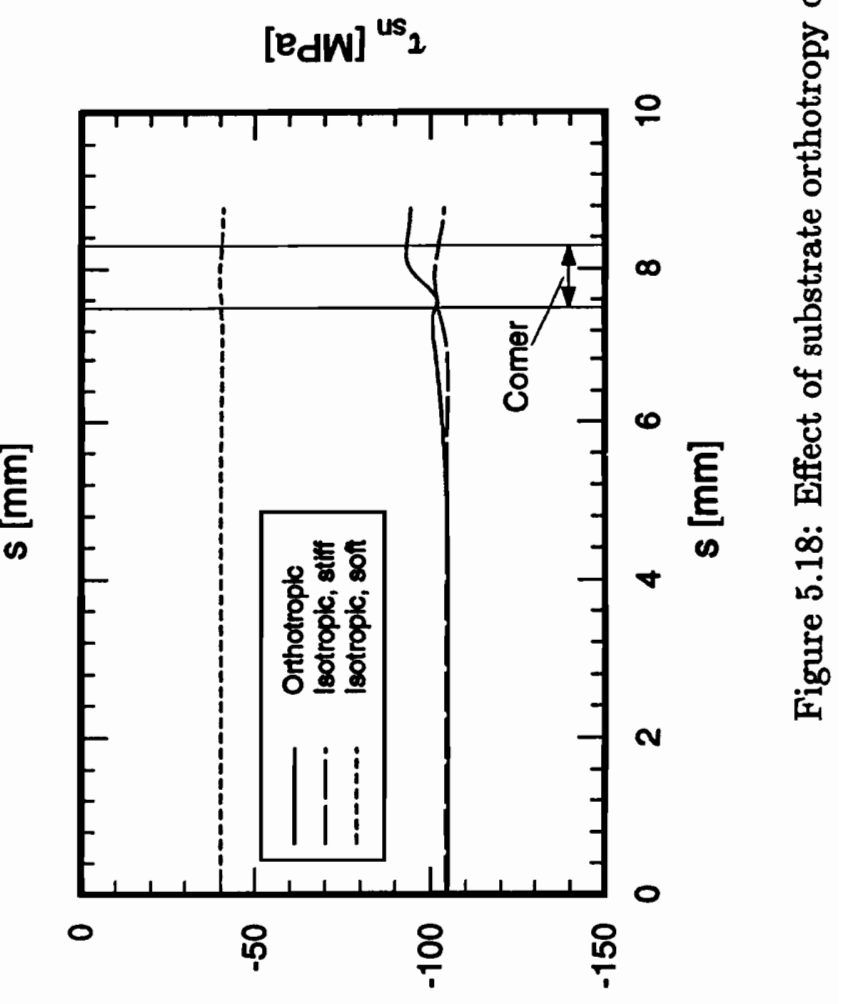

[edw] ${ }^{z}$ 
soft properties. Thus, the large variation of the stress component $\sigma_{s}$ with $s$ in the corner region of the composite with the orthotropic substrate can be attributed to the geometric discontinuity and to the variation of the material properties. Also, notice in the character of $\sigma_{s}$ that the region of influence of the corner region is increased for the composite with the orthotropic substrate. The variation of stress begins at lower values of $s$ for the composite with the orthotropic substrate than for the composites with either isotropic substrate.

In contrast to the tangential stress $\sigma_{s}$, for the axial stress $\sigma_{z}$, the orthotropy of the substrate only causes small changes in the corner region. The variation of the axial stress in the corner region is due to its Poisson coupling to the other stress components. Because the properties of the orthotropic substrate and the stiff isotropic substrate are the same in the axial direction, the magnitudes of the axial stresses for these two cases are close. The much lower axial modulus of the soft isotropic substrate results in much lower axial stresses compared to the stiff isotropic and the orthotropic case.

The orthotropy of the substrate also has a significant influence on the normal stress, $\sigma_{n}$, and the shear stress, $\tau_{s n}$. The normal stress shows a smooth variation in the corner region for the isotropic substrates. It is symmetric with respect to the center of the corner. For the stiff isotropic substrate the normal stress is tensile throughout the corner region, and for the soft isotropic substrate it is compressive throughout the corner region. For the composite with the orthotropic substrate, the normal stress shows a very different behavior. It is compressive at the beginning of the corner and tensile at the end of the corner. The maximum magnitude of $\sigma_{n}$ is smaller for the composite with the orthotropic substrate than for the composite with the stiff isotropic substrate. Finally, notice that although the maximum magnitude of the shear stress for the composite with the orthotropic substrate is smaller than for the composite with the stiff isotropic substrate, the shear stress is influenced at a larger distance from the corner for the case of the orthotropic substrate.

From the above discussion it can be concluded that:

- the orthotropy of the substrate has a significant influence on the stresses in substrate and coatings, and 
Table 5.3: Beginning and ending s-coordinate for the corner region for different corner radii.

\begin{tabular}{|c|c|c|}
\hline Radius $[\mathrm{mm}]$ & $s_{\text {begin }}[\mathrm{mm}]$ & $s_{\text {end }}[\mathrm{mm}]$ \\
\hline 0.1 & -0.07854 & 0.07854 \\
0.25 & -0.19635 & 0.19635 \\
0.5 & -0.39270 & 0.39270 \\
0.75 & -0.58905 & 0.58905 \\
1.0 & -0.78540 & 0.78540 \\
\hline
\end{tabular}

- that, most importantly, the region of influence of the corner on the stresses away from the corner is extended for composites with orthotropic substrates.

\subsection{Influence of the Corner Radius}

Finally, the influence of the corner radius on the stress concentration in the corner region and on the far-field stresses was studied. As before, $\mathrm{C} / \mathrm{C}$ substrates with $\mathrm{B}_{4} \mathrm{C}$ and $\mathrm{Si}_{3} \mathrm{~N}_{4}$ coatings applied at the same temperature were considered. The substrate and the coatings were subjected to a $100^{\circ} \mathrm{C}$ temperature increase. The substrate dimensions were $w_{s u b}=4 \mathrm{~mm}$ and $t_{s u b}=1 \mathrm{~mm}$. The corner radius was varied from $r=0.1 \mathrm{~mm}$ to $r=1 \mathrm{~mm}$. Both coating thicknesses were $t_{\text {coat }}=0.05 \mathrm{~mm}$.

In the presentation of the results the $s$-coordinate was offset such that the center of the corner would map to $s=0$ for all cases. The $s$ locations for the beginning and the end of the corner for the different cases are given in Table 5.3. The stress components $\sigma_{s}, \sigma_{n}$, $\sigma_{z}$, and $\tau_{s n}$ at the center of the $\mathrm{Si}_{3} \mathrm{~N}_{4}$ and $\mathrm{B}_{4} \mathrm{C}$ coatings are shown in Figs. 5.19 and 5.20, respectively. Details of the normal and shear stresses for both coatings in the corner region are shown in Figs. 5.21 - 5.24. Because the overall behavior of the stresses in both coatings is similar, only the stresses in the $\mathrm{Si}_{3} \mathrm{~N}_{4}$ coating are discussed in detail.

First consider the tangential stress $\sigma_{s}$ in Fig. 5.19. For all corner radii considered, this stress approaches the same value away from the corner. However, in the corner region the stress strongly depends on the corner radius. For a larger corner radius, as the corner is 


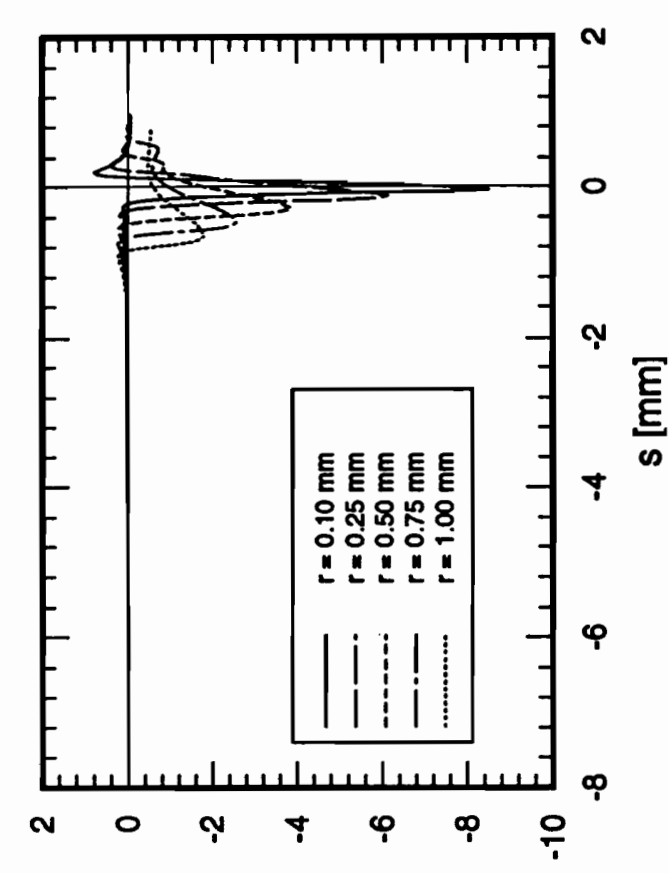

\section{[edw] ${ }^{\circ}$}

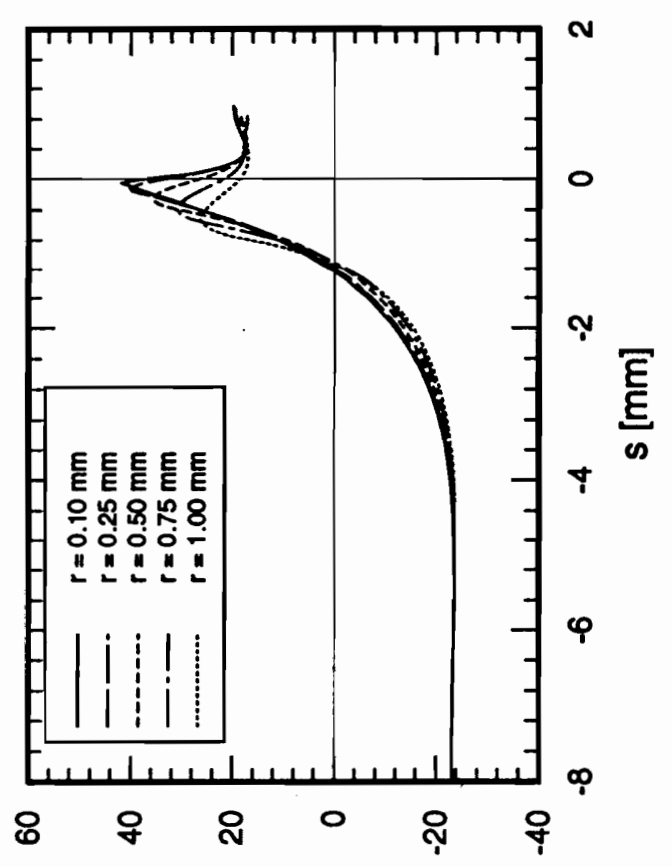

$$
\left[e_{d W}\right]^{s}
$$$$
\left[e_{d W}\right]_{2}
$$

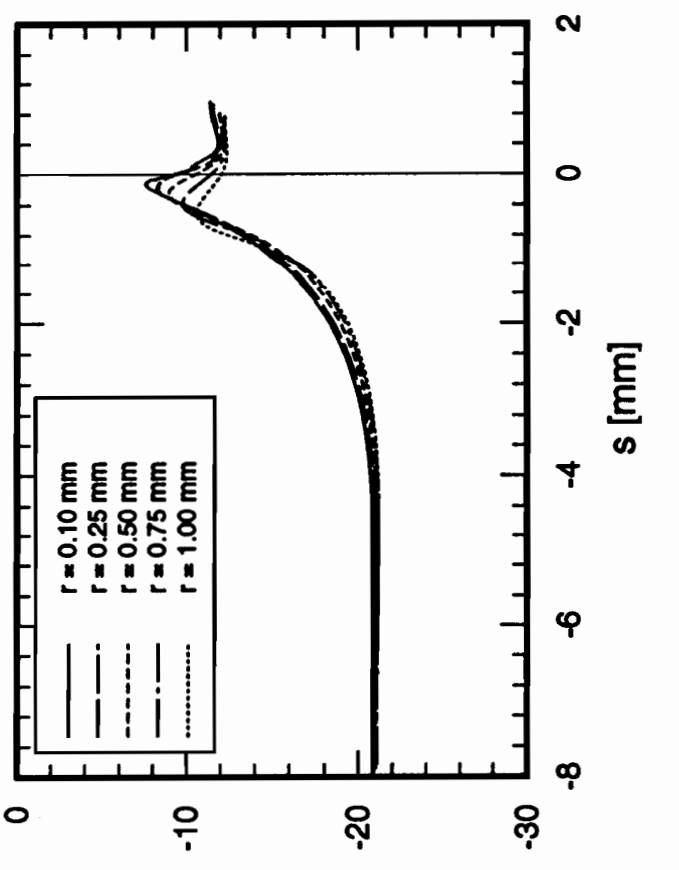

$\left[e_{d W}\right]^{z}$ 


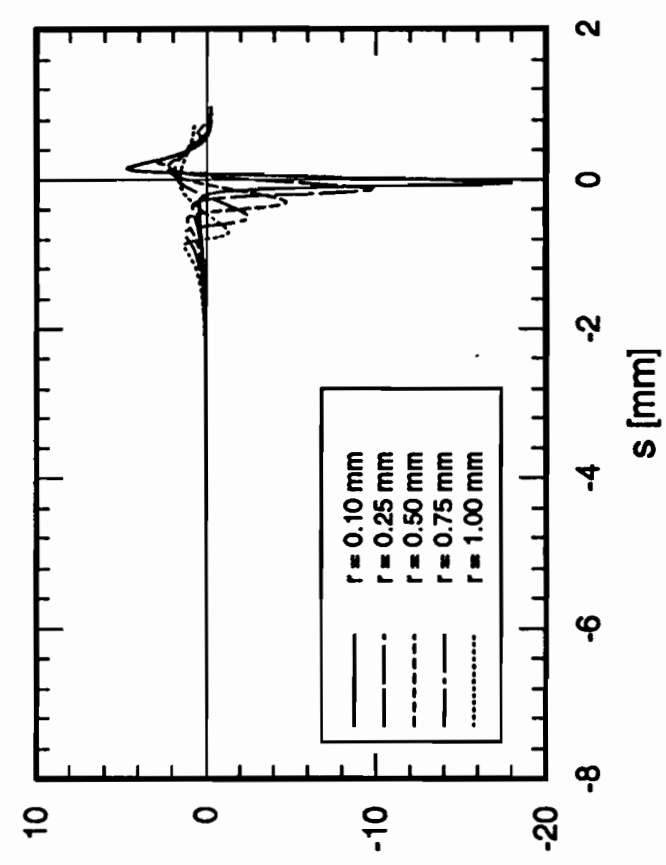

[edW] ${ }^{\mathrm{o}}$

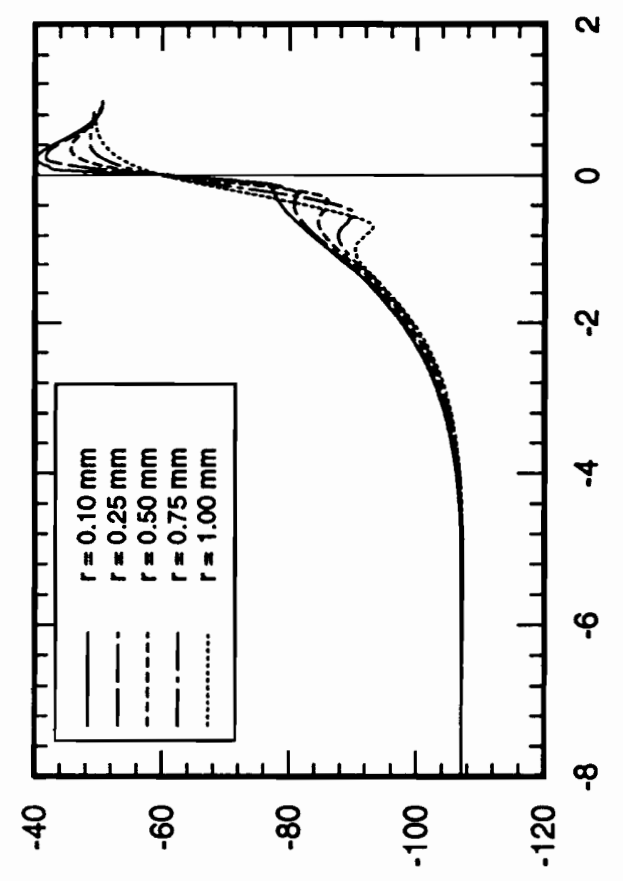

$\left[e_{d W}\right]^{s}$

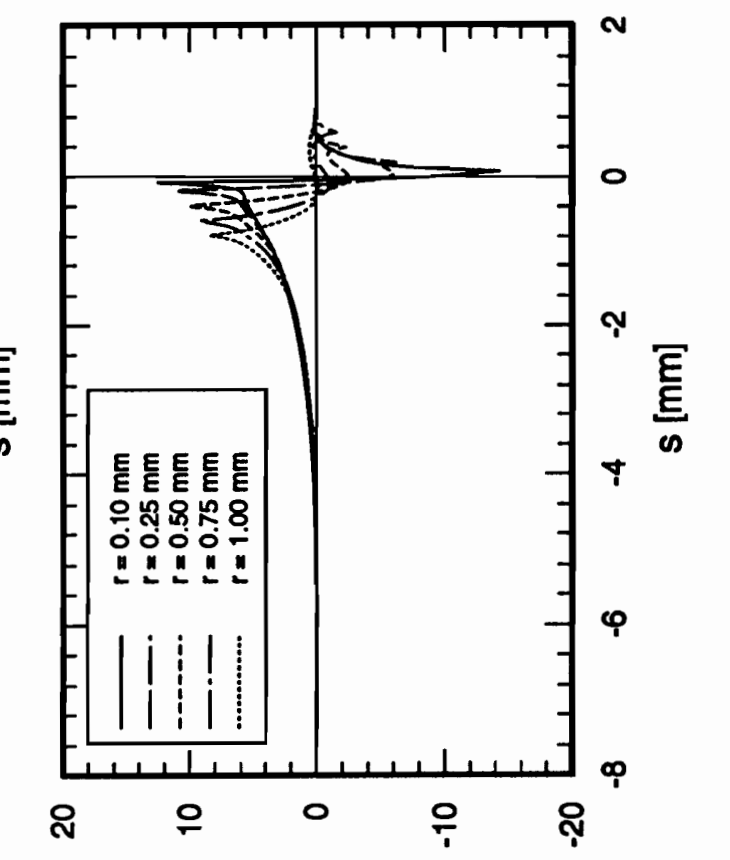

$\left[e_{d W}\right]_{2}^{\text {ss }}$

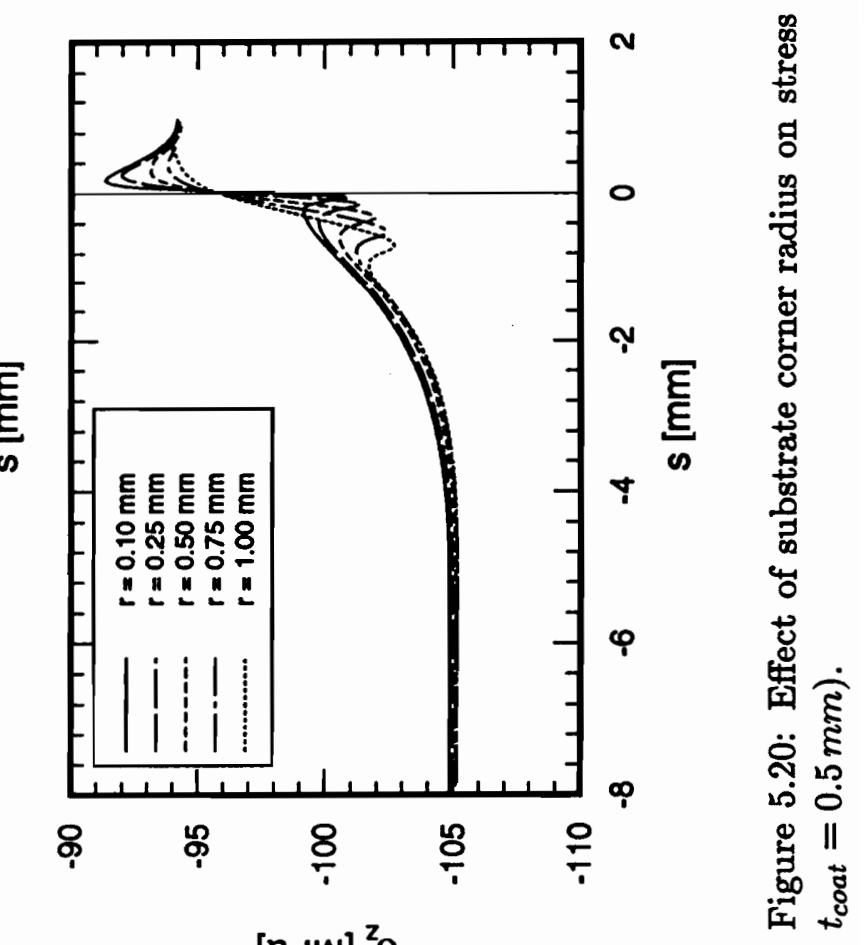

$\left[e_{d W}\right]^{z}$ 


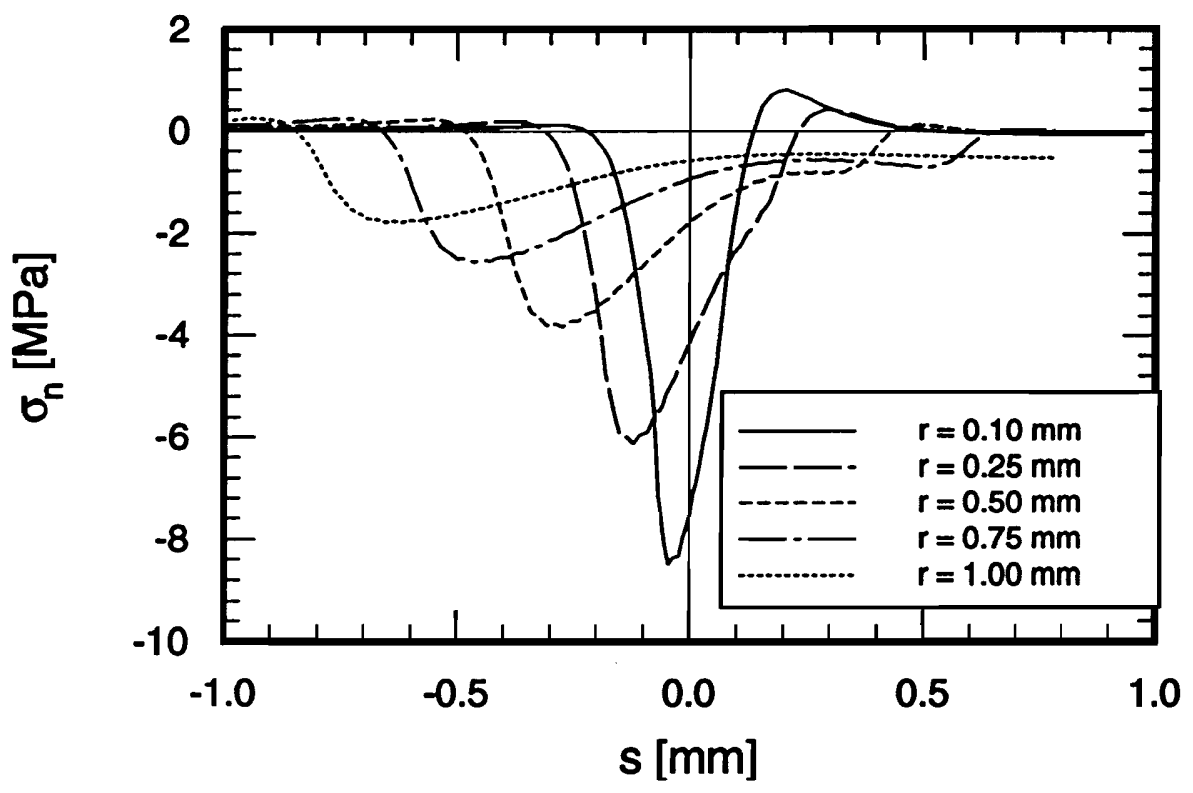

Figure 5.21: Details of normal stress $\sigma_{n}$ in $\mathrm{Si}_{3} \mathrm{~N}_{4}$ coating for different substrate corner radii $\left(w_{s u b}=8 \mathrm{~mm}, t_{s u b}=1 \mathrm{~mm}, t_{\text {coat }}=0.5 \mathrm{~mm}\right)$.

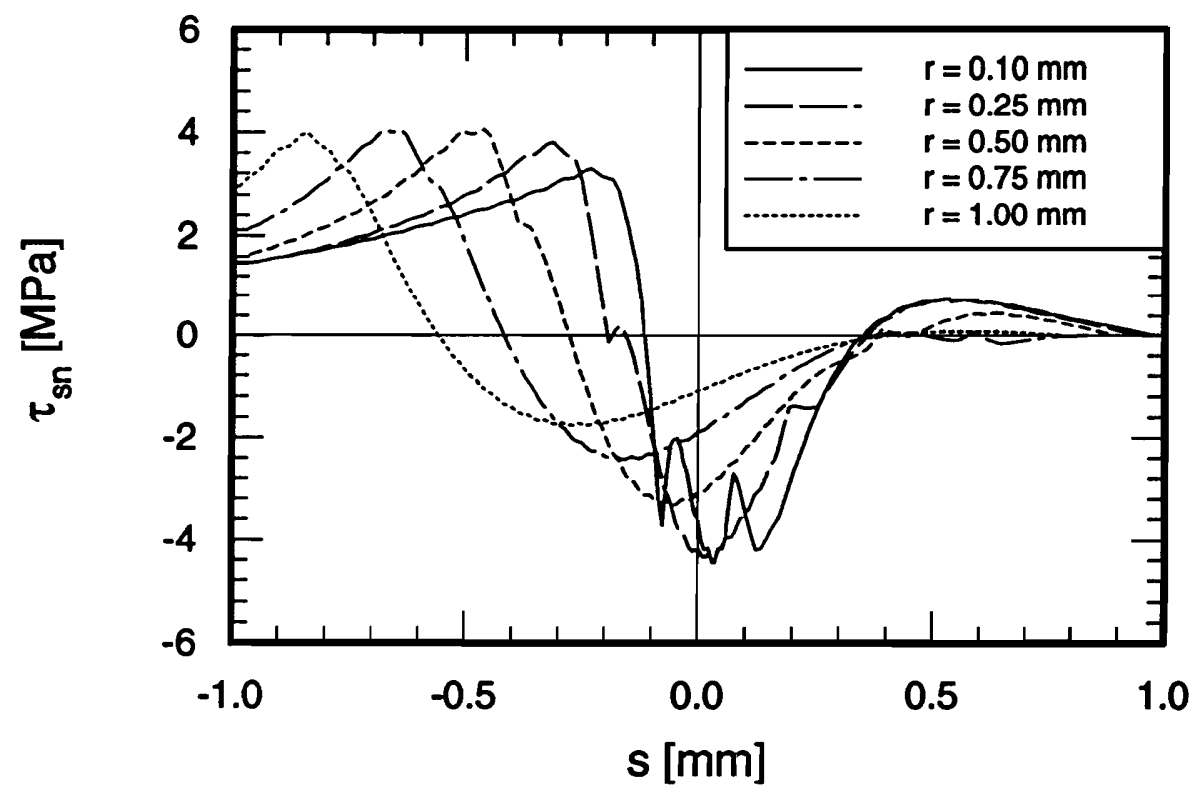

Figure 5.22: Details of shear stress $\tau_{s n}$ in $\mathrm{Si}_{3} \mathrm{~N}_{4}$ coating for different substrate corner radii $\left(w_{s u b}=8 \mathrm{~mm}, t_{\text {sub }}=1 \mathrm{~mm}, t_{\text {coat }}=0.5 \mathrm{~mm}\right)$. 


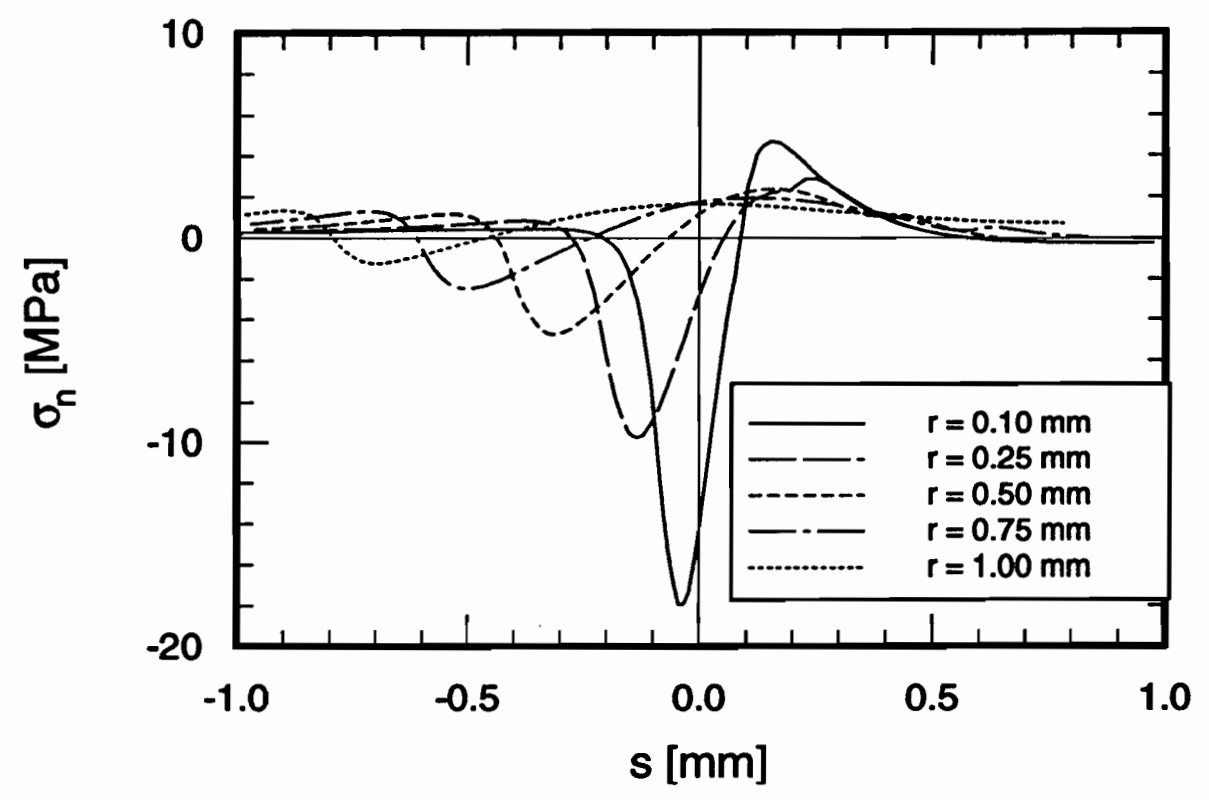

Figure 5.23: Details of normal stress $\sigma_{n}$ in $\mathrm{B}_{4} \mathrm{C}$ coating for different substrate corner radii $\left(w_{\text {sub }}=8 \mathrm{~mm}, t_{\text {sub }}=1 \mathrm{~mm}, t_{\text {coat }}=0.5 \mathrm{~mm}\right)$.

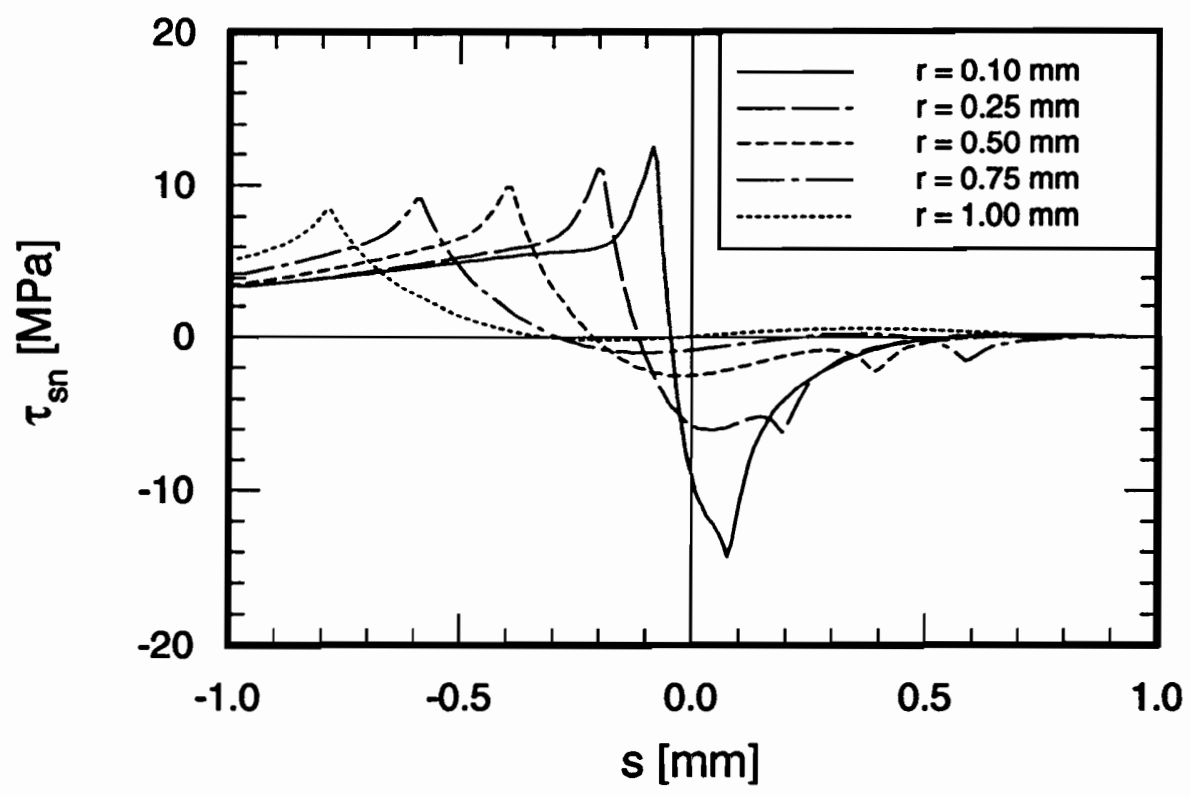

Figure 5.24: Details of shear stress $\tau_{s n}$ in $\mathrm{B}_{4} \mathrm{C}$ coating for different substrate corner radii $\left(w_{s u b}=8 \mathrm{~mm}, t_{s u b}=1 \mathrm{~mm}, t_{\text {coat }}=0.5 \mathrm{~mm}\right)$. 
traversed, the stress makes a smooth transition from its value before the corner to its value after the corner. In contrast, for smaller corner radii large stress concentrations occur in the corner region, almost at the center of the corner $(s=0)$. As the corner is traversed, the stress significantly overshoots the value it reaches after the corner. The same observations hold for the axial stress $\sigma_{z}$. However, for the axial stress the stress concentration for small corner radii is not as severe as for the tangential stress.

As can be seen in Fig. 5.19, the normal stress $\sigma_{n}$ is confined to the corner region for all corner radii. Large stress concentrations are present in the corner region for small corner radii. In Fig. 5.21 the normal stress in the corner region is shown in more detail. For large corner radii the normal stress is almost zero throughout the corner. The stress increases significantly for decreasing corner radii. The increase in the maximum normal stress in the corner region is of higher order than the decrease in corner radius.

As with the normal stress, the shear stress is confined to the corner region. Away from the corner, the stress approaches zero. The more detailed view of the shear stress in the corner region in Fig. 5.22 reveals several interesting aspects of the shear stress distribution. Two stress concentrations are present in the shear stress when traversing the corner from $s$-values "before the corner" to $s$-values "after the corner" (from left to right in Fig. 5.22). One stress concentration occurs directly at the beginning of the corner for the different corner radii. The second stress concentration occurs closer to the center of the corner. The largest stress concentration occurs closer to the center of the corner. The magnitude of the stress concentrations at the beginning of the corner are almost independent of the corner radius. In contrast, the stress concentration near the center of the corner strongly depends on the corner radius. This stress concentration increases for smaller corner radii, and is felt to be due to a coupling of geometric and orthotropic effects. It should be noted that for the $\mathrm{B}_{4} \mathrm{C}$ coatings, the largest stress concentrations in the shear stress occurs before the corner rather than close to the center, as was the case for the $\mathrm{Si}_{3} \mathrm{~N}_{4}$ coating. However, unlike the situation for the $\mathrm{Si}_{3} \mathrm{~N}_{4}$ coating, the shear stress concentration at the beginning of the corner increases with decreasing corner radius, as does the stress concentration near the center of 
the corner.

From this discussion the following conclusions can be drawn:

- Stresses away from the corner are not affected by the corner radius.

- The shear and normal stress are confined to the corner region. Both stresses diminish rapidly when moving away from the corner.

- Large stress concentrations are present in the corner region for all stress components. The stress concentrations increase for decreasing radius.

- The increase in the stress concentration for the normal stress is of higher order than the decrease in corner radius.

The large stress concentrations in the corner region for small corner radii may cause premature failure of coatings. Therefore, it is clear that as large as possible corner radii should be used in substrates to reduce the stress concentrations.

\subsection{Summary}

In this chapter the features and characteristics of the stress distributions in the coatings and substrate were presented. Also, the influence of some geometric parameters on the stresses in the substrate and coating was studied. Though these studies were for temperatureindependent material properties, qualitatively the characteristics of the influence of the various parameters on the stress distributions should extrapolate to temperature-dependent analyses. In the next chapter results for temperature-dependent material properties are presented and the influence of several material parameters on the stresses is studied. 


\section{Chapter 6}

\section{Temperature-Dependent Results}

While temperature-independent analysies are simpler and provide reasonable insight into the thermal stresses in coatings, realistic materials exhibit temperature-dependent effects. These must be included and in this chapter results from the temperature-dependent analysis are shown. Single-layer, multilayer coatings, and gradient coatings were considered. The influence of various parameters on the stresses was studied. The parameters varied included the coefficients of thermal expansion and the moduli of the coatings, the application temperature of the coatings, as well as different property variations through the thickness of gradient coatings. More detailed descriptions of the parameter studies are given in individual sections. To compare results from the parametric studies, two baseline cases were established. These cases were :

1. A C/C substrate with a $\mathrm{SiC}$ coating applied at $1200^{\circ} \mathrm{C}$.

2. A C/C substrate with a $\mathrm{B}_{4} \mathrm{C}$ coating applied at $1000^{\circ} \mathrm{C}$ and a $\mathrm{Si}_{3} \mathrm{~N}_{4}$ coating applied at $1500^{\circ} \mathrm{C}$.

In both cases, stresses were calculated for a range of temperatures. Results from ISPSAs for infinitely wide composites and from finite element analyses for composites with a corner were obtained for both cases. The results obtained from the ISPSAs were used to normalize results obtained in the parametric studies. 

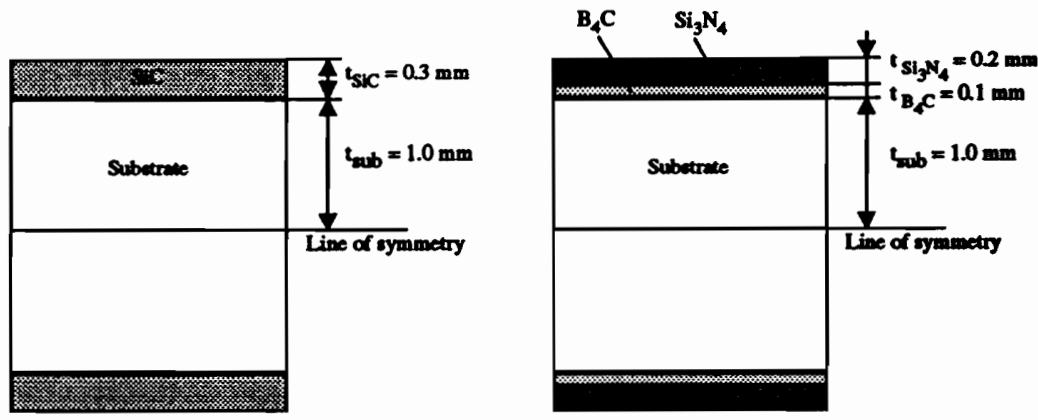

Figure 6.1: Baseline models for ISPSA.

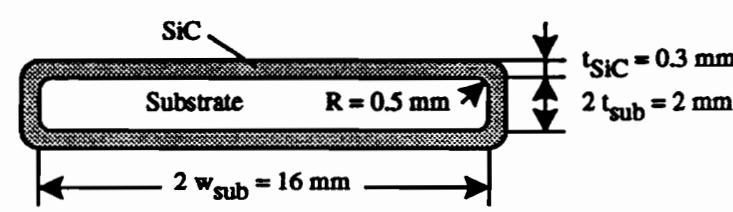

(a) Dimensions of substrate with SiC coating.

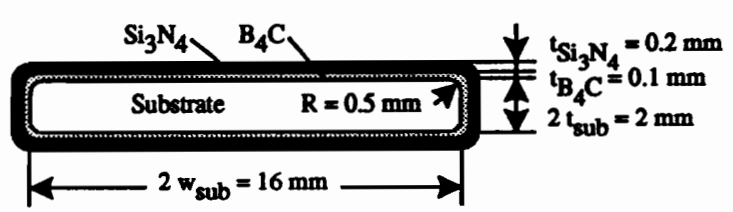

(b) Dimensions of substrate with $\mathrm{B}_{4} \mathrm{C}$ and $\mathrm{SiC}$ coatings.

Figure 6.2: Baseline models for cases including a corner.

\subsection{The Baseline Cases}

The first baseline case was a $\mathrm{C} / \mathrm{C}$ substrate with a $\mathrm{SiC}$ coating applied at $1200^{\circ} \mathrm{C}$. The half-thickness of the substrate was $t_{s u b}=1 \mathrm{~mm}$ and the thickness of the single coating was $t_{S i C}=0.3 \mathrm{~mm}$. The second baseline case was a $\mathrm{C} / \mathrm{C}$ composite with a $\mathrm{B}_{4} \mathrm{C}$ coating applied at $1000^{\circ} \mathrm{C}$ and $\mathrm{a} \mathrm{Si}_{3} \mathrm{~N}_{4}$ coating applied at $1500^{\circ} \mathrm{C}$. The substrate thickness was again $t_{s u b}=1 \mathrm{~mm}$ and the coating thickness for the $\mathrm{B}_{4} \mathrm{C}$ and the $\mathrm{Si}_{3} \mathrm{~N}_{4}$ coatings were $t_{B_{4} C}=0.1 \mathrm{~mm}$ and $t_{S_{3} N_{4}}=0.2 \mathrm{~mm}$, respectively. The geometries of the two baseline cases for the ISPSAs are shown in Fig. 6.1. The geometries of the two baseline cases fot the composites with a corner are shown in Fig. 6.2. 


\subsubsection{Incremental Plane-Stress Analyses of Baseline Cases}

As was mentioned in Section 3.1, the $x-y-z$ coordinate system for the ISPSA was chosen such that the $x$ - and the $z$-coordinates are the inplane coordinates and the $y$-coordinate is the transverse coordinate. As can be seen in Fig. 5.1, the $x-y-z$ coordinate system is aligned with the $s-n-z$ coordinate system for values of $s$ before the corner. Because isotropic coatings and cross-ply orthotropic substrates were chosen, the magnitude of the two inplane stress components, $\sigma_{x}$ and $\sigma_{z}$, were the same for the ISPSA. Stresses calculated for a range of temperatures for the two baseline cases after application of all coatings are shown in Fig. 6.3. Numerical values of the stresses for some temperatures are shown in Table 6.1. Notice that the stress in the $\mathrm{B}_{4} \mathrm{C}$ coating for the second baseline case is not zero at its application temperature $\left(1000^{\circ} \mathrm{C}\right)$. This is due to the interaction between the $\mathrm{Si}_{3} \mathrm{~N}_{4}$ coating and the $\mathrm{B}_{4} \mathrm{C}$ coating when the composite is cooled from $1500^{\circ} \mathrm{C}$ after the $\mathrm{Si}_{3} \mathrm{~N}_{4}$ coating is applied. Note also that if temperature-independent material properties were used, the stress-temperature relations would all be linear. Because cracking of coatings is not modeled in the present study, some of the stresses listed in Table 6.1 are higher than the strength of the respective materials. This is in agreement with experimental analyses reported in literature and discussed in Chapter 1, which show that single material coatings actually may crack at low temperatures. Even though some of the calculated stresses exceed the material strength, results shown in the following studies are useful in revealing the nature of the stresses in substrate and coatings, and the influence of different parameters on the stresses. Therefore these results contribute significantly to the understanding of the behavior of coated $\mathrm{C} / \mathrm{C}$ substrates and the results can be used for the design of analyses with more complex material models, as well as in the study of concepts for stress reduction in coatings. These stresses calculated using the ISPSA are baseline, or reference, stresses that will be used throughout this chapter as a basis for comparison. Unless otherwise noted, all stresses at a particular temperature discussed in the following studies are normalized by the stresses given in Table 6.1. The normalized stresses are denoted by an overbar, e.g., $\bar{\sigma}_{s}$ 


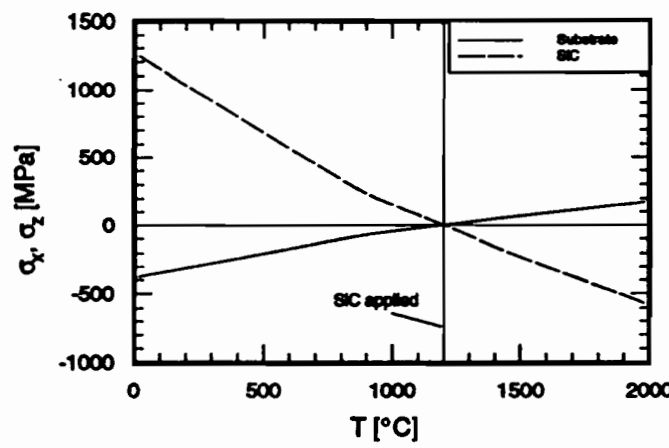

(a) Stresses in $\mathrm{C} / \mathrm{C}$ substrate with $\mathrm{SiC}$ coating.

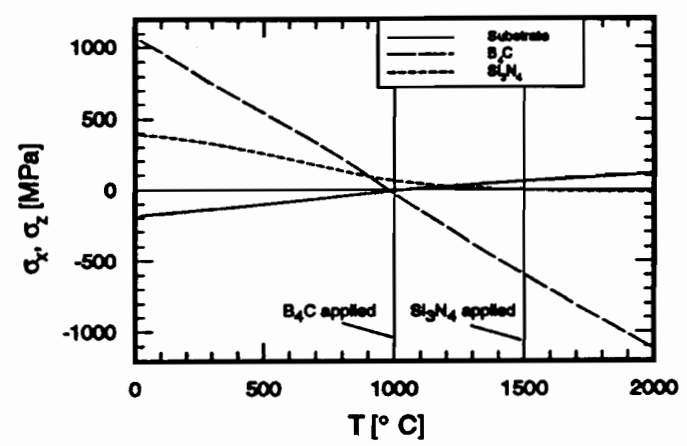

(b) Stresses in $\mathrm{C} / \mathrm{C}$ substrate with $\mathrm{B}_{4} \mathrm{C}$ and $\mathrm{SiC}$ coatings.

Figure 6.3: Temperature-dependent stresses for baseline cases.

Table 6.1: Temperature-dependent stresses from ISPSA for baseline cases.

\begin{tabular}{|c|c|c|r|r|r|r|r|}
\hline \multicolumn{3}{|c|}{ Temperature $\left[{ }^{\circ} \mathrm{C}\right]$} & 20 & 500 & 1000 & 1500 & 2000 \\
\hline \multirow{4}{*}{$\sigma_{x}, \sigma_{z}$} & \multirow{2}{*}{ Case 1 } & $\mathrm{SiC}$ & 1241.4 & 681.37 & 153.48 & -229.01 & -580.18 \\
& & $\mathrm{Sub}$. & -372.42 & -204.41 & -46.044 & 68.702 & 174.06 \\
\cline { 2 - 8 }$[\mathrm{MPa}]$ & \multirow{3}{*}{ Case 2 } & $\mathrm{Si}_{3} \mathrm{~N}_{4}$ & 391.17 & 253.303 & 62.6882 & 0. & -13.15 \\
& & $\mathrm{~B}_{4} \mathrm{C}$ & 1050.27 & 540.135 & -28.379 & -596.87 & -1121.5 \\
& & $\mathrm{Sub}$. & -183.26 & -104.67 & -9.6997 & 59.687 & 114.78 \\
\hline
\end{tabular}

is the normalized stress $\sigma_{s}$.

\subsection{Comparison of Temperature-Dependent and Tempera- ture-Independent Analyses of Baseline Cases}

To demonstrate the importance of including temperature-dependent properties in the analysis, results from temperature-dependent and temperature-independent ISPSAs for both baseline cases were compared.

The stresses in the $\mathrm{SiC}$ coating and the $\mathrm{C} / \mathrm{C}$ substrate for the first baseline case are 
shown in Fig. 6.4. The stress- and strain-free temperature of the substrate and the coating is indicated in the figure by the vertical line at $1200^{\circ} \mathrm{C}$. It can be seen that the temperatureindependent analysis significantly underpredicts stresses at low temperatures and overpredicts stresses at high temperatures.

For the second baseline case stresses in the coatings and the substrate are shown in Fig. 6.5, in which the stress- and strain-free temperatures are indicated. As was mentioned earlier, the stresses shown are the stresses calculated after the application of the $\mathrm{Si}_{3} \mathrm{~N}_{4}$ coating. For the substrate the temperature-independent analysis underpredicts stresses at room temperature. At high temperatures, the temperature-independent results are close to the temperature-dependent results. In the $\mathrm{B}_{4} \mathrm{C}$ coating the difference in stresses calculated using both theories is very small over the entire temperature range. In contrast, in the $\mathrm{Si}_{3} \mathrm{~N}_{4}$ coating the difference in stresses is very large. At room temperature the temperatureindependent analysis underpredicts stresses by $25 \%$ compared to the temperature-dependent analysis.

From this study it can be concluded that:

- To obtain accurate stresses a temperature-dependent analysis has to be used.

- The temperature-independent analysis may significantly underpredict stresses and hence lead to wrong conclusions about coating failure.

- To justify a temperature-independent analysis all stresses need to be checked. In the present case just checking the stresses in the $\mathrm{B}_{4} \mathrm{C}$ coating could lead to the conclusion that the simpler temperature-independent analysis would be sufficient, while the stresses in the $\mathrm{Si}_{3} \mathrm{~N}_{4}$ coating clearly show that a temperature-dependent analysis has to be used. 


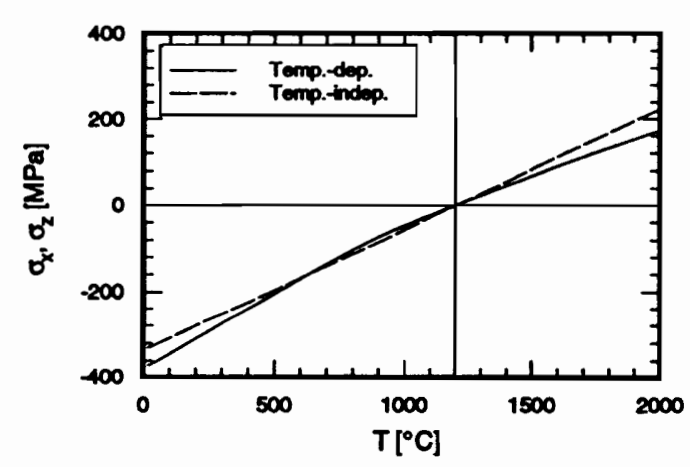

(a) Substrate.

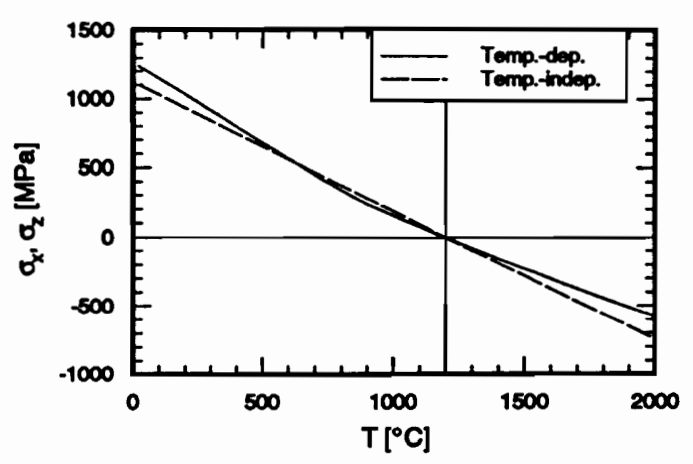

(b) SiC coating.

Figure 6.4: Comparison of temperature-independent and temperature-dependent stresses at different temperatures for a substrate with a $\mathrm{SiC}$ coating.

\subsection{Temperature-Dependent Stresses in a Carbon-Carbon Substrate with a $\mathrm{SiC}$ coating}

The stresses in the coating on a $\mathrm{C} / \mathrm{C}$ substrate with a $\mathrm{SiC}$ coating and a corner were analyzed in detail. The geometry is shown in Fig. 6.2a and the material properties are given in Appendix A. Stresses were calculated at $20^{\circ} \mathrm{C}, 500^{\circ} \mathrm{C}, 1000^{\circ} \mathrm{C}$, and $1500^{\circ} \mathrm{C}$ after both coatings were applied.

First, consider the stresses at room temperature $\left(20^{\circ} \mathrm{C}\right)$. The normalized tangential, axial, normal, and shear stress components, $\bar{\sigma}_{s}, \bar{\sigma}_{z}, \bar{\sigma}_{n}$, and $\bar{\tau}_{s n}$ in the SiC coating are shown in Fig. 6.6 on page 99. Each stress component for the $\mathrm{SiC}$ was normalized by the stress $\sigma_{x}$ calculated at $T=20^{\circ} \mathrm{C}$ for the $\mathrm{SiC}$ in an infinitely wide composite using the ISPSA. Similarly, each stress component for the substrate was normalized by the stress $\sigma_{x}$ calculated at $T=20^{\circ} \mathrm{C}$ for the substrate in an infinitely wide composite using the ISPSA. These stresses are given in Table 6.1. The stress components are shown as functions of the tangential coordinate $s$ at different locations through the thickness of the $\mathrm{SiC}$ coating. The 


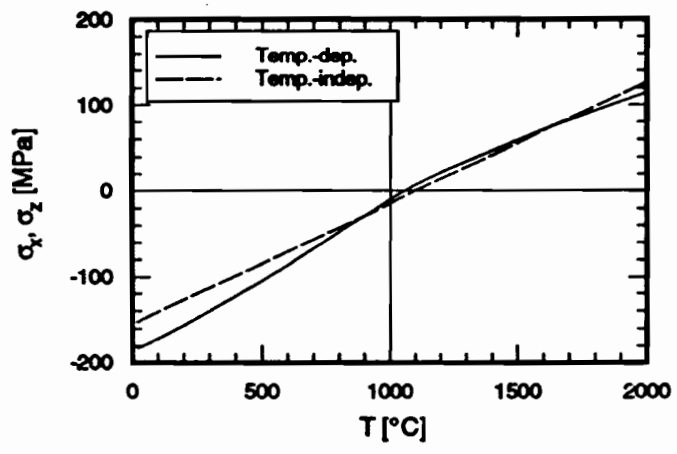

(a) Substrate.

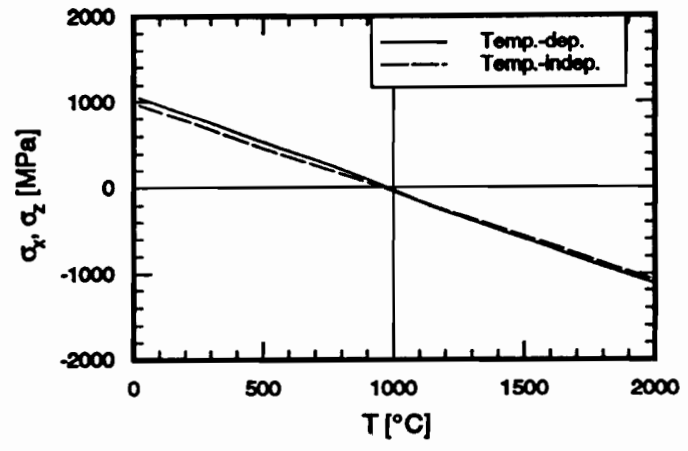

(b) $\mathrm{B}_{4} \mathrm{C}$ coating.

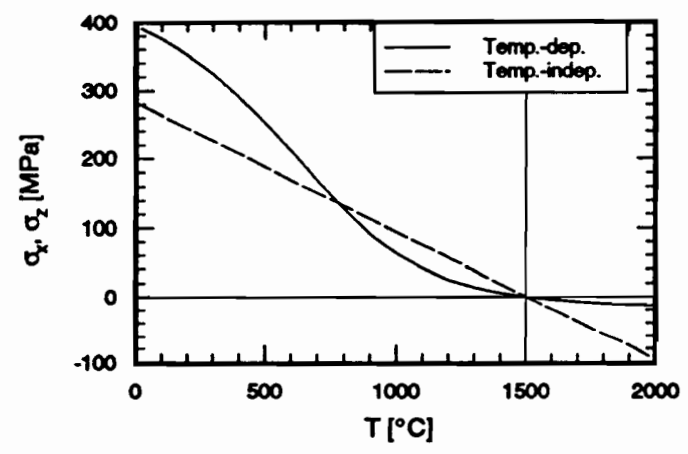

(c) $\mathrm{Si}_{3} \mathrm{~N}_{4}$ coating.

Figure 6.5: Comparison of temperature-independent and temperature-dependent stresses at different temperatures for a substrate with a $\mathrm{Si}_{3} \mathrm{~N}_{4}$ coating and a $\mathrm{B}_{4} \mathrm{C}$ coating. 
distance $d$ in the legend indicates the normalized distance into the coating. The distance $d$ is normalized by the total coating thickness, $d=0$ at the free surface and $d=1$ at the SiC-substrate interface. Through-the-thickness gradients in the stress components are proportional to the distance between the lines for the different thickness locations in the figures. Thus, if the lines for all thickness locations coincide, the particular stress component is uniform through the thickness of a coating. Gradients in the tangential direction ( $s$ direction) are proportional to the slope of the curves in the graphs.

Consider the normalized tangential stress $\bar{\sigma}_{s}$ in the $\mathrm{SiC}$ coating shown in Fig. 6.6. Away from the corner $\bar{\sigma}_{s}=1$ for all thickness locations. This shows that away from the corner the tangential stress approaches the far field value and is uniform through the thickness of the coating. In contrast, in the corner region, the normalized tangential stress varies between $\bar{\sigma}_{s}=-0.32$ near the free surface and $\bar{\sigma}_{s}=1.5$ near the SiC-substrate interface. Thus, a very large gradient through the thickness of the coating is present in the corner region. Also, there is a rapid variation with $s$. The maximum stress in the corner region is approximately $50 \%$ larger than the far field stress. This indicates that the corner causes a strong stress concentration that may lead to premature failure of the coating in the corner region. Further notice that the variation of the stress through the thickness of the coating near the horizontal line of symmetry of the composite is opposite to this variation in the corner region. In other words, near the horizontal line of symmetry the stress is tensile near the free surface $(d=0.02)$ and compressive near the SiC-substrate interface $(d=0.98)$, whereas at the beginning of the corner the stress is compressive near the free surface aind tensile near the interface. The tensile stress in the corner is higher than the tensile stress near the horizontal line of symmetry. Therefore failure should initiate in the corner region near the SiC-substrate interface.

The axial stress, $\bar{\sigma}_{z}$, shown in Fig. 6.6 varies in a way similar to the tangential stress $\bar{\sigma}_{s}$. The overall change of the axial stress in the corner region is much lower than it is for the tangential stress. The stress is tensile for all thickness locations in the corner region. The lower overall change in $\bar{\sigma}_{z}$ is due to the fact that the variation of the axial stress within the 
coating is caused by Poisson effects only.

As expected, the normal stress $\bar{\sigma}_{n}$ in Fig. 6.6 is confined to the corner region. It is zero near the free surface and increases towards the SiC-substrate interface. For thickness locations close to the free surface, the normal stress changes from a tensile stress to a compressive stress traversing the corner with an increasing s-coordinate. For thickness locations close to the $\mathrm{SiC}$-substrate interface, the stress is compressive throughout the corner region. Because of the compressive nature of the normal stress near the SiC-substrate interface, delamination of substrate and coating is not expected.

Like the normal stress, the shear stress, $\bar{\tau}_{s n}$, in Fig. 6.6 is zero away from the corner. In the corner region, stress concentrations in the shear stress component are present near the beginning of the corner and near the center of the corner. For all $s$ locations within the corner, the shear stress is zero near the free surface, increases towards the center of the coating, and then decreases again towards the SiC-substrate interface. The maximum value of the shear stress reaches about $16 \%$ of the far field tangential stress value. The shear stress decreases much more slowly than the normal stress when moving away from the corner toward $s=0$.

In Fig. 6.7 the normalized maximum principal stress, $\bar{\sigma}_{\max }$, and the normalized maximum shear stress, $\bar{\tau}_{\max }$, are shown for the $\mathrm{SiC}$ coating. The distribution of $\bar{\sigma}_{\max }$ is almost identical to the distribution of the normalized tangential stress $\bar{\sigma}_{s}$ shown in Fig. 6.6. Thus, the above discussion for the tangential stress equally applies to the maximum principal stress. The distribution of the maximum shear stress is qualitativeiy similar to the distribution of the maximum principal stress. In the far field the maximum shear stress is, in fact, exactly one half the maximum principal stress. This has to be the case, because the maximum shear stress is $\tau_{\max }=0.5\left(\sigma_{I}+\sigma_{I I}\right)$, where $\sigma_{I}$ and $\sigma_{I I}$ are the principal stresses. In the far field $\sigma_{I}=\sigma_{\max }$ and $\sigma_{I I}=0$. Therefore, in the far field $\tau_{\max }=0.5 \sigma_{\max }$. Because the maximum principal stress component is the dominant stress component for all $s$-locations and $\tau_{\max }=0.5\left(\sigma_{I}+\sigma_{I I}\right)$, the distribution of the maximum shear stress is similar to the distribution of the maximum principal stress. Because the distribution of the 
maximum principal stress is similar to the distribution of the tangential stress, from this point on discussions will focus on the more intuitive stresses, $\bar{\sigma}_{s}, \bar{\sigma}_{n}, \bar{\sigma}_{z}$, and $\bar{\tau}_{s n}$ in the $s-n$ - $z$-coordinate system.

In Figs. 6.8 - 6.10 the normalized stress components in the $\mathrm{SiC}$ coating are shown at $500^{\circ} \mathrm{C}, 1000^{\circ} \mathrm{C}$, and $1500^{\circ} \mathrm{C}$. All stress components at $500^{\circ} \mathrm{C}$ were normalized by the stress in the $\mathrm{SiC}$ coating at $500^{\circ} \mathrm{C}$ shown in Table 6.1, all stress components at $1000^{\circ} \mathrm{C}$ were normalized by the stress in the $\mathrm{SiC}$ coating at $1000^{\circ} \mathrm{C}$ shown in Table 6.1, etc. A comparison of Figs. 6.8 - 6.10 with Fig. 6.6 shows that the distributions of the normalized stresses are almost identical for all temperatures. Notice, however, that the positive normalized stresses in the figures for $1500^{\circ} \mathrm{C}$ indicate compressive stresses instead of the tensile stresses which occured for the other temperatures. This is the case because the stresses for $1500^{\circ} \mathrm{C}$ were normalized by a compressive stress (see Table 6.1).

Because the normalized stress distributions are similar at all temperatures, in the following sections only stresses at $20^{\circ} \mathrm{C}$ will be considered. These room temperature stresses are the most significant stresses because at this temperature the tensile tangential stress in the $\mathrm{SiC}$ coating is larger than at higher temperatures. If this stress can be reduced to a level at which the coating will not fail at $20^{\circ} \mathrm{C}$, then the coating also will not fail at higher temperatures. As was shown, the largest stresses occur in the corner region. A large stress concentration was present in the tangential stress $\bar{\sigma}_{s}$ in the $\mathrm{SiC}$ coating near the SiC-substrate interface. To obtain crack-free coatings, it is important to reduce this stress concentration. Therefore in the following sections concepts to reduce these stress concentrations are studied. These concepts include the use of compliant interlayers, or isolation layers, and the use of gradient coatings. 

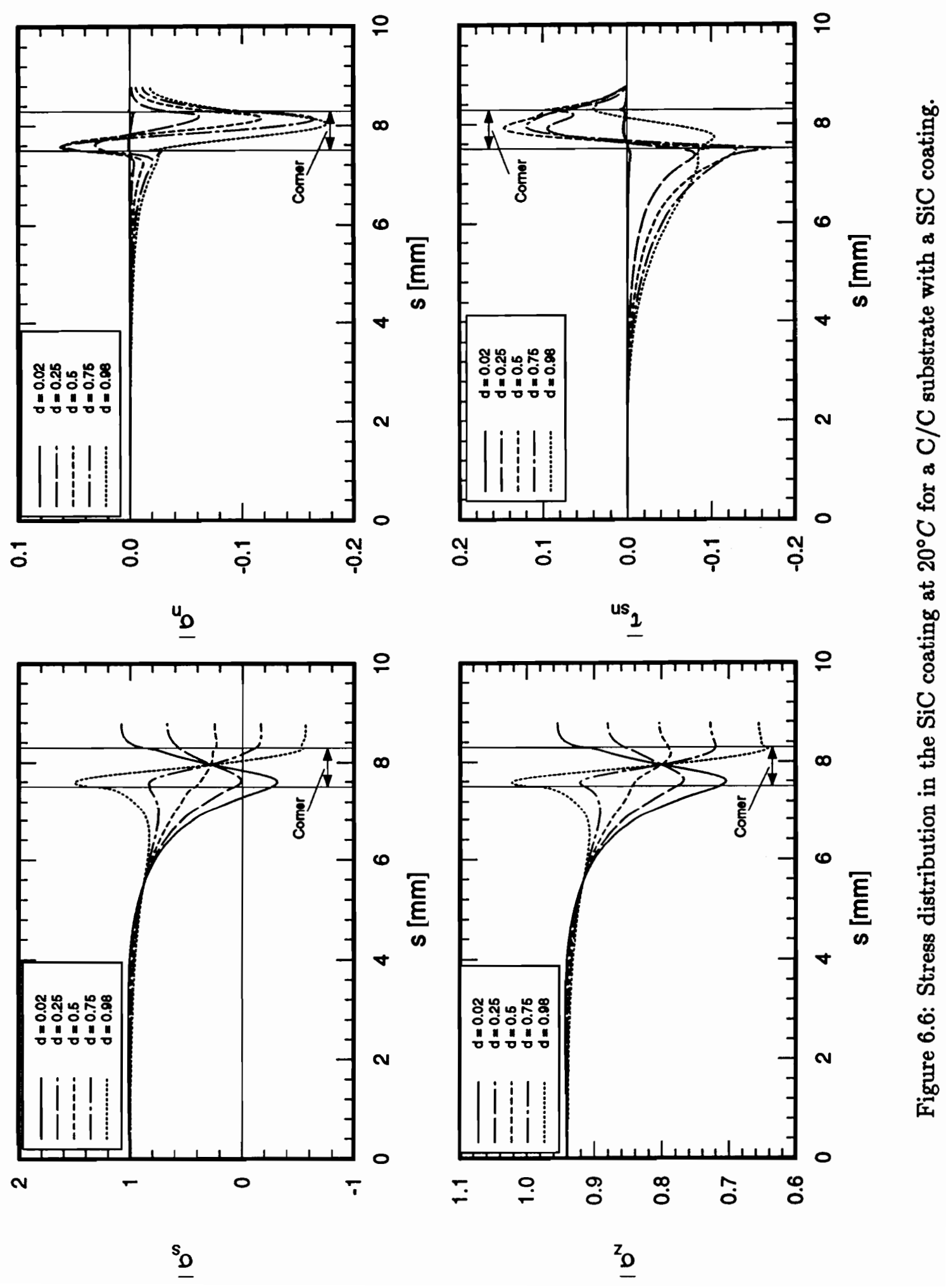

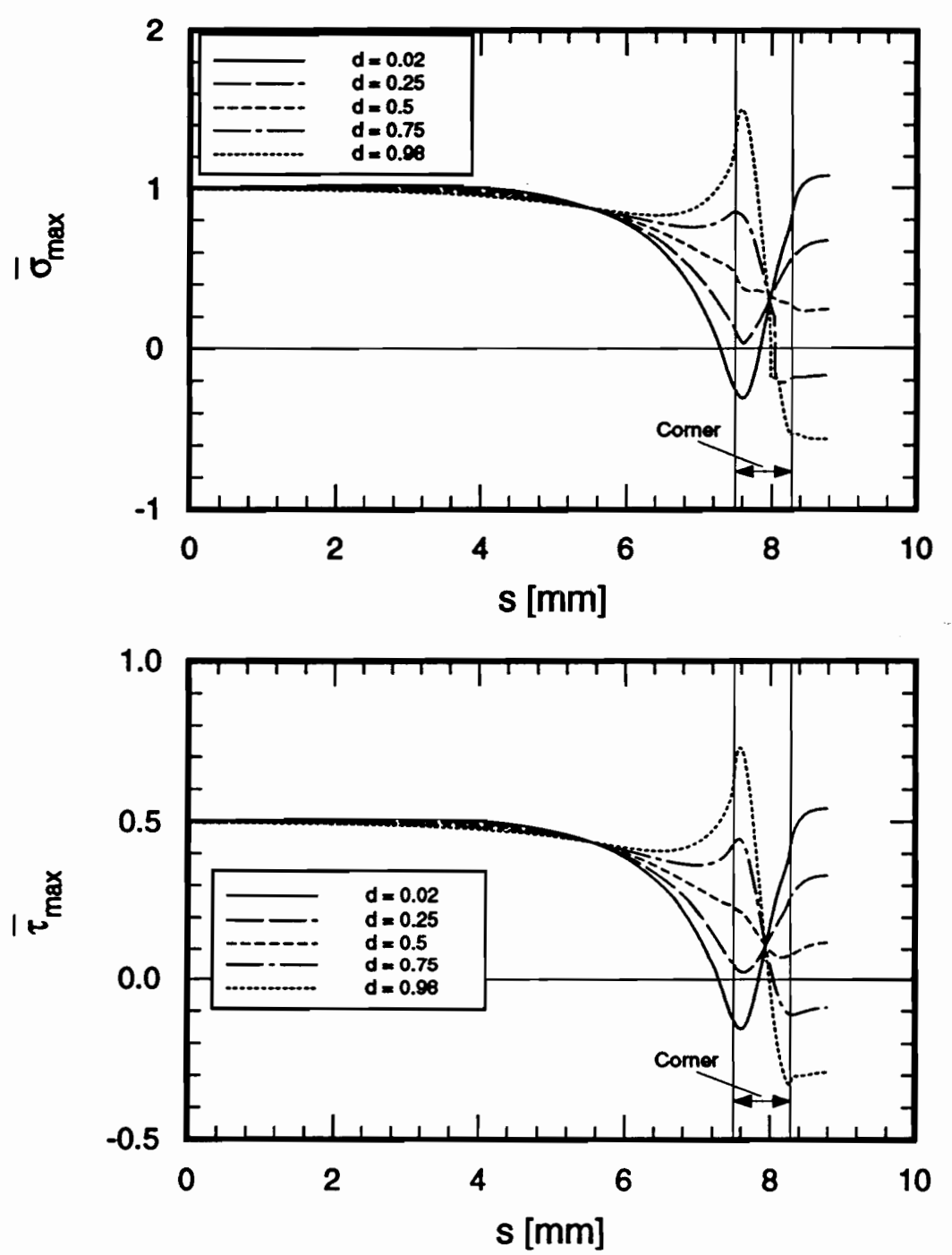

Figure 6.7: Maximum principal stress and maximum shear stress at $20^{\circ} \mathrm{C}$ in the $\mathrm{SiC}$ coating for a $\mathrm{C} / \mathrm{C}$ substrate with a $\mathrm{SiC}$ coating. 

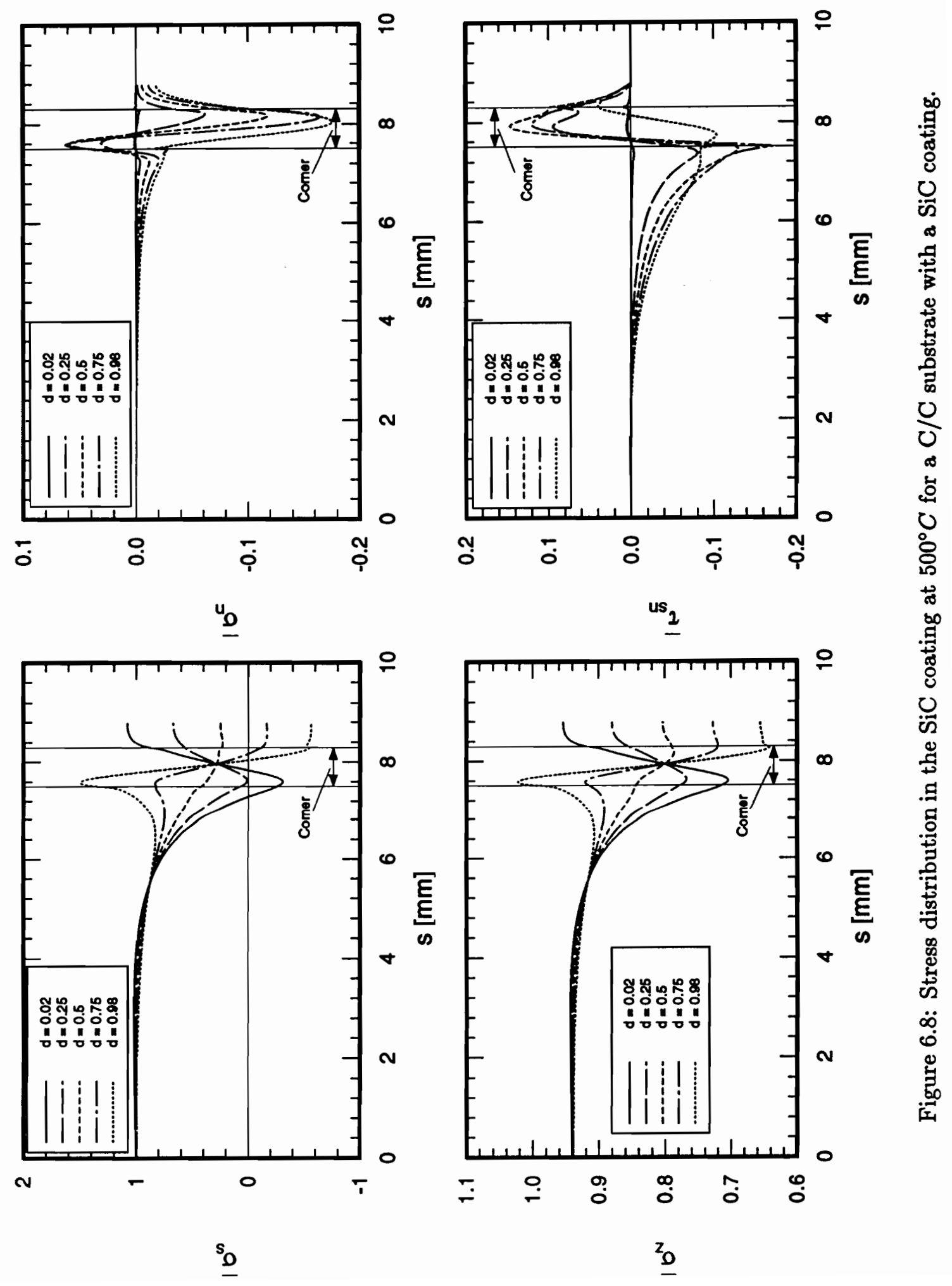

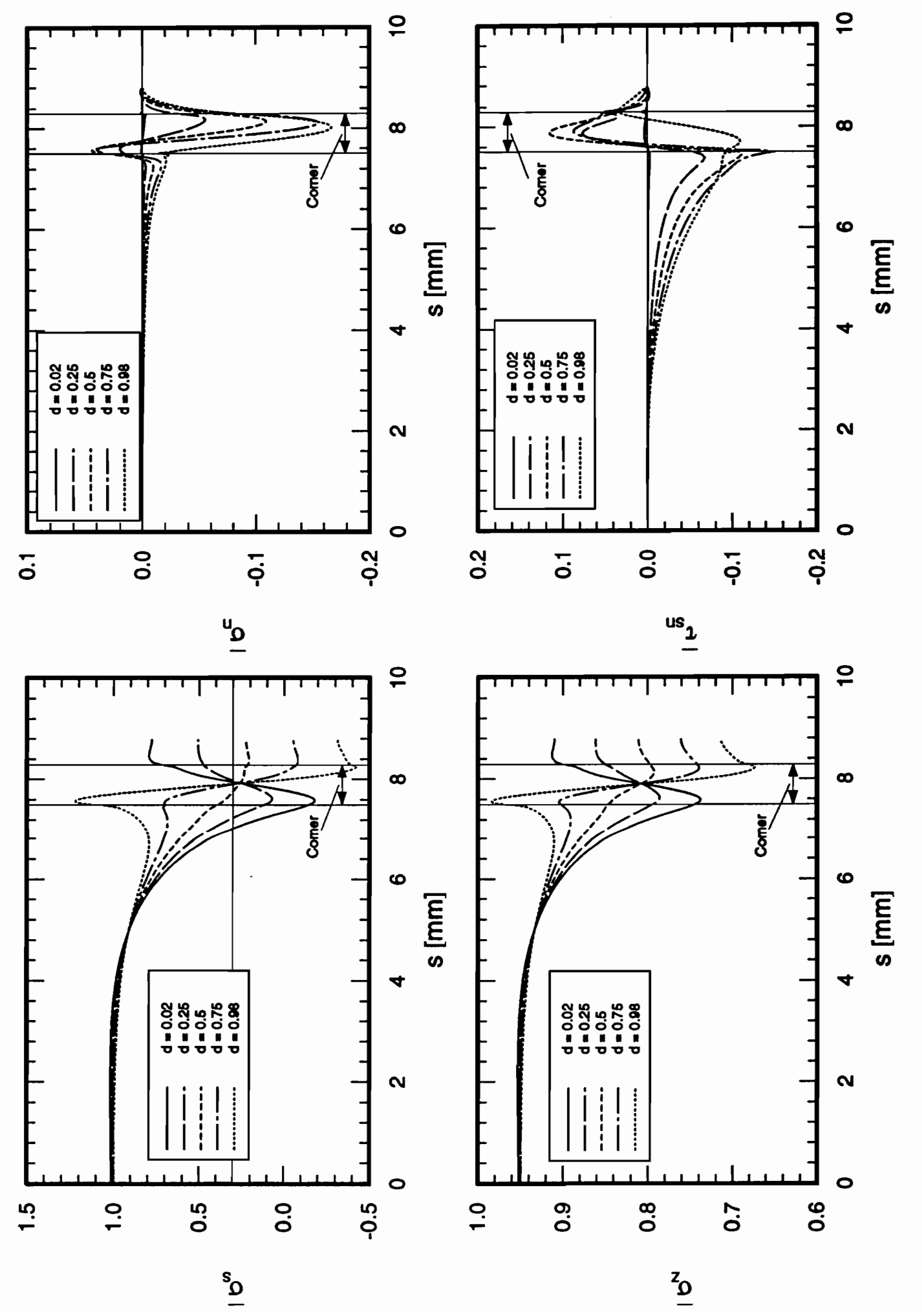



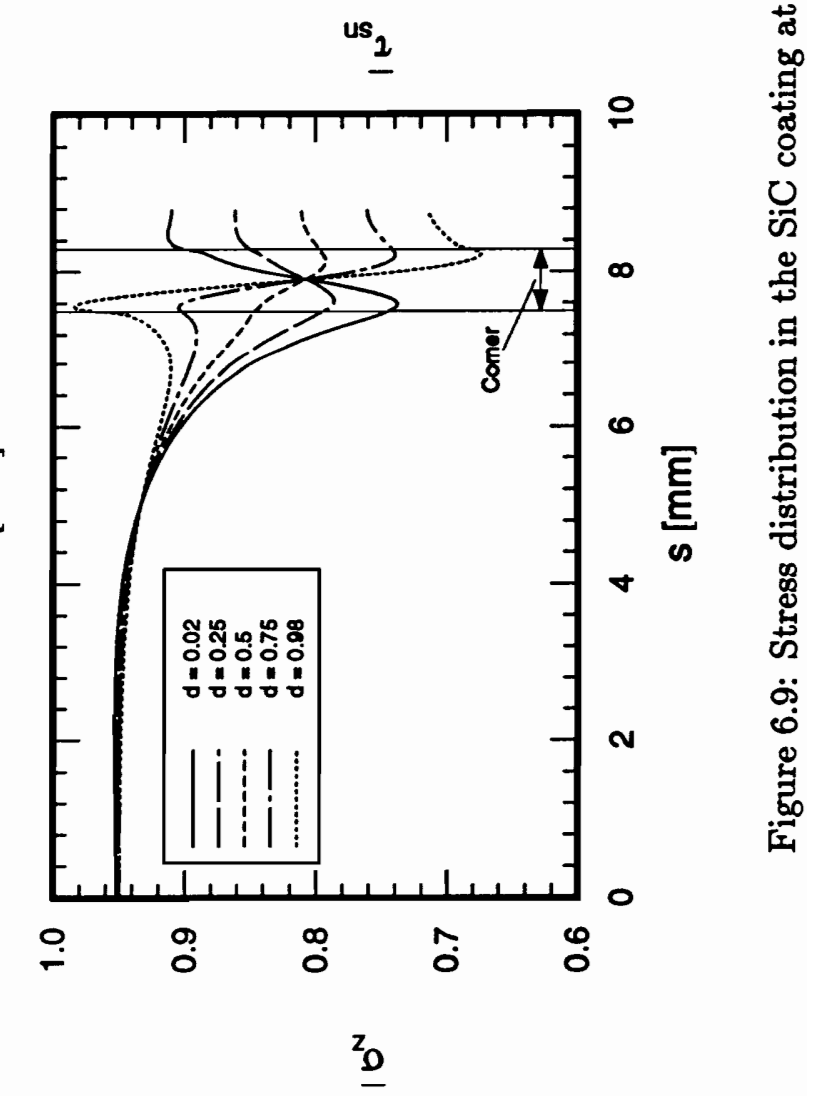



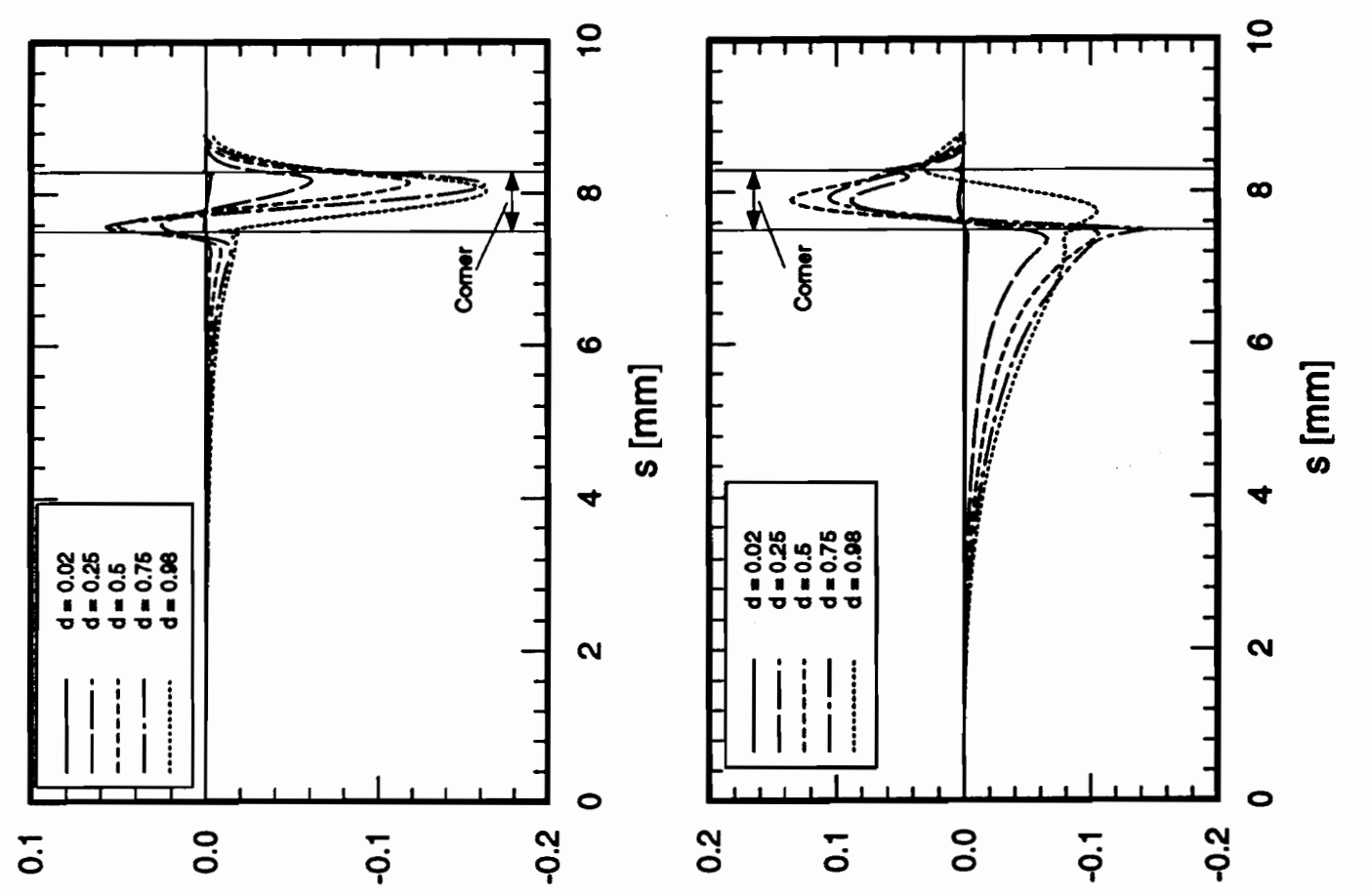

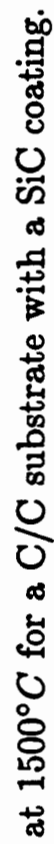

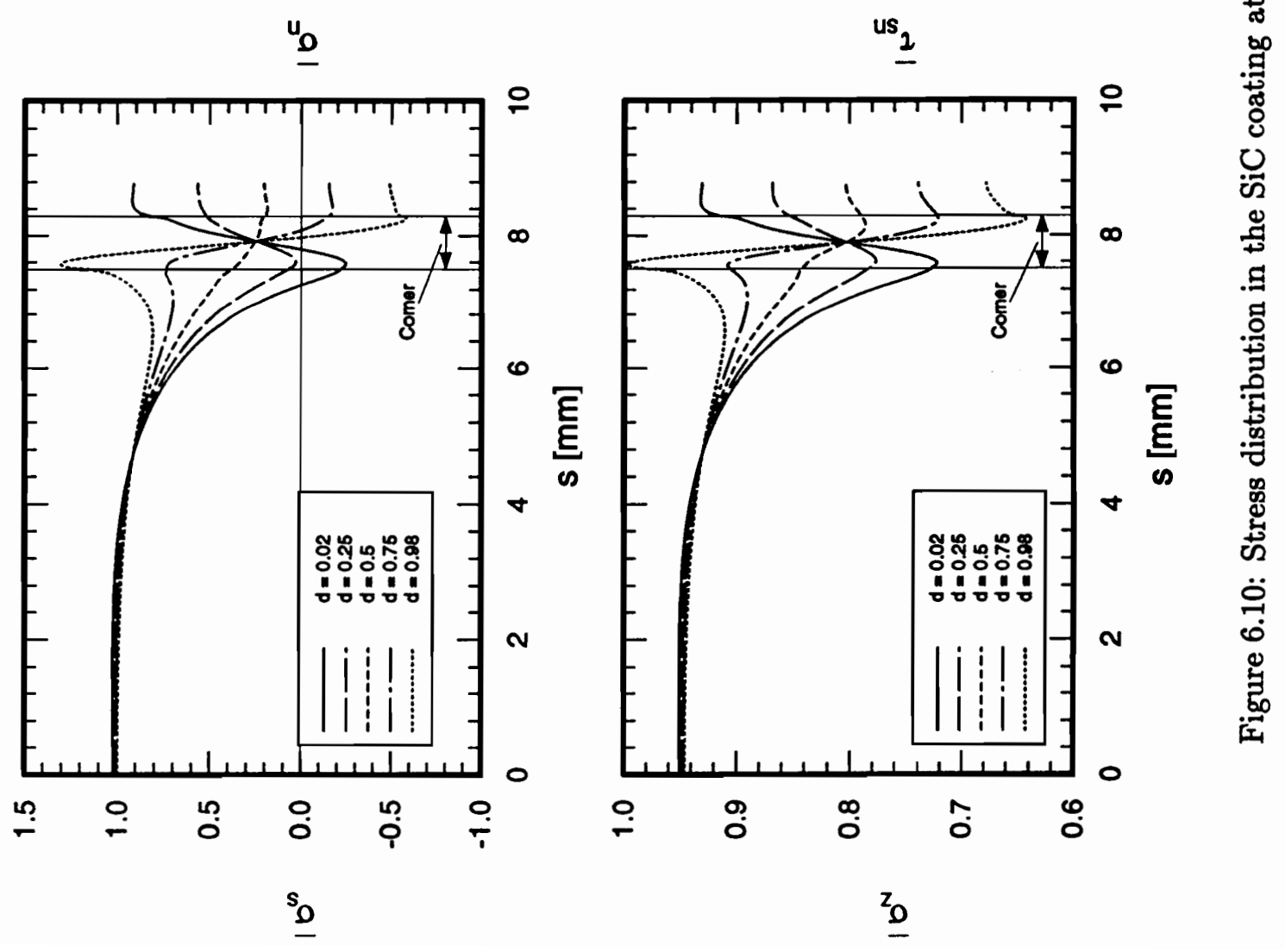




\subsection{Compensating/Compliant Coating Concept for Stress Reduction in Coatings}

In this section it is studied whether the stresses in the $\mathrm{SiC}$ coating can be reduced by inserting a compensating/compliant layer between the $\mathrm{C} / \mathrm{C}$ substrate and the $\mathrm{SiC}$ coating. The total coating thickness was kept constant so that stresses are not changed due to additional coating material. Keeping the total coating thickness constant also has practical significance, because $\mathrm{C} / \mathrm{C}$ composites are often used in weight-sensitive applications, and additional coating material might increase the weight of the structure.

First the effect of inserting a compliant pyrolytic carbon coating between the substrate and the coating is studied in Section 6.4.1. The influence of the SiC-pyrolytic carbon thickness ratio on the stresses is investigated. Next, in Sections 6.4.2 and 6.4.3, the influence of the coefficient of thermal expansion and the modulus of elasticity of the compensating/compliant layer on the stresses in the $\mathrm{SiC}$ coating are discussed. For this, the properties in the compliant coating were varied from those of the pyrolytic carbon coating. In Section 6.4.4 the effect of orthotropic compliant coatings is studied. Again, both the coefficient of thermal expansion and the moduli of elasticity were varied. Finally in Section 6.4.5 the results from the material property studies in Sections 6.4.2 - 6.4.4 are summarized.

\subsubsection{Influence of Thickness of a Pyrolytic Carbon Coating on Stresses}

In this parametric study it was investigated if inserting a soft or compliant pyrolytic carbon coating between the substrate and the coating could be used to reduce stresses in the coating. For this purpose an increasing fraction of the $\mathrm{SiC}$ coating was replaced by pyrolytic carbon. The material properties for pyrolytic carbon are given in Appendix A Stresses were calculated a $20^{\circ} \mathrm{C}$.

The stress components of interest for this compliant layer study were the normalized maximum tangential stress $\bar{\sigma}_{s(\max )}$, the normalized maximum normal stress $\bar{\sigma}_{n(\max )}$, the normalized maximum inplane shear stress $\bar{\tau}_{s n(\max )}$, and the normalized maximum axial 
stress $\bar{\sigma}_{z(\max )}$ in the coatings for different ratios of the pyrolytic carbon layer thickness to total coating thickness. These stresses were obtained by calculating the stresses in the $s-n-z$ coordinate system and then scanning the data to find the maximum absolute values of the stress components. Further, the maximum normalized principal material direction stresses in the substrate and the maximum principal stresses in the coatings were calculated. The principal material direction stress in the $\mathrm{C} / \mathrm{C}$ substrate in the direction of the fibers parallel to the $x$-direction in Fig. 5.1 is $\bar{\sigma}_{1}$, the stress in the direction perpendicular to the fibers, the $y$-direction, is $\bar{\sigma}_{2}$, and the stress in the direction of the axial fibers, or the $z$-direction, is $\bar{\sigma}_{z}$. The maximum principal stresses $\sigma_{I(\max )}$ and $\sigma_{I I(\max )}$ are the algebraically largest and smallest principal stresses in the coatings, respectively. The principal stress in the axial direction in the coatings and the substrate is identical to $\bar{\sigma}_{z}$. The maximum shear stress is the shear stress with the largest absolute value. The maximum values of all these stress components are shown in Table 6.2 for the different ratios of the pyrolytic carbon considered. (Be reminded that the stresses in Table 6.2 in the $\mathrm{SiC}$ and the pyrolytic carbon coating are the actual stresses normalized by the stresses in the $\mathrm{SiC}$ at $20^{\circ} \mathrm{C}$ for an infinitely wide substrate shown in Table 6.1 , and the stresses in the $\mathrm{C} / \mathrm{C}$ substrate are the actual stresses normalized by the stress in the substrate at $20^{\circ} \mathrm{C}$ shown in Table 6.1.) Depending on the stress component and the thickness of the pyrolytic carbon coating, each entry in Table 6.2 may occur at different location in the composite. The first column in the table corresponds to the baseline case discussed in Section 6.3. The tangential stress $\bar{\sigma}_{s}$ near the $\mathrm{SiC}$-pyrolytic carbon interface and the normal stress $\bar{\sigma}_{n}$ in the $\mathrm{SiC}$ coating at the locations of the maximum normal stress are shown in Fig. 6.11 as a function of the $s$-coordinate.

First consider the tangential stress $\bar{\sigma}_{s}$ in the SiC coating. In Table 6.2 it can be seen that the tangential stress $\bar{\sigma}_{\boldsymbol{s}(\max )}$ in the $\mathrm{SiC}$ coating decreases for increasing thickness of the pyrolytic carbon layer until it reaches a minimum at $t_{c a r b o n} / t_{t o t a l}=1 / 3$. Then the maximum tangential stress increases again for larger fractions of pyrolytic carbon. In Fig. 6.11 it can be seen that the maximum of the tangential stress is in the corner region for low pyrolytic carbon fractions. For high pyrolytic carbon fractions, the maximum is in the far field at the 


\begin{tabular}{|c|c|c|c|c|c|c|}
\hline & $\frac{+\infty}{m}$ & 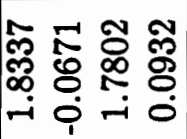 & 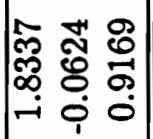 & 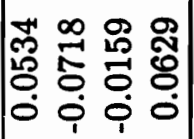 & $\left|\begin{array}{lll}\not{D} & 2 & 0 \\
0 & 0 & 0 \\
0 & 0 & 0 \\
0 & 0 & 0 \\
0 & 0 & 0\end{array}\right|$ & 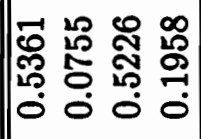 \\
\hline & $\mathbf{\sim}$ & 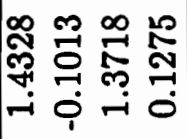 & 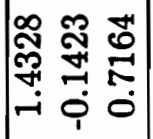 & 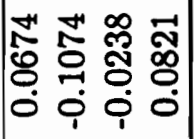 & 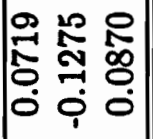 & 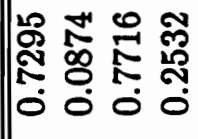 \\
\hline & $\stackrel{\sim}{-10}$ & 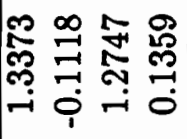 & 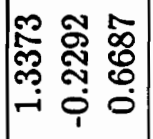 & 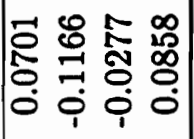 & 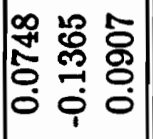 & 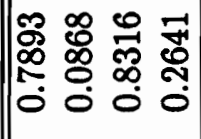 \\
\hline 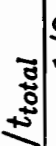 & $\stackrel{\infty}{-1}$ & 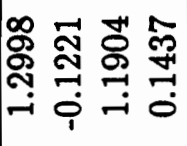 & ঙ્ઞ & 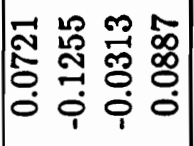 & 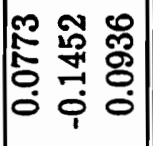 & 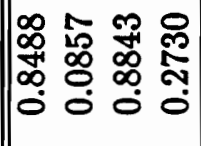 \\
\hline | & $\stackrel{+}{-}$ & 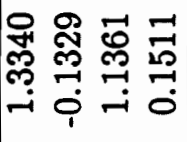 & 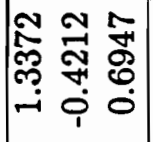 & 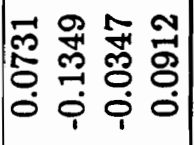 & 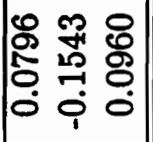 & 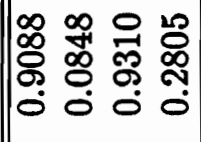 \\
\hline & $\stackrel{\infty}{-1}$ & 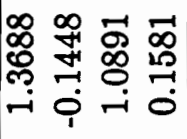 & 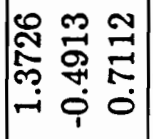 & 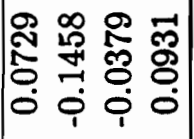 & 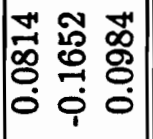 & 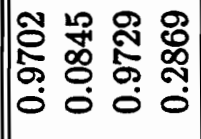 \\
\hline & $\stackrel{\sim}{=}$ & 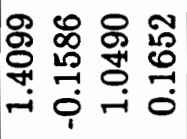 & 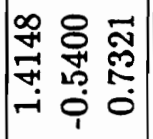 & 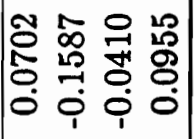 & $\left|\begin{array}{lll}0 & 5 & 0 \\
0 & 0 & 0 \\
0 & 0 & 0 \\
0 & 0 & 0 \\
0 & 0 & 0 \\
0\end{array}\right|$ & 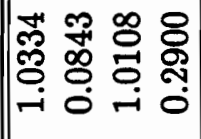 \\
\hline & 0 & 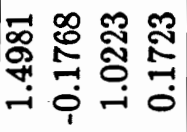 & 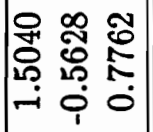 & $\begin{array}{llll}1 & 1 & 1 & 1\end{array}$ & 11 & 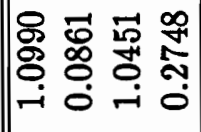 \\
\hline & 总 & 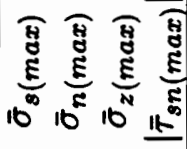 & 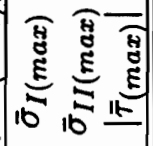 & 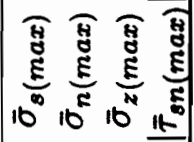 & 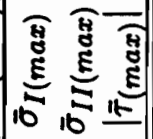 & 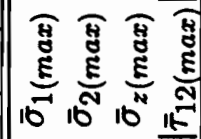 \\
\hline & : & نn & & ర̃: & & 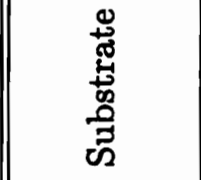 \\
\hline
\end{tabular}



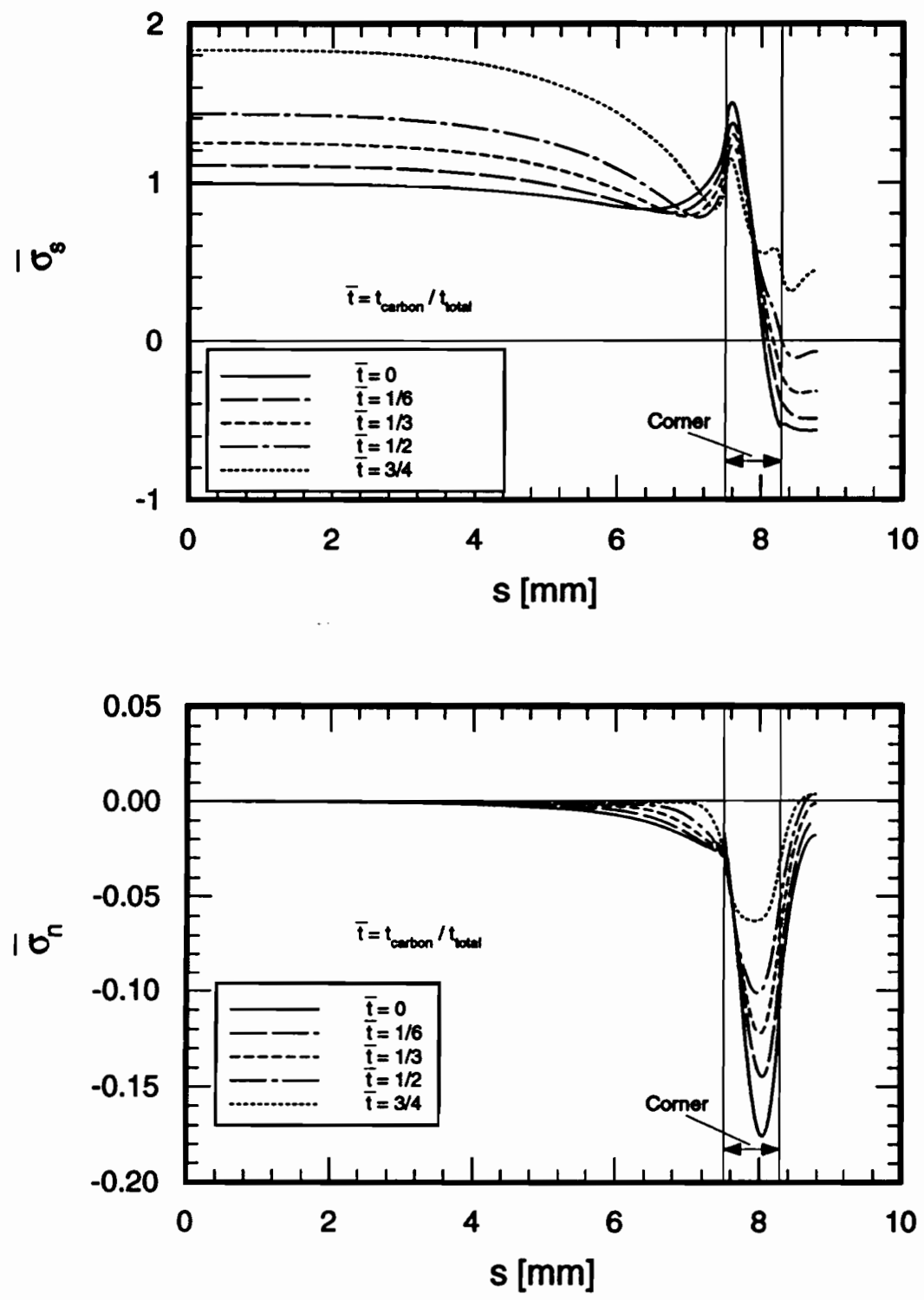

Figure 6.11: Tangential and normal stresses in SiC coating for different thicknesses of the pyrolytic carbon coating 
vertical line of symmetry of the composite. The magnitude of the stress concentration in the corner region decreases with increasing pyrolytic carbon thickness, but the magnitude of the far field tangential stress increases with increasing pyrolytic carbon thickness. The far field stress increases for increasing pyrolytic carbon thickness because there is less and less stiff material $(\mathrm{SiC})$ that can resist the thermal expansion of the substrate. As shown, a pyrolytic carbon thickness exists where the magnitude of the tangential stress at the vertical line of symmetry equals the maximum stress in the stress concentration in the corner region. At this pyrolytic carbon thickness, the maximum tangential stress $\bar{\sigma}_{s}$ reaches its smallest value.

As shown in Table 6.2, the axial stress $\bar{\sigma}_{z}$ in the $\mathrm{SiC}$ coating increases with increasing pyrolytic carbon thickness. However, its magnitude is always less than the magnitude of the maximum tangential stress. Therefore, the maximum tangential stress would be the failure-causing stress, and a decrease in this stress component due to the adding of a pyrolytic carbon layer is more important than the increase in $\bar{\sigma}_{z}$. The maximum axial stress occurs always near the vertical line of symmetry.

From Table 6.2 , it is seen that the maximum normal stress $\bar{\sigma}_{n(\max )}$ and maximum shear stress $\overline{\boldsymbol{\tau}}_{\boldsymbol{s n}(\max )}$ in the $\mathrm{SiC}$ coating both decrease with increasing pyrolytic carbon coating thickness. In Fig. 6.11 it can be seen that the maximum normal stress is always in the corner region.

The algebraically largest maximum principal stress $\sigma_{I(\max )}$ in the $\mathrm{SiC}$ coating follows the same trend as the maximum tangential stress. The difference in the magnitude between these two stresses is negligible. In fact, for thicknesses of the pyrolytic carbon layer for which the maximum stress occurs in the far field, the maximum principal stress and the maximum tangential stress coincide. This is the case because for these thicknesses the maximum stress occurs near the vertical line of symmetry, where the normal and shear stresses are zero. The algebraically smallest principal stress decreases in magnitude for increasing pyrolytic carbon coating thickness. As discussed for the maximum shear stress in Section 6.3, the maximum shear stress in the $\mathrm{SiC}$ coating is about $1 / 2$ the maximum 
principal stress.

Now consider the pyrolytic carbon ccating. The maximum tangential stress in this coating increases with increasing pyrolytic carbon thickness, reaches a maximum, and then begins decreasing again. The normal, axial, and shear stresses in this coating all decrease with increasing pyrolytic carbon thickness. At $t_{\text {carbon }} / t_{\text {total }}=1 / 3$, where the smallest maximum tangential stress in the $\mathrm{SiC}$ coating was observed, the tangential stress in the pyrolytic carbon coating reaches only about $7.2 \%$ of the baseline value. The normal stress and the shear stress reach $12.5 \%$ and $8.9 \%$ of the baseline stress, respectively. Therefore, because the normal stress is compressive, and thus delamination is not likely to occur, shear failure is the most likely cause of failure in the pyrolytic carbon coating. As it is for the $\mathrm{SiC}$ coating, the algebraically largest maximum principal stress in the pyrolytic carbon coating is close to the maximum tangential stress in this coating.

In the substrate the maximum principal material direction stresses $\bar{\sigma}_{1(\max )}, \bar{\sigma}_{z(\max )}$, and $\bar{\tau}_{12(\max )}$ all improve with increasing pyrolytic carbon thickness. The stress $\sigma_{2(\max )}$ remains fairly constant for a wide range of pyrolytic carbon thicknesses. It only begins to decrease for large fractions of pyrolytic carbon.

The undeformed finite element mesh for the substrate and coatings is shown in Fig. 6.12a. The deformed meshes for the substrate with only a SiC coating and for the substrate with a pyrolytic carbon coating and $\mathrm{a} \mathrm{SiC}$ coating for $t_{\text {carbon }} / t_{\text {total }}=1 / 3$ are shown in Figs $6.12 \mathrm{~b}$ and c, respectively. The thickness of the pyrolytic carbon coating in the figure is close to the thickness for which the maximum tangential stress is a minimum. The soft pyrolytic carbon coating shows large shear deformations in the corner region. This large shear deformation is not present in the substrate with a pure SiC coating in Fig. 6.12b. From this observation it is apparent that the shear deformation in the pyrolytic carbon layer causes the stress relief in the $\mathrm{SiC}$ coating. Further, it can be seen that the shear deformation propagates several corner radii away from the corner. The large shear deformation in the pyrolytic carbon coating explains the relatively large maximum shear stresses in this coating. 

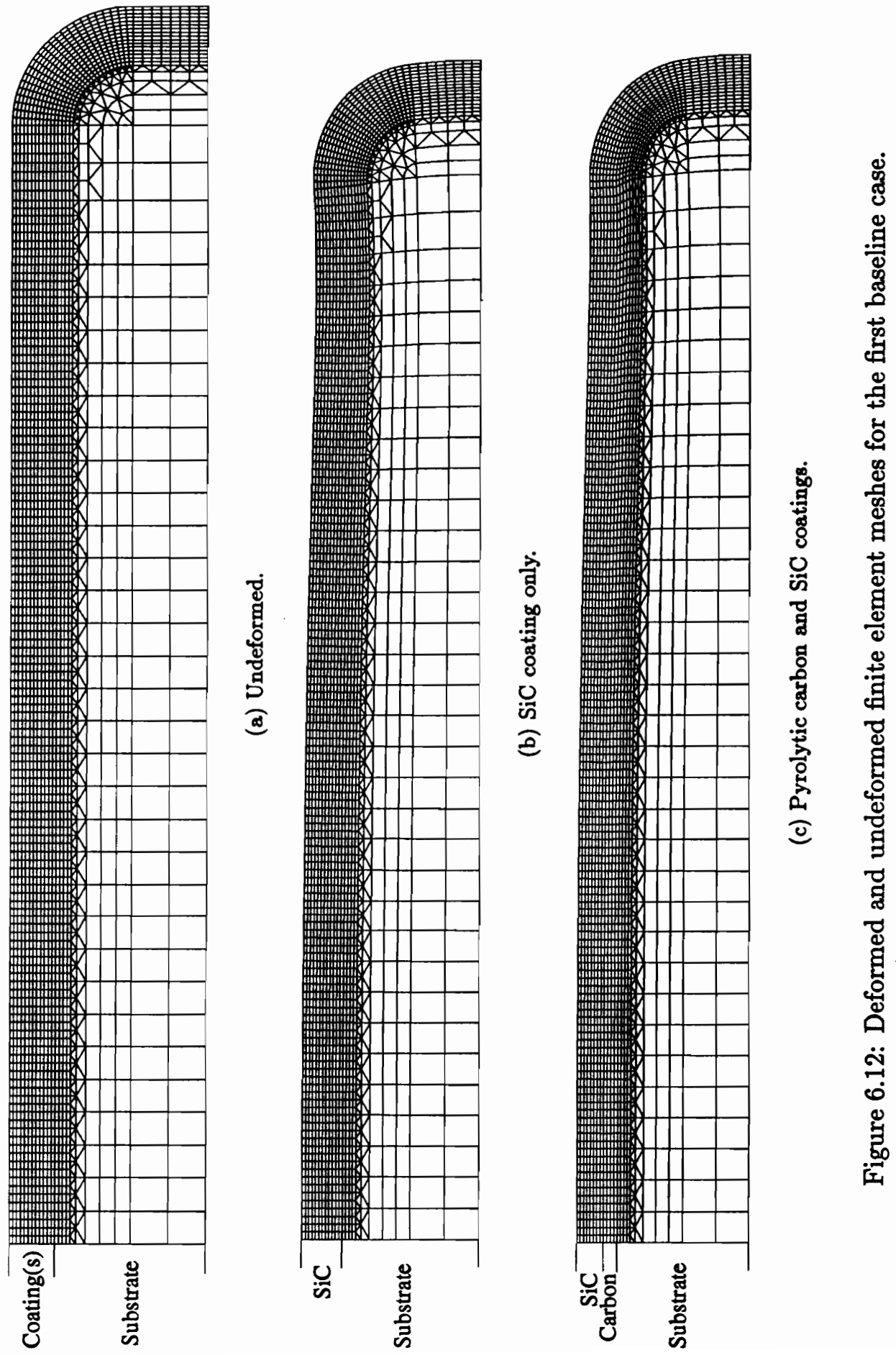


\subsubsection{Influence of the Coefficient of Thermal Expansion of the Compliant Coating on Stresses}

It was found in the last section that a thickness of the pyrolytic carbon coating exists at which the tangential stress in the $\mathrm{SiC}$ coating reaches a minimum. In this section results obtained from varying the coefficients of thermal expansion of the compliant coating from those of the pyrolytic carbon coating are discussed. The coefficients of thermal expansion of the compliant coating were varied for the $\mathrm{C} / \mathrm{C}$ substrate with a compliant coating and a $\mathrm{SiC}$ coating as described in the previous sections.

First consider the effect of varying the coefficient of thermal expansion for a thickness of the compliant coating of $t_{\text {compl. }} / t_{\text {total }}=1 / 3$. This thickness is close to the thickness that resulted in minimum tangential stresses for the variation of the thickness of the pyrolytic carbon coating for the material properties discussed in the last section. The maximum stresses in the coatings and in the substrate for different coefficients of thermal expansion of the compliant coating are shown for $t_{\text {compl. }} / t_{\text {total }}=1 / 3$ in Table 6.3. Because, as was discussed in Sections 6.3 and 6.4.1, the maximum principal stress and the maximum tangential stress are almost identical, only the stresses in the $s-n-z$ coordinate system will be shown for the coatings, and the principal material direction stresses will be shown for the substrate. In Table 6.3 it can be seen that in the SiC coating all stress components decrease with increasing coefficient of thermal expansion of the compliant coating. For low coefficients of thermal expansion, the maximum tangential stress $\bar{\sigma}_{s(\max )}$ decreases rapidly with increasing coefficient of thermal expansion and then for higher coefficients of thermal expansion $\bar{\sigma}_{s(\max )}$ decreases more slowly. The tangential stress in the $\mathrm{SiC}$ coating near the SiC-compliant coating interface, the location at which the stress concentration in the $\mathrm{SiC}$ coating is the largest, is shown for the different values of the coefficient of thermal expansion in Fig. 6.13a. As can be seen, both the far field value and the stress concentration value in the corner region decrease with increasing coefficient of thermal expansion. The decrease of the stress concentration is more rapid than the decrease of the far field stress. 
Therefore, for low coefficients of thermal expansion, for which the maximum stress occurs in the corner, the maximum tangential stress decreases faster than for high coefficients of thermal expansion, for which the maximum tangential stress is in the far field. The normal stress in the $\mathrm{SiC}$ coating near the SiC-compliant coating interface is shown in Fig. 6.13b. It can be seen that the normal stress, which is mostly compressive, is confined to the corner region for all coefficients of thermal expansion and it decreases with increasing coefficients of thermal expansion.

In the compliant coating, the maximum tangential stress $\bar{\sigma}_{s(\max )}$, the maximum axial stress $\bar{\sigma}_{z(\max )}$, and the maximum shear stress $\bar{\tau}_{s n(\max )}$ shown in Table 6.3 for $t_{\text {carbon }} / t_{\text {total }}=$ $1 / 3$ all increase with increasing coefficient of thermal expansion. In contrast, the maximum normal stress $\bar{\sigma}_{n(\max )}$ decreases in magnitude with increasing coefficient of thermal expansion. The magnitudes of the maximum axial and tangential stresses in the compliant coating are much smaller than in the $\mathrm{SiC}$ coating.

The maximum principal material direction stresses $\bar{\sigma}_{1(\max )}$ and $\bar{\sigma}_{z(\max )}$, and the maximum shear stress $\bar{\tau}_{12(\max )}$ in the substrate all increase with increasing coefficient of thermal expansion of the compliant coating. The maximum principal material direction stress $\bar{\sigma}_{2(\max )}$ decreases with increasing coefficient of thermal expansion. Due to the much smaller modulus of the substrate in the 2-direction, the stress $\bar{\sigma}_{2(\max )}$ is much smaller than the other stress components.

In Section 6.4.1 it was shown that for increasing thickness of the pyrolytic carbon coating for constant material properties, the far field tangential stress in the $\mathrm{SiC}$ coating increased slowly, while the stress concentrations in the $\mathrm{SiC}$ coating decreased rapidly. In this section it was shown that for an increasing coefficient of thermal expansion of the compliant coating for a fixed thickness of $t_{\text {compl. }} / t_{\text {total }}=1 / 3$, both the far field tangential stress and the stress concentration in the corner region in the $\mathrm{SiC}$ coating decreased. To investigate if there is a combination of coefficient of thermal expansion and thickness of the compliant coating for which the maximum stresses in the $\mathrm{SiC}$ coating would have their smallest values, the combined effect of varying both the compliant coating thickness and the coefficient of 


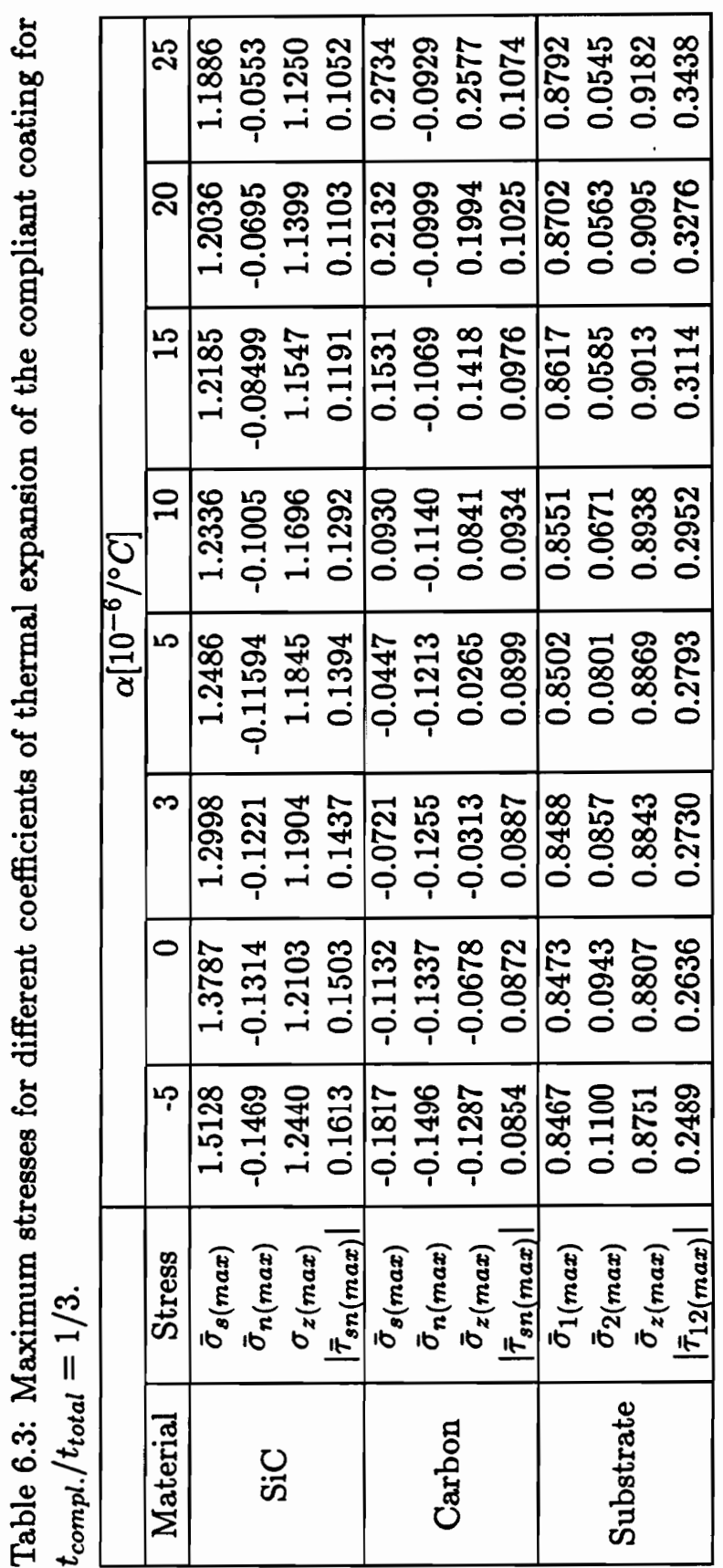




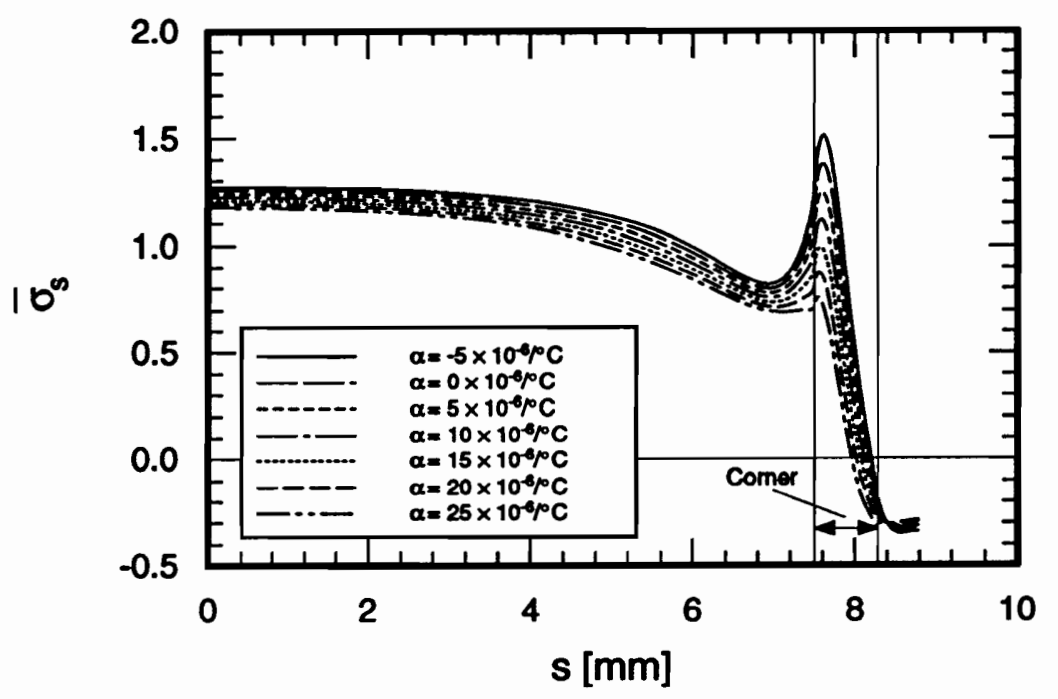

(a) Tangential stress.

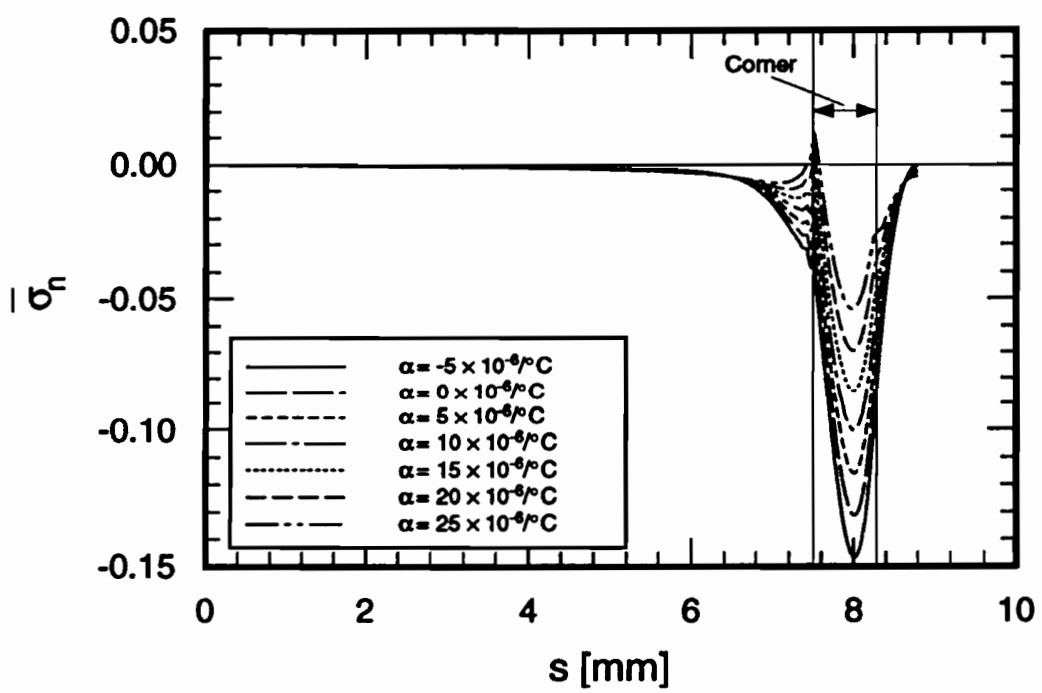

(b) Normal stress.

Figure 6.13: Tangential stress and normal stress in SiC coating for different coefficients of thermal expansion of the compliant coating. 
thermal expansion of the compliant coating on the maximum stresses was investigated. For this purpose the thickness of the compliant coating was varied form $t_{\text {carbon }} / t_{\text {total }}=0$ to $t_{\text {carbon }} / t_{\text {total }}=1 / 2$ and the coefficient of thermal expansion of the compliant coating was varied from 0 to $25 \times 10^{-6} /{ }^{\circ} \mathrm{C}$. For a number of combinations of thickness and coefficient of thermal expansion of the compliant coating, the maxima of the stress components in the coatings and the substrate were calculated. Contour graphs of these maxima as functions of the thickness and the coefficient of thermal expansion of the pyrolytic carbon coating were prepared. It should be noted that the maxima of the stresses shown do not necessarily occur at the same locations in the composite.

The maximum stresses in the $\mathrm{SiC}$ coating as a function of the thickness and the coefficient of thermal expansion of the compliant coating are shown in the contour graphs in Fig. 6.14. First focus on the maximum tangential stress in Fig. 6.14a on page 117. It can be seen that the smallest maximum tangential stress occurs for a compliant coating thickness of $t_{\text {compl. }} / t_{\text {total }}=1 / 6$ and for a coefficient of thermal expansion of $25 \times 10^{-6} /{ }^{\circ} \mathrm{C}$. For decreasing coefficients of thermal expansion, the smallest maximum tangential stress shifts towards larger compliant coating thicknesses. In Fig. 6.14b it is shown that the maximum normal stress decreases with increasing compliant coating thickness and with increasing coefficient of thermal expansion. The maximum axial stress shown in Fig. 6.14c is smallest for small $t_{\text {compl. }} / t_{\text {total }}$ ratios. This stress component is somewhat insensitive to changes in the coefficient of thermal expansion. The maximum shear stress shown in Fig. 6.14d varies similarly to the normal stress. However it does not change as rapidly as the normal stress with either parameter. These observations show that choosing the right combination of coefficient of thermal expansion and thickness of the compliant coating can result in significantly reduced stresses in the $\mathrm{SiC}$ coating compared to the baseline case. For higher coefficients of thermal expansion of the compliant coating, lower thicknesses of this coating are necessary to achieve lower stresses.

The variation of the maximum normalized stresses in the compliant coating with both the coefficient of thermal expansion and the thickness of the compliant coating are shown in 
Fig. 6.15. The maximum tangential stress and the maximum axial stress are both insensitive to the thickness of the compliant coating. Both stresses increase with increasing coefficient of thermal expansion. However, their maximum magnitude is much smaller than the stresses in the $\mathrm{SiC}$ coating. The maximum normal stress decreases in magnitude with increases in both the coefficient of thermal expansion and the thickness. It is always compressive. In contrast to the maximum normal stress, the maximum shear stress increases with the coefficient of thermal expansion for all thicknesses of the compliant coating. For high values of the coefficient of thermal expansion, it reaches a maximum value at about $t_{\text {compl. }} / t_{\text {total }}=$ 1/6. This location of the largest maximum shear stress in the compliant coating in the coefficient of thermal expansion-thickness parameter space coincides with the location of the smallest tangential stress in the $\mathrm{SiC}$ coating in the coefficient of thermal expansion-thickness parameter space. This supports the conclusions drawn in Section 6.4.1 that the shearing of the compliant coating plays an important role in reducing the stress concentrations in the $\mathrm{SiC}$ coating (see Fig. 6.12c).

The normalized maximum principal material direction stresses $\bar{\sigma}_{1}$ and $\bar{\sigma}_{z}$ in the substrate, shown in Fig. 6.16, are insensitive to changes of the coefficient of thermal expansion of the compliant coating. However, they decrease with increasing thickness of the compliant coating. The transverse principal material direction stress $\bar{\sigma}_{2}$ decreases with increasing compliant coating coefficient of thermal expansion and increasing thickness. The maximum shear stress $\bar{\tau}_{12}$ in the substrate has a maximum value at the same location in the parameter space as the maximum shear stress in the compliant coating.

In this section it has been shown that the stresses in the $\mathrm{SiC}$ coating can be reduced significantly if the right combination of thickness and coefficient of thermal expansion of the compliant coating is chosen. This reduction of stresses in the $\mathrm{SiC}$ coating was achieved without significantly increasing the stresses in the compliant coating and the substrate. 


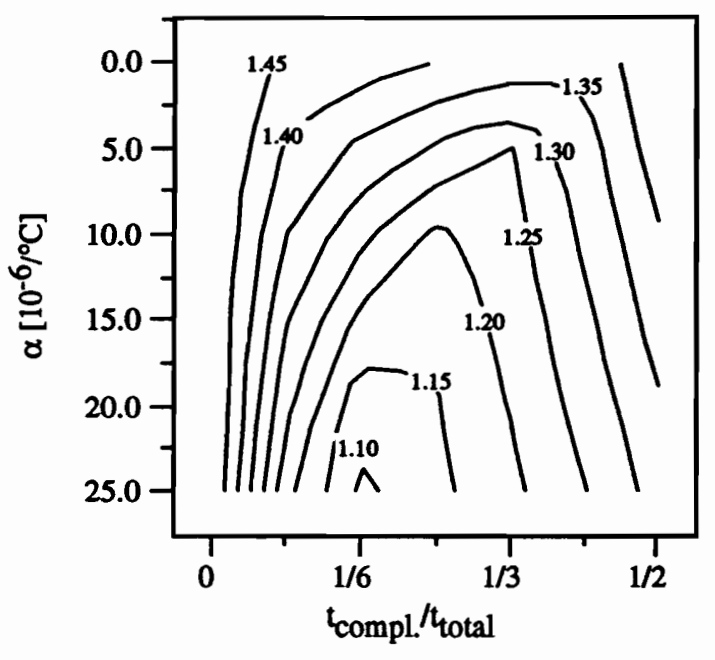

(a) $\bar{\sigma}_{s(\max )}$

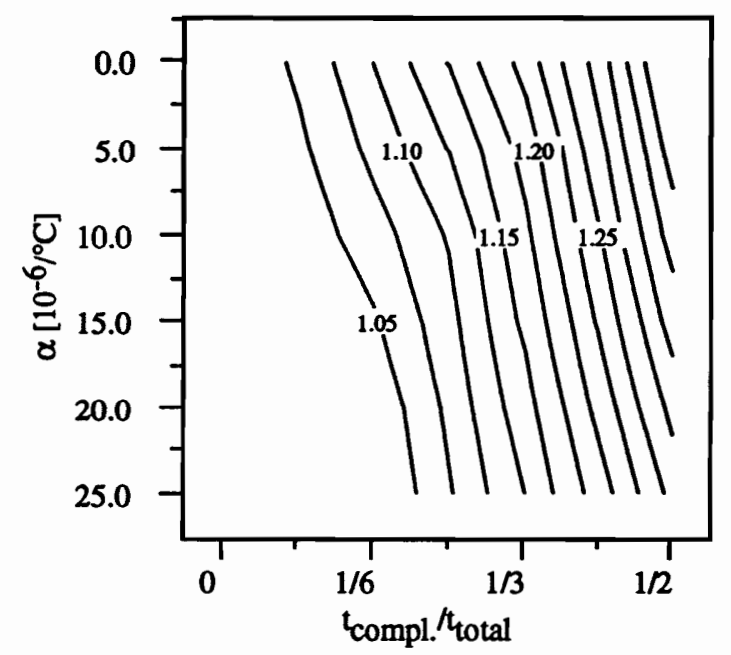

(c) $\bar{\sigma}_{z(\max )}$

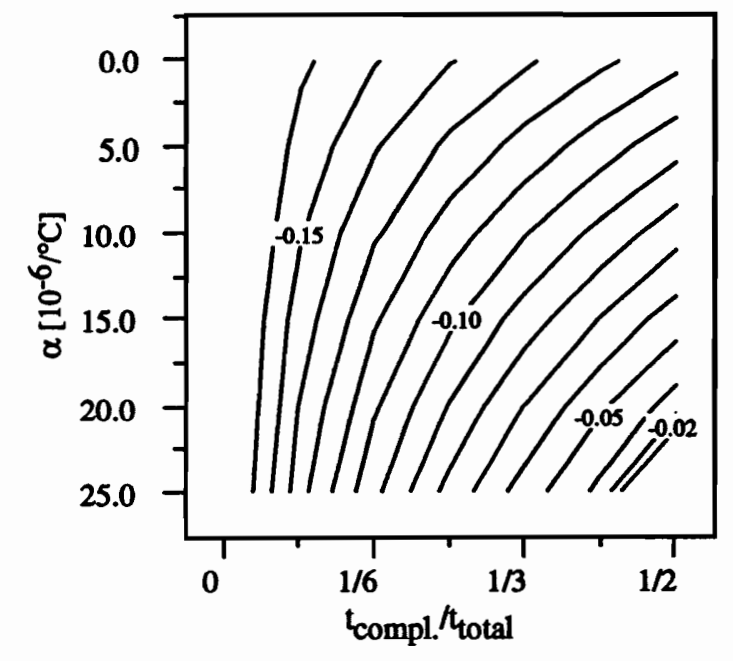

(b) $\bar{\sigma}_{n(\max )}$

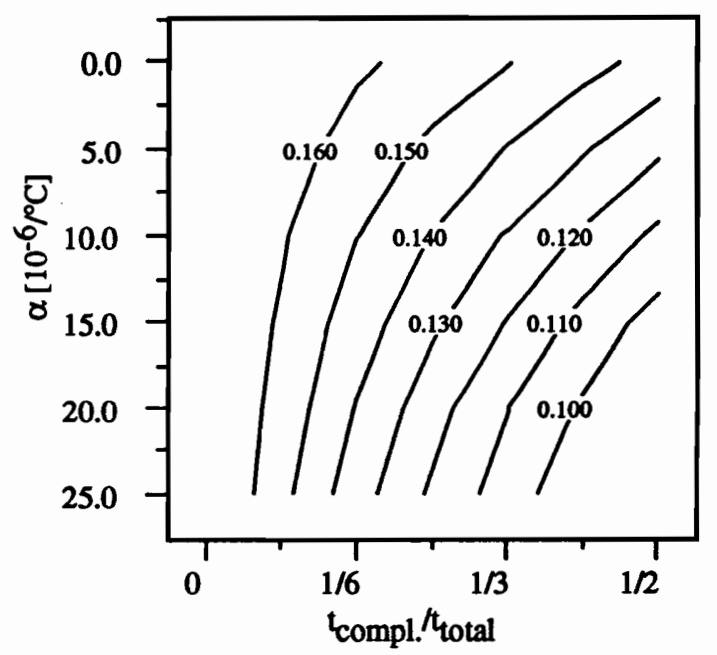

(d) $\bar{\tau}_{\operatorname{sn}(\max )}$

Figure 6.14: Effect of thickness and coefficient of thermal expansion of the compliant coating on the maximum stresses in the $\mathrm{SiC}$ coating. 


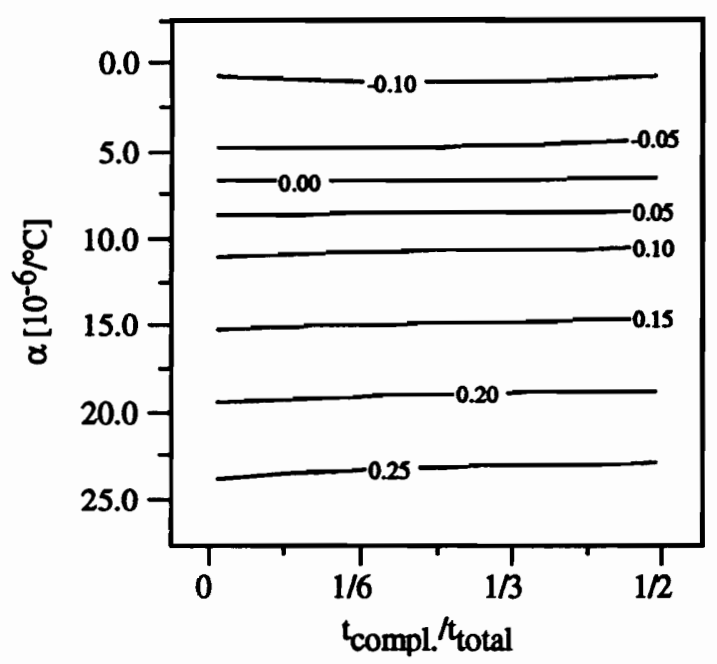

(a) $\bar{\sigma}_{\text {e(max })}$

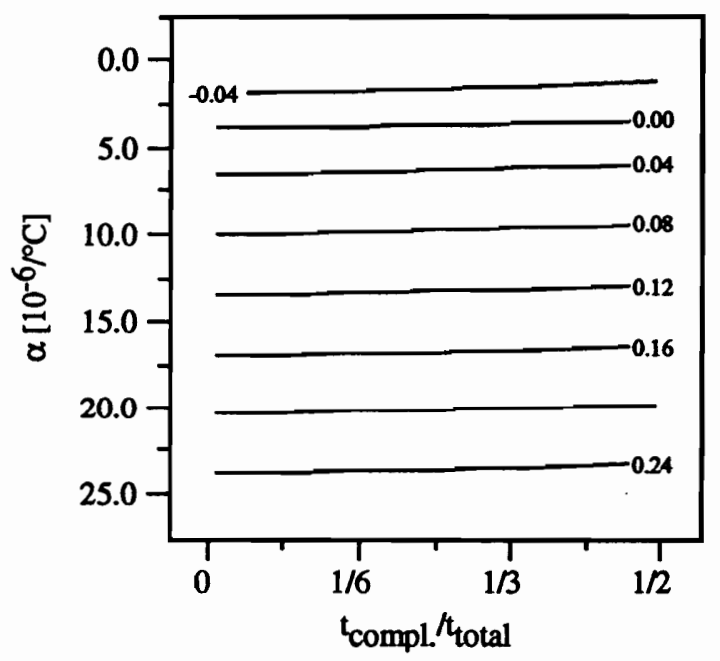

(c) $\bar{\sigma}_{x(\max )}$

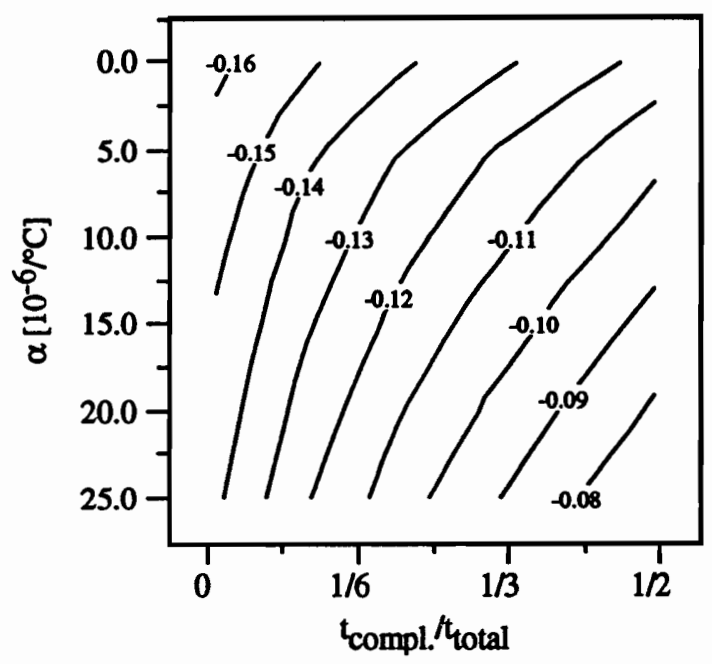

(b) $\bar{\sigma}_{n(\max )}$

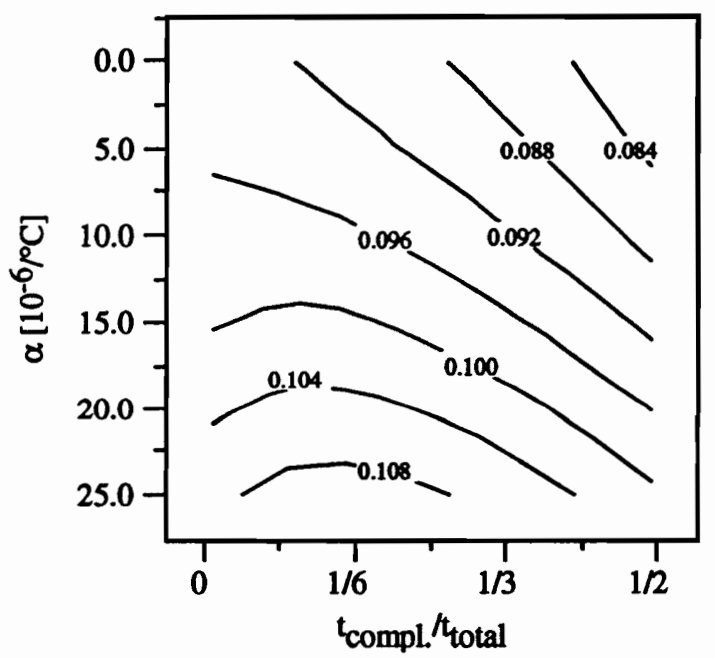

(d) $\bar{\tau}_{\operatorname{sn}(\max )}$

Figure 6.15: Effect of thickness and coefficient of thermal expansion of the compliant coating on the maximum stresses in the compliant coating. 


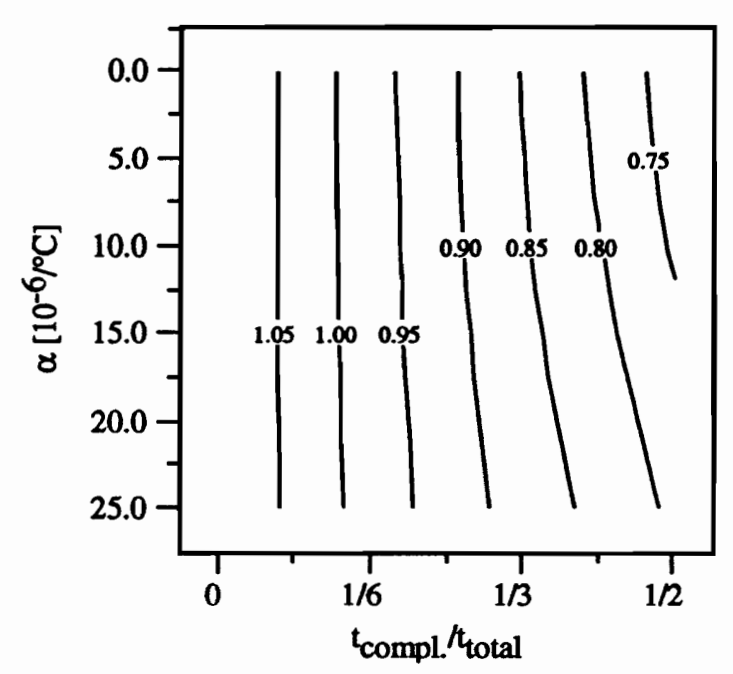

(a) $\bar{\sigma}_{1(\max )}$

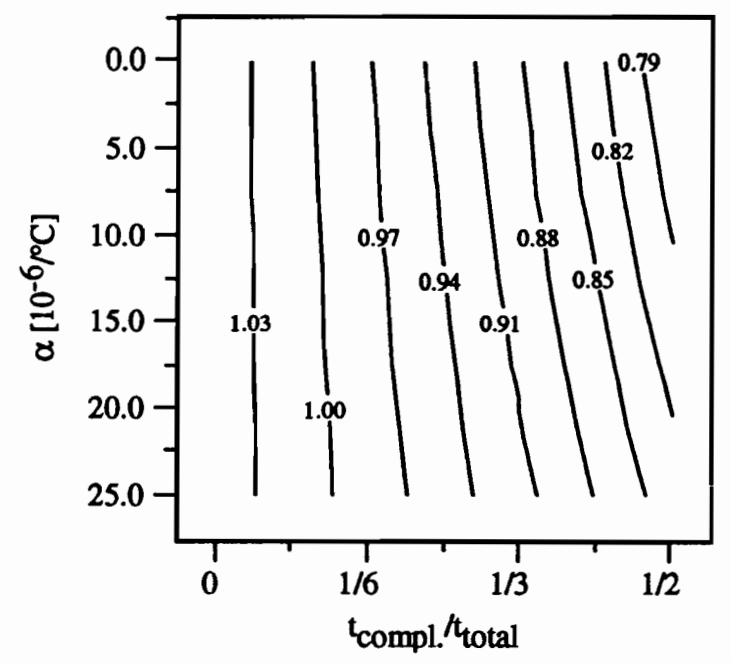

(c) $\bar{\sigma}_{z(\max )}$

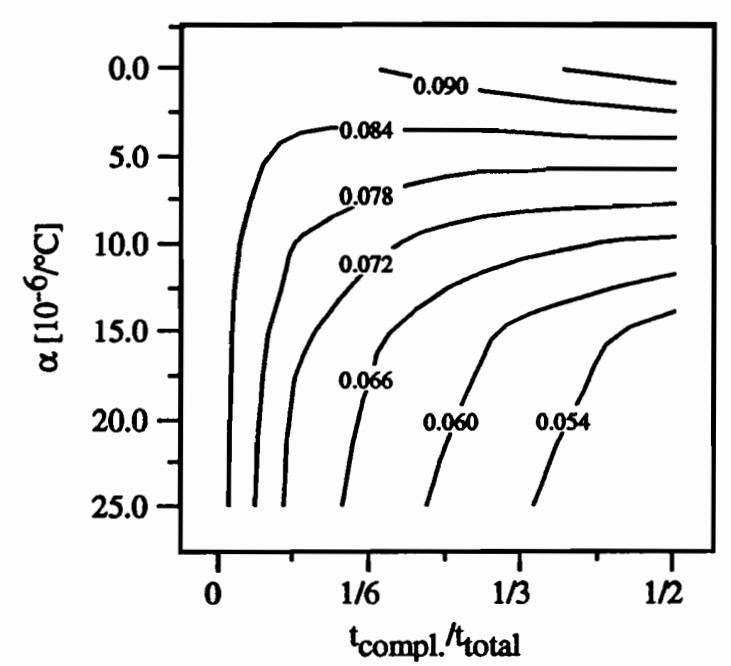

(b) $\bar{\sigma}_{2(\max )}$

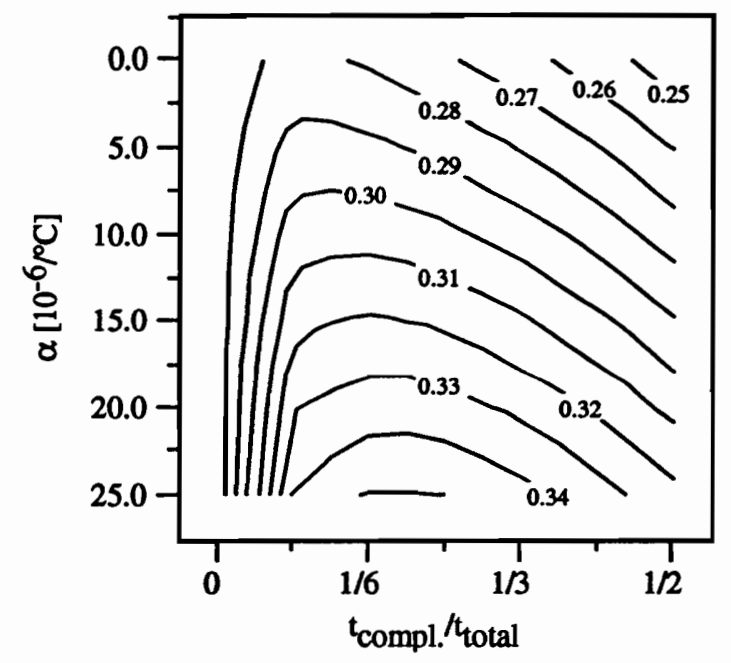

(d) $\bar{\tau}_{12(\max )}$

Figure 6.16: Effect of thickness and coefficient of thermal expansion of the compliant coating on the maximum stresses in the $\mathrm{C} / \mathrm{C}$ substrate. 


\subsubsection{Influence of the Modulus of Elasticity of the Compliant Coating on Stresses in the Coatings}

In the last section it was shown that increasing the coefficient of thermal expansion of the compliant coating resulted in lower stresses in the $\mathrm{SiC}$ coating. The effect of changing the modulus of elasticity of the compliant coating is discussed in this section. As was done for the variation of the coefficient of thermal expansion in Section 6.4.2, the combined effect of varying the modulus of elasticity and the thickness of the compliant coating was analyzed. The modulus of elasticity of the compliant coating was varied from $10 \mathrm{GPa}$ to $150 \mathrm{GPa}$ and the Poisson's ratios were kept constant. Therefore, because isotropic coatings were considered, the shear modulus of the compliant coating varied proportional to the modulus of elasticity as $G=E /(2(1+\nu))$. The contour plots of the maxima of the stress components in the coatings and the substrate as a function of the modulus of elasticity and the thickness of the compliant coating are shown in Figs. 6.17 - 6.19. As was done for the cases discussed in the last section, the maximum stress components in the s-n-z coordinate system are shown for the coatings, and the maximum principal material direction stresses are shown for the substrate.

As can be seen in Fig. 6.17a, the maximum tangential stress $\bar{\sigma}_{s}$ in the $\mathrm{SiC}$ coating reaches a minimum value for approximately $t_{\text {compl. }} / t_{\text {total }}=1 / 4$, for any modulus of elasticity. For thicknesses close to $t_{\text {compl. }} / t_{\text {total }}=1 / 4$ a slight decrease of the tangential stress in the SiC coating can be observed for an increasing modulus of elasticity. For all other thicknesses, the maximum tangential stress is insensitive to changes in the modulus of elasticity. Both the maximum axial stress $\bar{\sigma}_{z}$ (Fig. 6.17c) and the maximum shear stress $\bar{\tau}_{s n}$ (Fig. 6.17d) in the $\mathrm{SiC}$ coating are insensitive to changes in the modulus of elasticity of the compliant coating. The axial stress increases with increasing thickness of the compliant coating, while the shear stress decreases with increasing thickness. The normal stress $\bar{\sigma}_{n}$ in the $\mathrm{SiC}$ coating (Fig. 6.17b) decreases with decreasing modulus and increasing thickness of the compliant coating. 
The variation of the stresses in the compliant coating shown in Fig. 6.18 is very different than for the $\mathrm{SiC}$ coating. The maximum tangential stress $\bar{\sigma}_{s}$ in the compliant coating (Fig. 6.18a) is insensitive to changes of the thickness, but its magnitude increases fivefold over the range of moduli of elasticity considered. Since for this specific case the stress is compressive, this increase may not cause any damage in the coating. However, for different coefficients of thermal expansion of the compliant coating the stress may be tensile, and then the increase of stress may cause failure in the compliant coating, before the outer $\mathrm{SiC}$ coating would fail. This will be discussed later in this section in more detail. The normal stress $\bar{\sigma}_{n}$ in the compliant coating (Fig. 6.18b) varies with thickness and modulus in the same way as the normal stress in the $\mathrm{SiC}$ coating. The axial stress $\bar{\sigma}_{z}$ (Fig. 6.18c) increases in magnitude with increasing modulus of elasticity for small and for large thicknesses of the compliant coating, while it is insensitive to changes in the modulus for intermediate thicknesses. For all moduli the axial stress increases with increasing thickness. The main trend in the shear stress $\bar{\tau}_{s n}$ (Fig. 6.18d) is a decrease with increasing thickness and decreasing modulus. The overall change of the shear stress within the parameter space is small.

In the substrate the maximum principal material direction stresses $\bar{\sigma}_{1}, \bar{\sigma}_{2}$, and $\bar{\sigma}_{z}$ shown in Figs. 6.19a-c are all, to a large degree, insensitive to changes in the modulus of the compliant coating, and all decrease with increasing thickness of the compliant coating. The maximum shear stress $\bar{\tau}_{12}$ in the substrate (Fig. 6.19d) has its largest value for a small modulus and an intermediate thickness of the compliant coating. The overall change in the stress components $\bar{\sigma}_{2}$ and $\bar{\tau}_{12}$ is small.

In the discussion of the maximum tangential stress in the compliant coating it was observed that the sign of the stress may depend on the coefficient of thermal expansion of the compliant coating (see Table 6.3 on page 113). To investigate this further, the combined effects of changing the modulus of elasticity and the coefficient of thermal expansion of the compliant coating were analyzed for an compliant coating thickness of $t_{\text {compl. }} / t_{\text {total }}=1 / 3$. The modulus of the compliant coating was varied from $10 \mathrm{GPa}$ to $150 \mathrm{GPa}$ and the coefficient of thermal expansion was varied from 0 to $10 \times 10^{-6} /{ }^{\circ} \mathrm{C}$. For several combinations of 


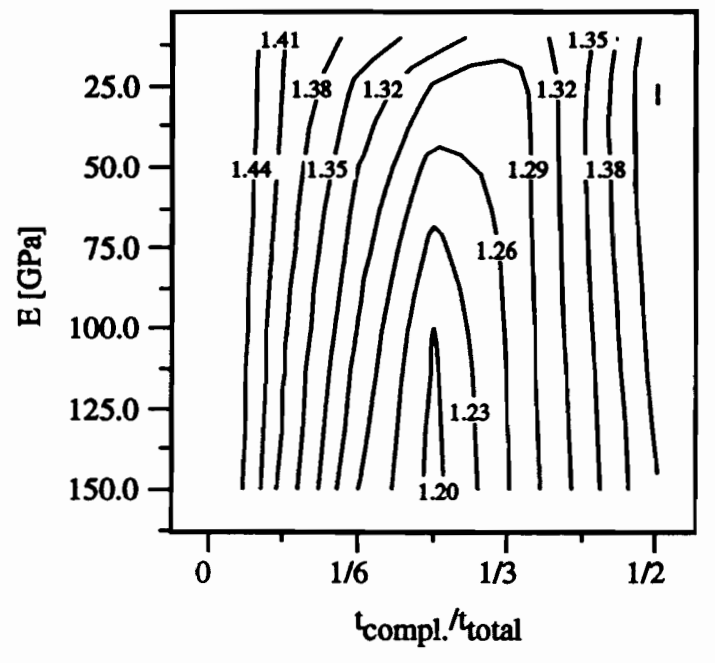

(a) $\bar{\sigma}_{s(m e x)}$

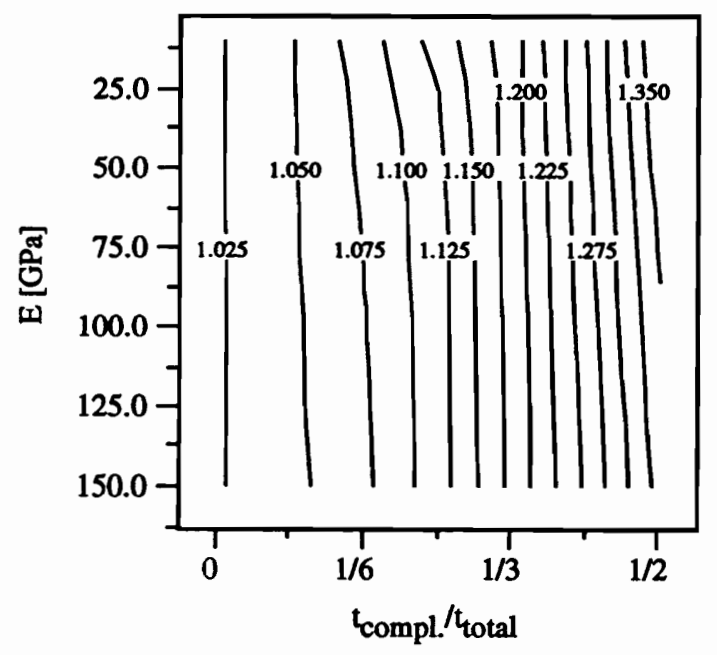

(c) $\bar{\sigma}_{x(\max )}$

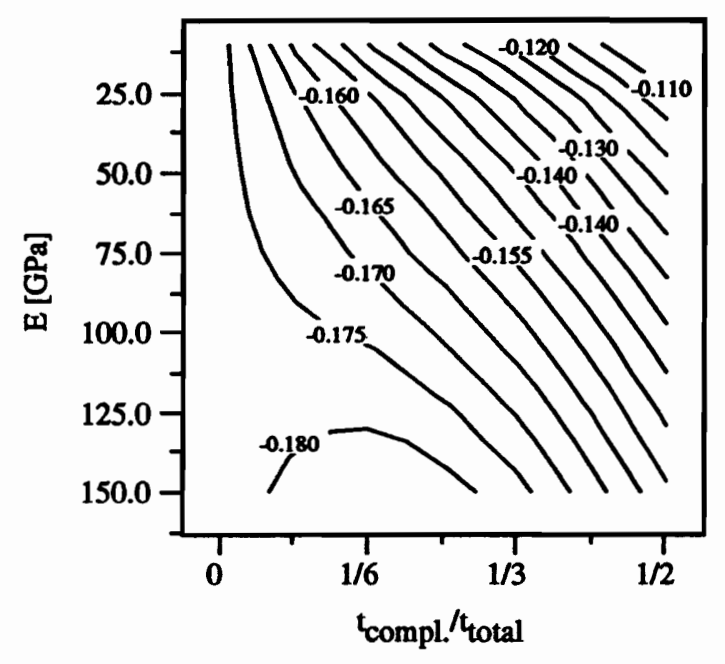

(b) $\bar{\sigma}_{n(\max )}$

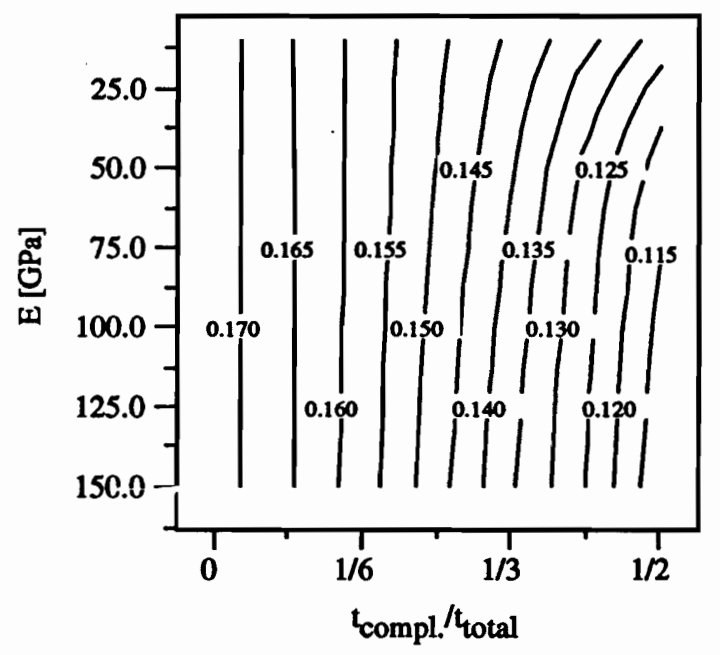

(d) $\bar{\tau}_{\operatorname{sn}(\max )}$

Figure 6.17: Effect of thickness and modulus of elasticity of the compliant coating on the maximum stresses in the $\mathrm{SiC}$ coating. 


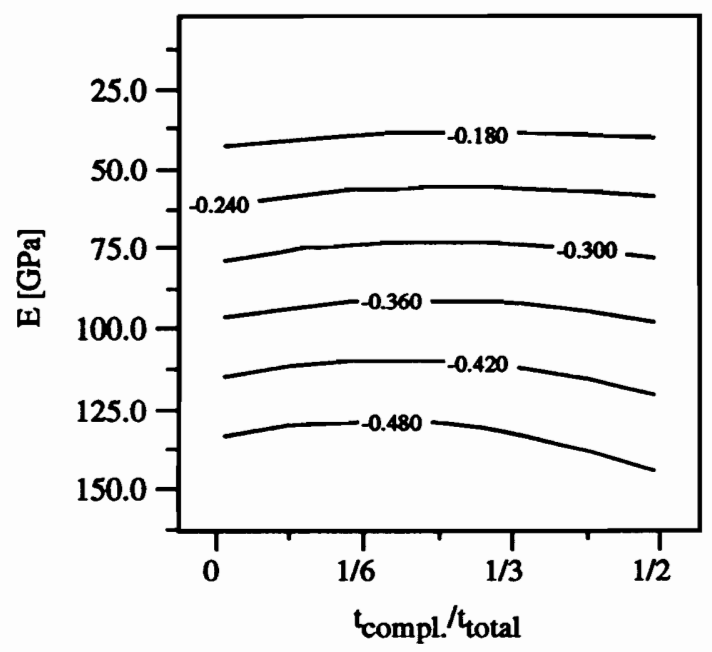

(a) $\bar{\sigma}_{s(\max )}$

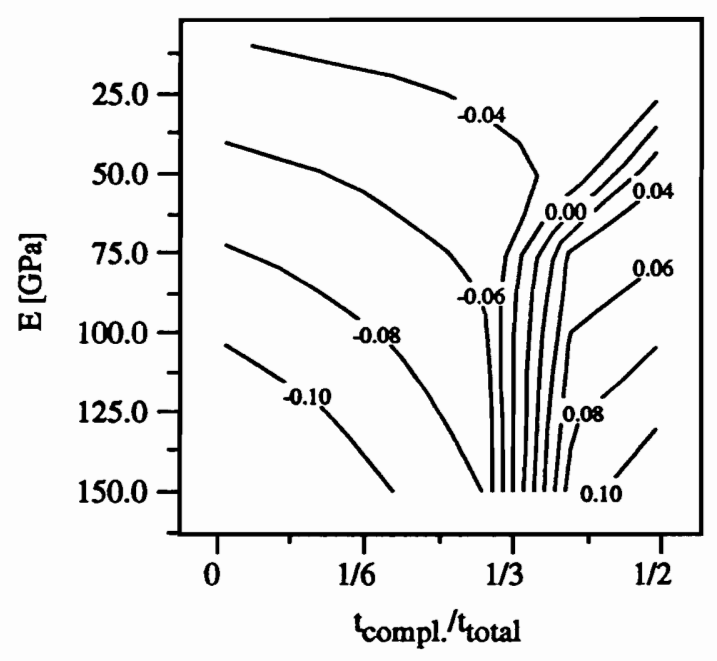

(c) $\bar{\sigma}_{x(\max )}$

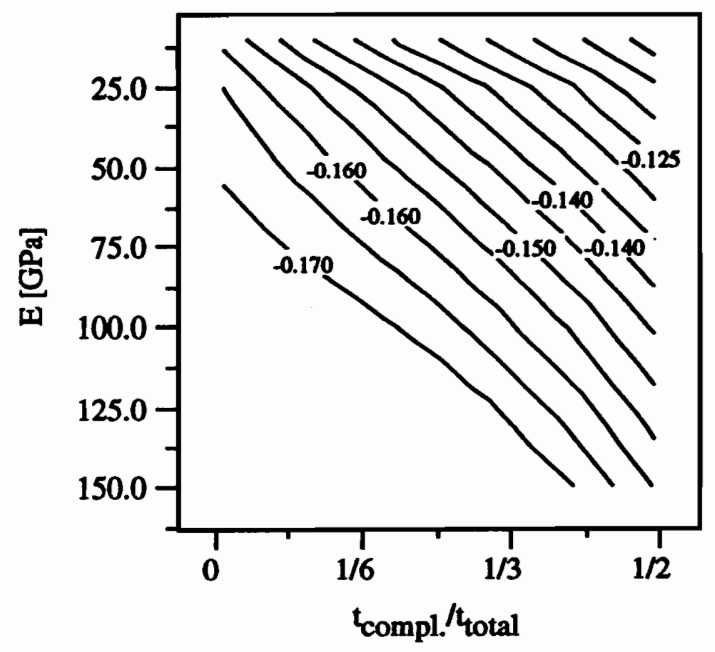

(b) $\bar{\sigma}_{n(m a x)}$

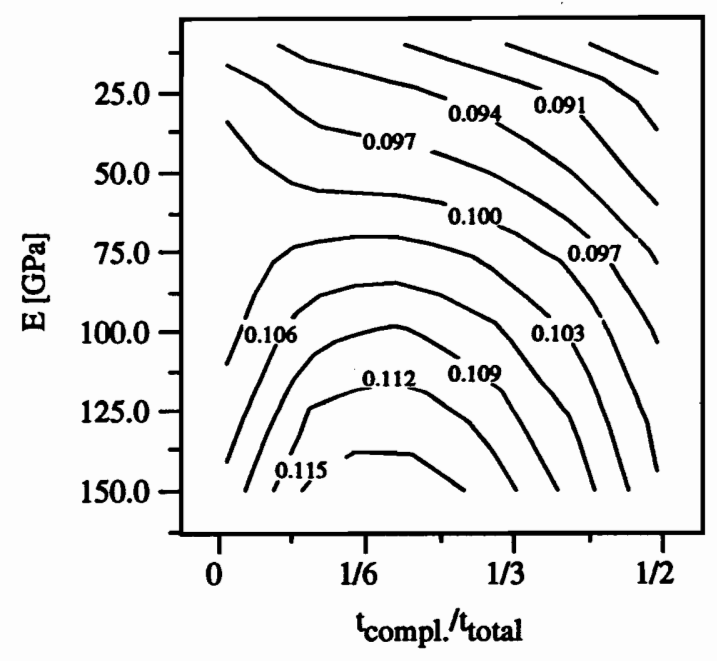

(d) $\bar{\tau}_{\text {on }(\max )}$

Figure 6.18: Effect of thickness and modulus of elasticity of the compliant coating on the maximum stresses in the compliant coating. 


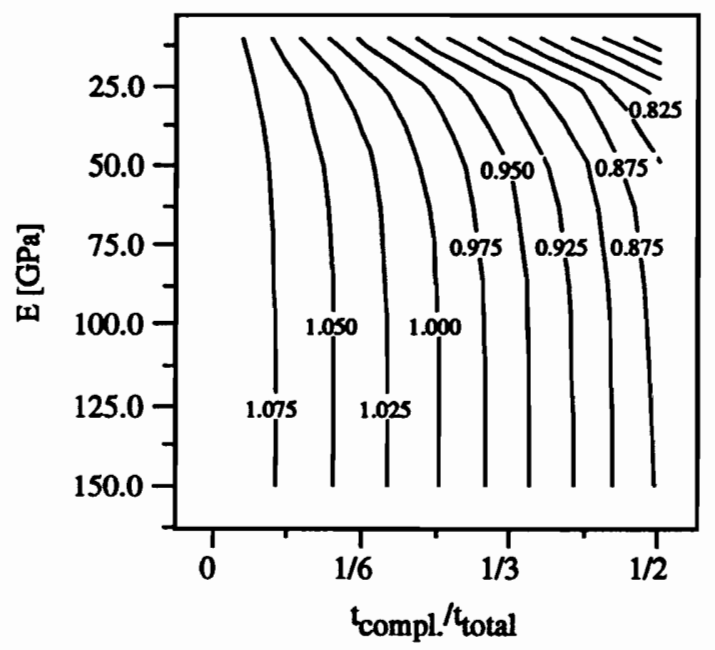

(a) $\bar{\sigma}_{1(\max )}$

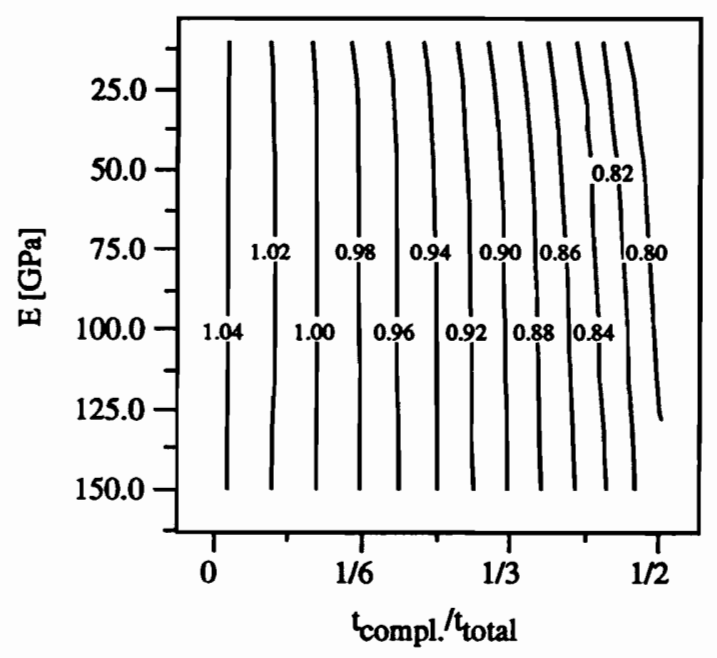

(c) $\bar{\sigma}_{z(\operatorname{mex})}$

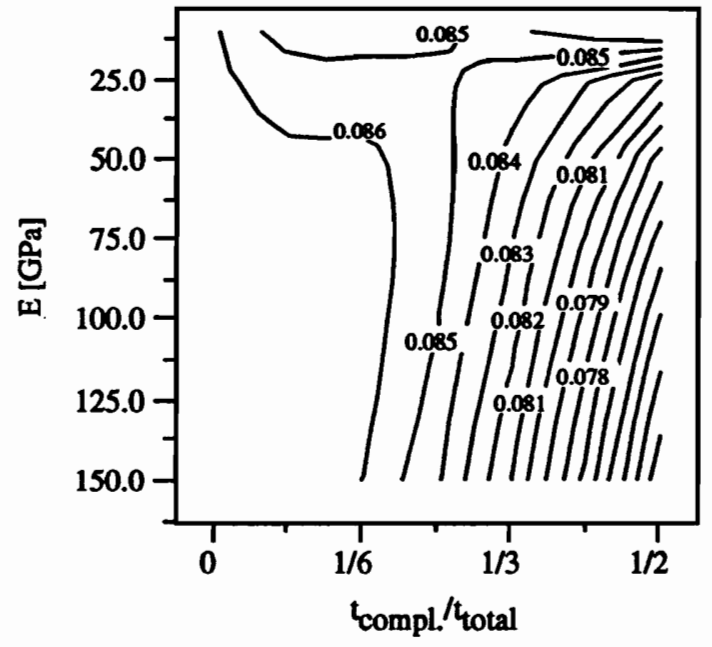

(b) $\bar{\sigma}_{2(\max )}$

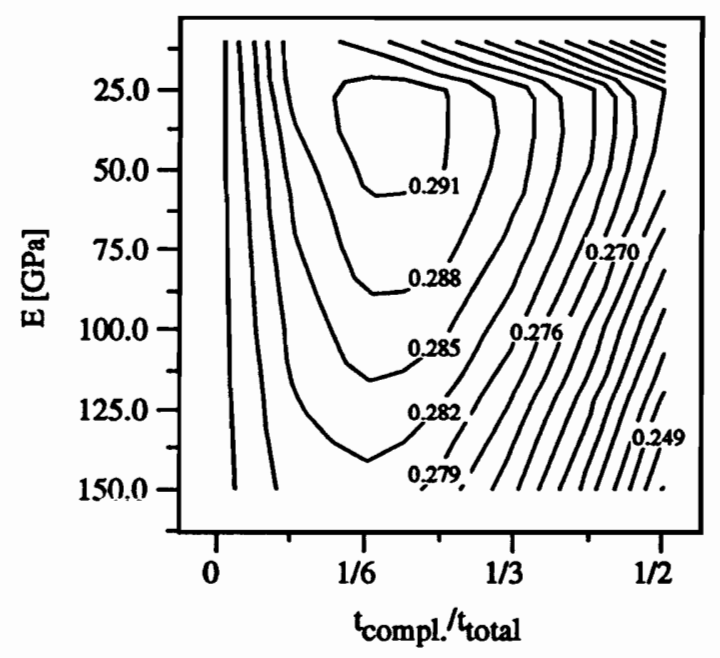

(d) $\bar{\tau}_{12(\max )}$

Figure 6.19: Effect of thickness and modulus of elasticity of the compliant coating on the maximum stresses in the $\mathrm{C} / \mathrm{C}$ substrate. 
the modulus and the coefficient of thermal expansion, the maximum stress components in the substrate and coatings were calculated. In Figs. 6.20 and 6.21, contour plots for the variations of the stress components with the two parameters are compared for the $\mathrm{SiC}$ coating and the compliant coating.

In Fig. 6.20a it can be seen that for coefficients of thermal expansion higher than the room temperature coefficient of thermal expansion of the $\mathrm{SiC}$ coating $\left(4.3 \times 10^{-6} /{ }^{\circ} \mathrm{C}\right)$, the maximum tangential stress $\bar{\sigma}_{s}$ in the $\mathrm{SiC}$ coating decreases slightly with increasing modulus of the compliant coating. The change of the maximum tangential stress in the $\mathrm{SiC}$ coating with increasing modulus in the compliant coating is small when the coefficient of thermal expansion is small. In contrast, the maximum tangential stress in the compliant coating (Fig. 6.20b) rapidly increases with increasing modulus and with increasing coefficient of thermal expansion of the compliant coating. For moduli larger than about $100 \mathrm{GPa}$, the maximum tangential stress in the compliant coating may reach values higher than in the $\mathrm{SiC}$ coating, despite the modulus being only $25 \%$ or less of the room temperature modulus of the SiC. Therefore, if the modulus of the compliant coating is increased, it is likely that failure will initiate in the compliant coating and not in the $\mathrm{SiC}$ coating. For coefficients of thermal expansion of the compliant coating less than the room temperature coefficient of thermal expansion of the $\mathrm{SiC}$, the maximum tangential stresses in the compliant coating are compressive. The magnitude of these maximum tangential stresses also increases rapidly with increasing modulus.

The maximum normal stresses $\bar{\sigma}_{n}$ in the $\mathrm{SiC}$ coating and the compliant coating are compared in Figs. 6.20c and d, respectively. The normal stress in the $\mathrm{SiC}$ coating is insensitive to changes in the modulus of the compliant coating when the coefficient of thermal expansion is close to its room temperature value, but is otherwise sensitive to modulus changes, and changes significantly with changing coefficient of thermal expansion. In fact, for high moduli and high coefficients of thermal expansion, the normal stress in the SiC coating becomes tensile and may cause delamination of the coatings. While the normal stress in the compliant coating is also somewhat insensitive to changes in the modulus for low coefficient 
of thermal expansion, it is insensitive to changes in the coefficient of thermal expansion for larger coefficients of thermal expansion. The overall range of the normal stress in the compliant coating in parameter space is much smaller for the compliant coating than it is for the SiC coating.

The contours for the axial stress in the SiC coating and in the compliant coating shown in figs 6.21a and $b$, respectively, are very similar to the contours of the maximum tangential stresses in these coatings. As was the case for the maximum tangential stress, the maximum axial stress in the compliant coating changes rapidly with increasing modulus and coefficient of thermal expansion, even changing sign with increasing coefficient of thermal expansion, so that the compliant coating may fail before the $\mathrm{SiC}$ coating for high moduli and high coefficients of thermal expansion.

The change of the maximum shear stress in the $\mathrm{SiC}$ coating and the compliant coating with the modulus and the coefficient of thermal expansion of the compliant coating is compared in figs 6.21c and d. As can be seen in Fig. 6.21c, the shear stress in the SiC coating has its lowest value for a high coefficient of thermal expansion and a low modulus of the compliant coating, but is generally insensitive to changes in those properties. The shear stress in the $\mathrm{SiC}$ coating increases from its lowest value if both the modulus and coefficient of thermal expansion increase or decrease. The shear stress in the compliant coating increases with both the coefficient of thermal expansion and the modulus.

It was shown in Section 6.4.2 that the maximum tangential stress in the $\mathrm{SiC}$ coating decreases for increasing coefficient of thermal expansion of the compliant coating (see Fig. 6.14a). In this section it was shown that the maximum tangential stress in the $\mathrm{SiC}$ coating decreases only slowly for increasing modulus of the compliant coating (see Fig. 6.17a). Further it was shown that the maximum tangential stress in the compliant coating increases rapidly with increasing modulus for larger coefficients of thermal expansion (see Fig. 6.20b). Therefore it appears that in order to obtain the lowest possible stress in the $\mathrm{SiC}$ coating without generating excessive stresses in the compliant coating, a high coefficient of thermal expansion and a low to moderate modulus of elasticity for the compliant coating should be 
used. For high coefficients of thermal expansion and moduli of elasticity of the compliant coating, the compliant coating might fail before the $\mathrm{SiC}$ coating.

\subsubsection{Influence of Orthotropic Compliant Coatings}

In Sections 6.4.2 and 6.4.3 the effect of the variation of the coefficient of thermal expansion and the modulus of elasticity of the compliant coating on the stresses in the coatings and the substrate was discussed. All the property variations in these sections were for isotropic coating materials, i.e., the compliant coating was isotropic for all property combinations. Since it may be possible to create orthotropic coatings in the deposition process, or orthotropic coatings may be an inherent result of that process, in this section the effect of an orthotropic compliant coating on the stresses in the coatings and the substrate is investigated. The properties varied were the coefficients of thermal expansion and the moduli of elasticity. Though the properties of a material generally do not vary independently in reality, the effects of varying the coefficients of thermal expansion and the moduli of elasticity were studied separately. This was done to better be able to evaluate the effects of each property independently. Therefore, first stresses for an orthotropic variation of the coefficients of thermal expansion, and then stresses for an orthotropic variation of the moduli of elasticity, of the compliant coatings were studied. It should be noted here that the principal material directions for the orthotropic coatings are aligned with the $s-n-z-$ coordinate system of Fig. 5.1 on page 59.

\subsubsection{Orthotropic Variation of the Coefficient of Thermal Expansion}

Two separate cases were analyzed for the orthotropic variation of the coefficient of thermal expansion of the compliant coating. These were a transverse and then an inplane variation of the coefficient of thermal expansion, while all other properties were kept constant. For the transverse variation of the coefficient of thermal expansion, the coefficient of thermal expansion in the thickness or $n$-direction, as indicated in Fig. 5.1, was varied from $\alpha_{n}=$ 0 to $25 \times 10^{-6} /{ }^{\circ} \mathrm{C}$. The inplane coefficients of thermal expansion $\alpha_{s}$ and $\alpha_{z}$ were kept at 


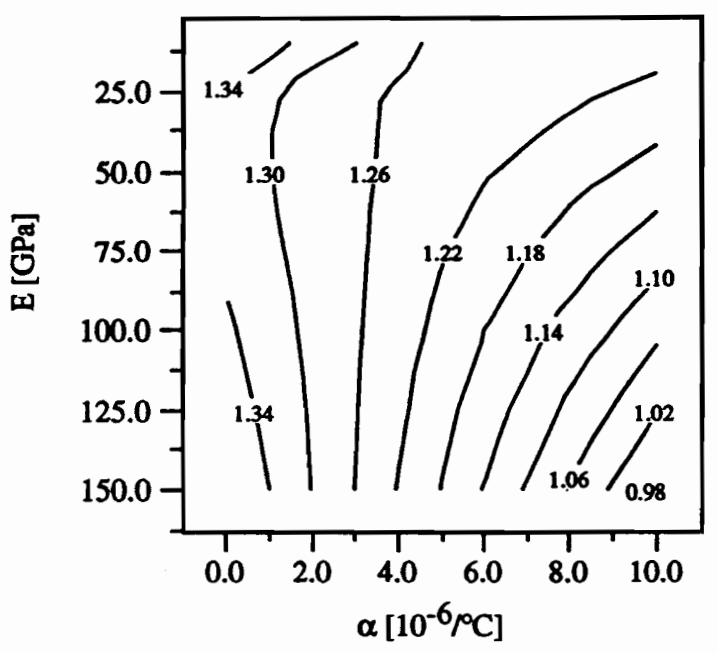

(a) $\bar{\sigma}_{s, \operatorname{mex}(S i C)}$

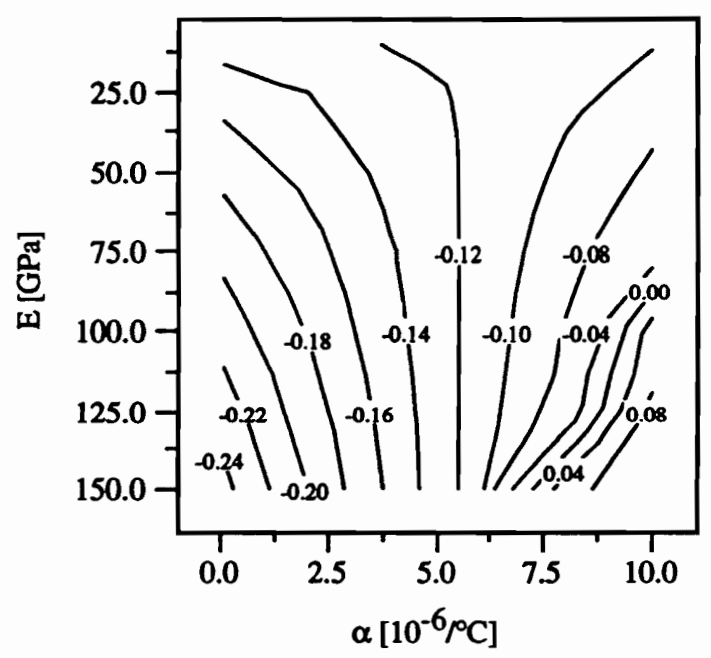

(c) $\bar{\sigma}_{n, \max (S i C)}$

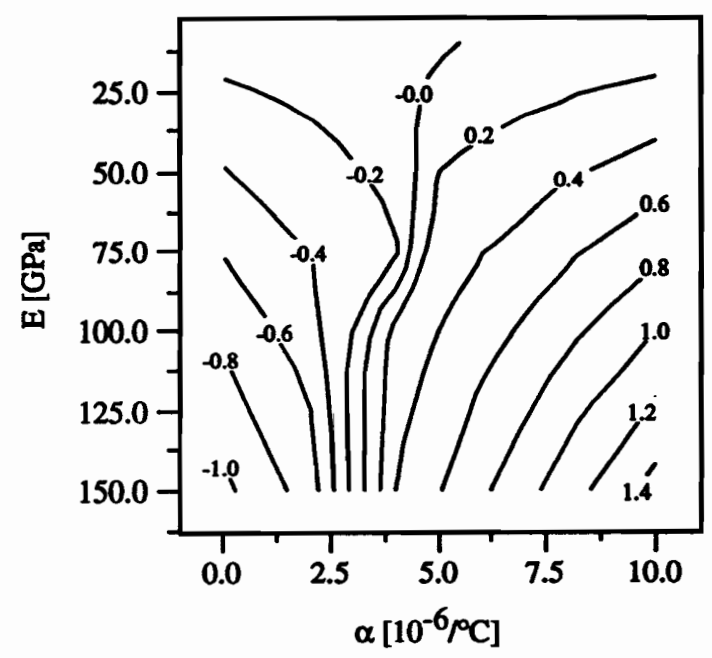

(b) $\bar{\sigma}_{s, \max (\text { compl.) }}$

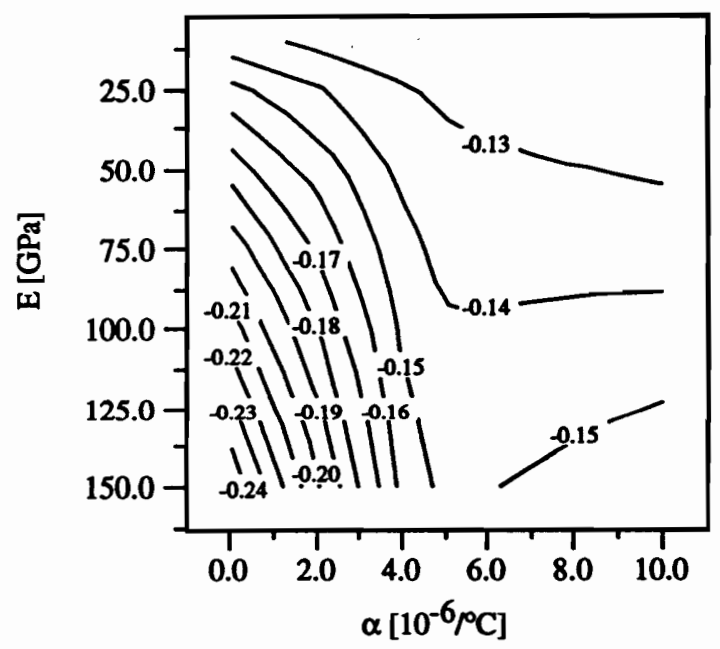

(d) $\bar{\sigma}_{n, \max (\text { compl.) }}$

Figure 6.20: Effect of coefficient of thermal expansion and modulus of the compliant coating on stresses in the $\mathrm{SiC}$ coating and the compliant coating. Comparison of maximum tangential stresses and maximum normal stresses. 


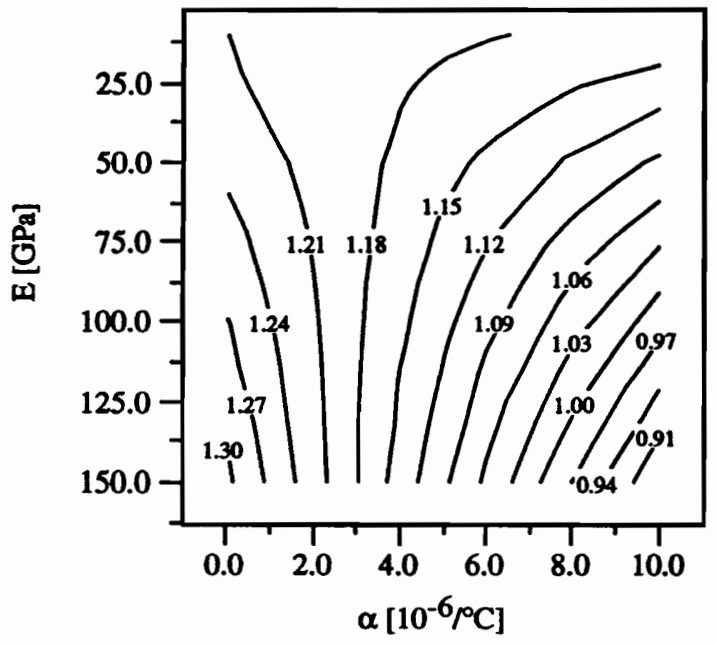

(a) $\bar{\sigma}_{\boldsymbol{x}, \operatorname{mex}(\mathrm{SiC})}$

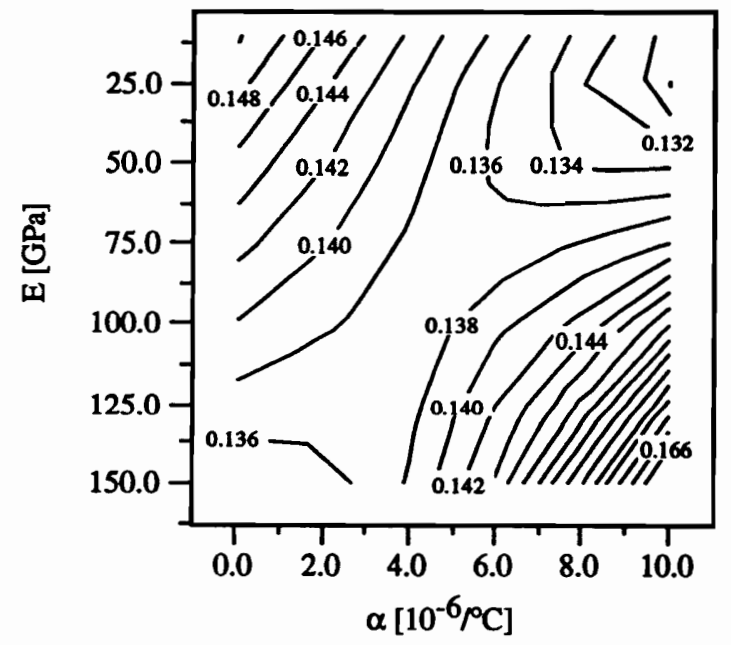

(c) $\bar{\tau}_{a n, \max (S i C)}$

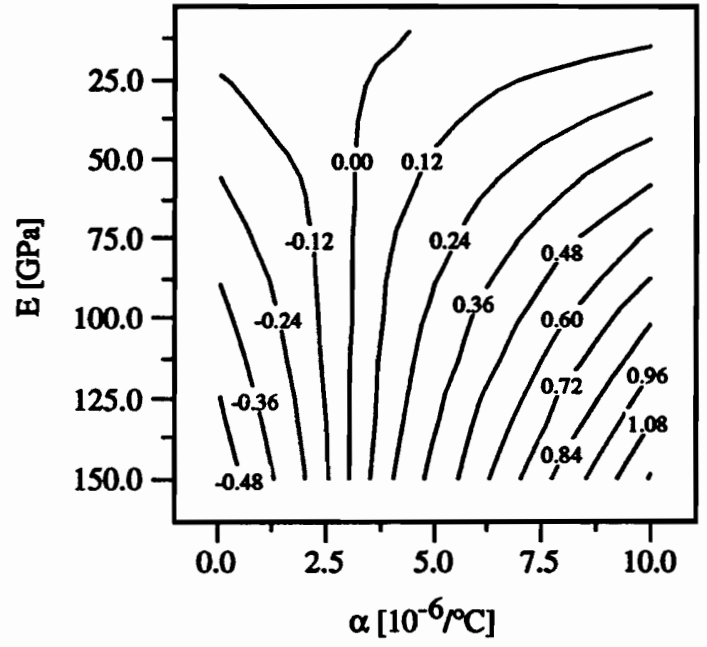

(b) $\bar{\sigma}_{x, \max (\text { compl.) }}$

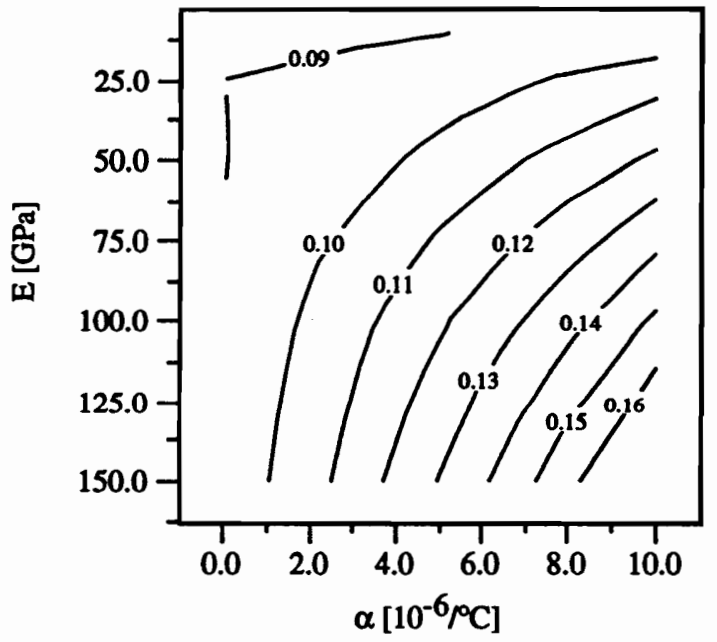

(d) $\bar{\tau}_{e n, \max (\text { compl.) }}$

Figure 6.21: Effect of coefficient of thermal expansion and modulus of the compliant coating on stresses in the $\mathrm{SiC}$ coating and the compliant coating. Comparison of maximum axial stresses and maximum shear stresses. 
their baseline values $\alpha_{s}=\alpha_{z}=3 \times 10^{-6} /{ }^{\circ} \mathrm{C}$. For the inplane variation of the coefficients of thermal expansion, $\alpha_{s}$ and $\alpha_{z}$ were varied between 0 and $25 \times 10^{-6} /{ }^{\circ} C$, while the transverse coefficient of thermal expansion $\alpha_{n}$ was kept at its baseline value. Because, as was discussed before, the thickness of the compliant coating may significantly influence the stresses in the coatings and the substrate, the results are shown as contour plots of the maximum stresses as a function of the coefficients of thermal expansion and the thickness of the compliant coating. The contour plots of the maximum stresses as a function of the thickness and the transverse coefficient of thermal expansion of the compliant coating are shown in Figs. 6.22 - 6.24 for the $\mathrm{SiC}$ coating, the compliant coating, and the substrate, respectively. The contour plots of the maximum stresses as a function of the thickness and the inplane coefficient of thermal expansion of the compliant coating are shown in Figs. 6.25 - 6.27 for the $\mathrm{SiC}$ coating, the compliant coating, and the substrate, respectively. As can be seen in Fig. 6.22a for the variation of the transverse coefficient of thermal expansion and in Fig. 6.25a for the variation of the inplane coefficient of thermal expansion of the compliant coating, the lowest obtained maximum tangential stresses in the $\mathrm{SiC}$ coating are $\bar{\sigma}_{s(\mathrm{SiC})}=1.18$ and $\bar{\sigma}_{s(\mathrm{SiC})}=1.20$, respectively. Both of these are larger than the lowest maximum tangential stress for the isotropic variation of the coefficient of thermal expansion, which was $\bar{\sigma}_{s(\mathrm{SiC})}=1.10$ (see Fig. 6.14a).

For both the variations of the transverse and the inplane coefficients of thermal expansion, the contours for the tangential stress in the $\mathrm{SiC}$ coating in Figs. 6.22a and 6.25a, respectively, are very similar to the contours obtained for the isotropic variation of the coefficient of thermal expansion (Fig. 6.14a). As was the case for the isotropic variation of the coefficient of thermal expansion(Fig. 6.14a), the maximum tangential stress $\bar{\sigma}_{s}$ in the SiC coating decreases for increasing compliant coating thickness for given coefficients of thermal expansion until $\bar{\sigma}_{s}$ reaches a minimum and then increases again. The lowest maximum stress $\bar{\sigma}_{s}$ is obtained for high inplane coefficients of thermal expansion and thickness ratios of about $t_{\text {compl. }} / t_{\text {total }}=1 / 6$ for the variation of the transverse coefficient of thermal expansion and $t_{\text {compl. }} / t_{\text {total }}=1 / 4$ for the variation of the inplane coefficient of thermal expansion. 
The contour plots for the axial stress $\bar{\sigma}_{z}$ in the $\mathrm{SiC}$ coating are also similar for the isotropic, the transverse, and the inplane variations of the coefficients of thermal expansion of the compliant coating (Figs. 6.14c, 6.22c, and 6.25c). For all cases the axial stress increases with increasing compliant coating thickness and is insensitive to changes in the coefficient of thermal expansion of the compliant coating. As for an isotropic variation of the coefficient of thermal expansion, the normal stress $\bar{\sigma}_{n}$ in the $\mathrm{SiC}$ coating decreases with increasing compliant coating coefficient of thermal expansion and thickness (Fig. 6.14b) for both the transverse and inplane variations of the coefficients of thermal expansion (Figs. 6.22b and $6.25 \mathrm{~b})$. For the inplane variation of the coefficient of thermal expansion, the shear stress $\bar{\tau}_{s n}$ in the SiC coating shown in Fig. 6.25d is somewhat insensitive to the coefficient of thermal expansion, and decreases with increasing thickness. Again the isotropic and transverse variations are very similar. In fact, the isotropic variation (Fig. 6.14a), the transverse variation (Fig. 6.22a), and the inplane variation (Fig. 6.25a) all look very similar for all stress components.

The contours for all stress components in the compliant coating for the isotropic variation of the coefficient of thermal expansion (Fig. 6.15) and the inplane variation of the coefficient of thermal expansion (Fig. 6.26) are very similar. The same applies to the contours for the normal and shear stresses for the variation of the transverse coefficient of thermal expansion only (Figs. $6.23 \mathrm{~b}$ and d). The tangential stress $\bar{\sigma}_{s}$ and the axial stress $\bar{\sigma}_{z}$ in the compliant coating for both the isotropic variation case and the inplane variation case are insensitive to changes in the thickness and increase significantly with increasing coefficients of thermal expansion. For a change of the transverse coefficient of thermal expansion only, the properties in the tangential and axial directions do not change and, therefore, the magnitudes of the variations in the tangential and axial stresses (Figs. 6.23a and c) are much smaller compared to the cases in Figs. $6.15 \mathrm{a}$ and $\mathrm{c}$, and $6.26 \mathrm{a}$ and $\mathrm{c}$.

The principal material direction stresses $\bar{\sigma}_{1}$ and $\bar{\sigma}_{z}$ in the substrate are insensitive to the variation of the coefficient of thermal expansion in the compliant coating for all cases (Figs. 6.16a and c, 6.24a and c, and 6.27a and c). Because of the low modulus $E_{2}$ of the 
substrate, the principal material direction stress in the 2-direction is small for all cases. The variations of this stress with the coefficient of thermal expansion and the thickness are not significant for any case. For all cases of the variation in the coefficient of thermal expansion, the shear stress in the substrate increases similarly with increasing coefficient of thermal expansion for intermediate thicknesses of the compliant coating, and is insensitive to changes in the coefficient of thermal expansion for small thicknesses.

In summary, variations of the transverse and inplane coefficients of thermal expansion have a similar effect on the stresses in the $\mathrm{SiC}$ coating and the substrate, and reduce the tangential stress in the $\mathrm{SiC}$ coating. However, the lowest tangential stress in the $\mathrm{SiC}$ coating was obtained for an isotropic increase of the coefficient of thermal expansion. For the transverse variation of the coefficient of thermal expansion, the decrease in the tangential stress in the $\mathrm{SiC}$ coating was achieved without increasing the tangential stress in the compliant coating.

\subsubsection{Orthotropic Variation of the Modulus of Elasticity}

To further study the effect of an orthotropic coating on the stresses in the coatings and the substrate, parametric studies for an orthotropic variation of the modulus of elasticity were conducted. The cases considered were:

- A quasi-isotropic coating, for which the moduli of elasticity $E_{s}, E_{n}$, and $E_{z}$ of the compliant coating were increased equally in all material directions, but the Poisson's ratios, the shear moduli, and the coefficients of thermal expansion were kept at their baseline values. This is in contrast to the discussion in Section 6.4.3, where the shear moduli were allowed to vary in proportion to the moduli of elasticity through the classic relation $G=E /(2(1+\nu))$.

- An orthotropic material, for which the transverse modulus of elasticity $E_{n}$ of the compliant coating was increased independent of all other properties. 


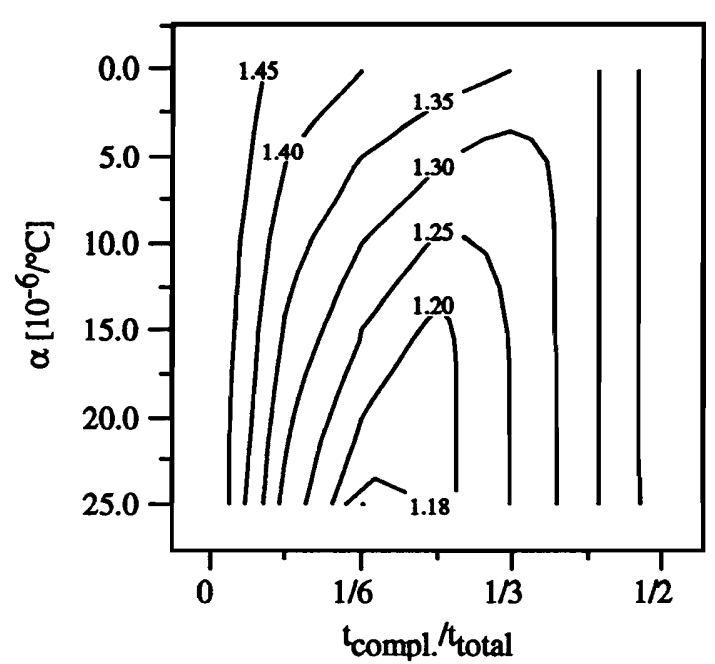

(a) $\bar{\sigma}$,

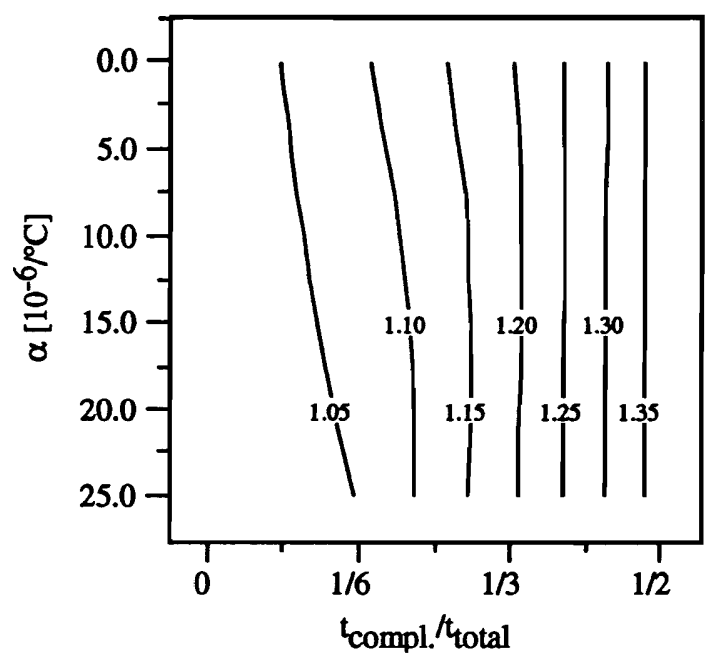

(c) $\bar{\sigma}_{z}$

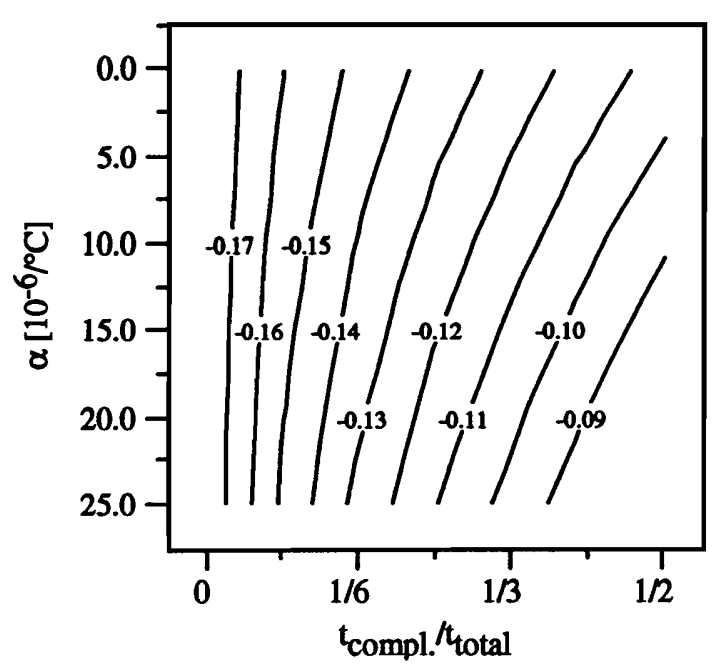

(b) $\bar{\sigma}_{n}$

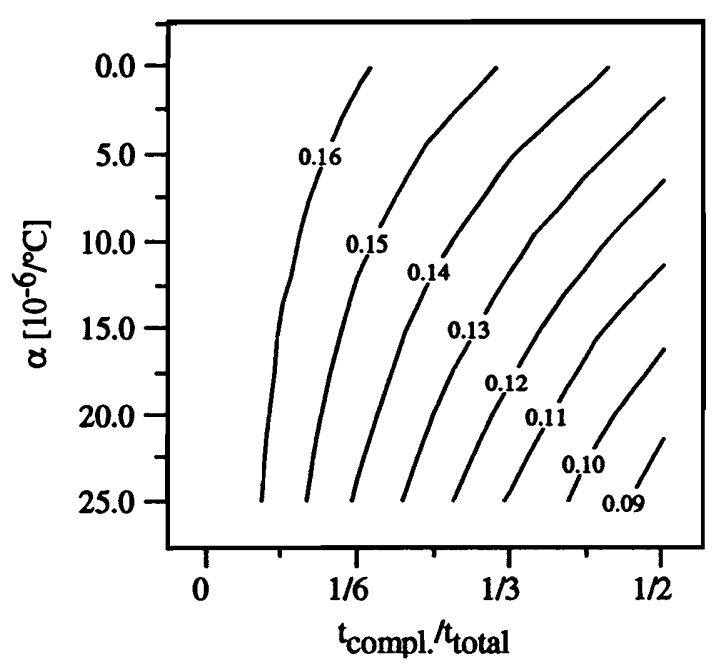

(d) $\bar{\tau}_{s n}$

Figure 6.22: Effect of thickness and transverse coefficient of thermal expansion of the compliant coating on the maximum stresses in the $\mathrm{SiC}$ coating. 


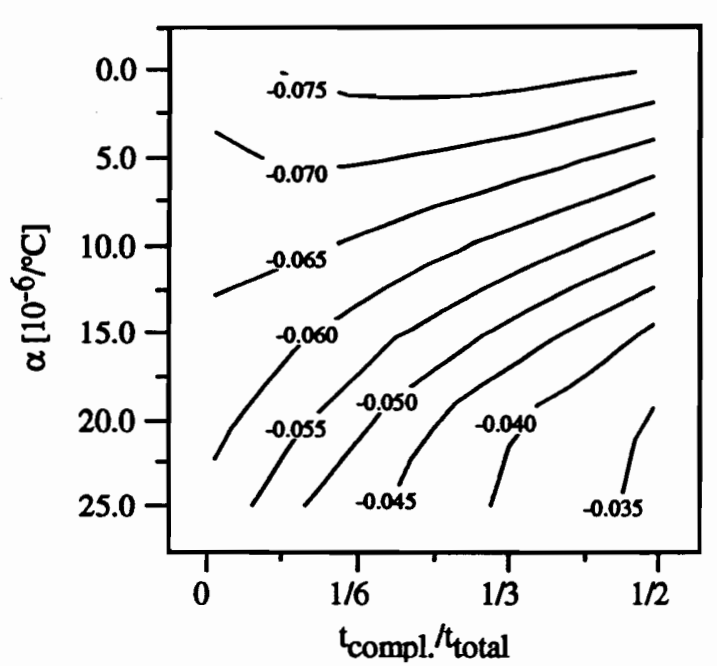

(a) $\bar{\sigma}$.

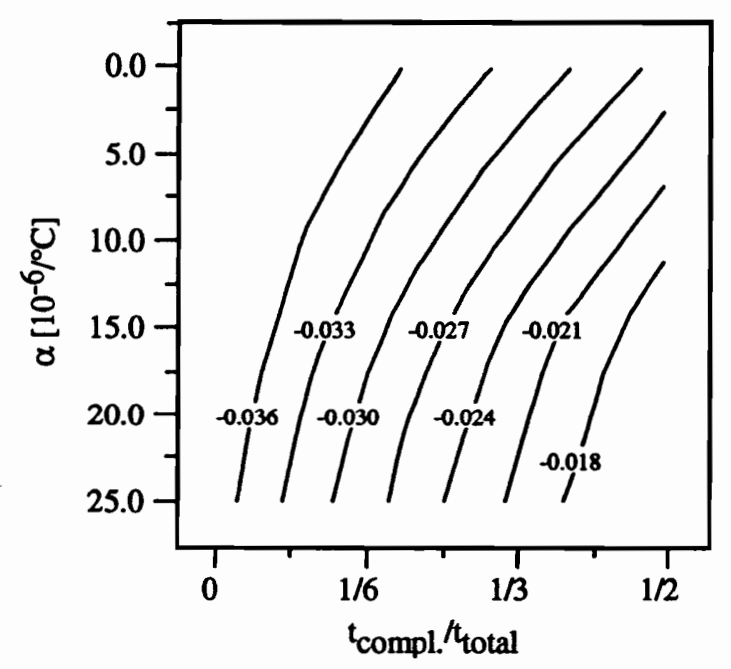

(c) $\bar{\sigma}_{z}$

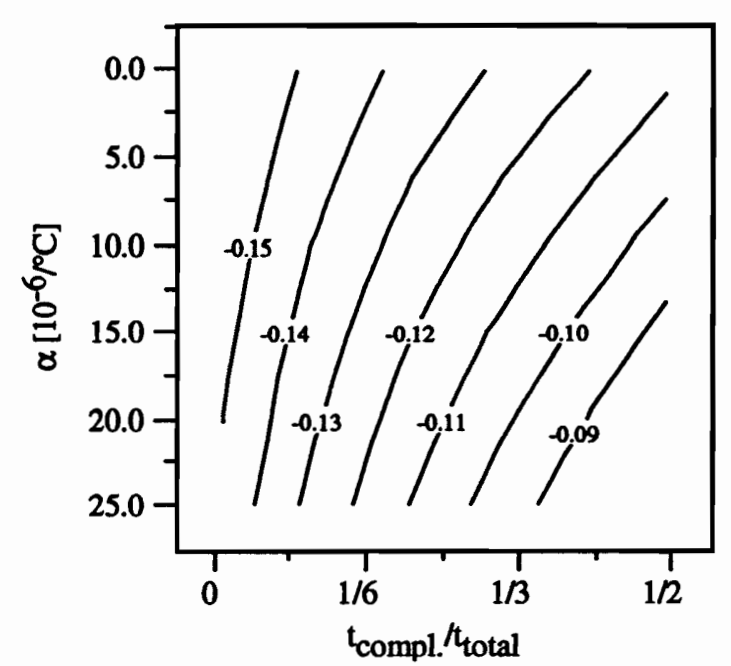

(b) $\bar{\sigma}_{n}$

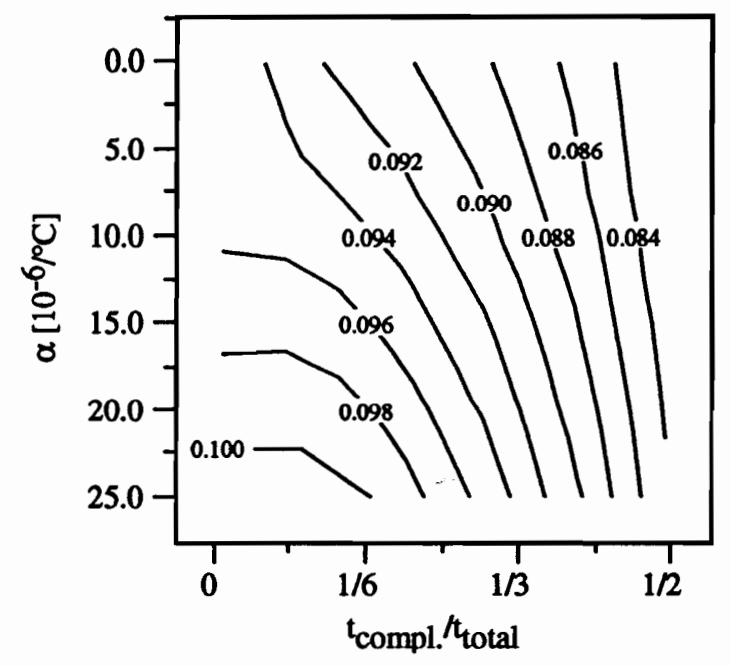

(d) $\bar{\tau}_{a n}$

Figure 6.23: Effect of thickness and transverse coefficient of thermal expansion of the compliant coating on the maximum stresses in the compliant coating. 


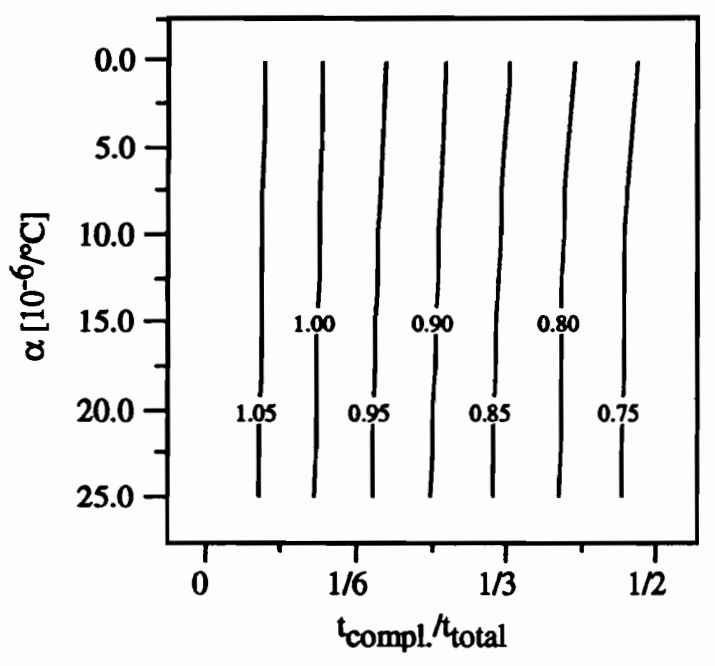

(a) $\bar{\sigma}_{1}$

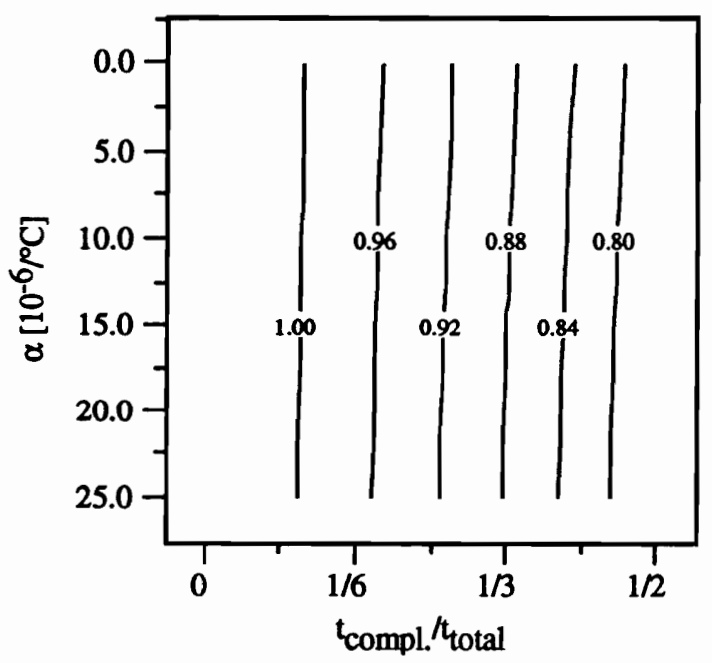

(c) $\bar{\sigma}_{\boldsymbol{z}}$

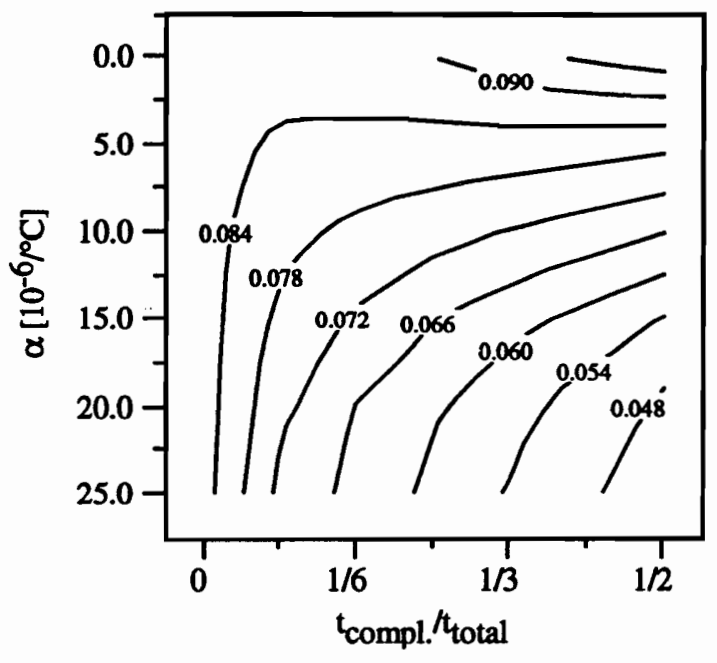

(b) $\bar{\sigma}_{2}$

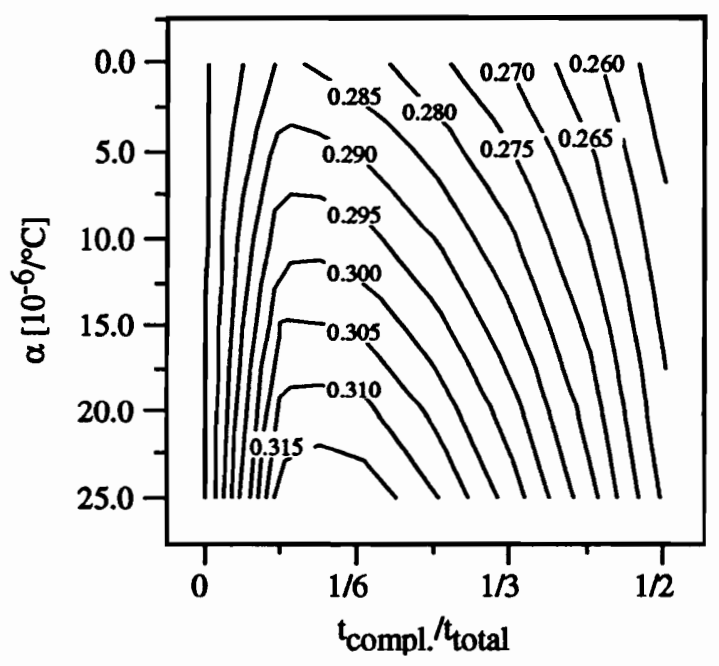

(d) $\bar{\tau}_{12}$

Figure 6.24: Effect of thickness and transverse coefficient of thermal expansion of the compliant coating on the maximum stresses in the $\mathrm{C} / \mathrm{C}$ substrate. 


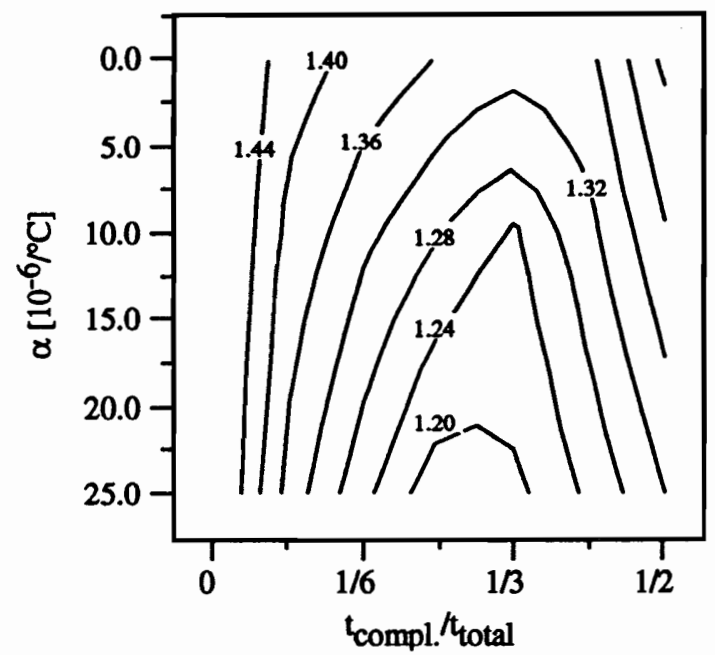

(a) $\bar{\sigma}_{\boldsymbol{s}}$

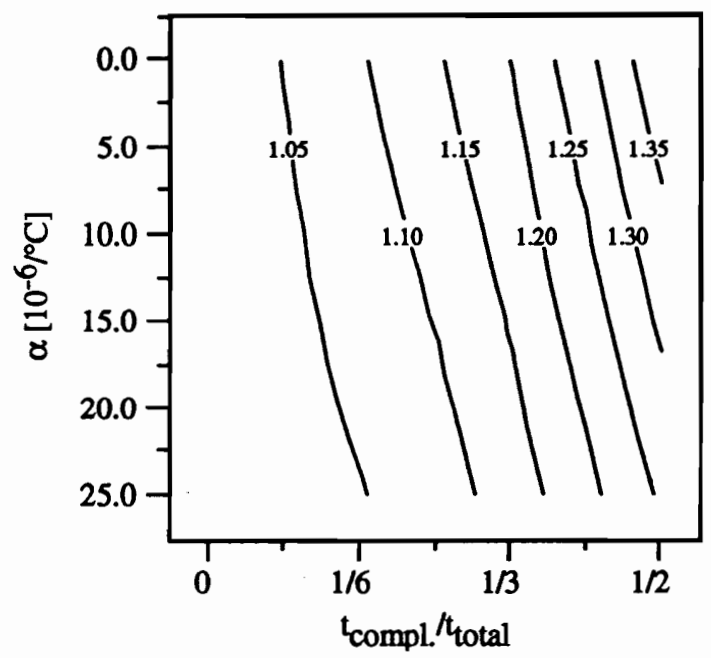

(c) $\bar{\sigma}_{z}$

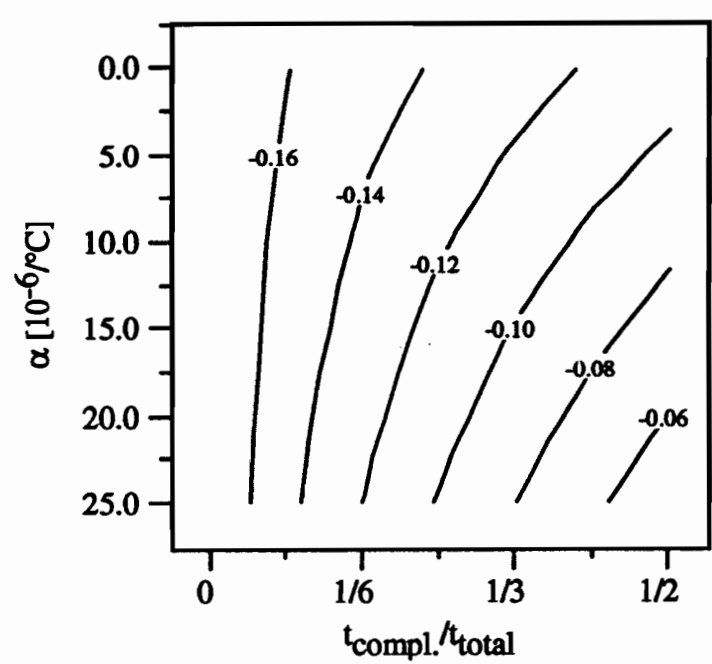

(b) $\bar{\sigma}_{n}$

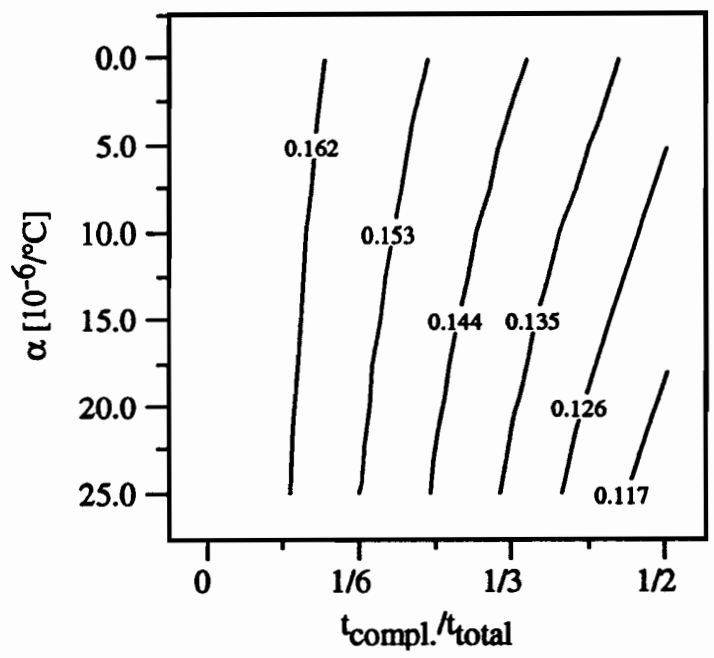

(d) $\bar{\tau}_{e n}$

Figure 6.25: Effect of thickness and inplane coefficients of thermal expansion of the compliant coating on the maximum stresses in the $\mathrm{SiC}$ coating. 


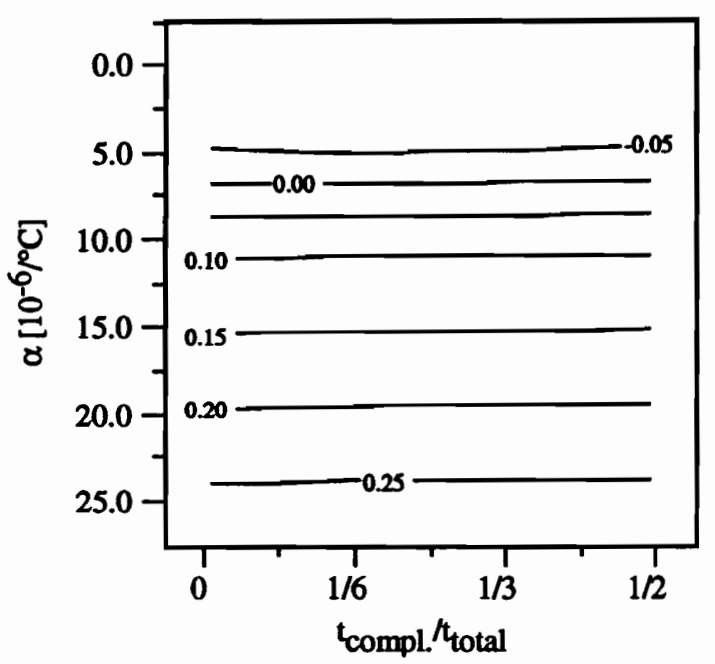

(a) $\bar{\sigma}$.

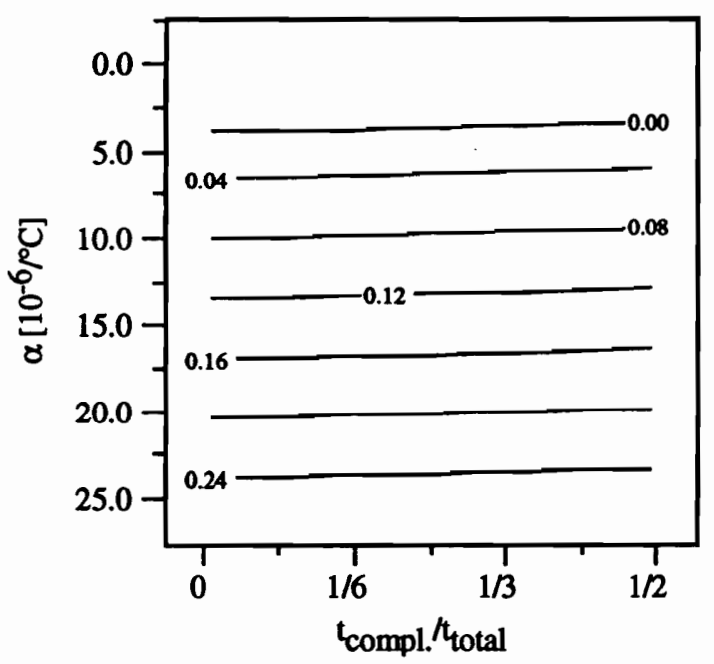

(c) $\bar{\sigma}_{x}$

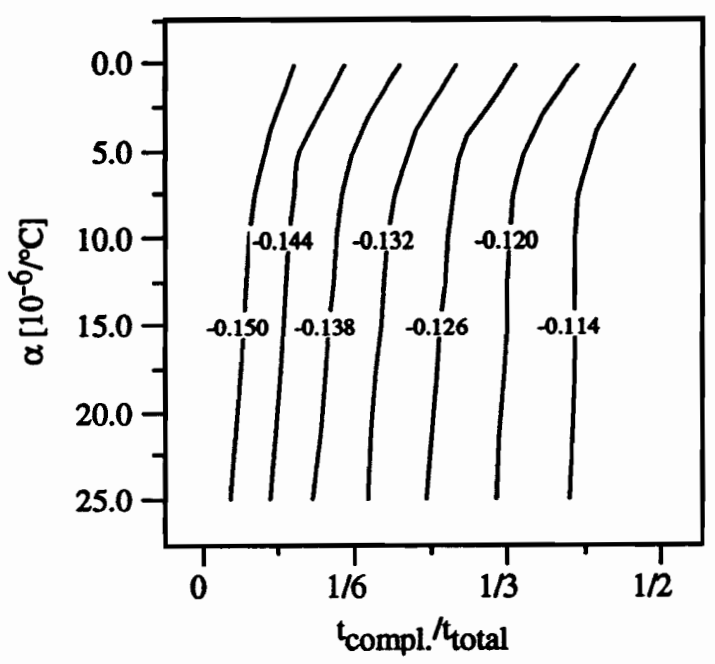

(b) $\bar{\sigma}_{n}$

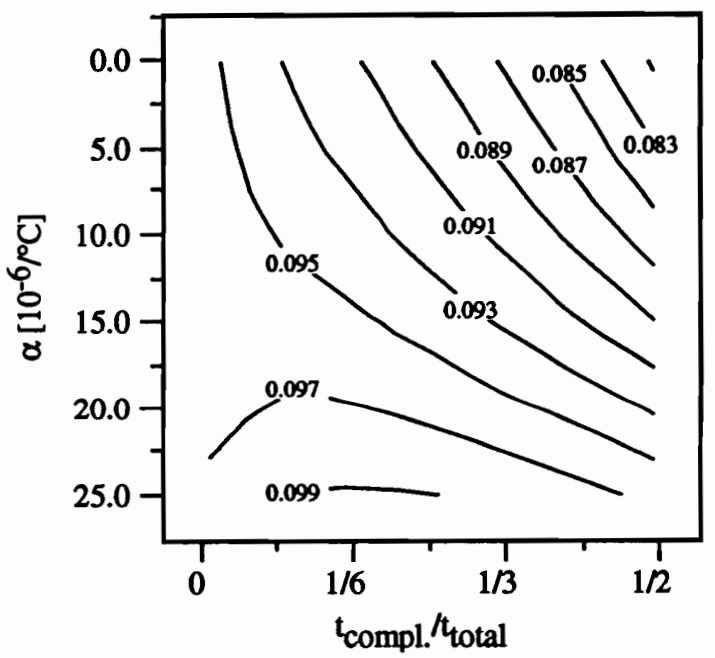

(d) $\bar{\tau}_{e n}$

Figure 6.26: Effect of thickness and inplane coefficients of thermal expansion of the compliant coating on the maximum stresses in the compliant coating. 


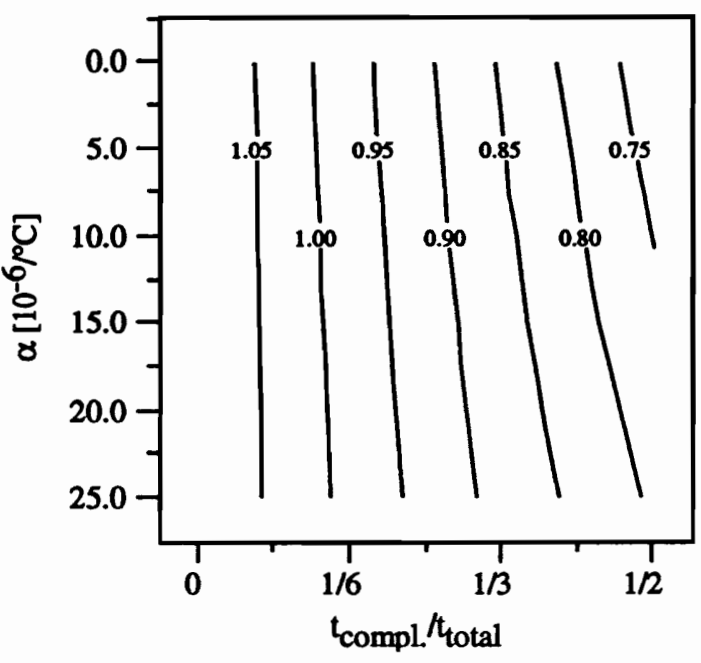

(a) $\bar{\sigma}_{1}$

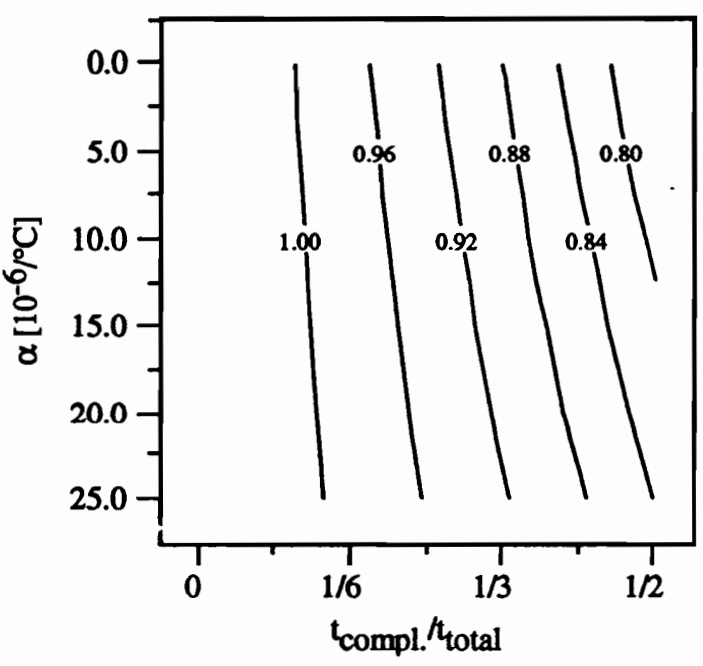

(c) $\bar{\sigma}_{\boldsymbol{z}}$

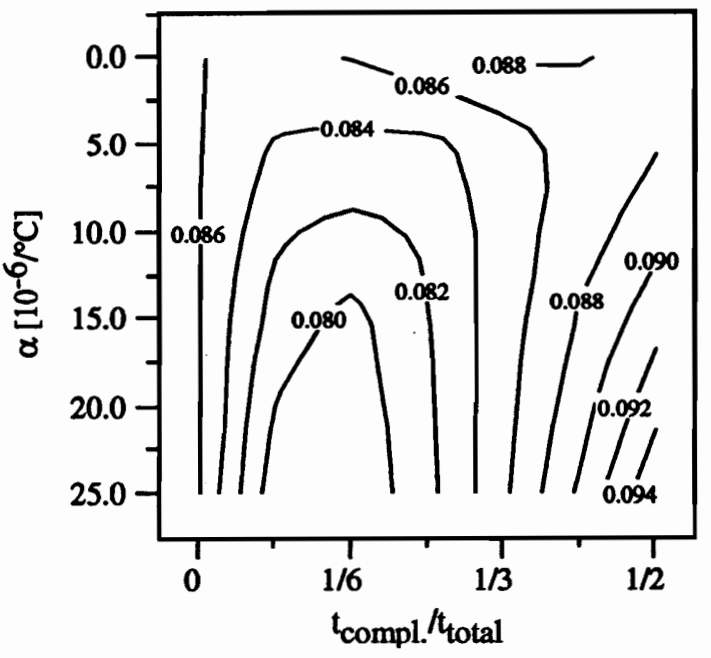

(b) $\bar{\sigma}_{2}$

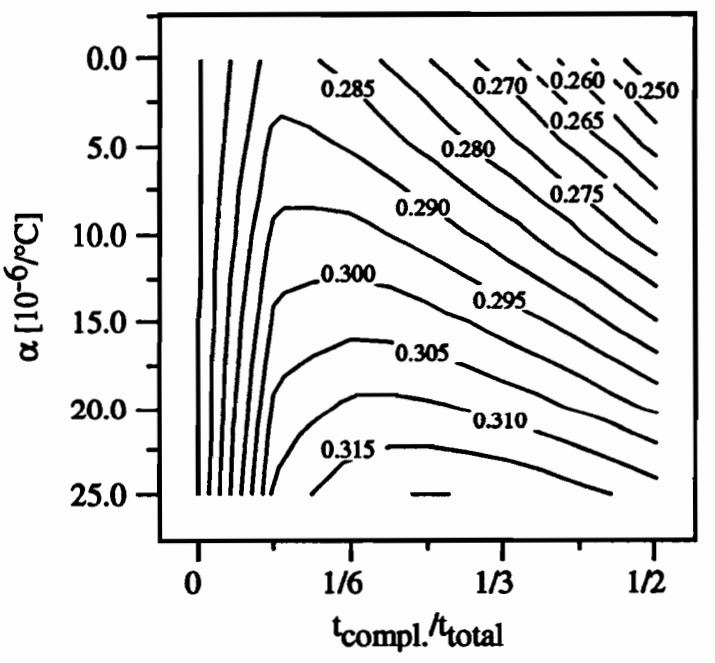

(d) $\bar{\tau}_{12}$

Figure 6.27: Effect thickness and inplane coefficients of thermal expansion of the compliant coating on the maximum stresses in the $\mathrm{C} / \mathrm{C}$ substrate. 
- An orthotropic material, for which the inplane moduli of elasticity $E_{s}$ and $E_{z}$ of the compliant coating were increased independent of all other properties.

For all cases the respective moduli of elasticity were varied between $10 \mathrm{GPa}$ and $150 \mathrm{GPa}$. Maximum stresses in the coatings and the substrate were calculated as a functions of both the modulus of elasticity and the thickness of the compliant coating. In Figs. $6.28-6.36$ the contour plots of the stresses for the $\mathrm{SiC}$ coating, the compliant coating, and the substrate for the three cases are shown.

In the $\mathrm{SiC}$ coating, the tangential stress $\bar{\sigma}_{s}$ changes similarly for the case of the inplane variation of the moduli of elasticity (Fig. 6.34a) and the isotropic variation of the moduli of elasticity (Fig. 6.17a). For both cases the stress is insensitive to modulus changes for small and large thicknesses of the compliant coating. For intermediate thicknesses, the tangential stress decreases with increasing modulus. In contrast, for the quasi-isotropic and the transverse variations of the moduli (Figs. 6.28a and 6.31a), the tangential stress increases with increasing moduli for intermediate thicknesses of the compliant coating. For these cases, the tangential stress is lowest for small moduli at about $t_{\text {compl. }} / t_{\text {total }}=1 / 3$. However, the lowest tangential stress for both the isotropic and the inplane variation of the moduli is smaller than it is for the quasi-isotropic and the transverse variation cases. The change of the normal stress $\bar{\sigma}_{n}$ for the cases of the isotropic and the quasi-isotropic variation of the moduli of elasticity of the compliant coating are similar (Figs. 6.17b and 6.28b). The same applies to the transverse and inplane variation cases (Figs. 6.31b and 6.34b). For all cases, the normal stress is compressive for all combinations of thickness and moduli of the compliant coating. The normal stress has its smallest magnitude for small moduli and large thickness, and it has its largest magnitude for large moduli and small thickness of the compliant coating. For the transverse and inplane variation cases, the normal stress is less sensitive to changes in the moduli of elasticity than it is for the other two cases. For all cases, the changes of the axial stress $\bar{\sigma}_{z}$ in the $\mathrm{SiC}$ coating with thickness and moduli of elasticity of the compliant coating are similar. The axial stress is insensitive to changes in the moduli and increases with increasing thickness. The change of the shear stress $\bar{\tau}_{s n}$ 
with thickness and moduli of the compliant coating is also similar for all cases. The shear stress is insensitive to changes in modulus and decreases with increasing thickness of the compliant coating.

In the compliant coating, the tangential stress $\bar{\sigma}_{s}$ is somewhat insensitive to changes of the thickness and increases with increasing moduli for the isotropic, the quasi-isotropic, and the inplane variations of the moduli of the compliant coating (Figs. 6.18a, 6.29a, and 6.35a, respectively). The increase in the tangential stress with increasing moduli is largest for the quasi-isotropic case. For the transverse variation of the modulus of the compliant coating, the overall changes of the tangential stress are insignificant compared to the other cases. This is the case because for the transverse variation of the modulus the tangential modulus remains at its low baseline value, and hence lower transverse stresses are generated. For all cases, the normal stress $\bar{\sigma}_{n}$ in the compliant coating has its lowest magnitude for low moduli and large thicknesses, and has its largest value for large moduli and low thicknesses of the compliant coating. As in the SiC coating, in the compliant coating the normal stress is compressive for all parameter combinations. The maximum axial stress $\bar{\sigma}_{z}$ in the compliant coating, for the quasi-isotropic and the inplane variation of the modulus, changes in the same way with the modulus and the thickness as in the isotropic case, though the magnitudes of the changes are somewhat different. For the transverse variation of the modulus the changes in this stress are insignificant. As with the tangential stress, this is due to the fact that for the transverse variation of the modulus, the modulus in the axial direction remains at its small baseline value. For the transverse and inplane variations of the moduli, the shear stress $\bar{\tau}_{s n}$ in the compliant coating is insensitive to changes in moduli and decreases with increasing thickness of the compliant coating. For the quasi-isotropic case the shear stress is also insensitive to changes in the moduli and decreases with increasing compliant coating thickness.

In the substrate, the maximum principal material direction stresses $\bar{\sigma}_{1}$ and $\bar{\sigma}_{z}$ vary similarly for the quasi-isotropic, transverse, and inplane variations of the moduli, Figs. 6.30a, $6.33 \mathrm{a}$, and 6.36a, respectively. The stresses are in the same range as for the isotropic case 
(Fig. 6.19a). For all orthotropic cases $\bar{\sigma}_{1}$ and $\bar{\sigma}_{z}$ are insensitive to changes in the moduli and decrease with increasing thickness of the compliant coating. The contours for the principal material direction stress $\bar{\sigma}_{2}$ in the substrate are also similar for the quasi-isotropic, transverse, and inplane cases. For all cases, this stress increases with increasing thickness and modulus of the compliant coating. The shear stress $\bar{\tau}_{s n}$ varies over the same range of magnitudes for all cases. It is largest for small thicknesses and smallest for large thicknesses of the compliant coating.

\subsubsection{Summary of Material Effects on Stresses in Coatings and Substrate}

In Sections 6.4.2 - 6.4.4, the effects of different material property variations of the compliant coating on the stresses in the coatings and substrate were investigated for different thicknesses of the compliant coating. Because the maximum tangential stress $\bar{\sigma}_{s}$ in the $\mathrm{SiC}$ coating is the largest stress and most likely the stress that would cause failure of the coatings, the results are summarized with emphasis on this stress component. The objective of the parametric material property studies was to perhaps find a combination of material properties and thickness of the compliant coating that would reduce the stresses in the $\mathrm{SiC}$ coating without having detrimental effects on the compliant coating and the substrate. The following conclusions can be drawn from the studies:

- An isotropic increase of the coefficient of thermal expansion of the compliant coating for a low modulus of the compliant coating resulted in a significantly reduced maximum tangential stress in the $\mathrm{SiC}$ coating compared to the baseline case (Fig. 6.14a). For low moduli of the compliant coating, no adverse effects on other stress components for changing the coefficient of thermal expansion were observed.

- An isotropic increase of the moduli of the compliant coating resulted in reduced maximum tangential stresses in the $\mathrm{SiC}$ coating for larger values of the coefficient of thermal expansion of the compliant coating (Fig. 6.20a). However, stresses in the compliant 


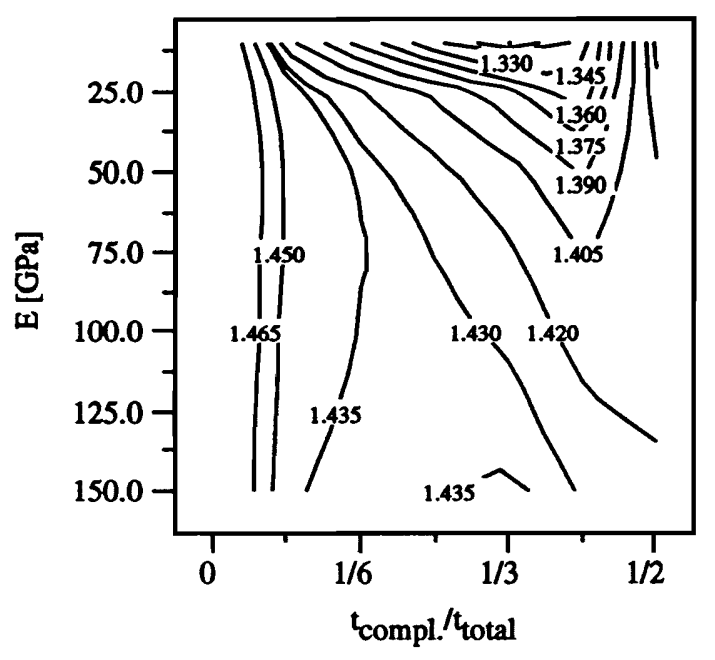

(a) $\bar{\sigma}_{a(\max )}$

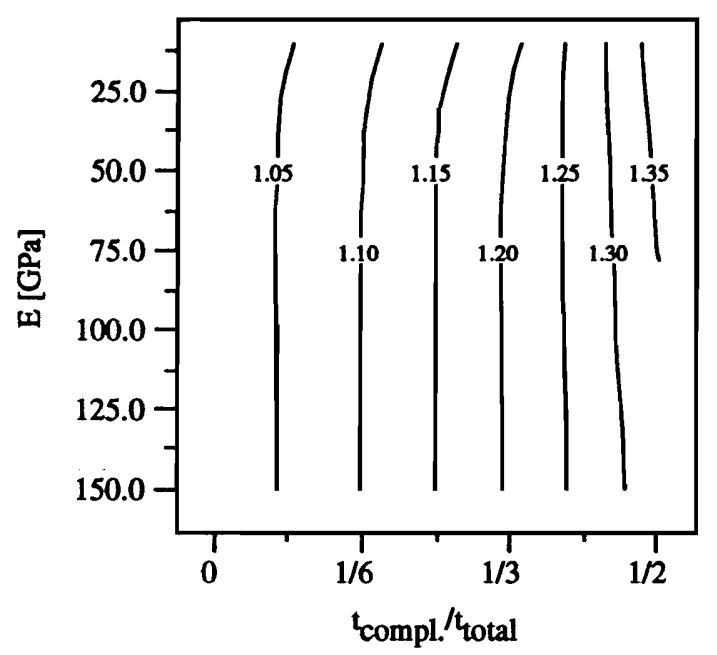

(c) $\bar{\sigma}_{z(\max )}$

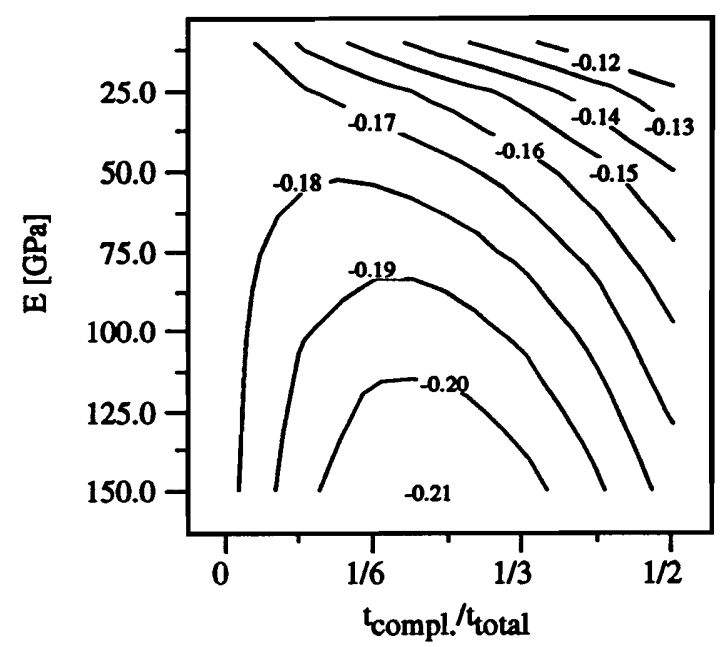

(b) $\bar{\sigma}_{n(\max )}$

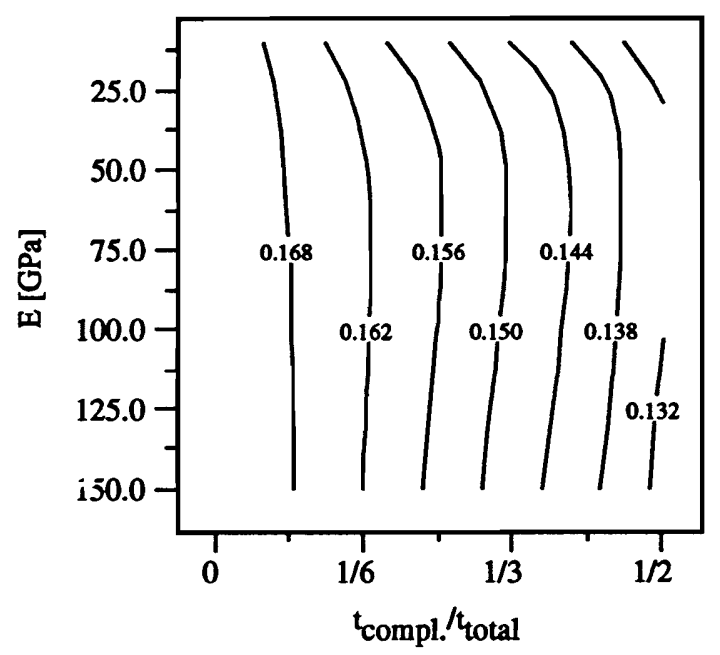

(d) $\bar{\tau}_{\ln (\max )}$

Figure 6.28: Effect of thickness and quasi-isotropic moduli of the compliant coating on the maximum stresses in the $\mathrm{SiC}$ coating. 


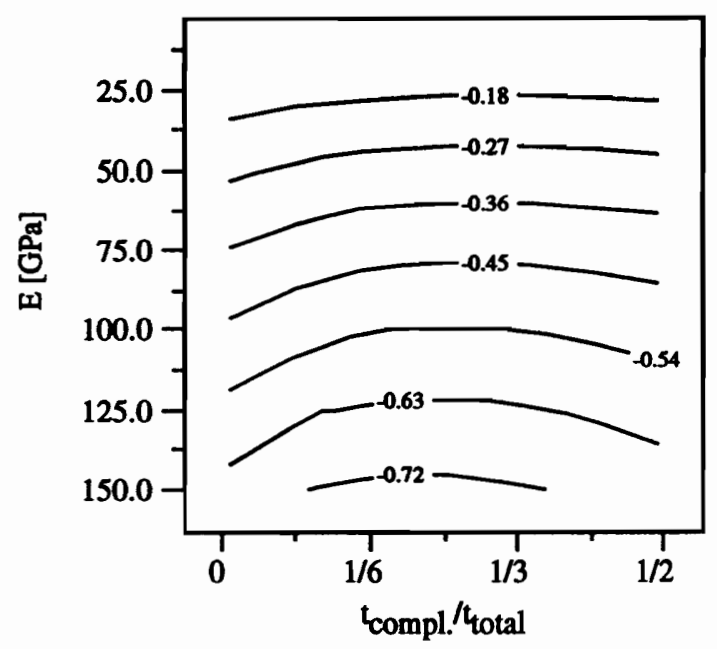

(a) $\bar{\sigma}_{s(\max )}$

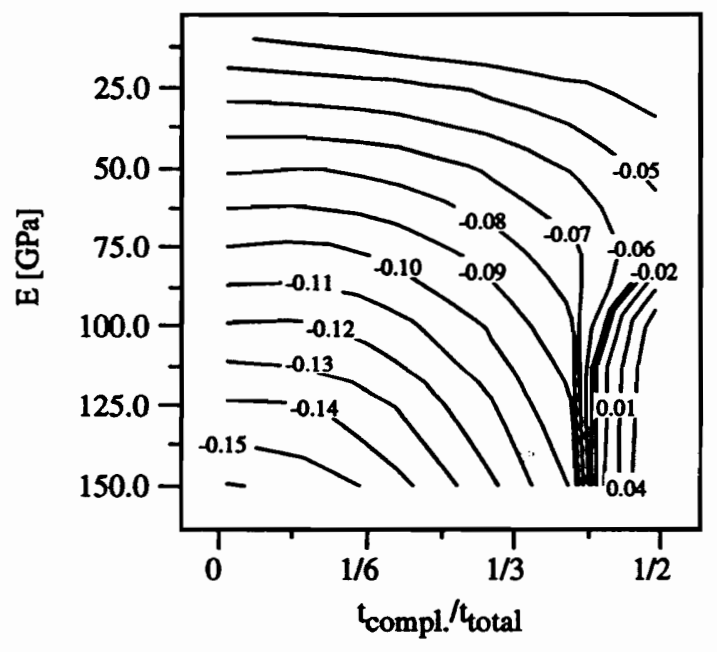

(c) $\bar{\sigma}_{z(\max )}$

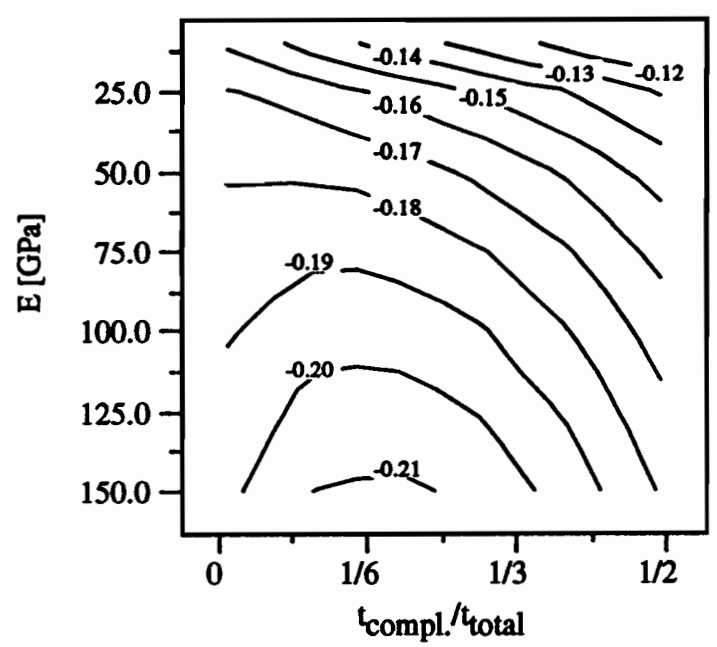

(b) $\bar{\sigma}_{n(\max )}$

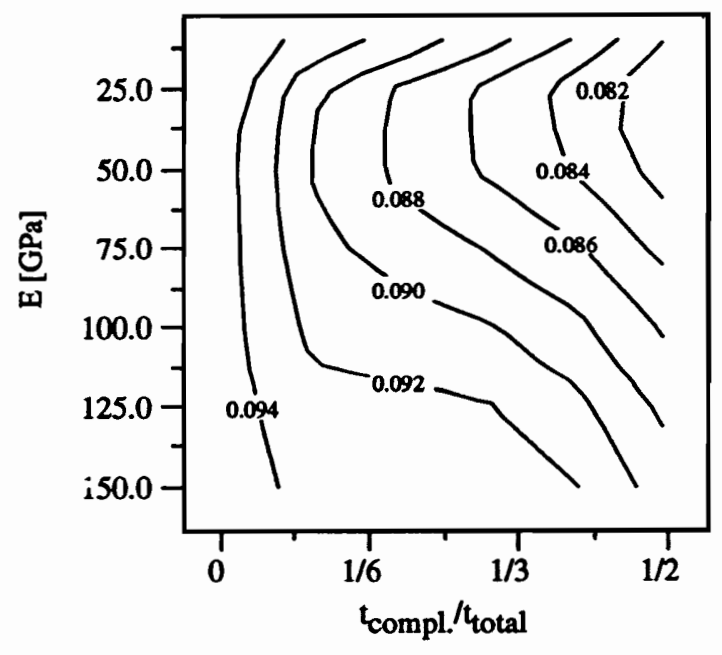

(d) $\bar{\tau}_{\operatorname{mn}(\max )}$

Figure 6.29: Effect of thickness and quasi-isotropic moduli of the compliant coating on the maximum stresses in the compliant coating. 


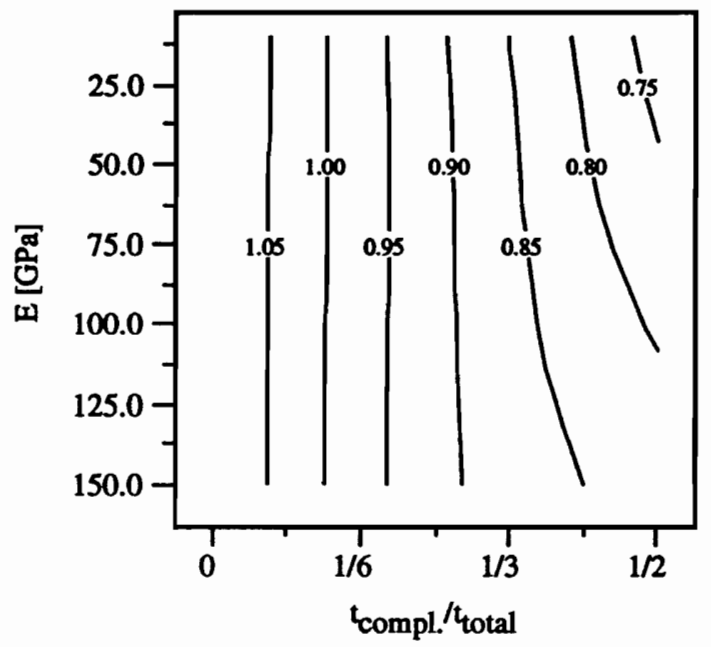

(a) $\bar{\sigma}_{1(\max )}$

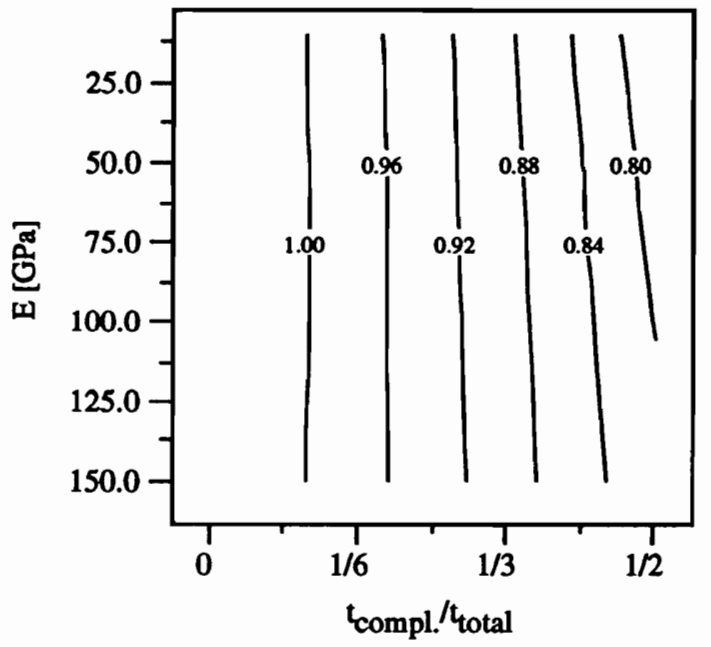

(c) $\bar{\sigma}_{z(\max )}$

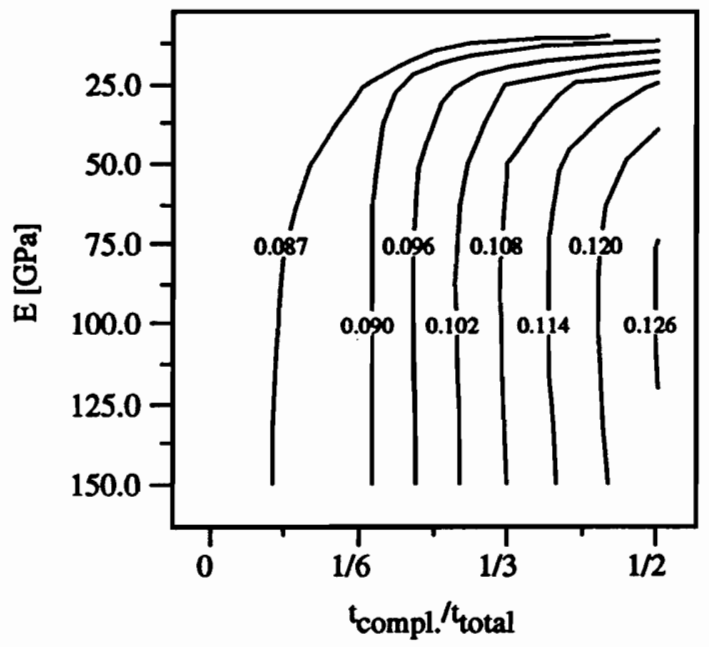

(b) $\bar{\sigma}_{2(\max )}$

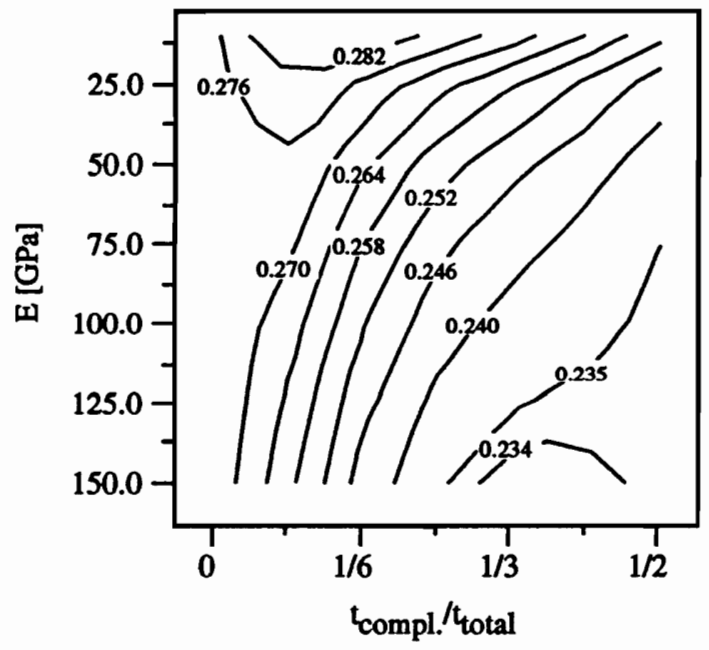

(d) $\bar{\tau}_{12(\max )}$

Figure 6.30: Effect of thickness and quasi-isotropic moduli of the compliant coating on the maximum stresses in the $\mathrm{C} / \mathrm{C}$ substrate. 


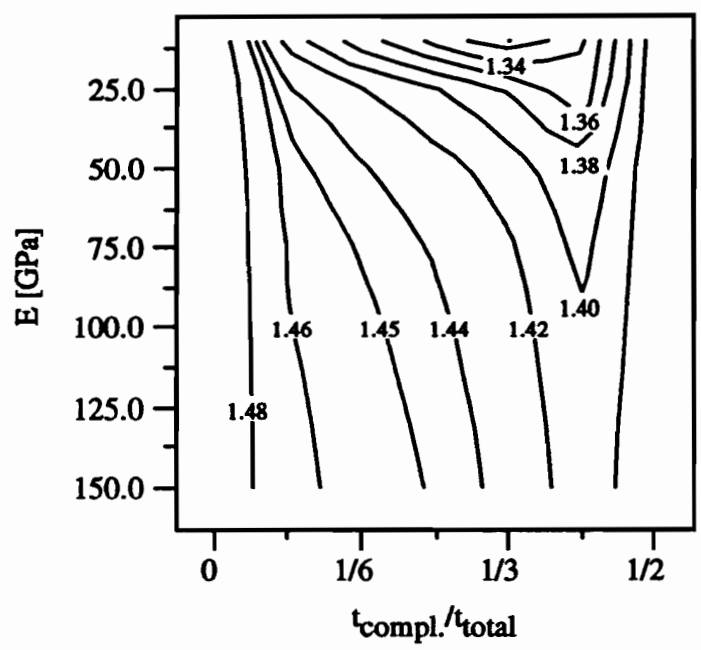

(a) $\bar{\sigma}_{e(\max )}$

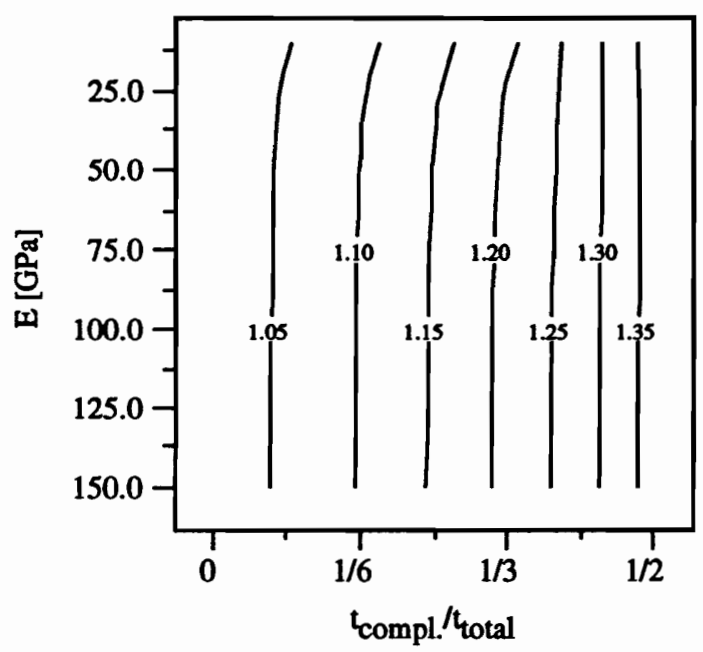

(c) $\bar{\sigma}_{z(\max )}$

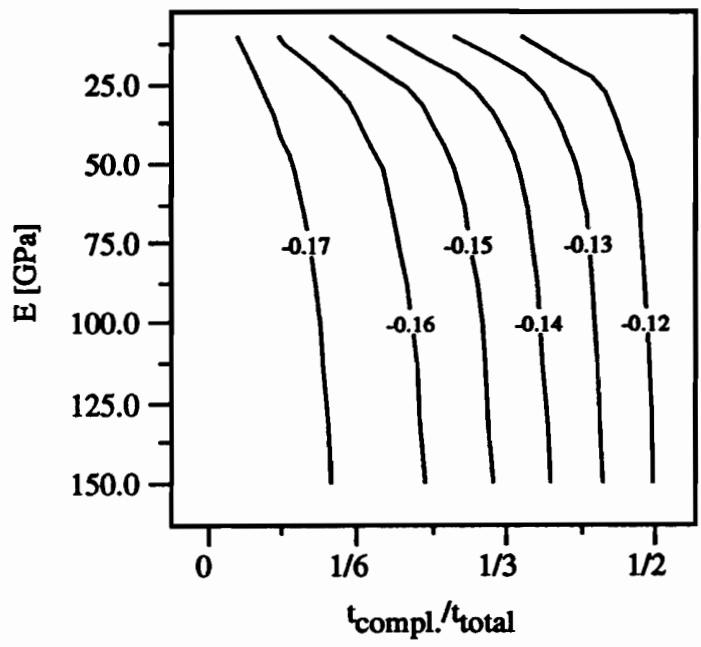

(b) $\bar{\sigma}_{n(\max )}$

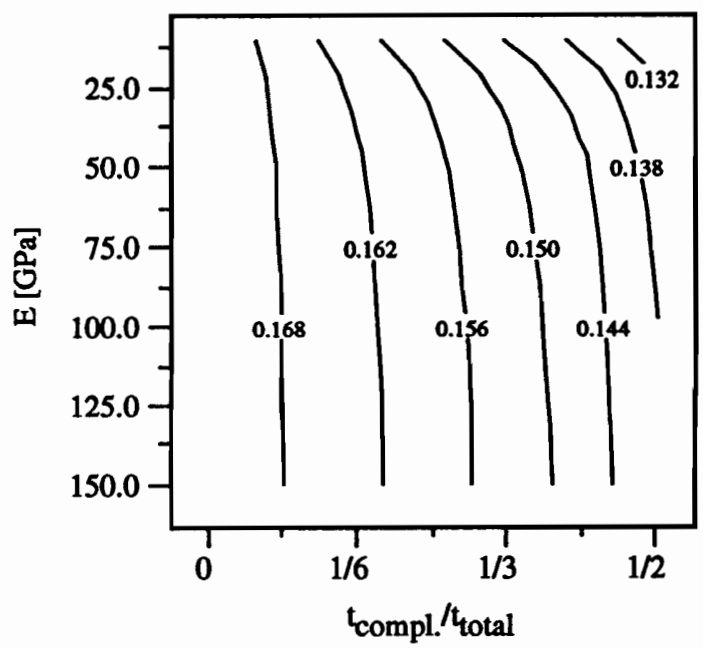

(d) $\bar{\tau}_{\operatorname{sn}(\max )}$

Figure 6.31: Effect of thickness and transverse modulus of the compliant coating on the maximum stresses in the $\mathrm{SiC}$ coating. 


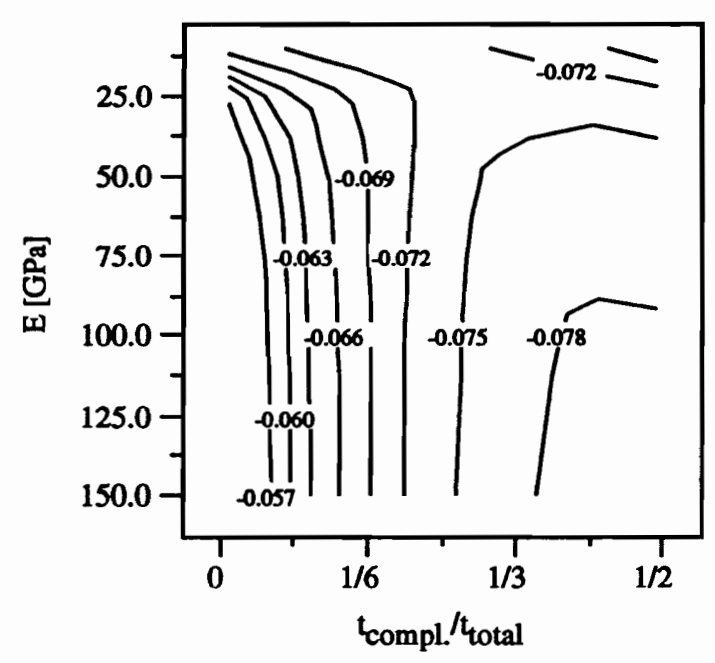

(a) $\bar{\sigma}_{\text {(max) }}$

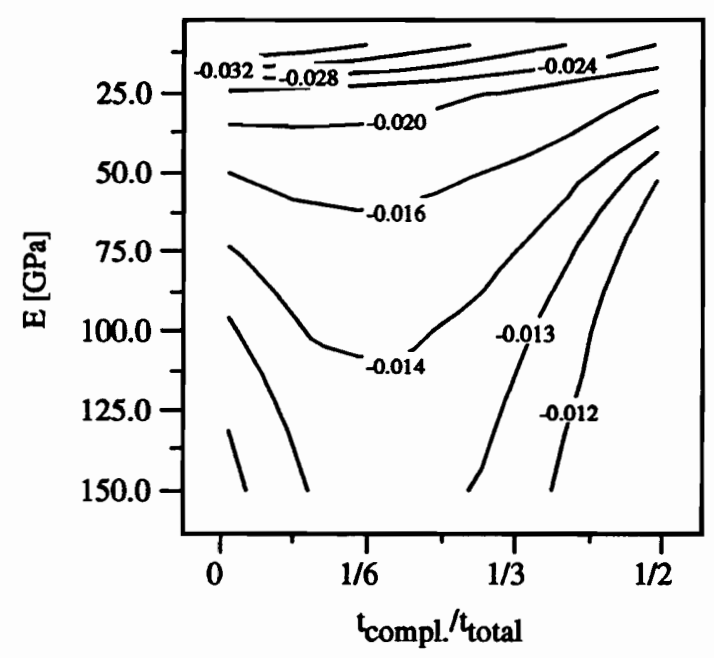

(c) $\bar{\sigma}_{z(\max )}$

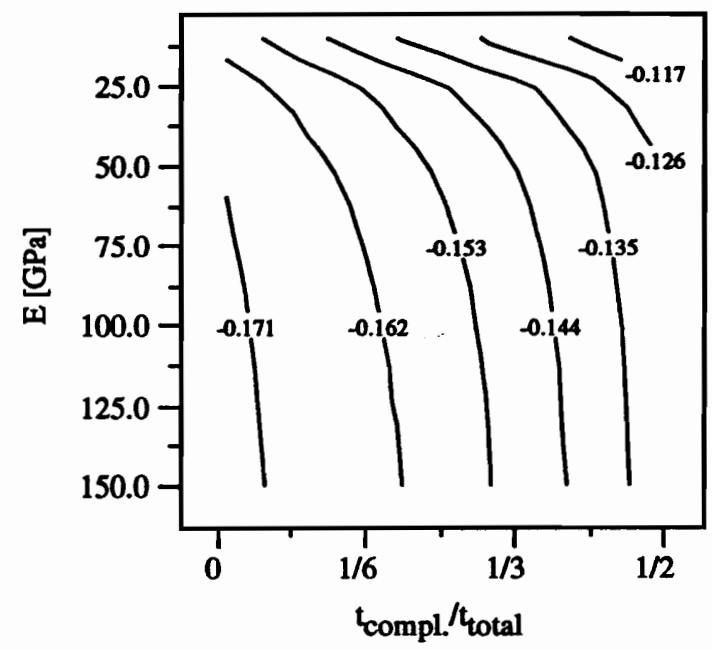

(b) $\bar{\sigma}_{n(m e x)}$

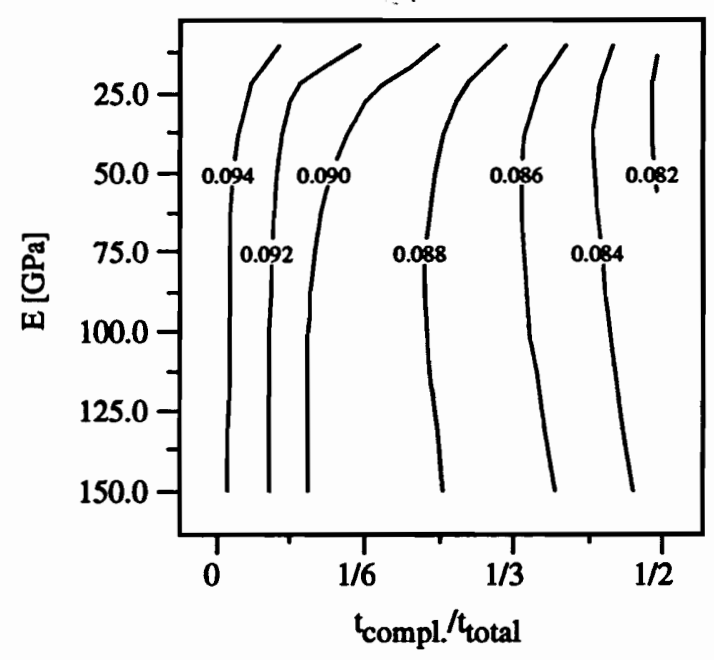

(d) $\bar{\tau}_{s n(\max )}$

Figure 6.32: Effect of thickness and transverse modulus of the compliant coating on the maximum stresses in the compliant coating. 


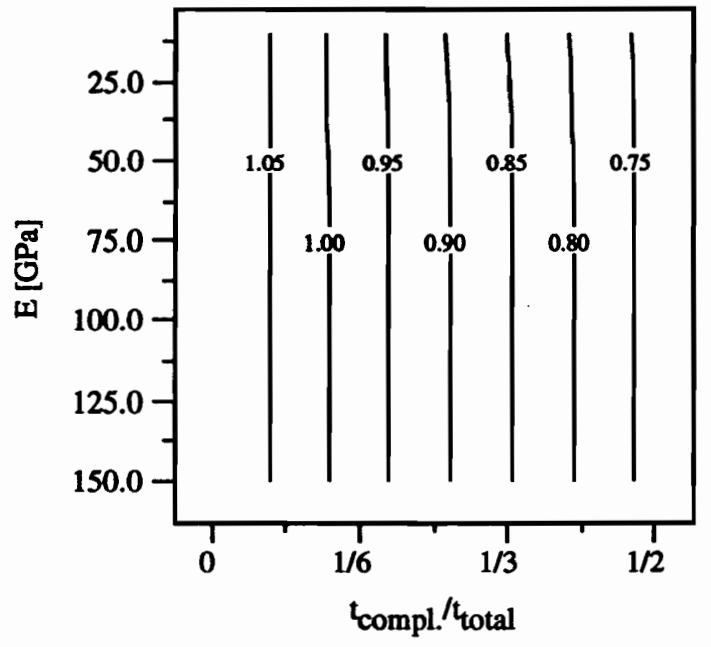

(a) $\bar{\sigma}_{1(\max )}$

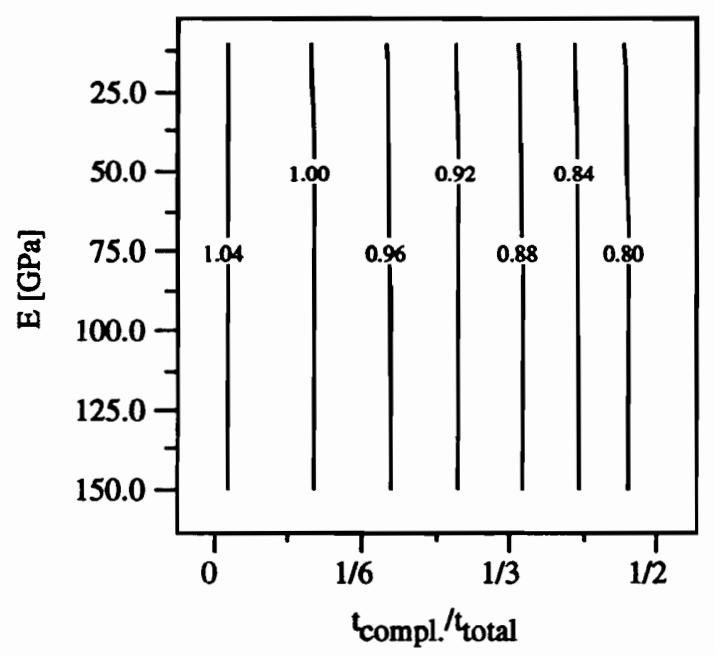

(c) $\bar{\sigma}_{x(\text { mex })}$

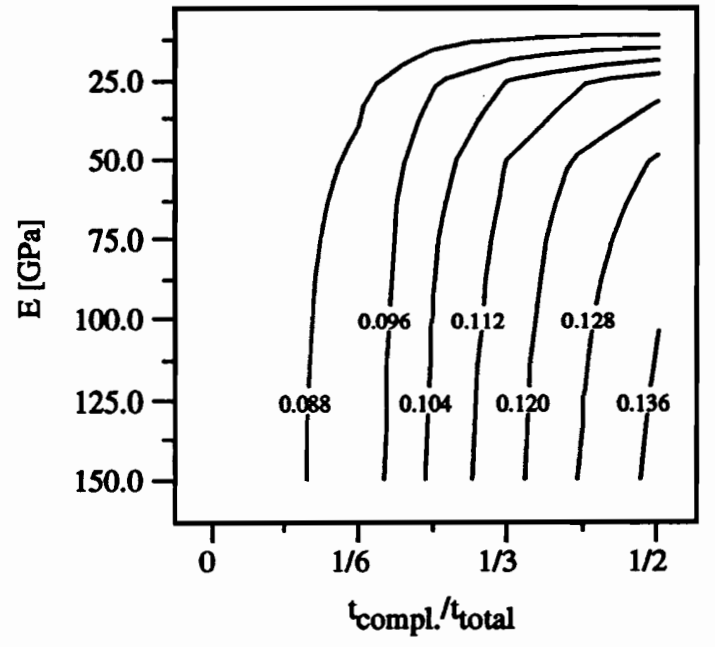

(b) $\bar{\sigma}_{2(\max )}$

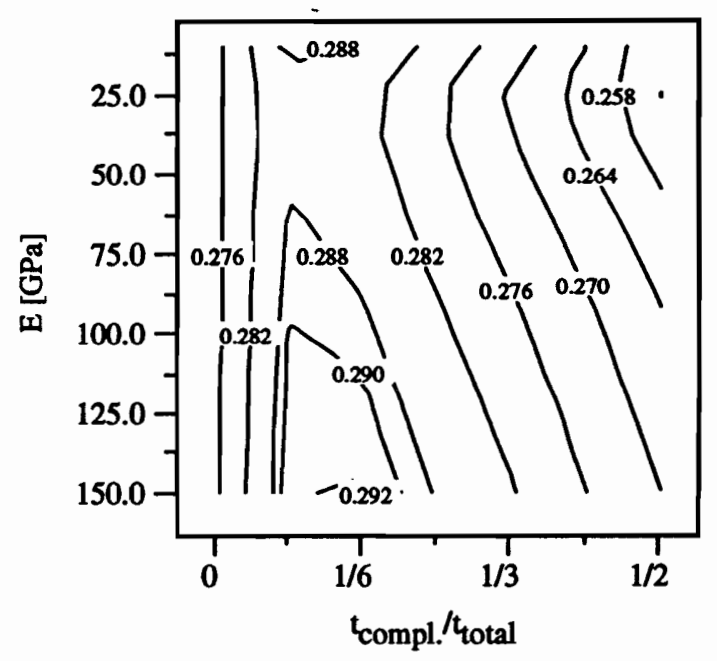

(d) $\bar{\tau}_{12(\max )}$

Figure 6.33: Effect of thickness and transverse modulus of the compliant coating on the maximum stresses in the $\mathrm{C} / \mathrm{C}$ substrate. 


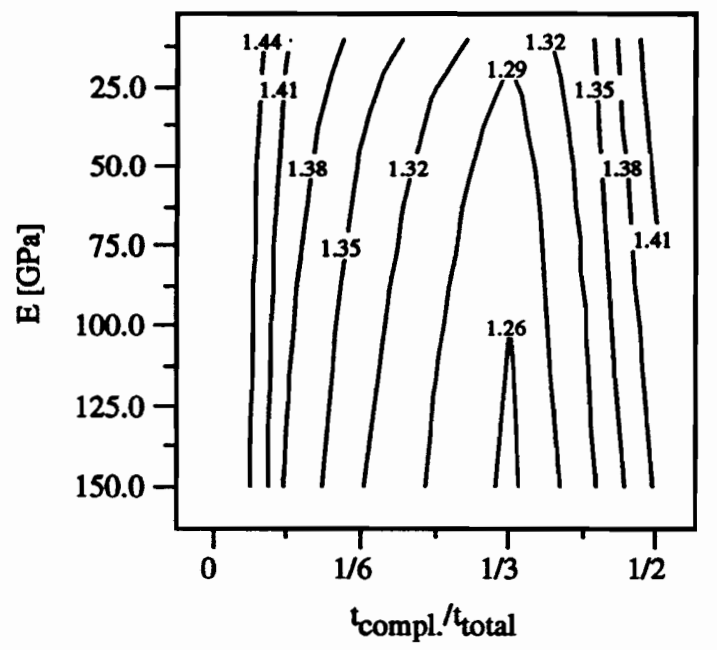

(a) $\bar{\sigma}_{s(\max )}$

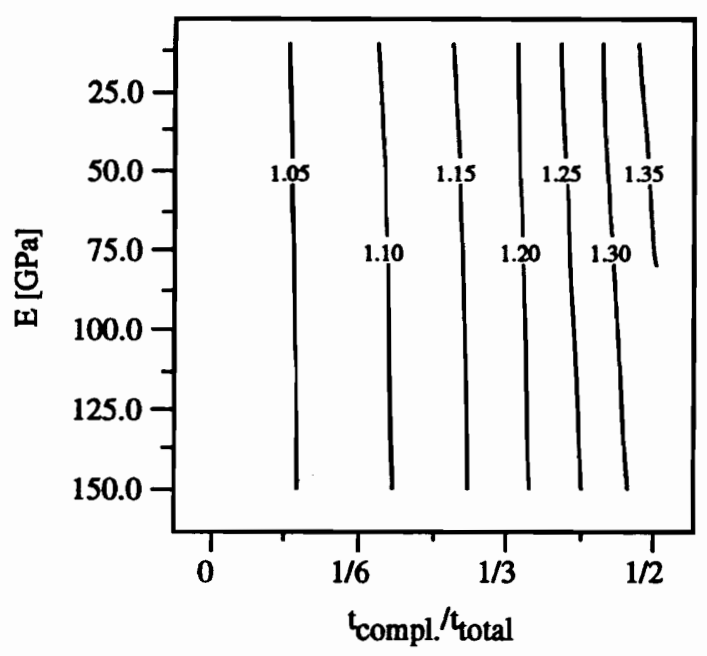

(c) $\overline{\boldsymbol{\sigma}}_{\boldsymbol{z}(\max )}$

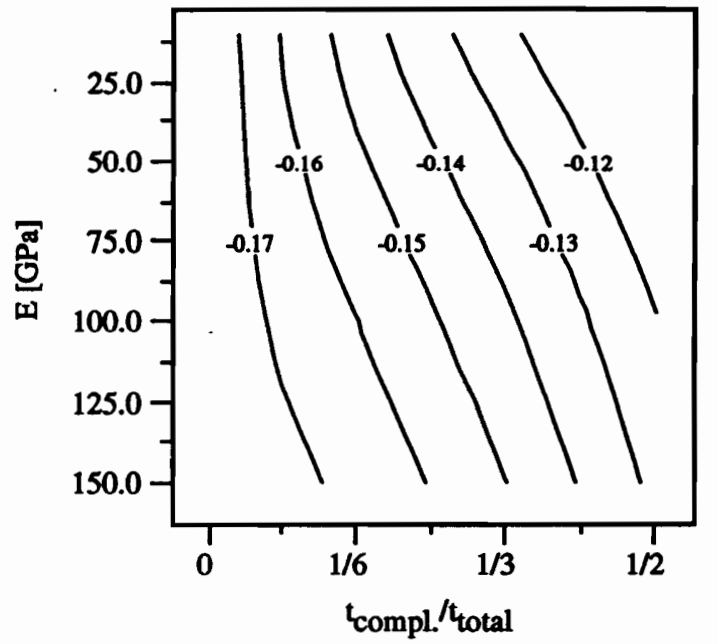

(b) $\bar{\sigma}_{n(\max )}$

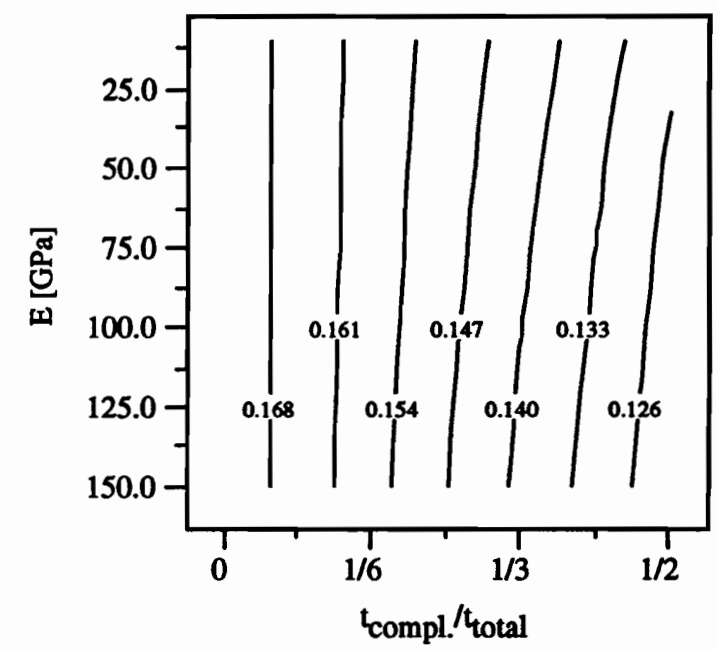

(d) $\bar{\tau}_{\operatorname{sn}(\max )}$

Figure 6.34: Effect of thickness and inplane moduli of the compliant coating on the maximum stresses in the $\mathrm{SiC}$ coating. 


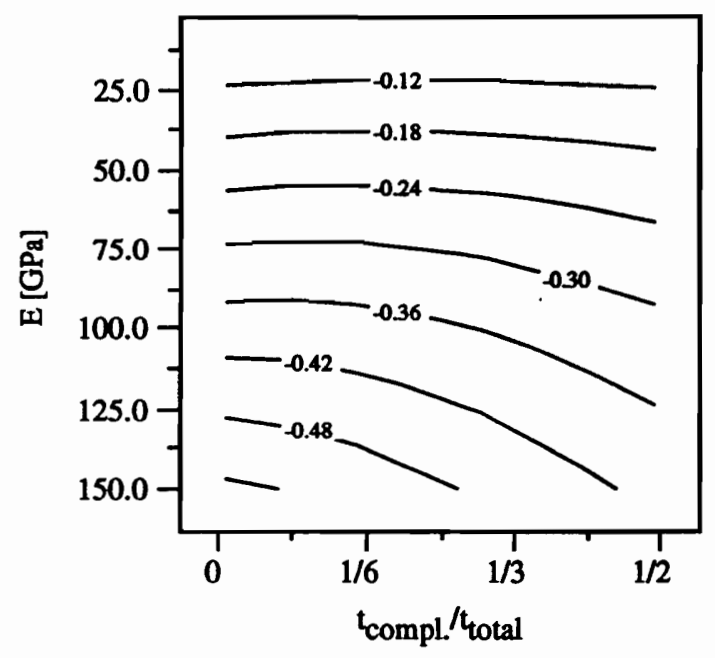

(a) $\bar{\sigma}_{e(\operatorname{mex})}$

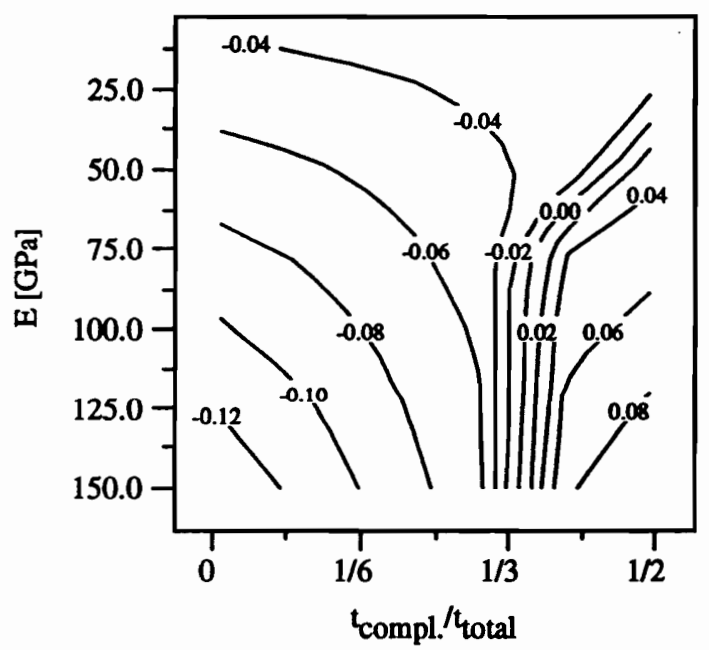

(c) $\bar{\sigma}_{x(\max )}$

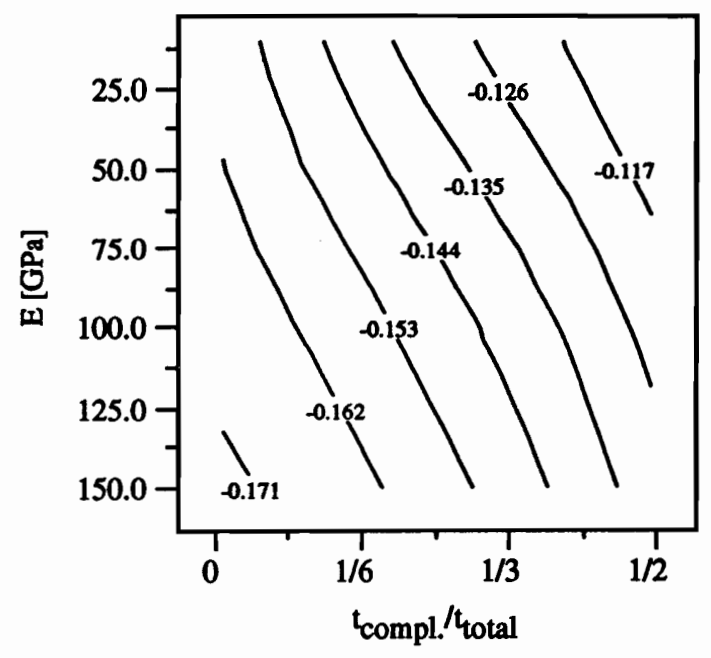

(b) $\bar{\sigma}_{n(\max )}$

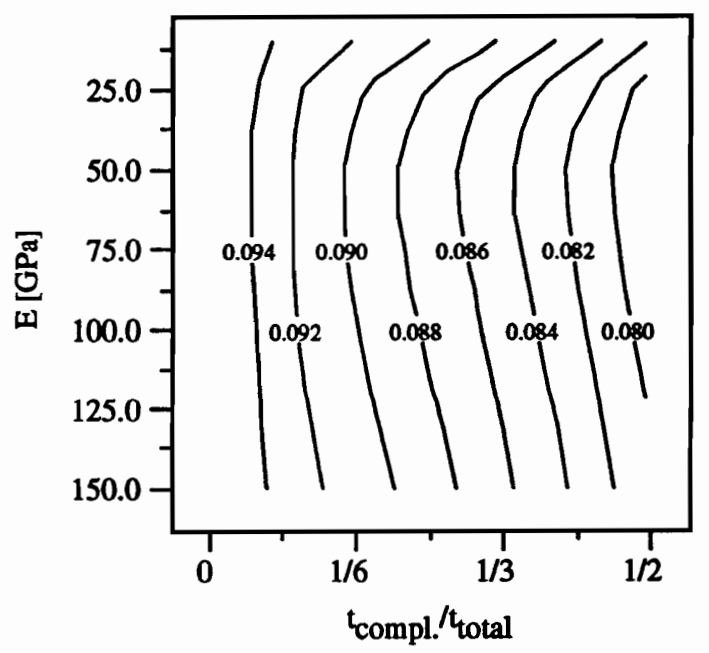

(d) $\tilde{\tau}_{\operatorname{sn}(\max )}$

Figure 6.35: Effect of thickness and inplane moduli of the compliant coating on the maximum stresses in the compliant coating. 


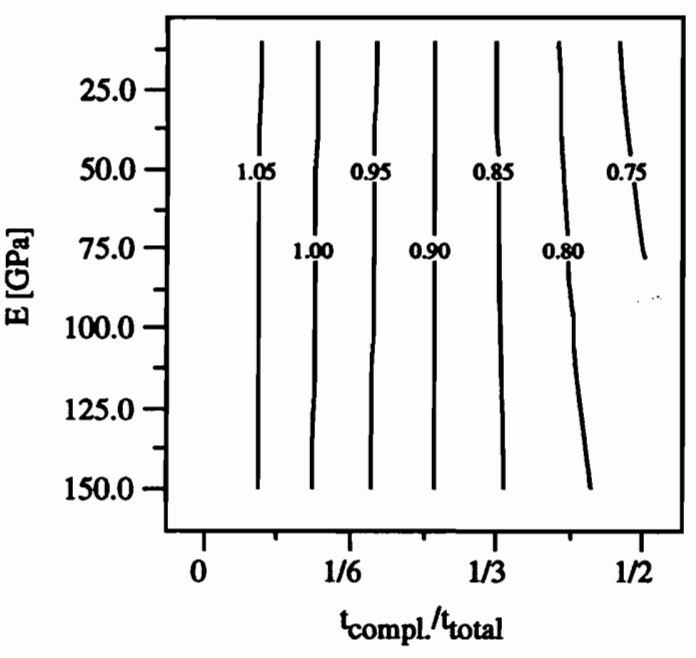

(a) $\bar{\sigma}_{1(\operatorname{mex})}$

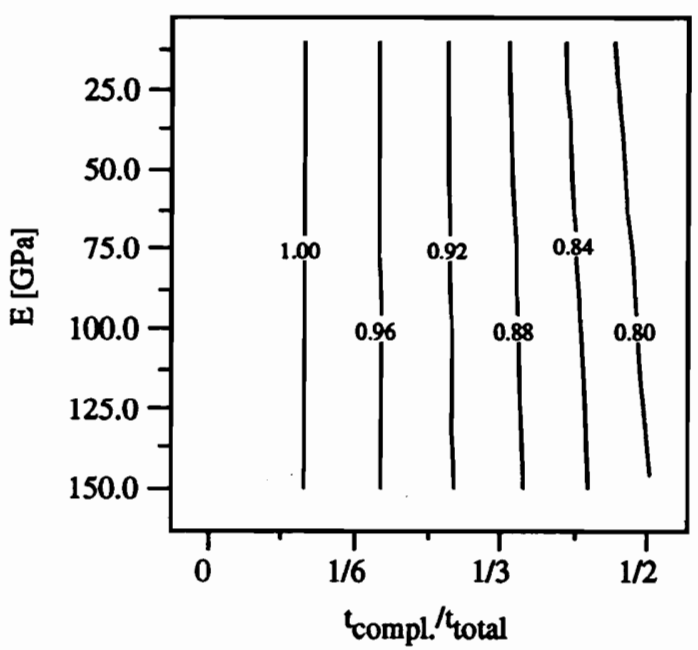

(c) $\bar{\sigma}_{x(m a x)}$

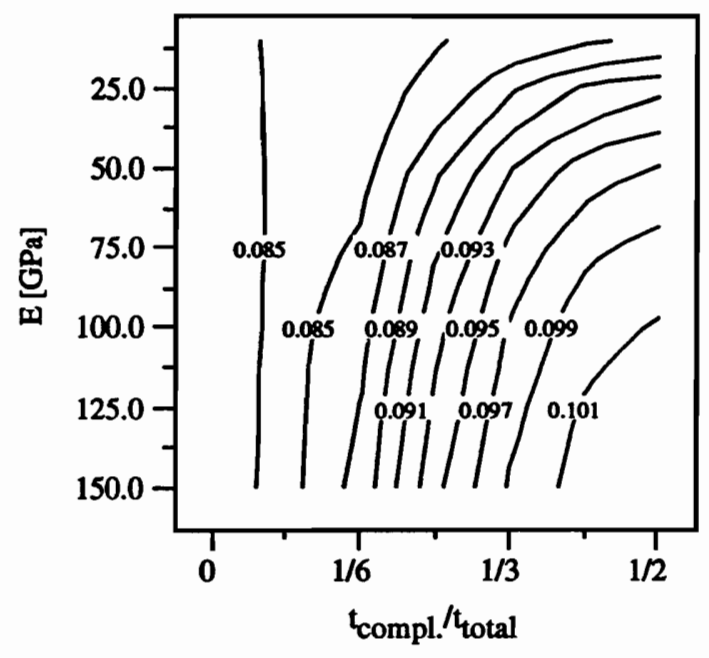

(b) $\bar{\sigma}_{2(\max )}$

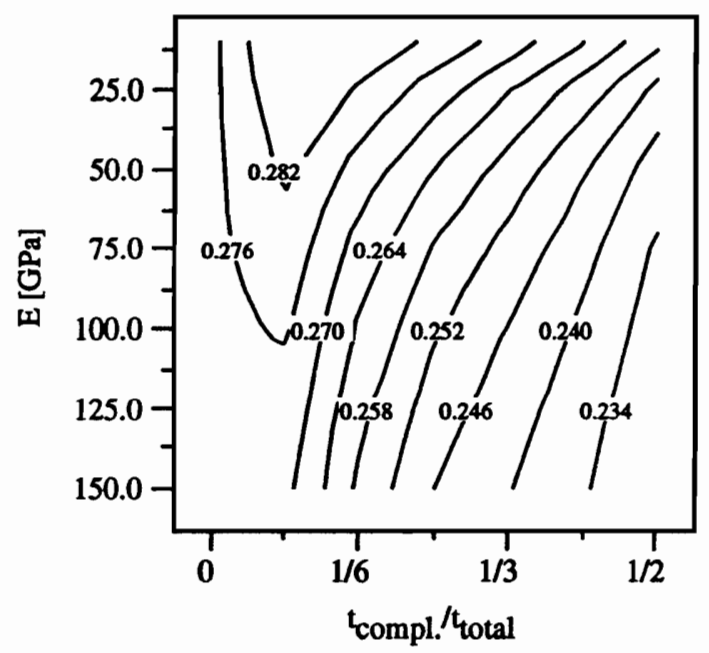

(d) $\bar{\tau}_{12(\max )}$

Figure 6.36: Effect of thickness and inplane moduli of the compliant coating on the maximum stresses in the $\mathrm{C} / \mathrm{C}$ substrate. 
coating increased significantly (Fig. 6.20b).

- For large and small coefficients of thermal expansion of the compliant coating, an increase of the modulus of the compliant coating resulted in significantly increased stresses in the compliant coating. In fact, stress levels were such that the compliant coating might fail before the $\mathrm{SiC}$ coating (Fig. 6.20b).

- No further improvement in stress levels in the $\mathrm{SiC}$ coating was observed for orthotropic compliant coatings compared to the case of an isotropic compliant coating (Fig. 6.20b). In fact, for a quasi-isotropic or a transverse increase of the modulus of elasticity of the compliant coating, the maximum tangential stress in the $\mathrm{SiC}$ coating actually increased with increasing modulus of the compliant coating (Figs. 6.28 and 6.31a).

- Optimum thicknesses of the compliant coating may exist for which the maximum tangential stress in the $\mathrm{SiC}$ coating has its smallest value. For smaller thicknesses, the maximum stress occurs in the stress concentration in the corner region, and for larger thicknesses the maximum stress occurs in the far field at the vertical line of symmetry (Fig. 6.11).

- Large shear deformation in the corner region in the compliant coating may cause a significant reduction in the stress concentration for all stress components in the $\mathrm{SiC}$ coating.

- The stresses in the substrate are only moderately sensitive to any of the material property variations and the thickness variation of the compliant coating. Thus, failure of the substrate is not expected due to any tailoring of compliant coating material properties.

From these observations it appears that an compliant coating with a high coefficient of thermal expansion, a low modulus, and an intermediate thickness results in the lowest stresses in the $\mathrm{SiC}$ coating. For this combination of parameters, the stresses in the compliant coating and the substrate are not adversely affected. 


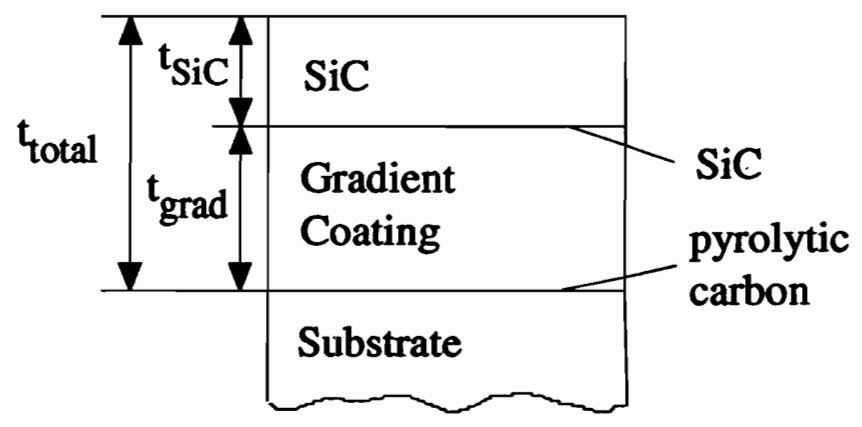

Figure 6.37: Geometry of a $\mathrm{C} / \mathrm{C}$ substrate with a gradient coating and a $\mathrm{SiC}$ coating.

\subsection{Gradient Coating Concept for Stress Reduction}

\subsubsection{Linear Pyrolytic Carbon to SiC Gradient Coating}

In Section 6.4.1 the influence of the thickness of a pure pyrolytic carbon layer between the $\mathrm{C} / \mathrm{C}$ substrate and the $\mathrm{SiC}$ coating was studied. It was found that there is a thickness of the pyrolytic carbon layer for which the lowest stress in the $\mathrm{SiC}$ coating is obtained. In this section a similar study is described, in which the pure pyrolytic carbon coating was replaced by a gradient coating, the gradient properties changing linearly from those of pyrolytic carbon at the gradient coating-substrate interface to those of $\mathrm{SiC}$ at the gradient coating-SiC interface. The thickness of the gradient coating was varied, while the total coating thickness was kept constant. The coating geometry is indicated in Fig. 6.37. All stresses were calculated at $20^{\circ} \mathrm{C}$ and normalized by the values given in Table 6.1.

In Table 6.4 the normalized maximum values of the $s-n$ stress components are given for different thicknesses of the gradient coating. It should be noted that the first column in Table 6.3 is identical to the first column in Table 6.4, i.e., to the case of no inner coating. As can be seen in Table 6.4, the change of the maximum stresses in the SiC coating and in the $\mathrm{C} / \mathrm{C}$ substrate with increasing gradient coating thickness is similar to that described for an increasing thickness pure pyrolytic carbon coating (Table 6.3). From Table 6.4 it can be seen that a thickness exists at which the tangential stress in the $\mathrm{SiC}$ coating reaches a 
minimum. For the gradient coating this thickness is at about $t_{\text {grad }} / t_{\text {total }}=1 / 4$, and the tangential stress is less than for the case of the pure pyrolytic carbon coating, i.e., 1.1299 vs. 1.2998. As shown in Fig. 6.38, for this thickness the far field tangential stress at the vertical line of symmetry and the maximum tangential stress in the stress concentration have the same magnitude. For smaller thicknesses, the maximum tangential stress occurs in the stress concentration region, and for larger thicknesses the maximum tangential stress occurs in the far field. This is very similar to the situation in Fig. 6.11 for the discrete pyrolytic carbon and $\mathrm{SiC}$ coatings. There at $t_{\text {grad }} / t_{\text {total }}=1 / 3$ the lowest magnitude maximum stress in the $\mathrm{SiC}$ occured when the tangential stress at the stress concentration and in the far field were equal. For the $\mathrm{SiC}$ coating, the maximum tangential stress in the stress concentration is at the $\mathrm{SiC}$-gradient coating interface, whereas the maximum tangential stress in the far field occurs near the free surface. The maximum tangential stress in the gradient coating occurs near the gradient coating-SiC interface for all gradient coating thicknesses, because near this interface the gradient coating has properties close to the stiff SiC properties.

The maximum normalized mechanical strains in the $s-n$ coordinate system for the coatings and in the principal material direction coordinate system for the substrate for different gradient coating thicknesses are shown in Table 6.5. All strain components in the coatings were normalized by the far field tangential mechanical strain in the $\mathrm{SiC}$ coating calculated at $20^{\circ} \mathrm{C}$ using the ISPSA for the baseline case. Similarly, all strain components in the substrate were normalized by the far field tangential mechanical strain in the substrate calculated at $20^{\circ} \mathrm{C}$ using the ISPSA for ihe baseline case. These mechanical strains were $\epsilon_{s, S i C}=0.002089$ and $\epsilon_{s, S u b s t r a t e}=-0.003464$. In the $\mathrm{C} / \mathrm{C}$ substrate all strains vary with the gradient coating thickness in the same way as the stresses shown in Table 6.4. However, in the substrate the strain in the soft 2-direction and the shear strain are larger than the strain in the stiff 1 - and $z$-directions.

In the $\mathrm{SiC}$ coating all strains except the normal strain $\bar{\epsilon}_{n}$ vary in the same way as the stresses. The normal strain reaches a minimal value at the same gradient coating thickness as the tangential strain. In contrast, the normal stress decreases for increasing gradient 


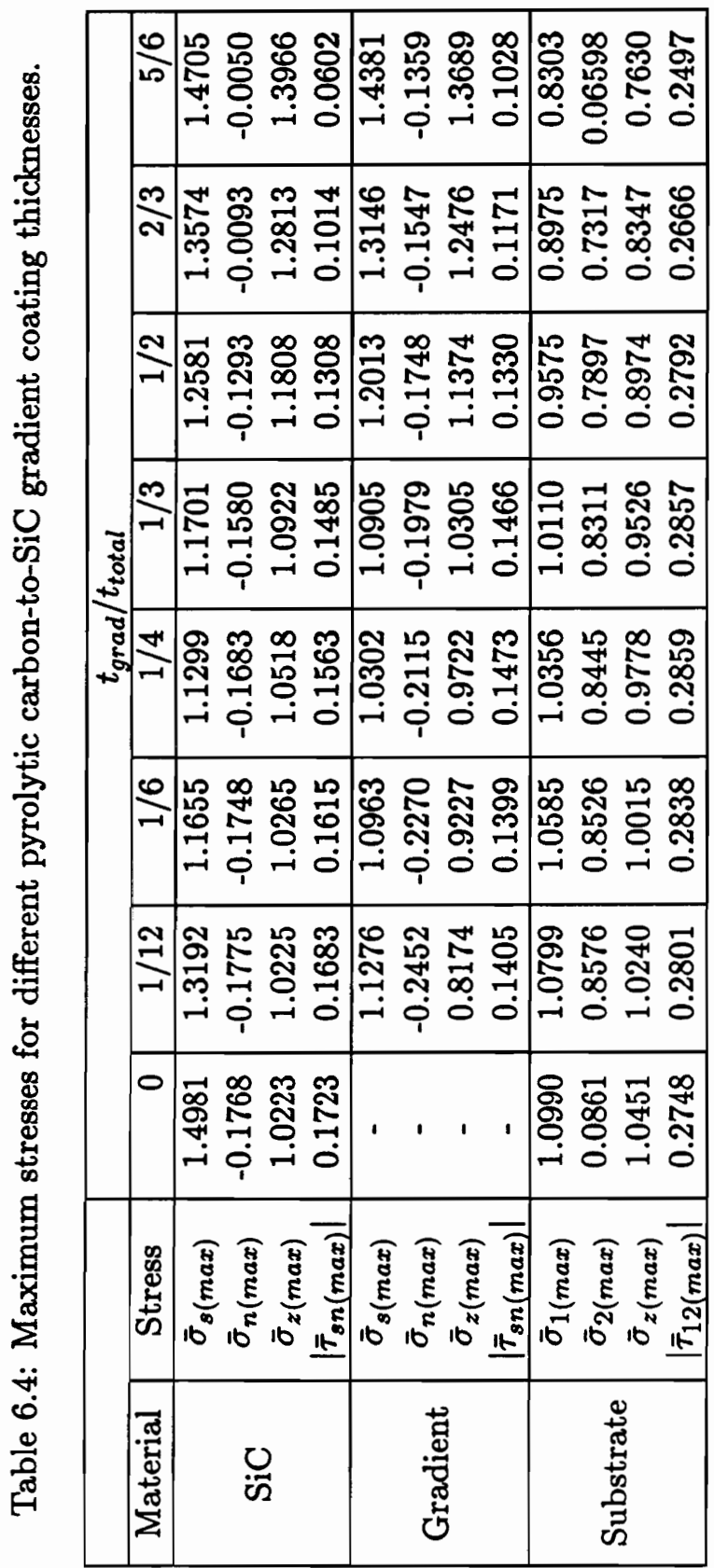




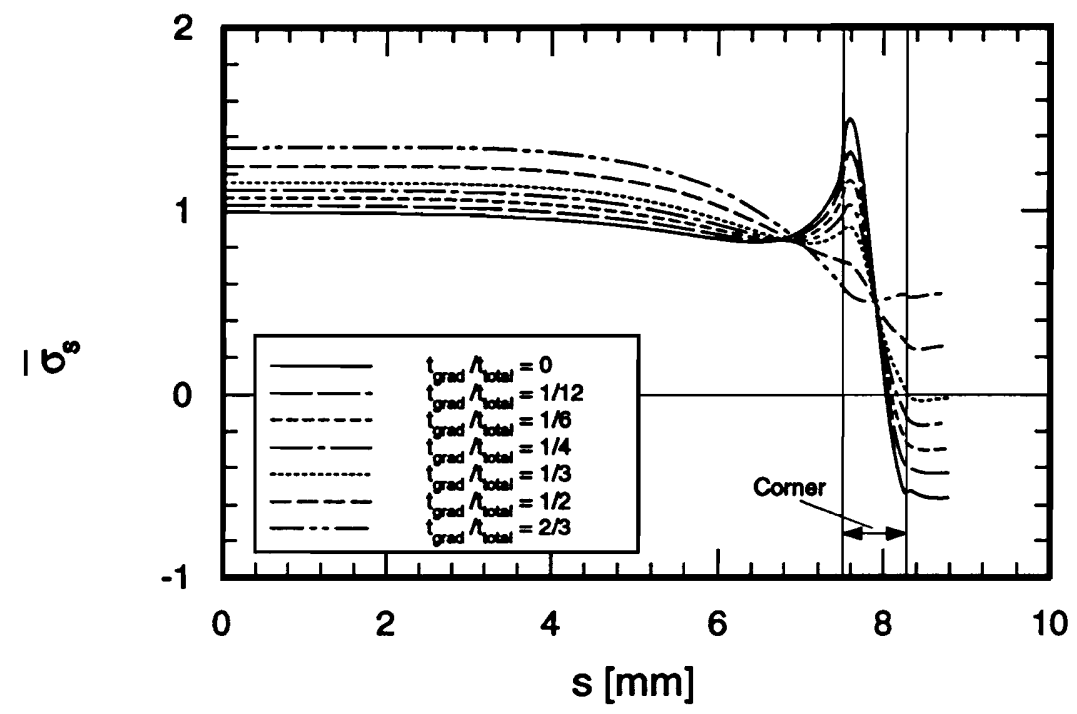

Figure 6.38: Tangential stress in $\mathrm{SiC}$ coating near interface for different gradient coating thicknesses. 
coating thicknesses.

The maximum strains in the gradient coating vary differently with thickness than the maximum stresses. While the maximum stresses occur at the gradient coating-SiC interface, the maximum strains occur at the gradient coating-substrate interface in the corner region. The maximum tangential strain reaches a maximum value at $t_{\text {grad }} / t_{\text {total }}=1 / 2$. The maximum magnitude of the tangential strain in the gradient coating is about twice the strain for an infinitely wide substrate. The maximum normal strain increases in magnitude with increasing gradient coating thickness. The shear strain near the substrate-gradient coating interface is the largest of all strain components. This supports the conclusions drawn in Section 6.4 that large shear deformations in a softer coating between the $\mathrm{SiC}$ coating and the substrate can reduce the stress concentrations in the $\mathrm{SiC}$ coating. Because the shear strains in the gradient coating reach large values, both a strain failure criterion and a stress failure criterion should be used to assess failure of the gradient coating.

\subsubsection{Variations of the Gradient Coating Profiles}

In the previous section it was shown that using a linear variation of properties from pyrolytic carbon to $\mathrm{SiC}$ in the inner coating resulted in lower stresses in the $\mathrm{SiC}$ coating than using a pure pyrolytic carbon coating. In this section it is discussed how different nonlinear variations of the properties through the thickness of the gradient coating influence the maximum stresses in the $\mathrm{SiC}$ coating. For this purpose a concave and a convex distribution of properties inrough the thickness of the coating were compared to the linear distribution. This was done for a gradient coating thickness of $t_{\text {grad }} / t_{\text {total }}=1 / 3$. Both nonlinear gradient distributions are described by exponential functions. The equation for the concave distribution of the properties through the thickness of the gradient coating is

$$
\mathcal{P}_{g}(t)=\mathcal{P}_{c} \exp \left[\frac{t}{t_{g}} \ln \left(\frac{\mathcal{P}_{S i C}}{\mathcal{P}_{c}}\right)\right]
$$

and the equation for the convex distribution is

$$
\mathcal{P}_{g}(t)=\mathcal{P}_{c}+\mathcal{P}_{S i C}+\mathcal{P}_{c} \exp \left[\frac{\left(t_{g}-t\right)}{t_{g}} \ln \left(\frac{\mathcal{P}_{S i C}}{\mathcal{P}_{c}}\right)\right]
$$




\begin{tabular}{|c|c|c|c|c|}
\hline & $\frac{0}{10}$ & 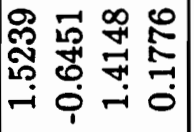 & 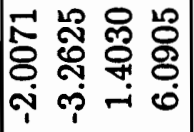 & 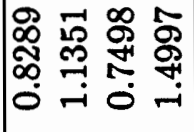 \\
\hline & $\frac{\infty}{N}$ & 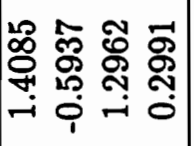 & 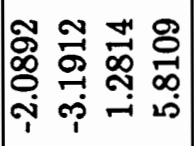 & 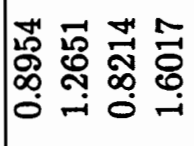 \\
\hline & $\stackrel{N}{-}$ & 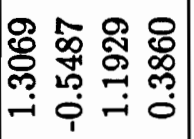 & 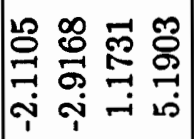 & 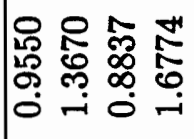 \\
\hline تัّ & $\stackrel{m}{-1}$ & 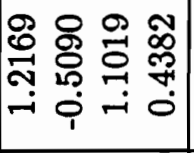 & 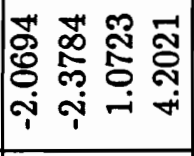 & 跑 \\
\hline , & & 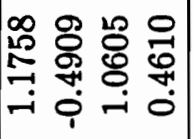 & 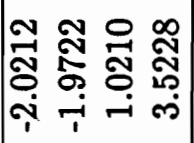 & 乍 \\
\hline & 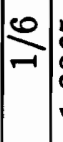 & 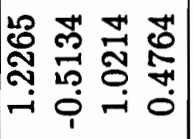 & 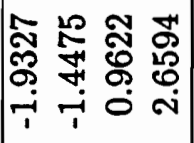 & 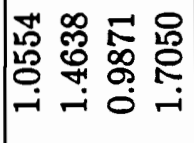 \\
\hline & $\stackrel{\sim}{=}$ & 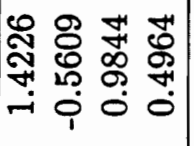 & 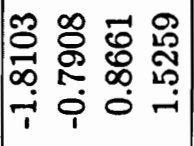 & 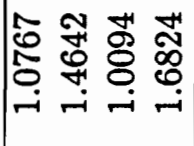 \\
\hline & 0 & 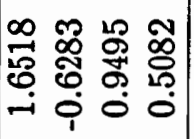 & 1,1 & 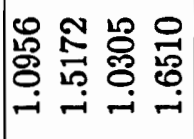 \\
\hline & 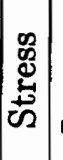 & 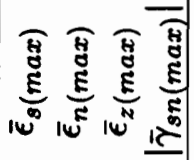 & 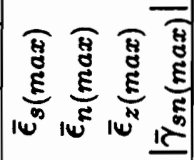 & 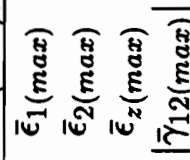 \\
\hline & 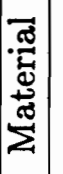 & బூ & 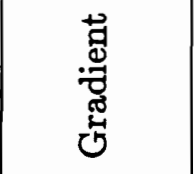 & 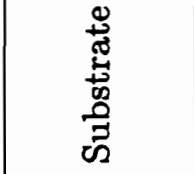 \\
\hline
\end{tabular}




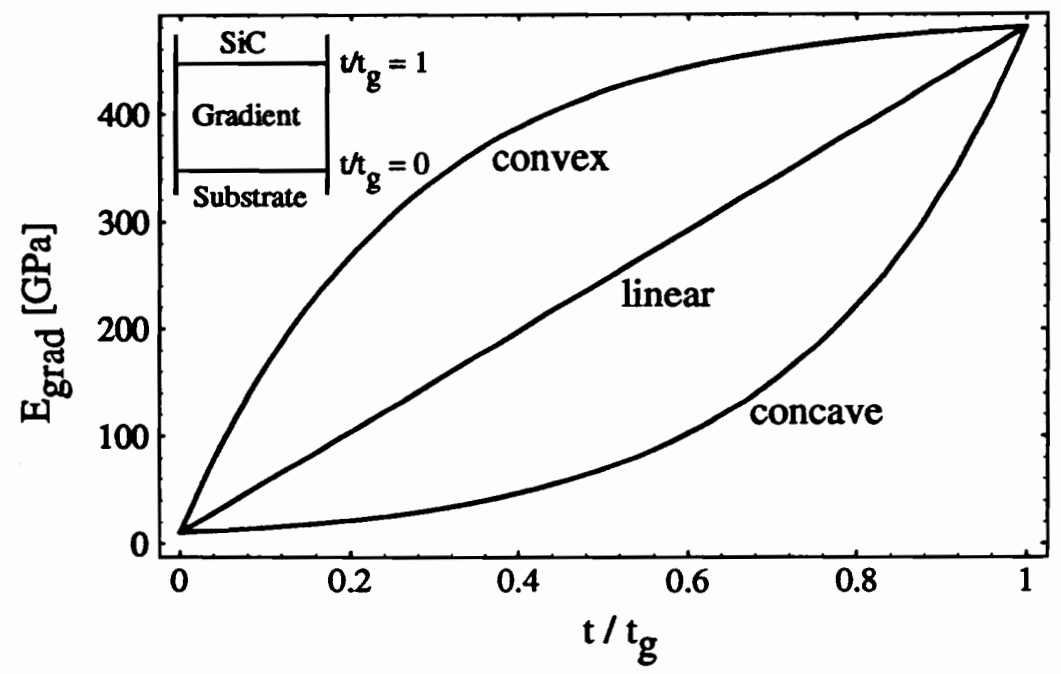

Figure 6.39: Variation of properties through a gradient coating.

As a comparison, the linear variation of the properties through the gradient is given by

$$
\mathcal{P}_{g}(t)=\mathcal{P}_{c}+\frac{t}{t_{g}}\left(\mathcal{P}_{S i C}-\mathcal{P}_{c}\right)
$$

Here $t_{g}$ denotes the thickness of the gradient coating, $t$ is the thickness coordinate which is zero at the gradient coating-substrate interface and increases outward towards the SiC coating (see Fig. 6.37), and $\mathcal{P}$ represents any property. The subscripts $g, c$, and $S i C$ on $\mathcal{P}$ denote the gradient coating, the pyrolytic carbon coating, and the $\mathrm{SiC}$ coating, respectively. As an example, the variation of the modulus of elasticity is shown for the concave, the linear, and the convex functions in Fig. 6.39. In the current implementation of the finite element program nonlinear gradients are approximated by a sequence of linear gradients. The quality of the approximation therefore depends on the number of the finite elements through the thickness of the gradient coating. In the present study for $t_{\text {grad }} / t_{\text {total }}=1 / 3$ eight elements were used through the thickness of the gradient coating.

The maximum stresses in the $\mathrm{SiC}$ coating, the gradient coating, and the substrate for the three gradient distributions are given in Table 6.6. For all stress components the magnitudes of stresses for the linear gradient coating are between the magnitudes of the stresses for the 
Table 6.6: Maximum stresses for different material property distributions through-thethickness of the gradient coating.

\begin{tabular}{|c|c|c|c|c|c|}
\hline & & $\bar{\sigma}_{s, \max }$ & $\bar{\sigma}_{n, \max }$ & $\bar{\sigma}_{z, \max }$ & $\left|\bar{\tau}_{s n, \max }\right|$ \\
\hline \multirow{3}{*}{$\mathrm{SiC}$} & Concave & 1.210 & -0.096 & 1.139 & 0.131 \\
\hline & Linear & 1.170 & -0.159 & 1.092 & 0.150 \\
\hline & Convex & 1.132 & -0.189 & 1.056 & 0.155 \\
\hline \multirow{3}{*}{ Gradient } & Concave & 1.126 & -0.145 & 1.065 & 0.131 \\
\hline & Linear & 1.120 & -0.183 & 1.059 & 0.148 \\
\hline & Convex & 1.099 & -0.189 & 1.039 & 0.155 \\
\hline & & $\bar{\sigma}_{1, \max }$ & $\bar{\sigma}_{2, \max }$ & $\bar{\sigma}_{z, \max }$ & $\left|\bar{\tau}_{12, \max }\right|$ \\
\hline \multirow{3}{*}{ Substrate } & Concave & 0.969 & 0.082 & 0.926 & 0.301 \\
\hline & Linear & 1.012 & 0.083 & 0.953 & 0.287 \\
\hline & Convex & 1.031 & 0.083 & 0.975 & 0.273 \\
\hline
\end{tabular}

two nonlinear gradients. The maximum tangential stress in the SiC coating is the smallest for the convex gradient coating. As shown in Fig. 6.40, the maximum tangential stress in the SiC coating for all three gradient coating cases occurs in the far field. Because the convex gradient coating has the overall highest $\mathrm{SiC}$ content and hence is, on average, the stiffest coating, the maximum far field tangential stress for this case is lower than for the two other cases. It can be further seen in Fig. 6.40 that for the convex coating, the stress concentration in the tangential stress is the lowest. The axial stress in the SiC coating and the tangential and the axial stress in the gradient coating all are the smallest for the convex gradient case. In contrast, the normal and the shear stress for the convex gradient coating case are the largest. However, their magnitudes are small compared to the tangential and the axial stress and therefore the reduction in the magnitude of the tangential stress for the convex case outweighs the increase in normal and shear stress.

The principal material direction stress in the substrate in the fiber directions are the lowest for the concave gradient case, whereas the shear stress is the largest for this case.

A variation of the thickness of the convex gradient coating shows that as for the linear gradient coating, a thickness of the gradient coating exists, for which the maximum tangen- 


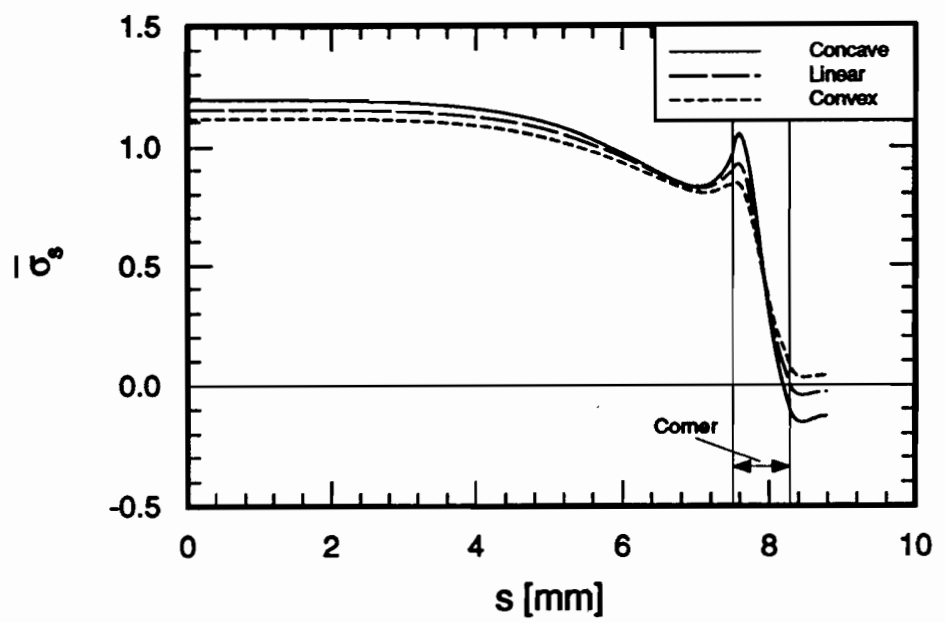

Figure 6.40: Tangential stress $\bar{\sigma}_{s}$ near the SiC-gradient coating in the SiC coating for different property distributions in the gradient coating.

Table 6.7: Effect of thickness of convex gradient coating on stresses in the SiC coating.

\begin{tabular}{|c|c|c|c|c|}
\hline$t_{\text {grad }} / t_{\text {total }}$ & $\bar{\sigma}_{s, \max }$ & $\bar{\sigma}_{n, \max }$ & $\bar{\sigma}_{z, \max }$ & $\left|\bar{\tau}_{s n, \max }\right|$ \\
\hline $1 / 3$ & 1.1701 & -0.1586 & 1.0922 & 0.1550 \\
$1 / 4$ & 1.1039 & -0.1830 & 1.0266 & 0.1610 \\
$1 / 6$ & 1.1193 & -0.1861 & 1.0078 & 0.1661 \\
\hline
\end{tabular}

tial stress in the $\mathrm{SiC}$ coating reaches its smallest value. In Table 6.7 the maximum stresses in the $\mathrm{SiC}$ coating are shown for three different thicknesses of the gradient coating. The tangential stress in the $\mathrm{SiC}$ coating is smallest for $t_{\text {grad }} / t_{\text {total }}=1 / 4$. While the change in the maximum tangential stress shown in Table 6.6 is less than $10 \%$ for the different gradient distributions for thin gradient coatings, it can be significantly larger for thick gradient coatings. To illustrate this point, a $\mathrm{C} / \mathrm{C}$ substrate with a pyrolytic carbon to $\mathrm{SiC}$ gradient coating with $t_{\text {grad }} / t_{\text {total }}=1$ was analyzed. All dimensions and properties were the same as described above. A relative gradient coating thickness of $t_{\text {grad }} / t_{\text {total }}=1$ corresponds to a substrate with only a gradient coating. There is no additional pure SiC coating. For this 
Table 6.8: Effect of gradient distribution on stresses in the coating and the substrate for thick gradient coatings.

\begin{tabular}{|c|c|c|c|c|c|}
\hline & & $\bar{\sigma}_{\boldsymbol{s}, \max }$ & $\bar{\sigma}_{n, \max }$ & $\bar{\sigma}_{z, \max }$ & $\mid \bar{\tau}_{s n, \max }$ \\
\hline \multirow{3}{*}{ Gradient } & Concave & 1.898 & -0.066 & 1.831 & 0.0588 \\
\hline & Linear & 1.588 & -0.108 & 1.588 & 0.0893 \\
\hline & Convex & 1.384 & -0.146 & 1.316 & 0.1130 \\
\hline & & $\bar{\sigma}_{1, \max }$ & $\bar{\sigma}_{2, \max }$ & $\bar{\sigma}_{z, \max }$ & $\mid \bar{\tau}_{12, \max }$ \\
\hline \multirow{3}{*}{ Substrate } & Concave & 0.5597 & 0.0451 & 0.4649 & 0.1815 \\
\hline & Linear & 0.7563 & 0.0576 & 0.6800 & 0.2311 \\
\hline & Convex & 0.8646 & 0.0646 & 0.8082 & 0.2402 \\
\hline
\end{tabular}

case, the maximum stresses for the concave, the linear, and the convex gradient distributions are shown for the gradient coating and the substrate in Table 6.8. It can be seen that the maximum tangential stress and the maximum axial stress are almost $40 \%$ smaller for the convex gradient distributions than for the concave gradient distribution. Since the normal and shear stresses are much smaller than the tangential and the axial stresses, the increase in those stresses for the convex gradient coating is not as significant as the decrease in the tangential and the axial stress. It is important to note, however, that the maximum values of $\bar{\sigma}_{s, \max }$ in Table 6.8 are higher than the maximum values of $\bar{\sigma}_{s, \max }$ in Table 6.6. This could be interpreted to mean an all-gradient coating is not as good as a gradient coating on top of which a $\mathrm{SiC}$ coating is deposited.

\subsubsection{Summary}

It has been shown in this section that the maximum stresses in the $\mathrm{SiC}$ coating can be reduced if a gradient coating is used instead of only a pure pyrolytic carbon coating. As for the pure pyrolytic carbon coating case, for the gradient coating case the thickness of the gradient coating should be optimized to obtain the lowest possible stresses in the $\mathrm{SiC}$ coating. A study of different distributions of properties through the thickness of the gradient coating showed that the tangential stress in the $\mathrm{SiC}$ coating was the lowest for a convex 
distribution.

\subsection{Multiple Coatings Applied at Different Temperatures}

Results discussed in the previous sections were for $\mathrm{C} / \mathrm{C}$ substrates with one or more coatings applied at the same temperature. As discussed in Section 1.3.2, often coatings are applied to the $\mathrm{C} / \mathrm{C}$ composites at different temperatures. In this section results from finite element analyses of $\mathrm{C} / \mathrm{C}$ substrates with discrete $\mathrm{B}_{4} \mathrm{C}$ and $\mathrm{Si}_{3} \mathrm{~N}_{4}$ coatings applied at different temperatures, and for $\mathrm{C} / \mathrm{C}$ substrates with a pyrolytic carbon- $\mathrm{B}_{4} \mathrm{C}-\mathrm{Si}_{3} \mathrm{~N}_{4}$ gradient coating applied with several different temperature scenarios are presented. In both cases the substrate has finite width and thus corner effects are included.

\subsubsection{Discrete Coatings}

In Section 6.1 an infinitely wide $\mathrm{C} / \mathrm{C}$ substrate with two coatings, $\mathrm{B}_{4} \mathrm{C}$ and $\mathrm{Si}_{3} \mathrm{~N}_{4}$, applied at different temperatures was analyzed. The $\mathrm{B}_{4} \mathrm{C}$ coating was applied to the substrate at $1000^{\circ} \mathrm{C}$. Then the substrate and the $\mathrm{B}_{4} \mathrm{C}$ coating were heated to $1500^{\circ} \mathrm{C}$. At that temperature the $\mathrm{Si}_{3} \mathrm{~N}_{4}$ coating was added. The temperature was then lowered to $20^{\circ} \mathrm{C}$ and the stresses in the substrate and coatings were calculated. This process was illustrated in Fig. 2.1. Results for this infinitely wide case were shown in Table 6.1 on page 92 and a comparison of results using temperature-dependent properties and temperature-independent properties was shown in Fig. 6.5. Realistic dimensions for a finite width composite were shown in Fig. 6.2b on page 90. These dimensions are $w_{s u b}=8 \mathrm{~mm}, t_{s u b}=1 \mathrm{~mm}$, and $r=0.5 \mathrm{~mm}$. The coating thicknesses are $t_{1}=0.1 \mathrm{~mm}$ for the $\mathrm{B}_{4} \mathrm{C}$ coating and $t_{2}=0.2 \mathrm{~mm}$ for the $\mathrm{Si}_{3} \mathrm{~N}_{4}$ coating.

The room temperature stresses $\left(20^{\circ} \mathrm{C}\right)$ in both coatings and the substrate were calculated as a function of $s$ for the realistic finite width geometry at different thickness locations within the coatings and the substrate. These locations are indicated in Fig. 6.41. The coordinate $d$ is normalized by the total coating thickness $(0.3 \mathrm{~mm})$. The $d$-coordinate is zero 


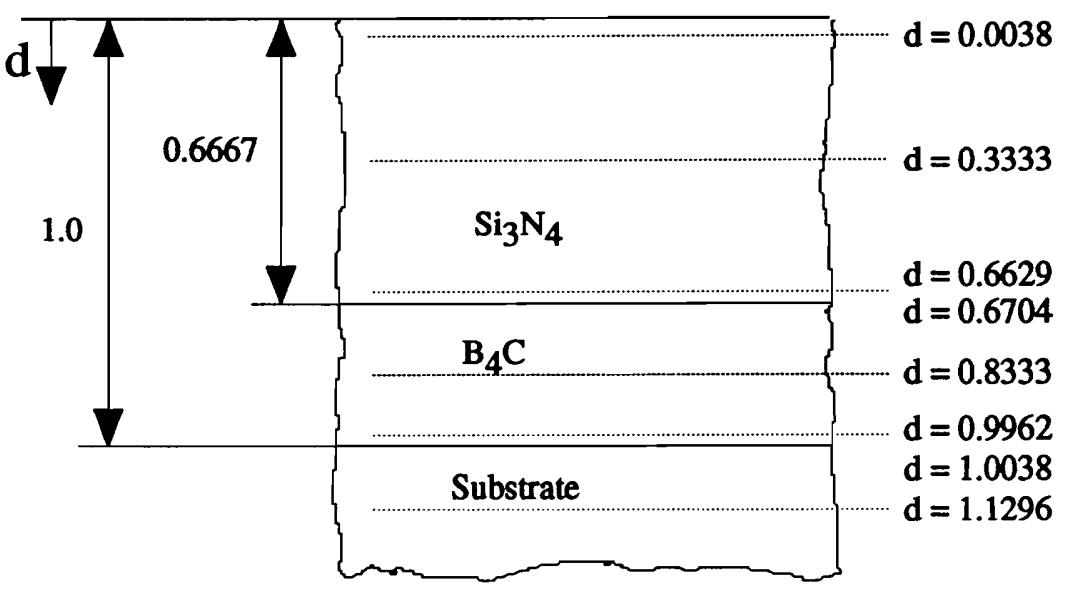

Figure 6.41: Normalized thickness locations for stress calculations.

at the free surface and unity at the interface between the $\mathrm{B}_{\mathbf{4}} \mathrm{C}$ coating and the substrate. The $\mathrm{B}_{4} \mathrm{C}-\mathrm{Si}_{3} \mathrm{~N}_{4}$ interface is at $d=0.6667$. Unless otherwise noted, all stresses discussed in this section were normalized by the maximum far field stress in the $\mathrm{B}_{4} \mathrm{C}$ coating, which is shown in Table 6.1. This stress was chosen for the normalization because it is the largest of all stress components, and therefore it is critical to reduce this stress to avoid failure of the coatings. Also, later in this section, stresses as a function of $d$ are discussed. If stresses were normalized by the far field stresses in the individual coatings and the substrate, the stress components $\bar{\sigma}_{n}$ and $\bar{\tau}_{s n}$, which are continuous across material interfaces, would appear to be discontinuous. Normalization of the stresses in the coatings and the substrate by the same maximum far field stress avoids this problem.

Stresses in the $\mathrm{Si}_{3} \mathrm{~N}_{4}$ coating are shown in Fig. 6.42 near the free surface $(d=0.0038)$, at the center of the coating $(d=0.3333)$, and near the $\mathrm{Si}_{3} \mathrm{~N}_{4}-\mathrm{B}_{4} \mathrm{C}$ interface at $d=0.6629$. Similarly, stresses in the $\mathrm{B}_{4} \mathrm{C}$ coating are shown in Fig. 6.43 near the $\mathrm{Si}_{3} \mathrm{~N}_{4}-\mathrm{B}_{4} \mathrm{C}$ interface at $d=0.6704$, at the center of the coating $(d=0.8333)$, and near the $\mathrm{B}_{4} \mathrm{C}$-substrate interface at $d=0.9962$. The stresses in the substrate are shown in Fig. 6.44 near the $\mathrm{B}_{4} \mathrm{C}$-substrate interface at $d=1.0038$, and at two other locations further into the substrate, $d=1.0667$, 
Table 6.9: Comparison of far field stresses calculated using the ISPSA and the finite element model (FEM) for multiple coatings.

\begin{tabular}{|c|c|c|c|c|}
\hline & Case & Substrate & $\mathrm{B}_{4} \mathrm{C}$ & $\mathrm{Si}_{3} \mathrm{~N}_{4}$ \\
\hline \multirow{2}{*}{$\bar{\sigma}_{s}$} & FEM & -0.1734 & 1.0029 & 0.3830 \\
& ISPSA & -0.1745 & 1.0000 & 0.3724 \\
\hline \multirow{2}{*}{$\bar{\sigma}_{z}$} & FEM & -0.1798 & 0.9848 & 0.3485 \\
& ISPSA & -0.1745 & 1.0000 & 0.3724 \\
\hline
\end{tabular}

and $d=1.1296$. As was mentioned previously, through-the-thickness gradients in the stress components are proportional to the distance between the lines for the different thickness locations, and gradients in the tangential direction (s-direction) are proportional to the slope of the curves in the graphs.

It can be seen in Figs. 6.42 - 6.44 that away from the corner, for both coatings and the substrate, almost no gradients are present in the tangential stress $\bar{\sigma}_{s}$ and the axial stress $\bar{\sigma}_{z}$, in either the through-the-thickness or the tangential directions. Also, away from the corner the normal stress $\bar{\sigma}_{n}$ and the shear stress $\bar{\tau}_{s n}$ were zero. Thus, stresses in this region should be predicted accurately by a simpler analysis, e.g., the ISPSA. That this indeed is the case is shown in Table 6.9. In Table 6.9 the normalized far-field stresses calculated using the ISPSA are compared to the normalized far-field stresses calculated using the finite element model, denoted as FEM in the table. As can be seen, the stresses are in excellent agreement. Because the moduli of the substrate were smaller than the moduli of the coatings, and because the substrate had a much larger volume than the coatings, the relative magnitude of the tangential and axial stresses in the substrate was small compared to the coatings. Because in the far field the coefficient of thermal expansion of the substrate in the s-direction was smaller than the coefficient of thermal expansion of either coating, the tangential and the axial stresses in the substrate were compressive and the tangential and axial stresses in the coatings were tensile.

In the corner region, the tangential stress $\bar{\sigma}_{s}$ in the substrate (Fig. 6.44) varied in the 


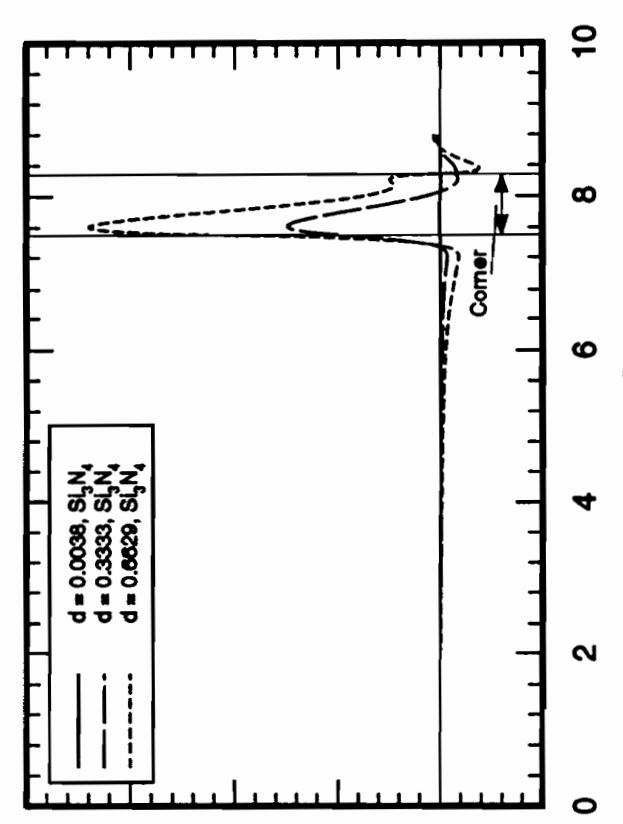

꿍 당 웅 융
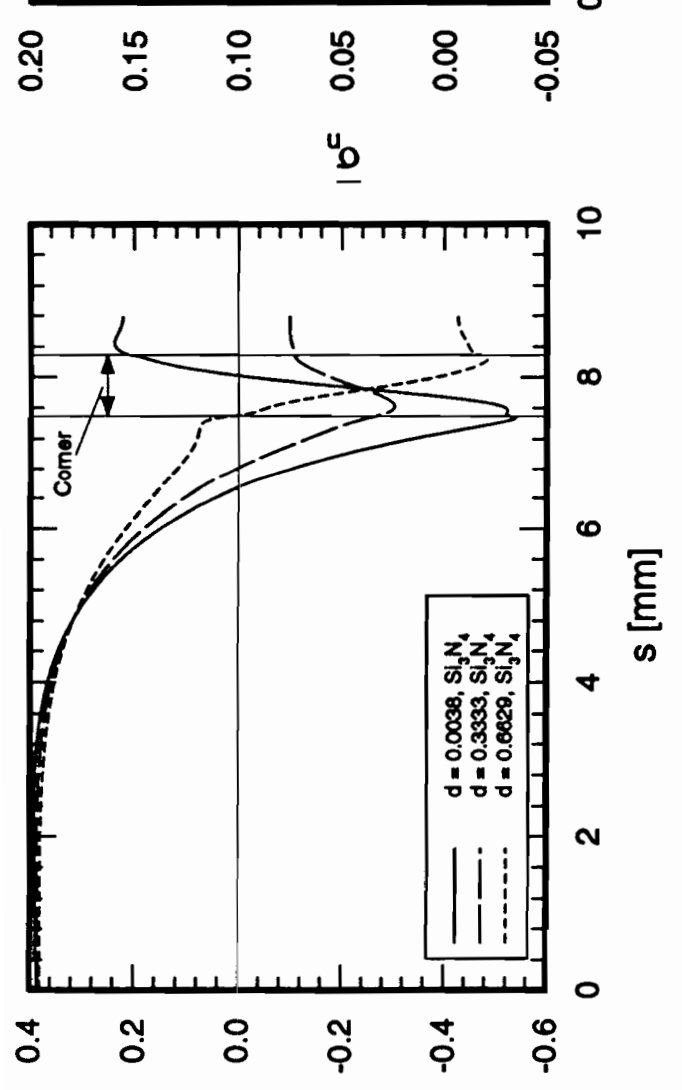

so

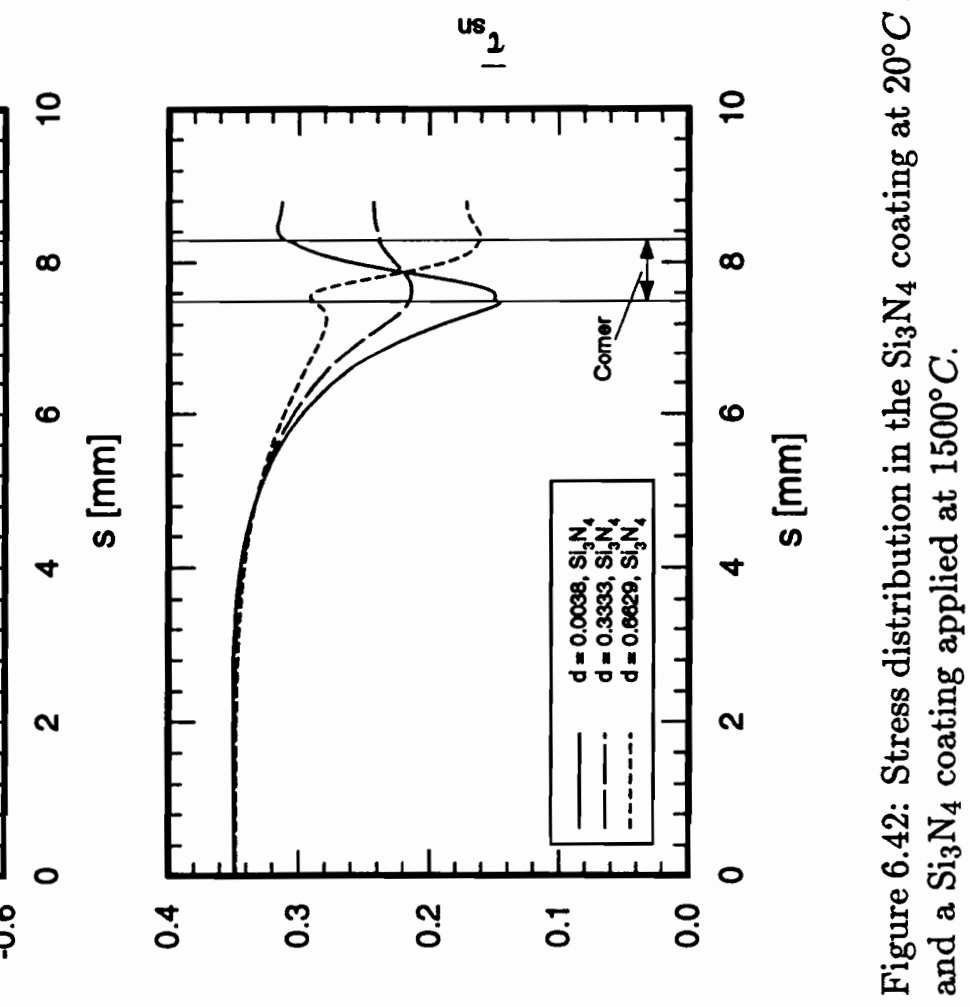



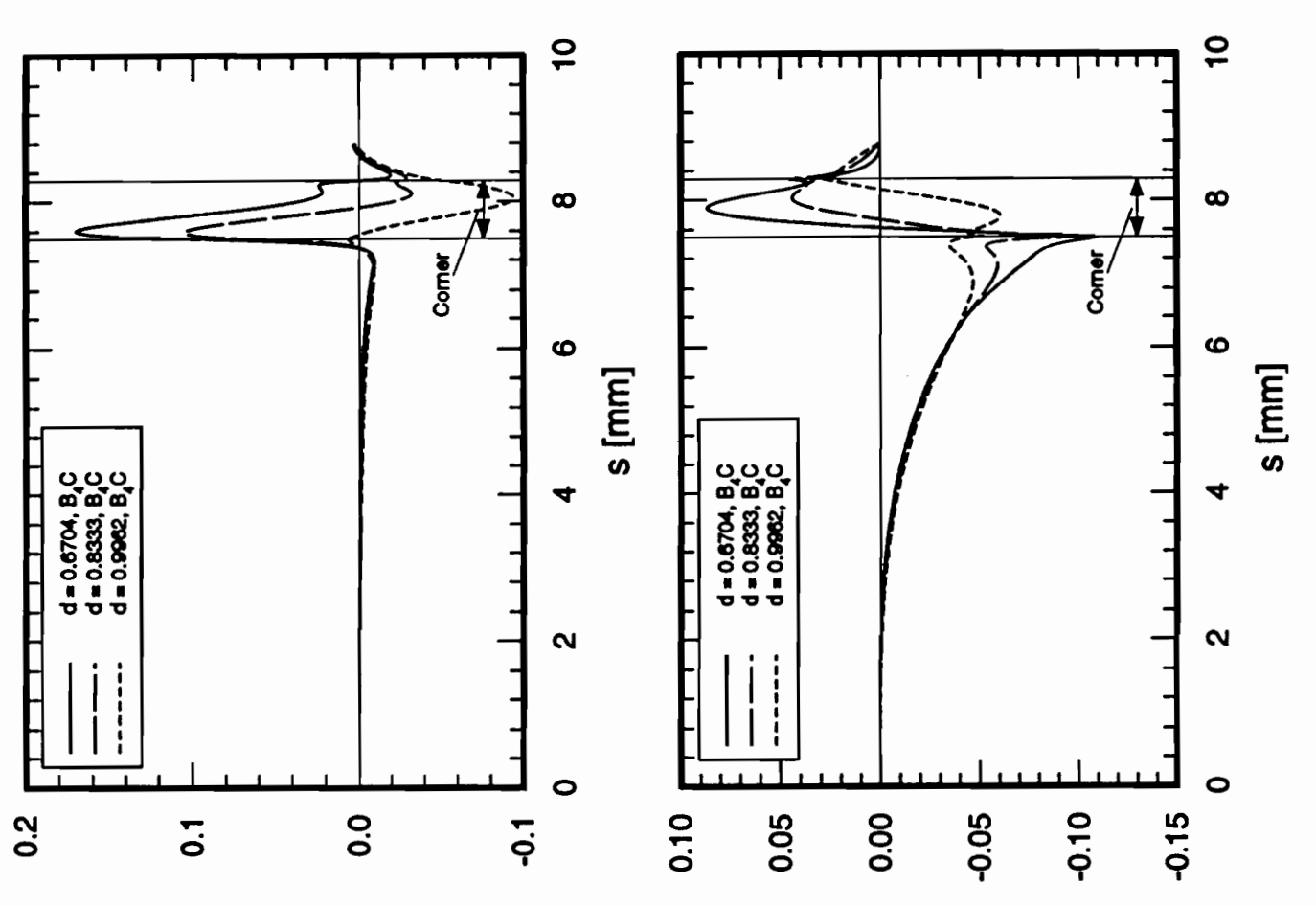

○

है

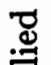
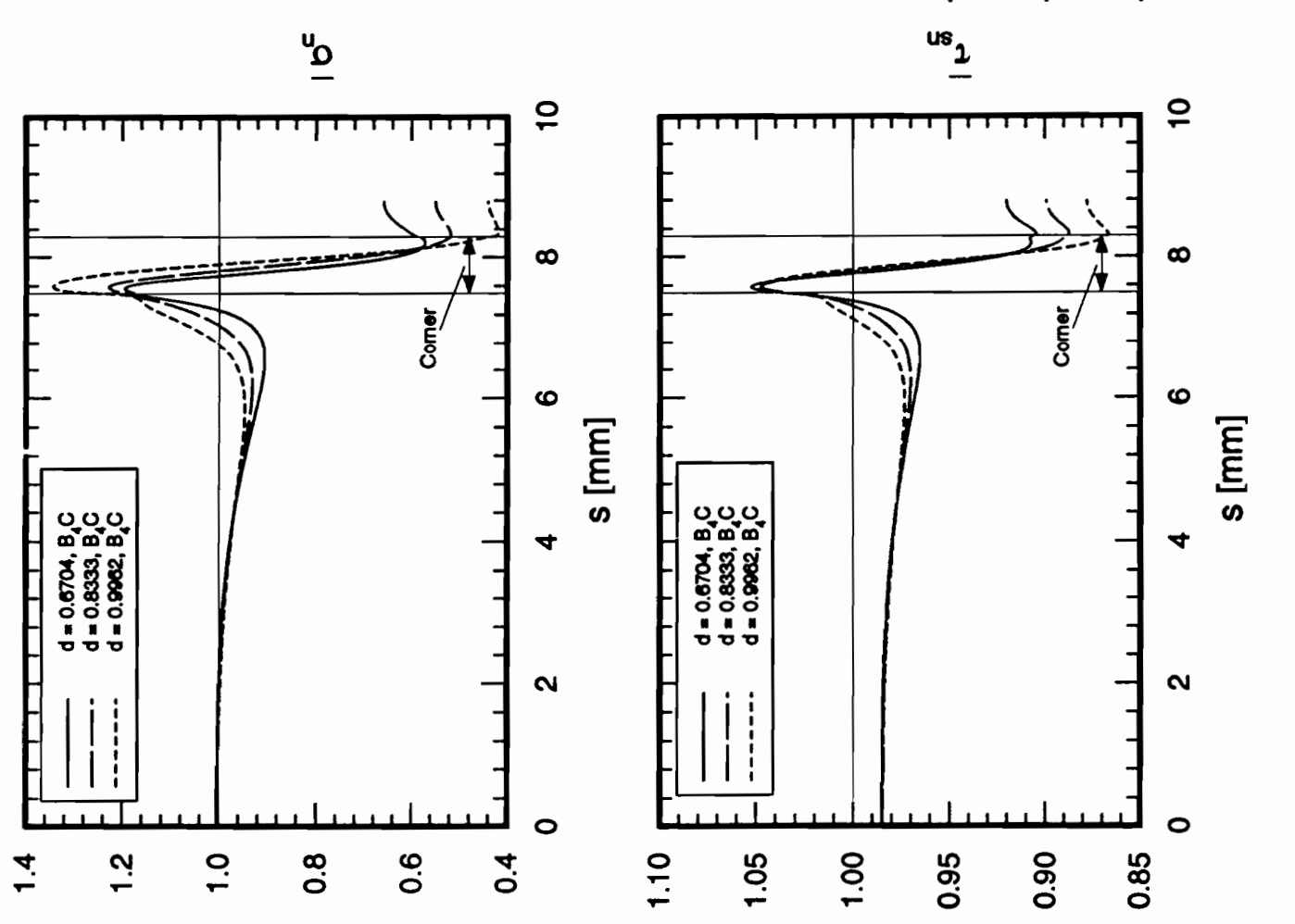

$\underline{\text { so }}$

zo 

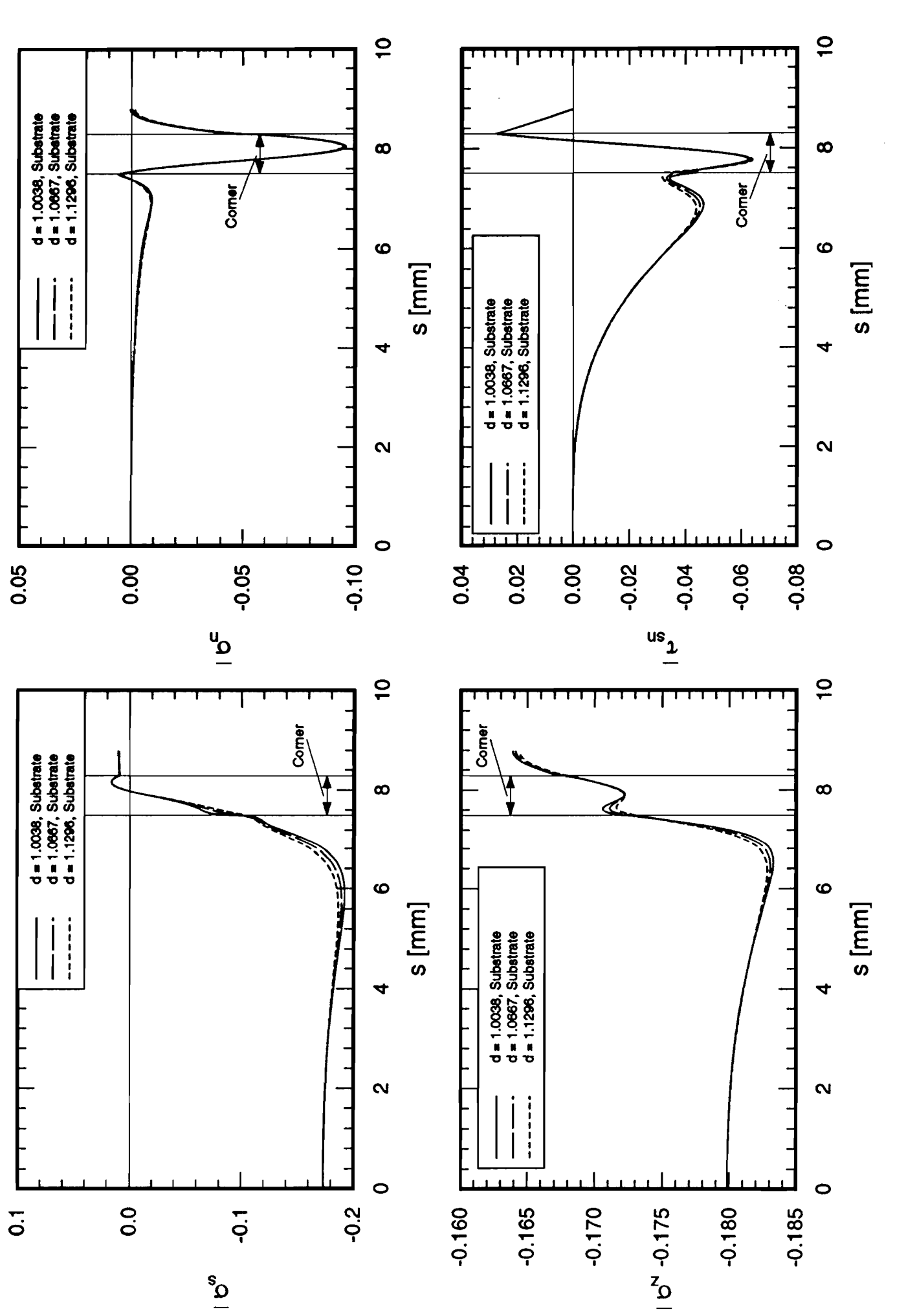

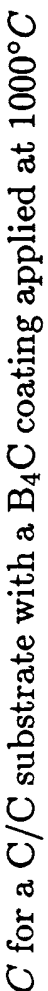

ㅇำ

藏

荬

范

U

영

$\exists \stackrel{9}{4}$

若

总

总.

至㖞

迷望

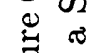

雳 
tangential direction, while there was almost no variation through the thickness. In contrast, for both coatings a large variation of the tangential stress in the corner region can be observed in the tangential direction and through-the-thickness of the coatings. A large tensile stress concentration was present in the $\mathrm{B}_{4} \mathrm{C}$ coating (Fig. 6.43) at the beginning of the corner. This stress concentration was largest near the $\mathrm{B}_{4} \mathrm{C}$ substrate interface. When moving through the corner along the $s$-coordinate, after the stress concentration the stress decreased rapidly. This decrease was caused by the orthotropy of the substrate and the rotation of the s-n-z-coordinate system in the corner region, as was explained in Section 5.4. Before the corner, the $s$-coordinate was parallel to the stiff, low coefficient of thermal expansion direction of the substrate, which caused large tangential stresses. On the other hand, after the corner, the s-coordinate was parallel to the soft, high coefficient of thermal expansion direction of the substrate, which resulted in much lower tangential stresses in the coating. The tangential stress in the $\mathrm{B}_{4} \mathrm{C}$ coating after the corner reached only about $40 \%$ of the magnitude of this stress component in the far field. Due to the large stress concentration at the beginning of the corner at the $\mathrm{B}_{4} \mathrm{C}$-substrate interface, tensile failure in the coatings would most likely initiate at that location.

As can be seen in Fig. 6.42, the change in the tangential stress $\bar{\sigma}_{s}$ in the $\mathrm{Si}_{3} \mathrm{~N}_{4}$ coating as a function of $s$ when traversing the corner was even more dramatic than for the $\mathrm{B}_{4} \mathrm{C}$ coating, though the magnitude of the tangential stress was much lower. Near the free surface $(d=0)$, the tangential stress changed from a tensile stress in the far field, to a compressive stress that is larger in magnitude at the begiuning of the corner, back to a tensile stress after the corner. In contrast to this, when moving along the $s$-coordinate through the corner, near the $\mathrm{Si}_{3} \mathrm{~N}_{4}-\mathrm{B}_{4} \mathrm{C}$ interface $(d=0.6629)$ the tangential stress gradually decreased from its tensile far field value to a larger compressive stress. Therefore, near the horizontal line of symmetry, a very large through-the-thickness gradient of the tangential stress in the $\mathrm{Si}_{3} \mathrm{~N}_{4}$ coating was present.

For both coatings and the substrate, the axial stress $\bar{\sigma}_{z}$ varied in a manner similar to the tangential stress $\bar{\sigma}_{s}$ variation. However, the change of this stress in the corner region 
was much smaller. In the $\mathrm{Si}_{3} \mathrm{~N}_{4}$ coating the axial stress remained tensile throughout.

The normal stress $\bar{\sigma}_{n}$ was confined to the corner region for both coatings and the substrate. In both coatings, in the corner region, large tangential and through-the-thickness gradients were present in the normal stress. Though there was a large tangential gradient in the normal stress for the substrate, the normal stress did not vary through-the-thickness of the substrate. In Fig. 6.42 for the $\mathrm{Si}_{3} \mathrm{~N}_{4}$ coating, it can be seen that the normal stress satisfied the zero-stress condition at the free surface. When moving away from the free surface, into the coating, the normal stress increased rapidly in the corner region and reached its largest tensile value near the $\mathrm{Si}_{3} \mathrm{~N}_{4}-\mathrm{B}_{4} \mathrm{C}$ interface at $d=0.6629$. This indicates that the $\mathrm{Si}_{3} \mathrm{~N}_{4}$ and $\mathrm{B}_{4} \mathrm{C}$ coatings may delaminate in the corner region. The normal stress in the $\mathrm{B}_{4} \mathrm{C}$ coating (Fig. 6.43) near the $\mathrm{Si}_{3} \mathrm{~N}_{4}-\mathrm{B}_{4} \mathrm{C}$ interface had the same magnitude and distribution as the normal stress had in the $\mathrm{Si}_{3} \mathrm{~N}_{4}$ coating near that interface. This indicates that the continuity of the normal stress across the interface is satisfied. Inside the $\mathrm{B}_{\mathbf{4}} \mathrm{C}$ coating, the normal stress decreased rapidly and was compressive near the $\mathrm{B}_{4} \mathrm{C}$-substrate interface. $\mathrm{A}$ comparison of Figs. 6.43 and 6.44 shows that the continuity of the normal stress was also satisfied at the $\mathrm{B}_{4} \mathrm{C}$-substrate interface.

As with the normal stress, the shear stress $\bar{\tau}_{s n}$ was most predominant in the corner. However, the shear stress decreased only slowly when moving away from the corner and reached zero about 8-10 radii away from the corner. This fact that the shear stress has a larger reaching influence than the normal stress has been discussed and observed before. In both coatings and the substrate, the shear stress was negative at the beginning of the corner and positive after the corner. The largest shear stress occurred at the $\mathrm{Si}_{3} \mathrm{~N}_{4}-\mathrm{B}_{4} \mathrm{C}$ interface. An inspection of the shear stress in both coatings and the substrate shows that the shear stress satisfied the zero-stress condition at the free surface and the continuity condition at the interfaces.

As was shown in the above discussion, a large stress concentration was present in the tangential stress in the $\mathrm{B}_{4} \mathrm{C}$ coating near the corner. Also, fairly large normal and shear stresses were present in the corner region. In the previous sections it was shown that stress 
concentrations can be significantly reduced if gradient coatings are used in which properties vary from a soft material near the coating-substrate interface to the original coating material some distance away from that interface. In the following section it is investigated if for the case for multiple coating layers, the stress concentration in the tangential stress component can be reduced by applying a gradient coating as part of the coating.

\subsubsection{Gradient Coatings Applied at Multiple Temperatures}

To investigate whether gradient coatings can be used to reduce stresses for multiple coatings on a $\mathrm{C} / \mathrm{C}$ substrate, the discrete $\mathrm{B}_{4} \mathrm{C}$ and $\mathrm{Si}_{3} \mathrm{~N}_{4}$ coatings were partially replaced by a gradient coating in which the properties varied from pyrolytic carbon near the $\mathrm{C} / \mathrm{C}$ substrate to $\mathrm{B}_{4} \mathrm{C}$, and then to $\mathrm{Si}_{3} \mathrm{~N}_{4}$. For analysis purposes this gradient coating was split into two distinct linear gradient coatings. In the inner gradient coating, properties varied linearly from pyrolytic carbon to $\mathrm{B}_{\mathbf{4}} \mathrm{C}$, and in the outer gradient coating, properties varied linearly from $\mathrm{B}_{4} \mathrm{C}$ to $\mathrm{Si}_{3} \mathrm{~N}_{4}$. The outmost coating was pure $\mathrm{Si}_{3} \mathrm{~N}_{4}$. Each of the two linear gradient coatings and the discrete $\mathrm{Si}_{3} \mathrm{~N}_{4}$ coating had the same thickness (1/3 of the total thickness). This coating geometry is shown in Fig. 6.45a.

The application temperature of the three coatings was also studied. As shown in Appendix $A$, the normal application temperature for the pyrolytic carbon and the $\mathrm{B}_{4} \mathrm{C}$ is $1000^{\circ} \mathrm{C}$, while the normal application temperature for the $\mathrm{Si}_{3} \mathrm{~N}_{4}$ coating is $1500^{\circ} \mathrm{C}$. This leaves the question as to which temperature the $\mathrm{B}_{4} \mathrm{C}-\mathrm{Si}_{3} \mathrm{~N}_{4}$ gradient coating should be applied. Four different temperature scenarios were studied. For all scenarios, the pyrolytic carbon- $\mathrm{B}_{4} \mathrm{C}$ gradient coating was applied at $1000^{\circ} \mathrm{C}$ and the $\mathrm{Si}_{3} \mathrm{~N}_{4}$ coating was applied at $1500^{\circ} \mathrm{C}$. The four cases for the $\mathrm{B}_{4} \mathrm{C}-\mathrm{Si}_{3} \mathrm{~N}_{4}$ gradient coating were:

1. Application of the entire $\mathrm{B}_{4} \mathrm{C}-\mathrm{Si}_{3} \mathrm{~N}_{4}$ gradient coating at $1000^{\circ} \mathrm{C}$, i.e., at the application temperature of a $\mathrm{B}_{4} \mathrm{C}$ coating.

2. Application of the entire $\mathrm{B}_{4} \mathrm{C}-\mathrm{Si}_{3} \mathrm{~N}_{4}$ gradient coating at $1250^{\circ} \mathrm{C}$, i.e., at the midpoint between the application temperatures for $\mathrm{B}_{4} \mathrm{C}$ and $\mathrm{Si}_{3} \mathrm{~N}_{4}$ coatings. 


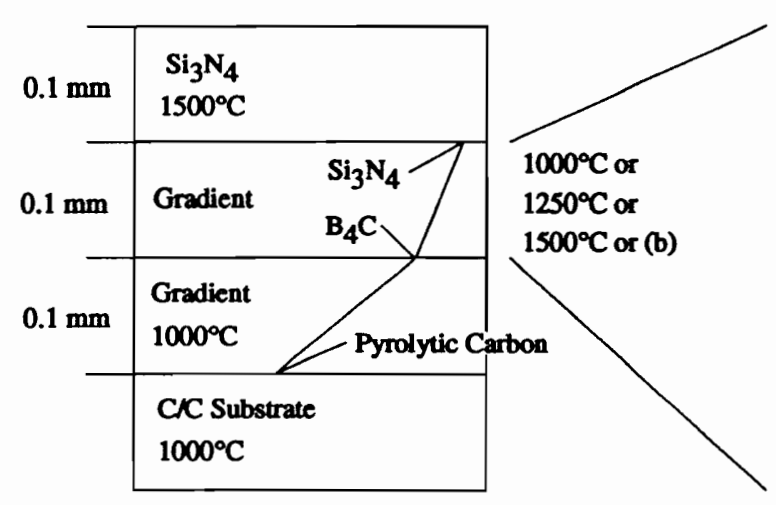

(a)

\begin{tabular}{|c|r|}
\hline $1475^{\circ} \mathrm{C}$ & $100 \% \mathrm{Si}_{3} \mathrm{~N}_{4}$ \\
\hline $1425^{\circ} \mathrm{C}$ & $10 \% \mathrm{~B}_{4} \mathrm{C}, 90 \% \mathrm{Si}_{3} \mathrm{~N}_{4}$ \\
\hline $1375^{\circ} \mathrm{C}$ & $20 \% \mathrm{~B}_{4} \mathrm{C}, 80 \% \mathrm{Si}_{3} \mathrm{~N}_{4}$ \\
\hline $1325^{\circ} \mathrm{C}$ & $30 \% \mathrm{~B}_{4} \mathrm{C}, 70 \% \mathrm{Si}_{3} \mathrm{~N}_{4}$ \\
\hline $1275^{\circ} \mathrm{C}$ & $40 \% \mathrm{~B}_{4} \mathrm{C}, 60 \% \mathrm{Si}_{3} \mathrm{~N}_{4}$ \\
\hline $1225^{\circ} \mathrm{C}$ & $50 \% \mathrm{~B}_{4} \mathrm{C}, 50 \% \mathrm{Si}_{3} \mathrm{~N}_{4}$ \\
\hline $1175^{\circ} \mathrm{C}$ & $60 \% \mathrm{~B}_{4} \mathrm{C}, 40 \% \mathrm{Si}_{3} \mathrm{~N}_{4}$ \\
\hline $1125^{\circ} \mathrm{C}$ & $70 \% \mathrm{~B}_{4} \mathrm{C}, 30 \% \mathrm{Si}_{3} \mathrm{~N}_{4}$ \\
\hline $1075^{\circ} \mathrm{C}$ & $80 \% \mathrm{~B}_{4} \mathrm{C}, 20 \% \mathrm{Si}_{3} \mathrm{~N}_{4}$ \\
\hline $1025^{\circ} \mathrm{C}$ & $90 \% \mathrm{~B}_{4} \mathrm{C}, 10 \% \mathrm{Si}_{3} \mathrm{~N}_{4}$ \\
\hline & $100 \% \mathrm{~B}_{4} \mathrm{C}$
\end{tabular}

(b)

Figure 6.45: Geometry of coatings for multiple gradient coatings.

3. Application of the entire $\mathrm{B}_{4} \mathrm{C}-\mathrm{Si}_{3} \mathrm{~N}_{4}$ gradient coating at $1500^{\circ} \mathrm{C}$, i.e., at the application temperature for pure $\mathrm{Si}_{3} \mathrm{~N}_{4}$ coating.

4. Continuous variation of the application temperature from $1000^{\circ} \mathrm{C}$ to $1500^{\circ} \mathrm{C}$, as the $\mathrm{B}_{4} \mathrm{C}-\mathrm{Si}_{3} \mathrm{~N}_{4}$ gradient coating thickness increases, and hence the material composition changes. This was approximated by dividing the $\mathrm{B}_{4} \mathrm{C}-\mathrm{Si}_{3} \mathrm{~N}_{4}$ gradient coating into 10 smaller gradient coatings, each of which was applied at progressively increasing temperatures. This is illustrated in Fig. $6.45 \mathrm{~b}$, in which the properties at the interfaces between the smaller gradient coatings and the application temperatures of these coatings are indicated.

Because one of the main goals for using gradient coatings was to reduce the stress concentrations in the tangential stress in the corner region, maximum tangential stresses in the coatings and the substrate were compared at $20^{\circ} \mathrm{C}$. These stresses are shown in Table 6.10 in the substrate, the pyrolytic carbon- $\mathrm{B}_{4} \mathrm{C}$ gradient coating, the $\mathrm{B}_{4} \mathrm{C}-\mathrm{Si}_{3} \mathrm{~N}_{4}$ gradient coating, and the $\mathrm{Si}_{3} \mathrm{~N}_{4}$ coating for the different application temperature scenarios. As a reference, the maximum tangential stresses in the $\mathrm{B}_{4} \mathrm{C}$ coating and the $\mathrm{Si}_{3} \mathrm{~N}_{4}$ coating for the discrete coating case are also shown. These stresses are taken from the last section, Figs. 6.42 - 6.44. The locations at which the stresses occurred are indicated in the table. As can be seen in 
Table 6.10: Maximum tangential stresses $\bar{\sigma}_{s}$ for multiple coatings on a $\mathrm{C} / \mathrm{C}$ substrate

\begin{tabular}{|c|c|c|c|c|c|c|}
\hline \multirow{2}{*}{ Material } & \multicolumn{4}{|c|}{$\mathrm{B}_{4} \mathrm{C}-\mathrm{Si}_{3} \mathrm{~N}_{4}$ gradient coating applied at: } & \multirow{2}{*}{ Material } & \multirow{2}{*}{ Discrete } \\
\hline & $1000^{\circ} \mathrm{C}$ & $1250^{\circ} \mathrm{C}$ & $1500^{\circ} \mathrm{C}$ & Continuous & & \\
\hline $\mathrm{Si}_{3} \mathrm{~N}_{4}$ & $-0.5100^{2}$ & $-0.5105^{a}$ & $-0.5115^{a}$ & $-0.5121^{\mathrm{a}}$ & $\mathrm{Si}_{3} \mathrm{~N}_{4}$ & $-0.5398^{a}$ \\
\hline $\mathrm{B}_{4} \mathrm{C}-\mathrm{Si}_{3} \mathrm{~N}_{4}$ gradient & $1.0492^{b}$ & $1.3585^{b}$ & $1.6391^{b}$ & $1.0531^{b}$ & $\mathrm{~B}, \mathrm{C}$ & $13498^{a}$ \\
\hline Carbon- $\mathrm{B}_{4} \mathrm{C}$ gradient & $1.0387^{\mathrm{a}}$ & $1.0230^{\mathrm{a}}$ & $1.0851^{\mathrm{a}}$ & $1.0343^{\mathrm{a}}$ & & \\
\hline Substrate & $-0.1800^{c}$ & $-0.1972^{c}$ & $-0.2113^{c}$ & $-0.1906^{c}$ & Substrate & $-0.1920^{c}$ \\
\hline
\end{tabular}

${ }^{a}$ occurred in corner near interface between $\mathrm{Si}_{3} \mathrm{~N}_{4}$ and $\mathrm{B}_{4} \mathrm{C}-\mathrm{Si}_{3} \mathrm{~N}_{4}$ gradient coating

boccurred in far field

c occurred near the corner near the substrate-coating interface

Table 6.10, the maximum tangential stress in the substrate and in the $\mathrm{Si}_{3} \mathrm{~N}_{4}$ coating did not significantly change for the different cases, and when compared to the discrete coating case. Also, for the gradient coating cases, the stresses in the pyrolytic carbon- $\mathrm{B}_{4} \mathrm{C}$ gradient coating did not change significantly. However, the maximum tangential stress in the $\mathrm{B}_{4} \mathrm{C}$ $\mathrm{Si}_{3} \mathrm{~N}_{4}$ gradient coating strongly depended on the application temperature of that coating. For application of the $\mathrm{B}_{4} \mathrm{C}-\mathrm{Si}_{3} \mathrm{~N}_{4}$ gradient coating at discrete temperatures, the maximum stress increased with increasing application temperature. As indicated in the table, for all cases the maximum tangential stress in the $\mathrm{B}_{4} \mathrm{C}-\mathrm{Si}_{3} \mathrm{~N}_{4}$ gradient coating occurred in the far field. The maximum tangential stress for the discrete coating case occurred in the corner region at the interface between the $\mathrm{B}_{4} \mathrm{C}$ coating and the substrate. In the gradient coating cases the maximum tangential stress occured at the interface between the $\mathrm{B}_{4} \mathrm{C}-\mathrm{Si}_{3} \mathrm{~N}_{4}$ gradient coating and the pyrolytic carbon- $\mathrm{B}_{4} \mathrm{C}$ gradient coating. At this interfóce the material in the gradient coating was $100 \% \mathrm{~B}_{4} \mathrm{C}$. When the coating was applied at $1000^{\circ} \mathrm{C}$, compared to the discrete coating case, the maximum tangential stress in the $\mathrm{B}_{4} \mathrm{C}-\mathrm{Si}_{3} \mathrm{~N}_{4}$ gradient coating was significantly reduced and its location was shifted away from the corner to the far field. This was caused by a reduction of the stress concentration in the corner. In contrast, when the coating was applied at $1500^{\circ} \mathrm{C}$, the maximum tangential stress in the $\mathrm{B}_{4} \mathrm{C}-\mathrm{Si}_{3} \mathrm{~N}_{4}$ gradient coating was larger than the maximum tangential stress for the discrete coating case, although it still occurred in the far field, away from the corner. This fact indicates 
that when the $\mathrm{B}_{4} \mathrm{C}-\mathrm{Si}_{3} \mathrm{~N}_{4}$ gradient coating was applied at higher temperatures, the far field stress increased compared to the discrete coating case and compared to the case for which that gradient coating was applied at $1000^{\circ} \mathrm{C}$. The fact that the far field stress increased for increasing application temperature of the $\mathrm{B}_{4} \mathrm{C}-\mathrm{Si}_{3} \mathrm{~N}_{4}$ gradient coating can be explained by considering an infinitely wide $\mathrm{C} / \mathrm{C}$ substrate with discrete $\mathrm{B}_{4} \mathrm{C}$ and $\mathrm{Si}_{3} \mathrm{~N}_{4}$ coatings, which can be analyzed using the ISPSA. In Table 6.11 stresses from several ISPSA analyses in which the $\mathrm{B}_{4} \mathrm{C}$ coating was applied at different temperatures are shown at $1500^{\circ} \mathrm{C}$, the temperature at which the $\mathrm{Si}_{3} \mathrm{~N}_{4}$ coating was applied, and at $20^{\circ} \mathrm{C}$. The stresses for $1500^{\circ} \mathrm{C}$ were normalized by the stress at $1500^{\circ} \mathrm{C}$ for the $\mathrm{B}_{4} \mathrm{C}$ coating in Table 6.1. Similarly, the stresses at $20^{\circ} \mathrm{C}$ were normalized by the stress for $20^{\circ} \mathrm{C}$ for the $\mathrm{B}_{4} \mathrm{C}$ coating in Table 6.1. As can be seen in Table 6.11, when the $\mathrm{B}_{4} \mathrm{C}$ coating was applied at higher temperatures, the residual tangential stress in the $\mathrm{B}_{4} \mathrm{C}$ coating was lower at the application temperature of the $\mathrm{Si}_{3} \mathrm{~N}_{4}$ coating. This residual stress in the $\mathrm{B}_{4} \mathrm{C}$ coating was compressive at the application temperature of the $\mathrm{Si}_{3} \mathrm{~N}_{4}$ coating. However, tensile stresses are generated in the $\mathrm{B}_{4} \mathrm{C}$ coating as the composite is cooled from $1500^{\circ} \mathrm{C}$ to room temperature, after the $\mathrm{Si}_{3} \mathrm{~N}_{4}$ coating was applied, because the $\mathrm{B}_{4} \mathrm{C}$ coating had a larger coefficient of thermal expansion than the $\mathrm{Si}_{3} \mathrm{~N}_{4}$ coating, or the substrate. Therefore, the lower the compressive stress was in the $\mathrm{B}_{4} \mathrm{C}$ coating for $1500^{\circ} \mathrm{C}$, the higher the tensile stress was in this coating at room temperature.

It is interesting to compare the through-the-thickness stress distributions for the different coating cases. For this comparison room temperature stresses were calculated as a funciion of the normalized thickness coordinate $d$ at different locations along the circumference of the composite. Recall from Fig. 6.41, the coordinate $d$ is the thickness coordinate normalized by the total coating thickness. The coordinate $d$ is zero at the free surface and increases when moving into the coatings. At the pyrolytic carbon-substrate interface $d=1$. The $s$ locations at which the stresses are shown as a function of $d$ are indicated in Fig. 6.46. The through-the-thickness variation of the stresses at the locations indicated in Fig. 6.46 are shown in Figs. $6.47-6.51$. For locations 1 and 5, only the tangential stress $\bar{\sigma}_{s}$ and the axial 
Table 6.11: Normalized stresses ${ }^{1}$ in a $\mathrm{B}_{\mathbf{4}} \mathrm{C}$ coating for different coating application temperatures.

\begin{tabular}{|c|c|c|}
\hline Application Temp. & \multicolumn{2}{|c|}{ Stresses in $\mathrm{B}_{4} \mathrm{C}$ coating at } \\
\cline { 2 - 3 } of $\mathrm{B}_{4} \mathrm{C}$ coating & $1500^{\circ} \mathrm{C}$ & $20^{\circ} \mathrm{C}$ \\
\hline $1000^{\circ} \mathrm{C}$ & $1^{\mathrm{c}}$ & $1^{\mathrm{t}}$ \\
$1250^{\circ} \mathrm{C}$ & $0.4722^{\mathrm{c}}$ & $1.3064^{\mathrm{t}}$ \\
$1500^{\circ} \mathrm{C}$ & $0^{\mathrm{c}}$ & $1.5806^{\mathrm{t}}$ \\
\hline
\end{tabular}

${ }^{1}$ Stresses are normalized by the stresses given for the $\mathrm{B}_{\mathbf{4}} \mathrm{C}$ coating in Table 6.1 .

${ }^{t}$ tensile stress

${ }^{c}$ compressive stress

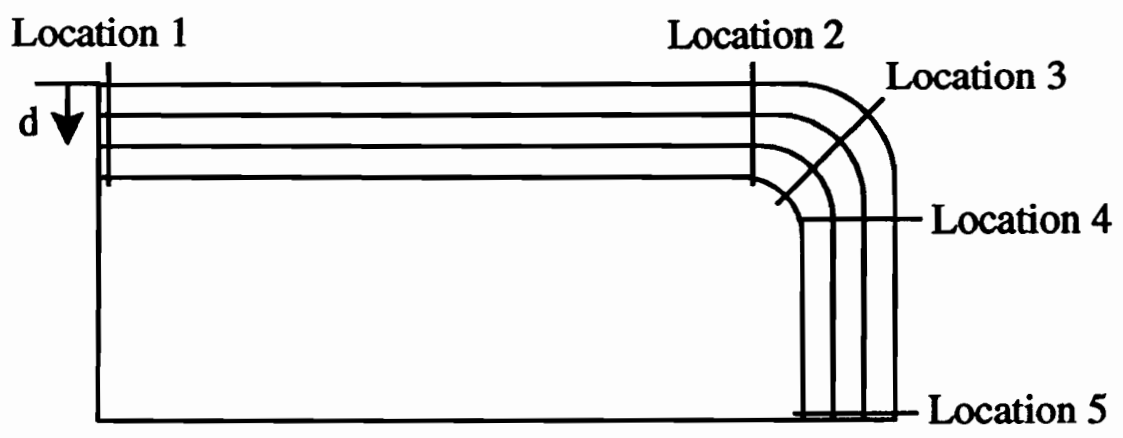

Figure 6.46: Locations for through-thickness plots.

stress $\bar{\sigma}_{z}$ are shown, because the normal stress $\bar{\sigma}_{n}$ and the shear stress $\bar{\tau}_{s n}$ are zero at those locations.

First consider the stress distributions through-the-thickness in the far field, location 1 , shown in Fig. 6.47. For the discrete coating case, the tangential stress $\bar{\sigma}_{s}$ and the axial stress $\bar{\sigma}_{z}$ were constant through the thickness of each coating and in the substrate. For the gradient coating cases, the stresses in the substrate and in the $\mathrm{Si}_{3} \mathrm{~N}_{4}$ coating were close to the stresses for the discrete coating case. In the pyrolytic carbon- $\mathrm{B}_{4} \mathrm{C}$ gradient coating, the axial and tangential stress were close for all gradient coating cases. The stresses decreased from 
their largest value at the interface between the two gradient coatings towards their lowest value at the pyrolytic carbon-substrate interface. This decrease in stress was caused by the decrease in modulus when moving from the interface of the two gradient coatings towards the pyrolytic carbon-substrate interface. In contrast, the distribution of the stresses in the $\mathrm{B}_{\mathbf{4}} \mathrm{C}$ $\mathrm{Si}_{3} \mathrm{~N}_{4}$ gradient coating was quite different for the various cases. As described earlier, the maximum stress in this coating at the interface between the two gradient coatings increased significantly with increasing application temperature of the coating. Since the moduli of the $\mathrm{Si}_{3} \mathrm{~N}_{4}$ and the $\mathrm{B}_{4} \mathrm{C}$ were very close, the increase in the stresses with increasing $d$ in this coating was caused by an increasing coefficient of thermal expansion, as the material gradually changes from $\mathrm{Si}_{3} \mathrm{~N}_{4}$ to $\mathrm{B}_{4} \mathrm{C}$. While the stresses for the application of the gradient coating at discrete temperatures were discontinuous either at the interface between the two gradient coatings, or at the interface between the $\mathrm{Si}_{3} \mathrm{~N}_{4}$ coating and the $\mathrm{B}_{4} \mathrm{C}-\mathrm{Si}_{3} \mathrm{~N}_{4}$ gradient coating, the stresses were continuous across both interfaces for the case in which the $\mathrm{B}_{4} \mathrm{C}-\mathrm{Si}_{3} \mathrm{~N}_{4}$ gradient coating was applied at continuously varying temperatures. The major advantage of applying the $\mathrm{B}_{4} \mathrm{C}-\mathrm{Si}_{3} \mathrm{~N}_{4}$ gradient coating at continuously varying temperatures is that the materials at the bottom and top surfaces of this gradient coating can be applied at the same temperature as they would be applied as bulk materials, and that at the same time the far field stress does not increase. For the case of applying the entire $\mathrm{B}_{4} \mathrm{C}-\mathrm{Si}_{3} \mathrm{~N}_{4}$ gradient coating at $1000^{\circ} \mathrm{C}$, the stresses were of the same magnitude as for the application at continuously varying temperatures. However, the $\mathrm{Si}_{3} \mathrm{~N}_{4}$ in this coating was applied also at $1000^{\circ} \mathrm{C}$ and not at $1500^{\circ} \mathrm{C}$, at which the bulk $\mathrm{Si}_{3} \mathrm{~N}_{4}$ would be applied. This may result in an inferior quality of the coating, or may not be physically possible. The normal and shear stress in the far field were zero and are therefore not shown.

The through-the-thickness variation of the stresses at the beginning of the corner, location 2, are shown in Fig. 6.48. This is the location at which the largest tangential stresses in the $\mathrm{B}_{4} \mathrm{C}$ coating occurred for the discrete coating case (Fig. 6.43a). A comparison of the tangential stress in the far field (Fig. 6.47) and at the beginning of the corner (Fig. 6.48) shows that the largest tangential stress for the discrete coating case occurred in the $\mathrm{B}_{4} \mathrm{C}$ 
coating near the $\mathrm{B}_{4} \mathrm{C}$-substrate interface at the beginning of the corner, while the largest tangential stresses for the gradient coating cases occurred in the far field, at the interface between the two gradient coatings. As in the far field, at the beginning of the corner, application of the $\mathrm{B}_{4} \mathrm{C}-\mathrm{Si}_{3} \mathrm{~N}_{4}$ gradient coating at higher temperatures resulted in higher tangential stresses. In contrast to the far field, where the tangential stress in the $\mathrm{Si}_{3} \mathrm{~N}_{4}$ coating was tensile, the tangential stress in the $\mathrm{Si}_{3} \mathrm{~N}_{4}$ coating at the beginning of the corner was compressive at the free surface, and decreased in magnitude for locations further into the coating. The distribution of the axial stress $\bar{\sigma}_{z}$ at the beginning of the corner was very similar to the distribution of the axial stress in the far field. Recall that the axial stress only changes from one location to the next due to its Poisson coupling to the other stress components.

At the beginning of the corner, the distribution of the normal stress $\bar{\sigma}_{n}$ through the thickness of all coatings and the substrate was almost the same for all gradient coating cases, but it was different for the discrete coating case. The maximum normal stress for the gradient coating cases was only about $50 \%$ of the maximum normal stress for the discrete coating case. Thus, delamination of the coatings is less likely for gradient coatings.

The maximum shear stress at the beginning of the corner occurred near the interface between the two gradient coatings. As for the tangential stress, the maximum shear stress increased with increasing application temperatures of the $\mathrm{B}_{4} \mathrm{C}-\mathrm{Si}_{3} \mathrm{~N}_{4}$ gradient coating. For the continuously varying temperature application of the $\mathrm{B}_{4} \mathrm{C}-\mathrm{Si}_{3} \mathrm{~N}_{4}$ gradient coating, the maximum shear stress was lower than it was for the discrete coating case.

At the center of the corner, location 3, (Fig. 6.49), the overall distribution of the tangential stresses $\bar{\sigma}_{s}$ in the gradient coatings was similar to the distribution at the beginning of the corner and in the far field. However, the magnitude of the stress was smaller than at the other two locations. The same holds for the distribution of the tangential stress in the gradient coatings at the end of the corner and near the horizontal line of symmetry, shown in Figs. 6.50 and 6.51, respectively. However, the distribution of the tangential stress in the $\mathrm{Si}_{3} \mathrm{~N}_{4}$ coatings is quite different for the different locations. While this stress was tensile 
through the thickness of the coating in the far field, it was compressive throughout the coating at the center of the corner, and changed from a tensile stress at the free surface to a compressive stress at locations further into the coating at the end of the corner, and at the horizontal line of symmetry.

As at the beginning of the corner, at the center of the corner, and at the end of the corner, the normal stress $\bar{\sigma}_{\boldsymbol{n}}$ was very close for all gradient coating cases. Its maximum magnitude was lower for the gradient coating cases than it was for the discrete coating case. While the normal stress was tensile throughout the thickness of the coatings at the beginning of the corner, at the end of the corner it was compressive throughout the thickness of the coatings for the gradient coating cases.

The distribution of axial stress $\bar{\sigma}_{z}$ through the thickness of the coatings at all other locations was similar to the distribution of this stress in the far field.

At the center of the corner and at the end of the corner, the difference in the distribution of the shear stress $\bar{\tau}_{s n}$ among the various temperature scenarios for the gradient coating cases was very small. At both locations the maximum shear stress for the discrete coating case was larger than the maximum shear stress for any of the gradient coating cases. The magnitude of the maximum shear stress at the end of the corner was only about half the magnitude of the maximum shear stress at the center of the corner.

\subsection{Summary}

It was shown in this section that gradient coatings can be used to reduce stresses in a C/C substrate with multiple coatings. The stress concentration at the beginning of the corner can be significantly reduced if a gradient coating with a soft material is used near the $\mathrm{C} / \mathrm{C}$ substrate. Also, the normal and shear stress in the coatings were reduced for all gradient coating cases. The application temperature of the gradient coating had a significant influence on the maximum stresses in the coatings. For the particular coating cases discussed, application of the $\mathrm{B}_{4} \mathrm{C}-\mathrm{Si}_{3} \mathrm{~N}_{4}$ gradient coating at higher temperatures resulted in signifi- 


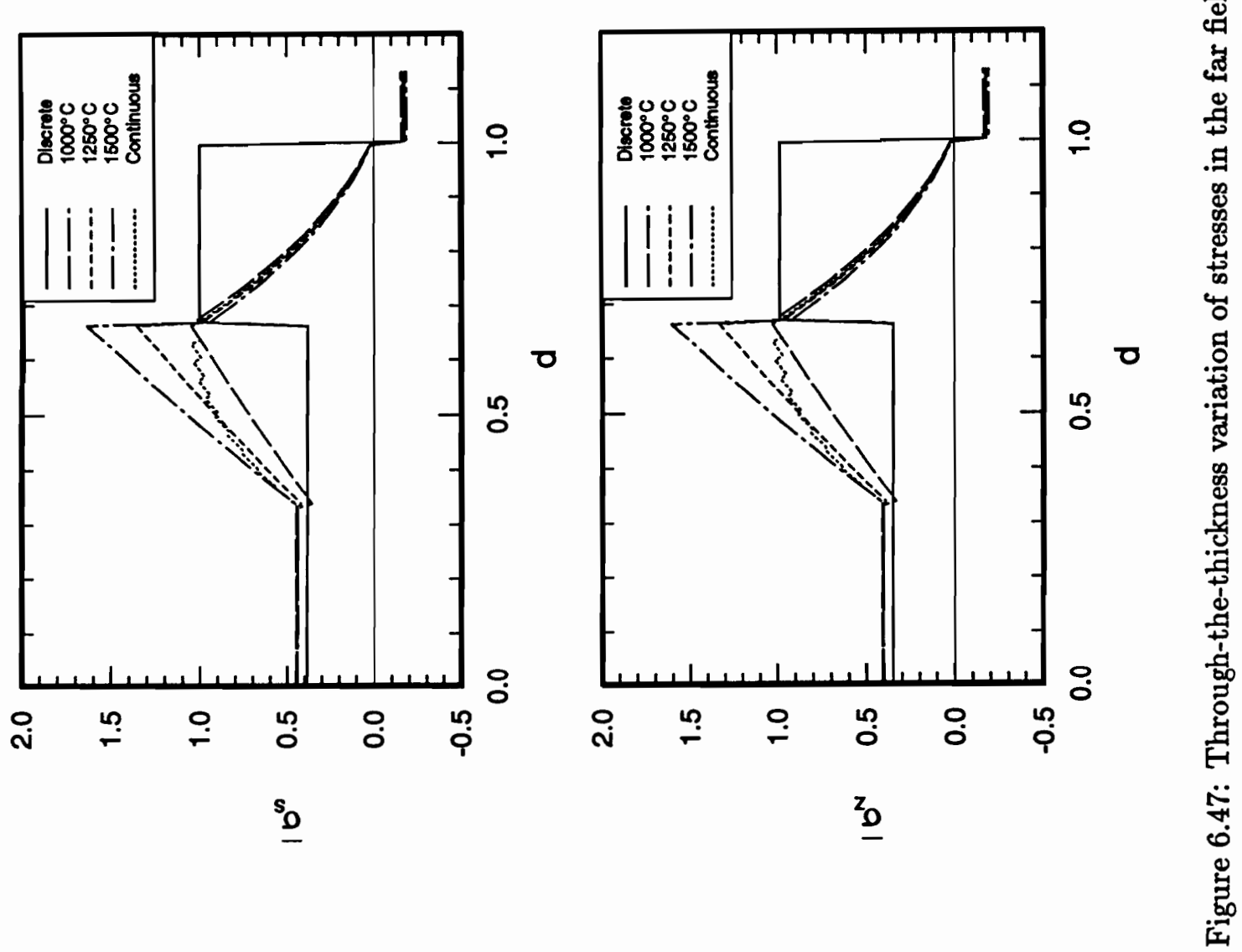




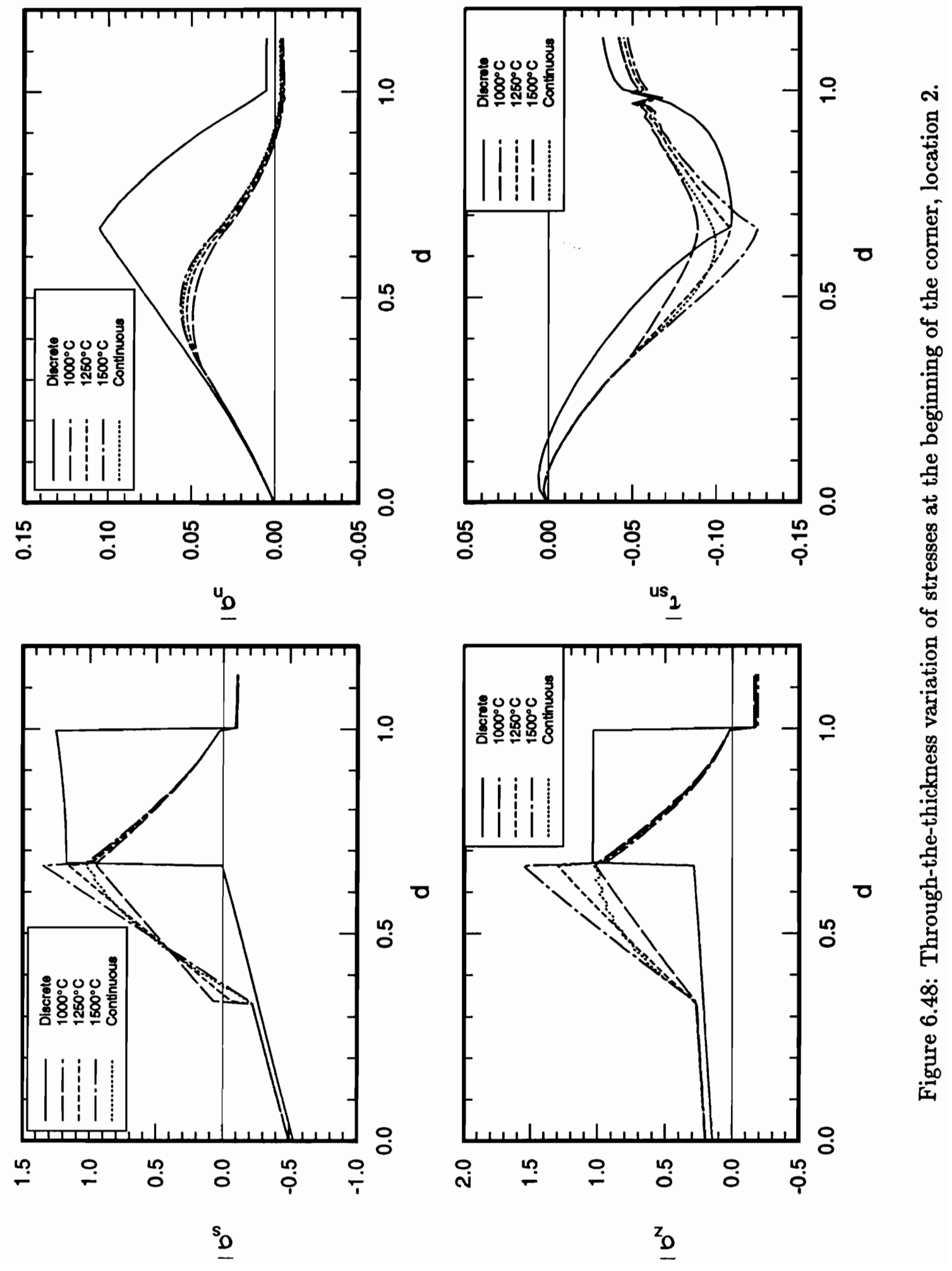




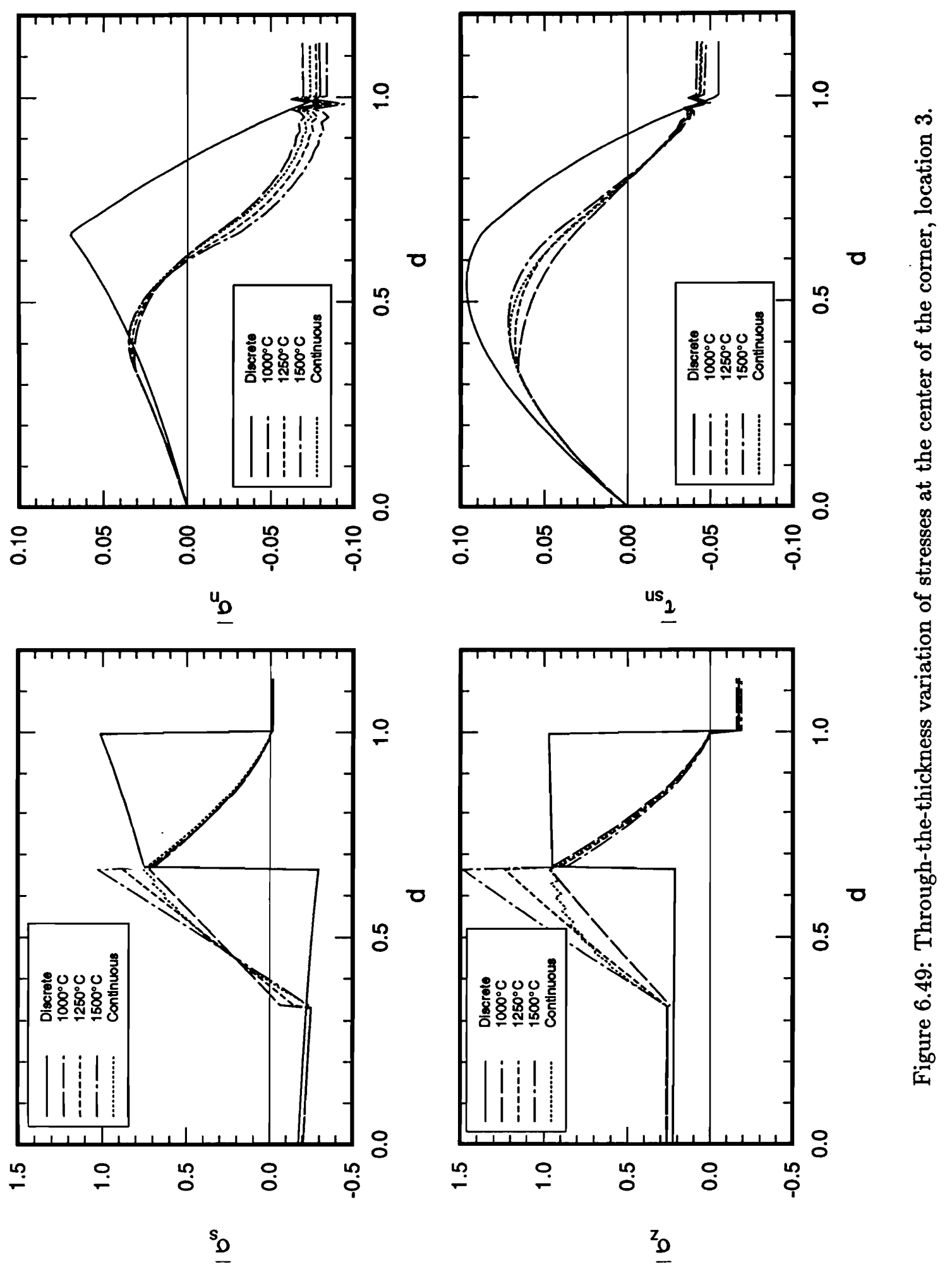




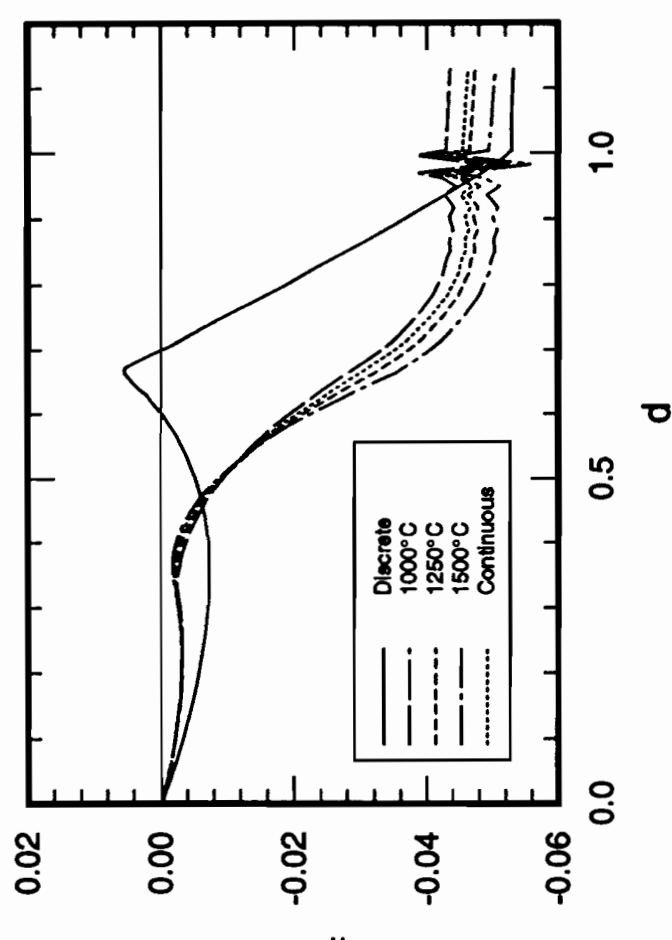

으

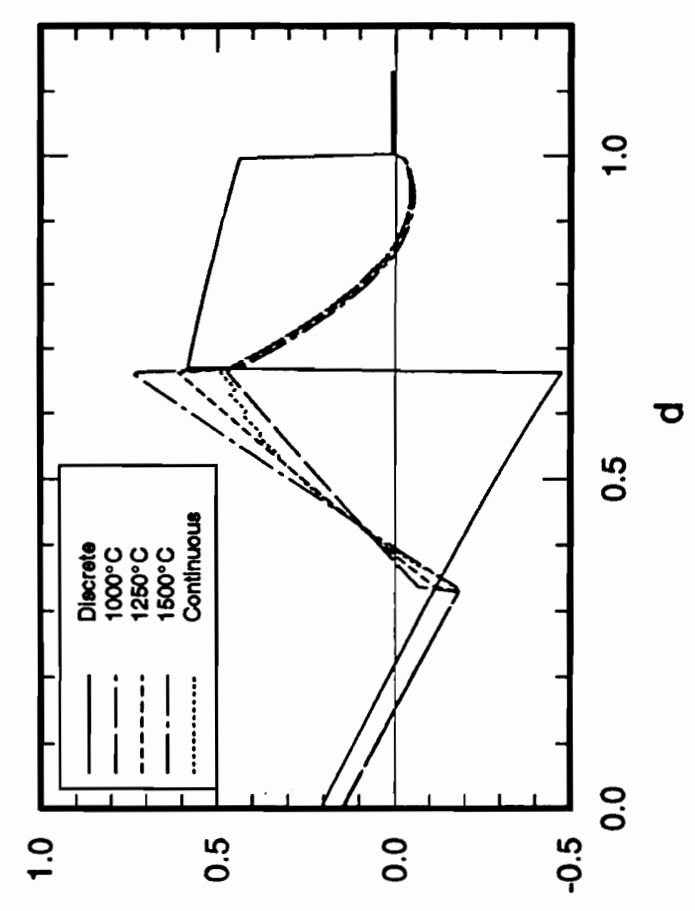

so
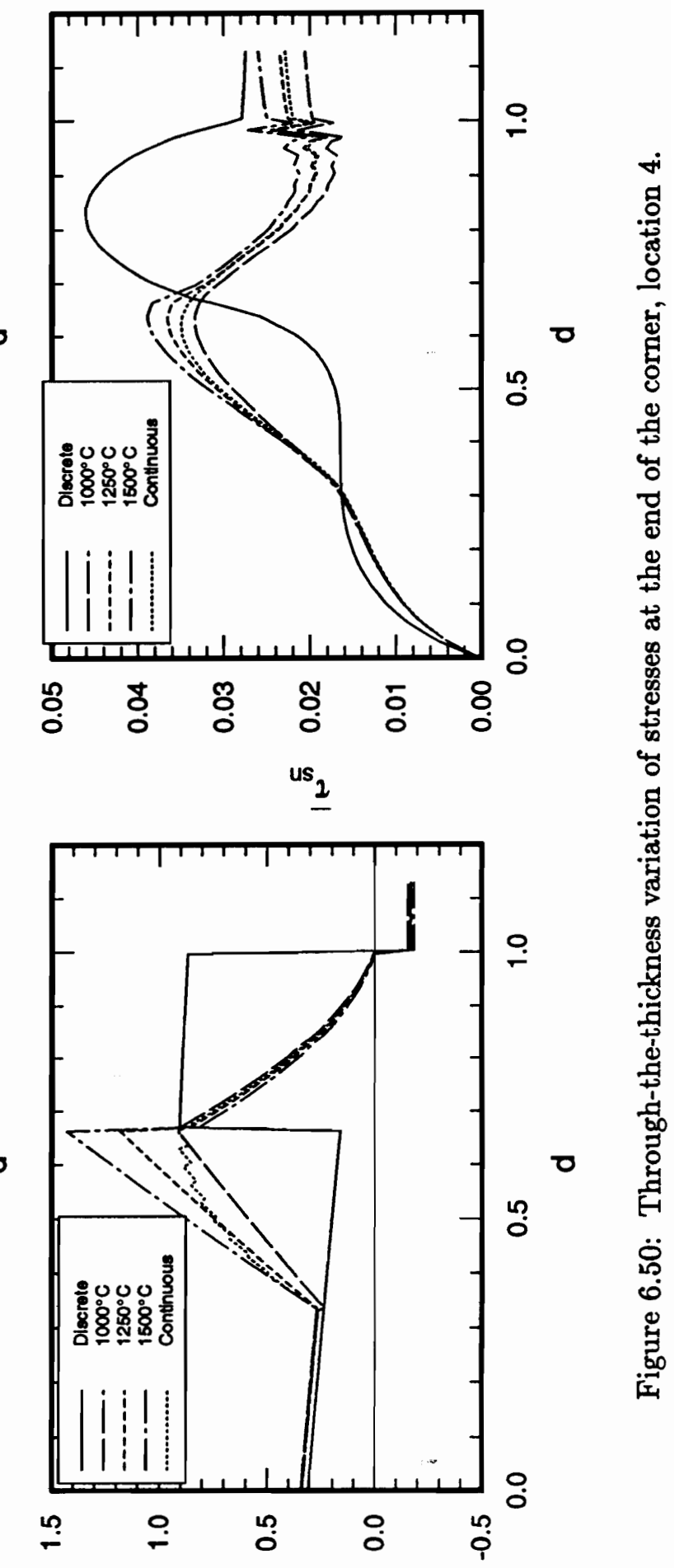

$\underline{2}$ 


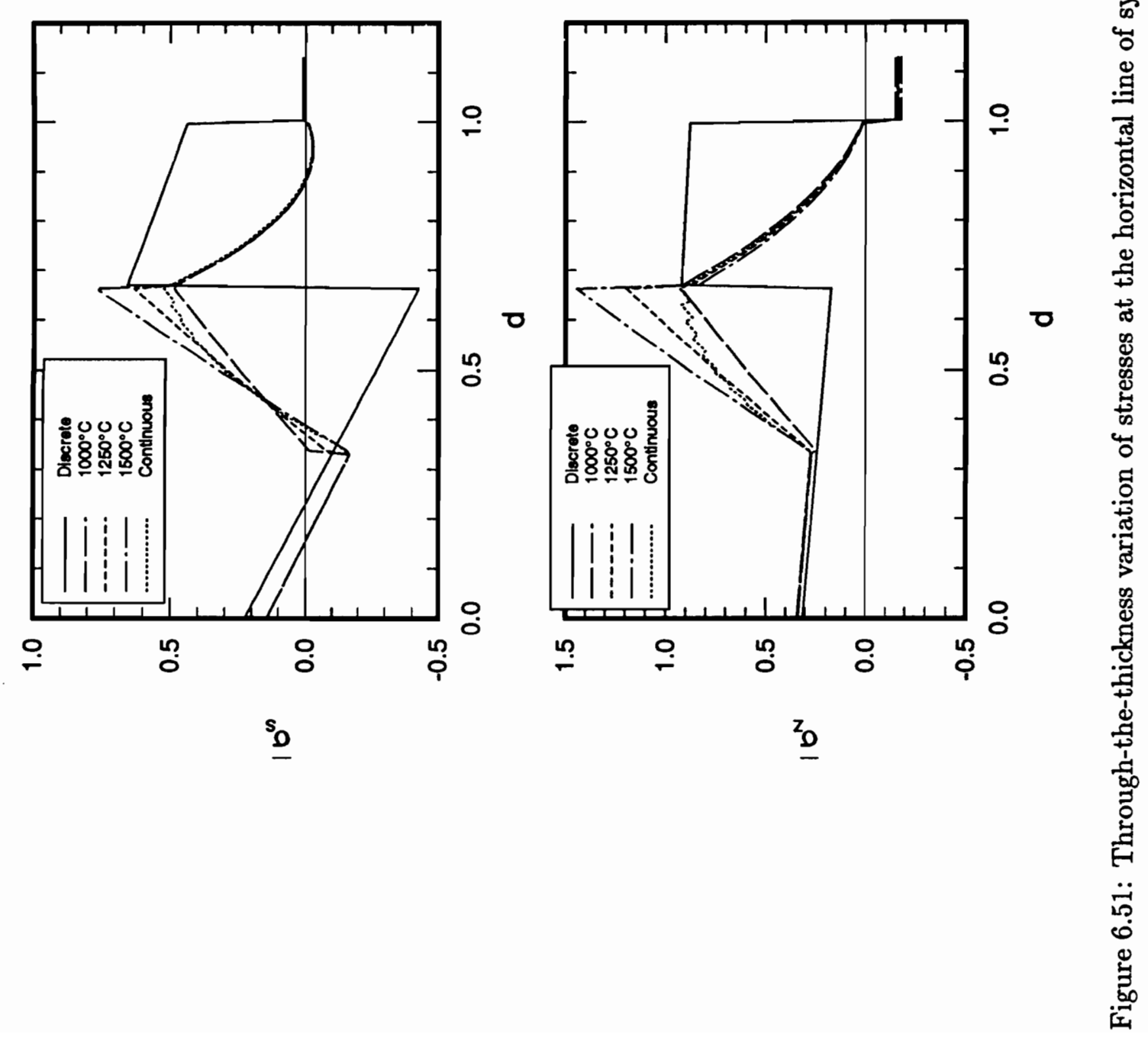


cantly increased far field stresses. It appeared to be best to apply the gradient coating by varying the temperature as the thickness and the composition of the coating change. In this case stresses in the coatings were reduced, and at the same time the coating material could be applied at a temperature at which the best coating quality could be obtained. 


\section{Chapter 7}

\section{Summary and Future Work}

\subsection{Summary}

The objective of this dissertation was to develop an analysis model to predict stresses in coatings on carbon-carbon composites and to study concepts for stress reduction in the coatings. The analysis tool of choice was an incremental generalized plane-strain finite element model that allowed for temperature-dependent material properties, application of coatings at different temperatures, and gradient coatings. The particular geometry of the $\mathrm{C} / \mathrm{C}$ substrate and the coatings considered in this work is shown in Fig. 1.3.

In addition to the finite element model, an incremental simplified plane-stress model was developed that could be used to predict stresses away from geometric discontinuities. Room temperature stresses in a $\mathrm{C} / \mathrm{C}$ substrate with a $\mathrm{SiC}$ coating and in a $\mathrm{C} / \mathrm{C}$ substrate with a $\mathrm{B}_{4} \mathrm{C}$ coating and $\mathrm{a} \mathrm{Si}_{3} \mathrm{~N}_{4}$ coating applied at different temperatures were analyzed in detail. Temperature-dependent material properties were shown to be important for accurate predictions of stresses (Figs. 6.4 - 6.5). For both coating cases large stress concentrations were present in the coatings in the corner region near the coating-substrate interface (Figs. 6.6 and 6.43). The magnitude of the stresses in those stress concentrations are larger than the magnitudes of stresses in the far-field, away from the corner. The normal stress in 
the coatings was confined to the corner region. It diminished rapidly away from the corner. Though the shear stress was also predominant in the corner region, it diminished much more slowly away from the corner.

The width of the substrate was shown to have a significant influence on the stress distribution for narrow substrates (Figs. 5.10 and 5.11). For wide substrates, the stress distribution was insensitive to changes in the width of the substrate. Far-field stresses for wide substrates were accurately predicted using an incremental simplified plane-stress analysis model (Table 5.2).

In the corner region, the stress distribution in the coatings was significantly influenced by the substrate orthotropy (Fig. 5.18). Most importantly, the region of influence of the corner region increased for orthotropic substrates compared to isotropic substrates. The stress concentrations in the corner region significantly increased with decreasing corner radius. Thus, as large as possible corner radii should be used.

The compensation/compliant layer concept was shown to be effective in reducing stress concentrations in the corner region. It was found that for constant total coating thickness, a thickness ratio of compliant layer thickness to oxidation protection coating thickness exists for which the largest tensile stresses in the coatings would reach a minimum value (Table 6.2). At this thickness the far-field tangential stress and the tangential stress in the stress concentration had the same value. In essence, the stress concentration had been eliminated. The effectiveness of the stress reduction of the compliant layer increased with increasing coefficient of thermal expansion of that layer (Table 6.3). In fact, an optimum combination of thickness and coefficient of thermal expansion of the compliant layer existed for which the largest tensile stresses in the oxidation protection coating would reach their minimum value (Fig. 6.14). The reduction in the largest stress was achieved without significantly increasing the other stress components.

An isotropic increase of the moduli of the compliant layer also resulted in reduced stresses in the oxidation protection coating; however, for larger coefficients of thermal expansion, stresses in the compliant layer reached magnitudes at which this layer would fail before 
the oxidation protection coating would fail (Figs. 6.20 and 6.21). Thus, for most effective stress reduction the compliant layer should have low moduli and high coefficients of thermal expansion. Large shearing of the compliant layer in the corner region, as shown in Fig. 6.12, appeared to be the cause for the stress reduction in the oxidation protection coating.

Orthotropic compliant coatings were less effective in reducing stresses than isotropic compliant coatings. In fact, for a quasi-isotropic compensating coating for which the modulus of elasticity was increased independently from the shear modulus, the stresses in the oxidation protection coating increased significantly compared to the case with no compensating coating (compare Fig. 6.28 to the first column in Table 6.2).

Similarly to inserting compensating/compliant layers, inserting gradient coatings in which the properties varied from those of a compliant layer at the gradient coating-substrate interface to those of the oxidation protection coating at the gradient coating-oxidation protection coating interface, resulted in reduced stresses in the oxidation protection coating. In fact, lower stresses would be achieved with a gradient coating than with a compensating/compliant coating (compare Table 6.4 and Table 6.2). For a given gradient coating to oxidation protection coating thickness ratio, a convex distribution of properties through the thickness of the gradient coating (eq. (6.2)) resulted in the lowest stresses in the coatings (Table 6.6).

For multiple coatings applied at different temperatures, the application temperature of the inner coating had a larger effect on the stresses in that coating. The higher the application temperature of that coating was, the higher the room temperature stresses were (Tables 6.10 and 6.11). Gradient coatings with property variations from those of a compliant layer to those of the oxidation protection layers were effective in reducing the stresses. If the "normal" application temperatures of the materials at the two surfaces of the gradient coatings were different, then it appeared best to vary the application temperature of the gradient coating with the composition of that coating. In that case application temperatures of the material in the coatings are close to the "normal" application temperatures of the material and at the same time stresses are significantly reduced in the coatings 
(Figs. 6.47 - 6.51).

In summary, the stress concentrations in the corner region in $\mathrm{C} / \mathrm{C}$ substrates with coatings would be significantly reduced by substituting a compensating/compliant layer or a gradient coating for part of the oxidation protection coating. Lowest stresses were achieved for a gradient coating with a convex distribution of material properties through the thickness. However, for constant total coating thickness neither gradient coatings nor compensating/compliant layers reduced stresses in the far-field away from the corner.

\subsection{Future Work}

Based on the observations made in the present research, below some suggestions for future work are outlined.

Due to the high stresses and high temperatures in the in the coatings, linear elastic material behavior may be an approximation, especially for compensating/compliant layers. Therefore it is suggested to extend the incremental constitutive law to account for material nonlinearities and plasticity. A constitutive law similar to the one described by Griffin [25] could be used.

The high shear and normal stresses at the coating interfaces may lead to delamination and/or sliding at those interfaces. This could be accounted for in a more sophisticated model. Also, cracking of the coatings and crack propagation of the coatings could be modeled. This would then show whether or not cracks that initiate within the coating would propagate to the surface. Because the $\mathrm{C} / \mathrm{C}$ composites might be exposed to high temperatures for large periods of time during service life and also may experience temperature gradients, modeling of creep and temperature gradients could be incorporated into the analysis. Ideally, the thermal stress analysis would be coupled with an analysis that predicts temperature distributions in the composite.

Finally, it would be interesting to obtain experimental results and compare those to results predicted by the finite element model. Eventually, this must be done. 


\section{Bibliography}

[1] J. Aboudi, S. M. Arnold, and M.-J. Pindera. Response of functionally graded composites to thermal gradients. Composites Engineering, 4(1):1-18, 1994.

[2] J. M. Allen. Effect of temperature dependent mechanical properties on thermal stress in cooled turbine blades. Journal of Engineering for Power, 104:349-353, 1982.

[3] anon. Engineering property data on selected ceramics. Technical Report MCIC HB-07, Metals and Ceramics Information Center, Batelle Columbus Division, 505 King Ave., Columbus, OH, 1976-1987.

[4] anon. ABAQUS, version 4.8. Technical report, copyright Hibbitt, Karlsson, and Sorensen, Inc., 1987.

[5] anon. Mathematica, version 2.2. Technical report, copyright Wolfram Research, Inc., 1993.

[6] F. J. Arendts. Aktuelle Entwicklungen in der Strukturtechnik. Zeitschrift für Flugwissenschaften und Weltraumforschung (in German), 16:231-246, 1992.

[7] S. M. Arnold, V. K. Arya, and M. E. Melis. Reduction of thermal residual stresses in advanced metallic composites based upon a compensating/compliant layer concept. Journal of Composite Materials, 26(9):1287-1309, 1993.

[8] W. B. Avery and C. T. Herakovich. A study of the mechanical behavior of a 2-D carboncarbon composite. Technical Report CCMS-87-13, Virginia Polytechnic Institute and State University, 1987.

[9] K. J. Bathe and E. L. Wilson. Numerical Methods in Finite Element Analysis. PrenticeHall, Inc., 1982.

[10] J. D. Buckley and D. D. Edie, editors. Carbon-Carbon Materials and Composites, NASA Reference Publication 1254, February 1992.

[11] J. K. Chen, C. T. Sun, and C. I. Chang. Failure analysis of graphite/epoxy laminate subjected to combined thermal and mechanical load. Journal of Composite Materials, 19(5):408-423, 1985. 
[12] L.-W. Chen and L.-Y. Chen. Thermal buckling behavior of laminated composite plates with temperature-dependent properties. Composite Structures, 13:275-287, 1989.

[13] L.-W. Chen and L.-Y. Chen. Thermal postbuckling behaviors of laminated composite plates with temperature dependent properties. Composite Structures, 19:267-283, 1991.

[14] N. Cherradi, A. Kawasaki, and M. Gasik. Worldwide trends in functional graded materials research and development. Composites Engineering, 4(8):883-894, 1994.

[15] P. D. Copp, J. C. Dendis, and S. Mall. Failure analysis and damage initiation in carbon-carbon composite materials under three-point bending. Journal of Composite Materials, 25:593-608, May 1991.

[16] H. L. Crane Jr., N. E. Gibbs, W. G. Poole Jr., and P. K. Stockmeyer. Matrix bandwidth and profile reduction. ACM Transactions on Mathematical Software, 2(4):375-377, December 1976.

[17] D. C. Cranmer, editor. Fundamentals of Carbon/Carbon Workshop, National Institute of Standards and Technology, Gaithersburg, Maryland, December 6-7 1990.

[18] J. T. Drake, R. L. Williamson, and B. H. Rabin. Finite element analysis of thermal residual stresses at graded ceramic-metal interfaces. Part 2. Interface optimization for residual stress reduction. Journal of Applied Physics, 74(2):1321-1326, July 1993.

[19] J. M. Duva, B. R. Olson, and H. N. G. Wadley. Functionally graded materials for yield suppression at stress concentrations. Composites Engineering, 4(1):107-114, 1994.

[20] E. Fitzer, editor. Carbon Fibers and Their Composites, chapter 1, pages 3-45. Springer Verlag, 1985.

[21] T. Fuchiyama, N. Noda, T. Tsuji, and Y. Obata. Analysis of thermal stress and stress intensity factor of functionally gradient materials. In Holt et al. [29], pages 425-432.

[22] S. M. Gee and J. A. Little. Oxidation behaviour and protection of carbon/carbon composites. Journal of Materials Science, 26:1093-1100, 1991.

[23] C. G. Goetzel. High-temperature properties of some carbon-carbon composites. High Temperature - High Pressure, 12:11-22, 1980.

[24] O. H. Griffin Jr. Three-dimensional curing stresses in symmetric cross-ply laminates with temperature-dependent properties. Journal of Composite Materials, 17:449-463, September 1983.

[25] O. H. Griffin Jr., M. P. Kamat, and C. T. Herakovich. Three-dimensional inelastic finite element analysis of laminated composites. Technical Report VPI-E-80-28, Virginia Polytechnic Institute and State University, 1980. 
[26] H. T. Hahn and N. J. Pagano. Curing stresses in composite laminates. Journal of Composite Materials, 9:91-106, January 1975.

[27] W. Hallmann and W. Ley, editors. Handbuch der Raumfahrttechnik, chapter 6, pages 167-170. Springer Verlag, 1985.

[28] G. K. Haritos and O. O. Ochoa, editors. Damage and Oxidation Protection in High Temperature Composites, volume 1, 1991.

[29] J. B. Holt, M. Koizumi, T. Hirai, and Z. A. Munir, editors. Functionally Gradient Materials, volume 34 of Ceramic Transactions, 1993.

[30] T. R. Hsu. The Finite Element Method in Thermomechanics. Allen \&. Unwin, Inc., Boston, 1986.

[31] W. Huettner, R. Weiss, G. Dietrich, and R. Meistring. Oxidation inhibited carbon/carbon - A candidate material for hot structures. In ESA Symposium: Space Applications of Advanced Materials, ESA SP-303, pages 91-95, Noordwijk (NL), 21-23 March 1990 June 1990.

[32] K. Jayaraman and K. L. Reifsnider. The interface in unidirectional fiber-reinforced epoxies: Effect on residual thermal stresses. Composites Science and Technology, 47(2):119-129, 1993.

[33] R. M. Jones. Mechanics of Composite Materials. Hemisphere Publishing Corporation, New York, 1975.

[34] M. A. Kalam. Modified Rayleigh-Ritz method in nonaxisymmetric thermoelastic orthotropic hollow cylinder with temperature dependent mechanical proporties. Journal of Thermal Stresses, 4(1):31-38, 1981.

[35] N. Kamiya and A. Fukui. Finite deflection and postbuckling behavior of heated rectangular plates with temperature dependent properties. Nuclear Engineering and Design, 72:415-420, 1982.

[36] T. Kinjo, S. Maekawa, and T. Kobayashi. Development of carbon-carbon composites for primary structure and thermal protection system of space vehicle. In Proceedings of the Sixth Japan-U.S. Conference on Composite Materials, pages 279-286, June 22-24 1992.

[37] M. Koizumi. The concept of FGM. In Holt et al. [29], pages 3-10.

[38] W. Kowbel. The mechanism of oxidation protection of $\mathrm{C} / \mathrm{C}$ composites coated with graded-codeposited carbides and nitrides. In Holt et al. [29], pages 232-244.

[39] W. Kowbel, M. Muszynski, and H. T. Tsou. Oxidation protection of C/C composites by graded-codeposited CVD coatings. In Haritos and Ochoa [28], pages 65-76. 
[40] W. Kowbel, M. Muszynski, and H. T. Tsou. Strengthening and toughening mechanisms in SiC/C CVD composites. In Haritos and Ochoa [28], pages 93-101.

[41] J. J. Lannutti. Functionally graded materials: Properties, potential and design guidelines. Composites Engineering, 4(1):81-94, 1994.

[42] S. G. Lekhnitskii. Theory of Elasticity of an Anisotropic Body. Mir Publishers, Moscow, 1981.

[43] S. Y. Limaye. Low thermal expansion (NZP) ceramic materials for oxidation protection coatings. In Cranmer [17], pages 494-519.

[44] R. A. Lucheta. Thermally induced interfacial stresses in a thin film on an infinite substrate. Applied Optics, 30(16):2252-2256, June 1991.

[45] H. Mahfuz, , D. Xue, S. Jeelani, D. Baker, and S. Johnson. Nonlinear response of 2-D carbon-carbon composites by layered shell elements. In Developments in Theoretical and Applied Mechanics, volume XVI:III.I, pages 62-72, December 6-7 1992.

[46] H. Mahfuz, P. S. Das, D. Xue, J. Krishnagopalan, and S. Jeelani. A combined experimental and finite element study to predict the failure mechanisms in $\mathrm{SiC}$ coated carbon-carbon composites at room and elevated temperatures under flexural loading. Journal of Reinforced Plastics and Composites, 12:825-842, July 1993.

[47] H. L. N. McManus and G. S. Springer. High temperature thermomechanical behavior of carbon-phenolic and carbon-carbon composites, I. Analysis. Journal of Composite Materials, 26(2):206-229, 1993.

[48] H. L. N. McManus and G. S. Springer. High temperature thermomechanical behavior of carbon-phenolic and carbon-carbon composites, II. Results. Journal of Composite Materials, 26(2):230-255, 1993.

[49] G. W. Meetham. High-temperature materials - a general review. Journal of Materials Science, 26:853-860, 1991.

[50] M. Mendelson and T. McKechnie. Functionally gradient thermal barrier coatings: Design. In Holt et al. [29], pages 417-424.

[51] Y. Mikata and M. Taya. Stress field in a coated continuous fiber composite subjected to thermo-mechanical loadings. Journal of Composite Materials, 19:554-578, 1985.

[52] T. Ngai. Carbon-carbon composites. In S. M. Lee, editor, International Encyclopedia of Composites, pages 158-187. VCH New York, 1990.

[53] N. Noda. Thermal stresses in materials with temperature- dependent properties. Applied Mechanics Reviews, 44(9):383-397, September 1991. 
[54] A. K. Noor and W. S. Burton. Computational models for high-temperature multilayered composite plates and shells. Applied Mechanics Reviews, 45(10):419-446, October 1992.

[55] Z. S. Olesiak. Influence of surface heating on coated elastic solids. Journal of Thermal Stresses, 12:293-303, 1989.

[56] J. Padovan, D. Dougherty, and R. Hendricks. Inelastic high-temperature thermomechanical response of ceramic coated gas turbine seals. Journal of Thermal Stresses, 9(1):31-43, 1986.

[57] J. Padovan, D. Dougherty, R. Hendricks, M. J. Braun, and B. T. F. Chung. High temperature thermomechanical analysis of ceramic coatings. Journal of Thermal Stresses, $7(1): 51-74,1984$.

[58] J. Padovan and Y. Xiam. Analysis of thermomechanical oxidation fields in thermal barrier coatings. Journal of Thermal Stresses, 9:251-277, 1986.

[59] D. C. Pardo and O. O. Ochoa. Thermomechanical response of inhibited carbon-carbon composites. In Proceedings of the American Society for Composites. Ninth Technical Conference, pages 47-54. Technomic Inc., Lancaster, September 20-22 1994.

[60] M.-J. Pindera, T. O. Williams, and S. M. Arnold. Thermoplastic response of metalmatrix composites with homogenized and functionally graded interfaces. Composites Engineering, 4(1):129-145, 1994.

[61] J. N. Reddy. An Introduction to the Finite Element Method. McGraw-Hill Book Company, 1984.

[62] L. Rubin. Applications of Carbon-Carbon. In Buckley and Edie [10], pages 267-281.

[63] A. Sakamoto. Status of R\&D project on high-performance materials for severe environments. In Proceedings of the Sixth Japan-U.S. Conference on Composite Materials, pages 271-278, Jüie 22-24 1992.

[64] G. Savage. Carbon-Carbon Composites. Chapman \& Hall, 1993.

[65] J. E. Sheehan. Coatings for carbon-carbon oxidation protection. In Cranmer [17], pages 250-265.

[66] J. E. Sheehan. High-Temperature Coatings on Carbon Fibers and Carbon-carbon Composites. In Buckley and Edie [10], pages 223-266.

[67] I. Shiota and R. Watanabe. Fabrication and properties of FGM. In Advanced Composite Materials II, pages 96-104, 1991. 
[68] Y. Soda, Y. Kuda, S. Uemura, T. Saitoh, Y. Wakamatsu, and M. Niino. Carbon/Carbon composites coated with $\mathrm{SiC} / \mathrm{C}$ functionally gradient materials. In Holt et al. [29], pages 125-132.

[69] J. R. Strife. Fundamentals of protective coating strategies for carbon-carbon composites. In Haritos and Ochoa [28], pages 121-130.

[70] T. Suemitsu, Y. Matsuzaki, J. Fujioka, M. Uchida, Y. Sohda, Y. Kude, S. Uemura, Y. Kuroda, S.Ueda, and A. Moro. Carbon/Carbon composites for combustors of space planes. In Holt et al. [29], pages 315-322.

[71] C. M. Taylor and R. C. Bill. Thermal stresses in plasma-sprayed ceramic gas path seal. Journal of Aircraft, 16(4):239-246, 1979.

[72] L. S. Theibert. The Air Force perspective on the needs for oxidation resistant carboncarbon composites. In Cranmer [17], pages 1-23.

[73] A. Touati, J. M. Roelandt, F. Armanet, and G. Béranger. Simulation of the behaviour of high temperature oxidized structures (application to coatings). Composite Materials, pages 233-238, 1992.

[74] Y. Ueda and T. Yamakawa. Thermal nonlinear behavior of structures. In Advances in Computational Methods in Structural Mechanics and Design. The University of Alabama, Huntsville, Alabama, 1972.

[75] K. Washizu. Variational Methods in Elasticity $\mathcal{E}$ Plasticity. Pergamon Press, 1982.

[76] R. L. Williamson and B. H. Rabin. Numerical modeling of residual stress in $\mathrm{Ni}^{-} \mathrm{Al}_{2} \mathrm{O}_{3}$ gradient materials. In Holt et al. [29], pages 55-65.

[77] R. L. Williamson, B. H. Rabin, and J. T. Drake. Finite element analysis of thermal residual stresses at graded ceramic-metal interfaces. Part 1. Model description and geometrical effects. Journal of Applied Physics, 74(2):1310-1320, July 1993.

[78] T. Yamada, Y. Kojima, N. Ohnaka, and M. Chigasaki. Finite element analysis of residual thermal stress in $\mathrm{SiC} /(\mathrm{C} / \mathrm{C})$ laminates with controlled compositional gradients. Japanese Society of Materials Science, Journal (in Japanese), 40:1029-1034, 1991. 


\section{Appendix A}

\section{Temperature-Dependent Material}

\section{Properties}

In this appendix the temperature-dependent material properties are given. The required properties for the finite element model are the moduli of elasticity, $E_{1}, E_{2}$, and $E_{3}$, the Poisson ratios, $\nu_{23}, \nu_{13}$, and $\nu_{12}$, the shear modules $G_{12}$, and the coefficients of thermal expansion, $\alpha_{1}, \alpha_{2}$, and $\alpha_{3}$. Here conventional subscript notation is used with respect to a principal material 1-2-3 coordinate system. The application temperatures for the coatings are given in Table A.1.

The temperature-dependent material data for the orthotropic carbon-carbon substrate was estimated from figures given by Huettner [31] and Goetzel [23]. The moduli of elasticity $E_{1}, E_{2}$, and $E_{3}$, and the Poisson ratios $\nu_{23}, \nu_{13}$, and $\nu_{12}$ for the $\mathrm{C} / \mathrm{C}$ substrate are given as functions of temperature in Table A.2. The coefficients of thermal expansion $\alpha_{1}, \alpha_{2}$, and $\alpha_{3}$ are given in Table A.3. The shear modulus $G_{12}$ is constant for all temperatures at $G_{12}=17.9 \mathrm{GPa}$. The temperature-interpolated properties for the moduli of elasticity, the temperature-interpolated coefficients of thermal expansion, and the thermal strains obtained by integrating the coefficients of thermal expansion for a reference temperature of $1000^{\circ} \mathrm{C}$ for the substrate are shown in Fig. A.1. It should be noted here that in the 
Table A.1: Application temperatures for coatings.

\begin{tabular}{|c|c|c|c|c|}
\hline Material & Carbon & $\mathrm{B}_{4} \mathrm{C}$ & $\mathrm{SiC}$ & $\mathrm{Si}_{3} \mathrm{~N}_{4}$ \\
\hline $\mathrm{T}\left[{ }^{\circ} \mathrm{C}\right]$ & 1000 & 1000 & 1200 & 1500 \\
\hline
\end{tabular}

Table A.2: Temperature-dependent moduli of elasticity and Poisson ratios for C/C substrate.

\begin{tabular}{|r|c|c|c|c|c|c|}
\hline $\mathrm{T}\left[{ }^{\circ} \mathrm{C}\right]$ & $E_{1}[\mathrm{GPa}]$ & $E_{2}[\mathrm{GPa}]$ & $E_{3}[\mathrm{GPa}]$ & $\nu_{23}$ & $\nu_{13}$ & $\nu_{12}$ \\
\hline 0. & $100.0^{*}$ & $5.0^{*}$ & $100.0^{*}$ & $0.01^{*}$ & $0.07^{*}$ & $0.2^{*}$ \\
200. & 100.0 & 4.7 & 100.0 & 0.094 & 0.07 & 0.2 \\
400. & 101.0 & 4.4 & 101.0 & 0.008713 & 0.07 & 0.2 \\
500. & 103.0 & 4.2 & 103.0 & 0.008350 & 0.07 & 0.2 \\
600. & 105.0 & 4.0 & 105.0 & 0.007619 & 0.07 & 0.2 \\
700. & 107.0 & 3.8 & 107.0 & 0.007103 & 0.07 & 0.2 \\
800. & 108.0 & 3.6 & 108.0 & 0.006667 & 0.07 & 0.2 \\
900. & 109.0 & 3.4 & 109.0 & 0.006239 & 0.07 & 0.2 \\
1000. & 109.5 & 3.2 & 109.5 & 0.005845 & 0.07 & 0.2 \\
1200. & 110.0 & 3.0 & 110.0 & 0.005455 & 0.07 & 0.2 \\
\hline
\end{tabular}

present study the principal material direction coordinate system (1-2-3 coordinate system) for the substrate is aligned with the global $x-y-z$ coordinate system indicated in Fig. 5.1. The fibers in the substrate are parallel to the $x$ - and $z$-axes.

The properties for the isotropic $\mathrm{SiC}, \mathrm{B}_{4} \mathrm{C}, \mathrm{Si}_{3} \mathrm{~N}_{4}$, and pyrolytic carbon coatings are given in tabular form in Tables A.4 - A.6. These properties were taken from reference [3]. The material properties for the isotropic pyrolytic carbon coating were temperature-independent. These properties are given in Table A.7.

The room temperature material properties used in Chapter 5 and for the studies comparing temperature-dependent and temperature-independent analyses in Chapter 6 are indicated by $a *$ in the tables. 
Table A.3: Temperature-dependent coefficient of thermal expansion for $\mathrm{C} / \mathrm{C}$ substrate.

\begin{tabular}{|r|c|c|c|}
\hline $\mathrm{T}\left[{ }^{\circ} \mathrm{C}\right]$ & $\alpha_{1}\left[10^{6} /{ }^{\circ} \mathrm{C}\right]$ & $\alpha_{2}\left[10^{-6} /{ }^{\circ} \mathrm{C}\right]$ & $\alpha_{3}\left[10^{6} /{ }^{\circ} \mathrm{C}\right]$ \\
\hline 20. & $0.1^{*}$ & $4.8^{*}$ & $0.1^{*}$ \\
250. & -0.2 & 5.7 & -0.2 \\
500. & -0.5 & 6.7 & -0.5 \\
800. & 0.0 & 7.5 & 0.0 \\
1000. & 1.0 & 7.7 & 1.0 \\
1250. & 1.7 & 8.2 & 1.7 \\
1500. & 2.0 & 8.3 & 2.0 \\
2000. & 2.2 & 8.4 & 2.2 \\
\hline
\end{tabular}

Table A.4: Temperature-dependent properties for SiC.

\begin{tabular}{|r|c|c|c||r|c|}
\hline $\mathrm{T}\left[{ }^{\circ} \mathrm{C}\right]$ & $E[\mathrm{GPa}]$ & $\nu$ & $G[\mathrm{GPa}]$ & $\mathrm{T}\left[{ }^{\circ} \mathrm{C}\right]$ & $\alpha\left[10^{-6} /{ }^{\circ} \mathrm{C}\right]$ \\
\hline 0. & $480 .^{*}$ & $0.18^{*}$ & $203.39^{*}$ & 200. & $5.4^{*}$ \\
937. & 270. & 0.18 & 114.41 & 1000. & 6.1 \\
1400. & 370. & 0.18 & 156.78 & & \\
\hline
\end{tabular}

Table A.5: Temperature-dependent properties for $\mathrm{B}_{4} \mathrm{C}$.

\begin{tabular}{|r|c|c|c||r|c|}
\hline $\mathrm{T}\left[{ }^{\circ} C\right]$ & $E[\mathrm{GPa}]$ & $\nu$ & $G[\mathrm{GPa}]$ & $\mathrm{T}\left[{ }^{\circ} C\right]$ & $\alpha\left[10^{-6} /{ }^{\circ} \mathrm{C}\right]$ \\
\hline 20. & $289 . .^{*}$ & $0.19^{*}$ & $121.43^{*}$ & 600. & $4.3^{*}$ \\
1200. & 277. & 0.19 & 121.43 & 1000. & 6.1 \\
\hline
\end{tabular}

Table A.6: Temperature-dependent properties for $\mathrm{Si}_{3} \mathrm{~N}_{4}$.

\begin{tabular}{|r|c|c|c||r|c|}
\hline $\mathrm{T}\left[{ }^{\circ} C\right]$ & $E[\mathrm{GPa}]$ & $\nu$ & $G[\mathrm{GPa}]$ & $\mathrm{T}\left[{ }^{\circ} \mathrm{C}\right]$ & $\alpha\left[10^{-6} /{ }^{\circ} \mathrm{C}\right]$ \\
\hline 20. & $300 *$ & $0.22^{*}$ & $122.95^{*}$ & $\begin{array}{r}100 . \\
1000 .\end{array}$ & $\begin{array}{c}1.9^{*} \\
3.1\end{array}$ \\
\hline
\end{tabular}

Table A.7: Properties for pyrolytic carbon.

\begin{tabular}{|c|c|c|c|}
\hline $\mathrm{E}[\mathrm{GPa}]$ & $\nu$ & $\mathrm{G}[\mathrm{GPa}]$ & $\alpha\left[10^{-6} /{ }^{\circ} \mathrm{C}\right]$ \\
\hline 10.0 & 0.18 & 4.237 & 3.0 \\
\hline
\end{tabular}




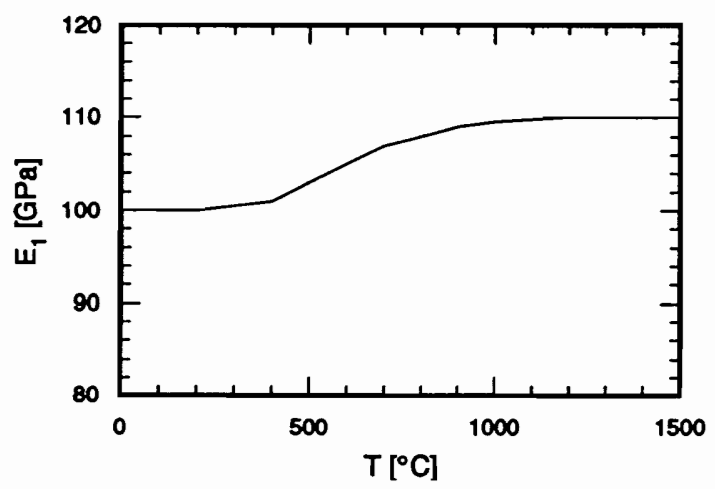

(a)

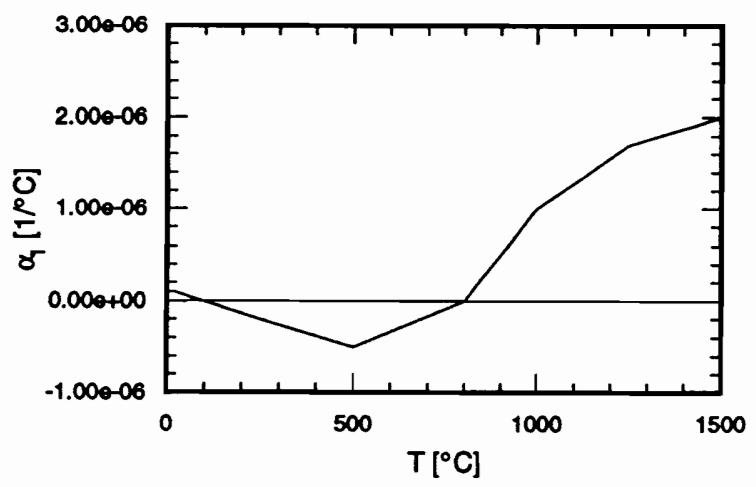

(c)

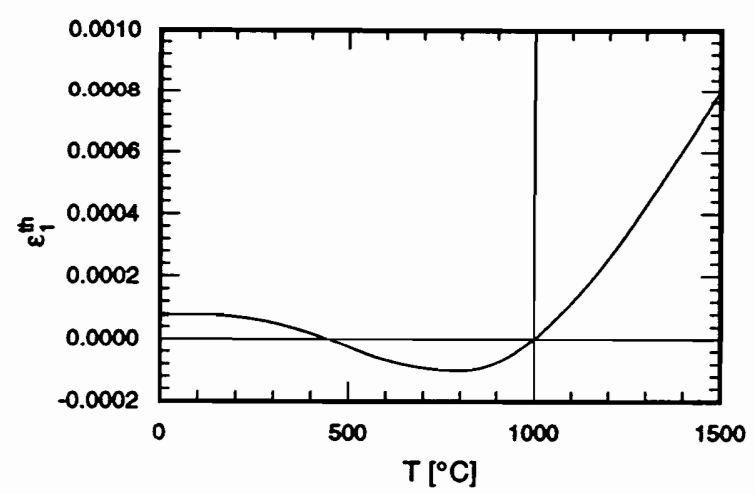

(e)

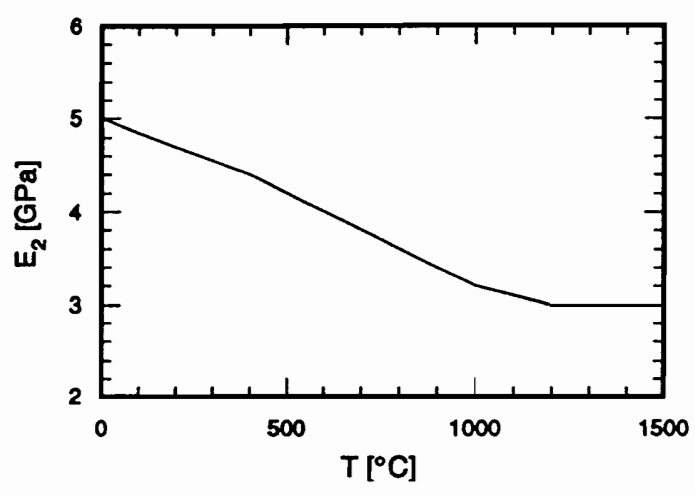

(b)

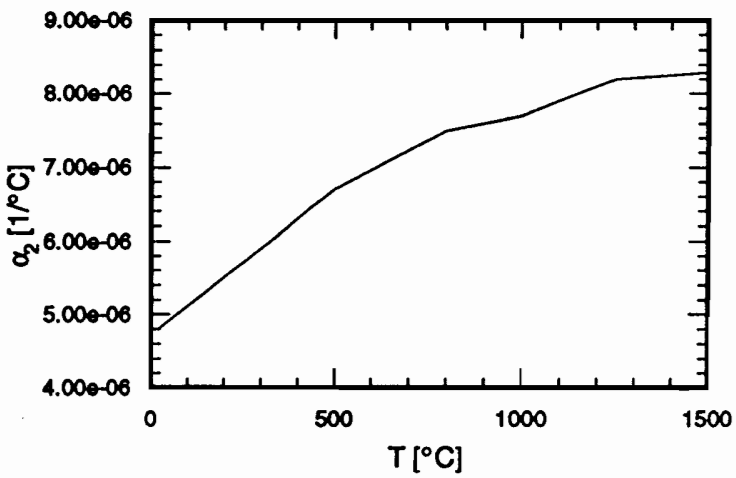

(d)

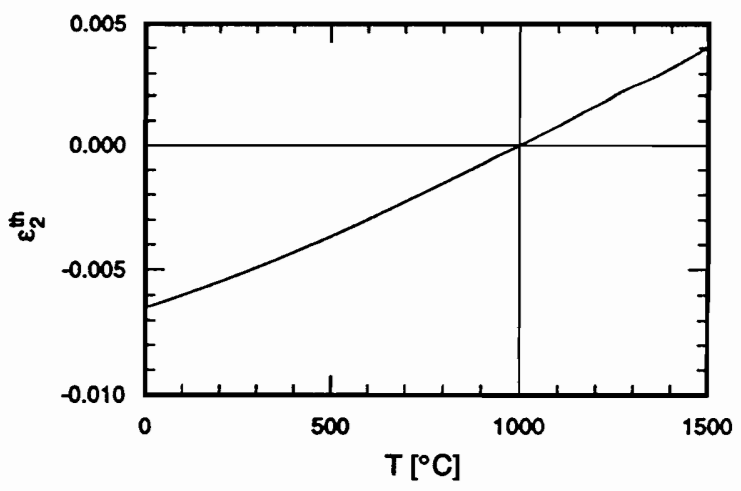

(f)

Figure A.1: Temperature-dependent properties for a $\mathrm{C} / \mathrm{C}$ substrate. 


\section{Appendix B}

\section{Comparison of Incremental}

\section{Constitutive Equations}

In this appendix a simple problem is analyzed to demonstrate the difference between the incremental constitutive equations derived in Section 2.1.1 and those derived in Section 2.1.2, and to demonstrate the importance of the coupling term in these incremental constitutive equations.

The problem considered is a clamped-clamped beam (Fig. B.1) with temperature-dependent material properties, subjected to a uniform thermal load. The beam is assumed stress and strain free at $0^{\circ} \mathrm{C}$, and stresses are to be calculated between $0^{\circ} \mathrm{C}$ and $1000^{\circ} \mathrm{C}$, every $100^{\circ} \mathrm{C}$.

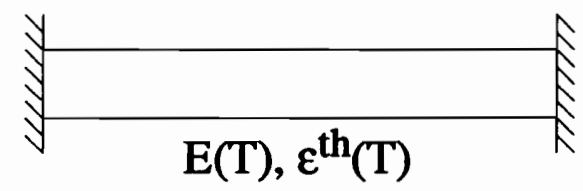

Figure B.1: Clamped-clamped beam. 
The linear thermoelastic constitutive equation for this problem is

$$
\sigma(T)=E(T)\left[\epsilon(T)-\epsilon^{t h}(T)\right]
$$

The incremental constitutive equation derived from the difference in stress states at two different temperatures (eq. (2.17)) reduces to

$$
\Delta \sigma=E\left(T_{n}\right)\left[\Delta \epsilon-\Delta \epsilon^{t h}-\Delta S \sigma\left(T_{n-1}\right)\right]
$$

and the incremental constitutive equations based on an assumed functional form (eq. (2.6)) reduces to

$$
\Delta \sigma=E\left(T_{n}\right)\left[\Delta \epsilon-\alpha \Delta T-\frac{\partial S}{\partial T} \sigma\left(T_{n-1}\right) \Delta T\right]
$$

With the clamped-clamped boundary conditions the exact solution to the problem can be obtained by setting $\epsilon=0$ in eq. (B.1) as

$$
\sigma(T)=-E(T) \epsilon^{t h}(T)
$$

The solution using either incremental constitutive equation can be obtained by setting $\Delta \epsilon=0$ and solving for $\Delta \sigma$. For each temperature increment the total stress in the beam can be obtained by updating the existing total stress as

$$
\sigma\left(T_{n}\right)=\sigma\left(T_{n-1}\right)+\Delta \sigma
$$

For the first temperature increment, $\sigma\left(T_{0}=0\right)=0$.

For illustrative purposes the following fictitious material properties were used:

$$
\begin{gathered}
E(T)=1000\left(T e^{-\frac{T}{100}}+250\right) \\
\epsilon^{t h}(T)=10^{-4}(\sqrt{T+20}-\sqrt{20}) .
\end{gathered}
$$

In Table B.1 the stresses calculated using the exact solution and the incremental constitutive equation eq. (B.2) are compared. To calculate results using the incremental constitutive equations $100^{\circ} \mathrm{C}$ temperature increments were used. As can be seen in Table B.1, the incremental constitutive equation eq. (B.2) yields exact results. 
Table B.1: Comparison of the stresses calculated using the exact solution and the incremental constitutive equations based on the difference in stress states at two different temperatures.

\begin{tabular}{|c|c|c|}
\hline \multirow{2}{*}{$\mathrm{T}\left[{ }^{\circ} \mathrm{C}\right]$} & \multicolumn{2}{|c|}{$\sigma[\mathrm{MPa}]$} \\
\cline { 2 - 3 } & Exact & eq. (B.2) \\
\hline 0 & 0 & 0 \\
100 & -185.905 & -185.905 \\
200 & -287.049 & -287.049 \\
300 & -355.449 & -355.449 \\
400 & -412.282 & -412.282 \\
500 & -464.46 & -464.46 \\
600 & -513.73 & -513.73 \\
700 & -560.444 & -560.444 \\
800 & -604.736 & -604.736 \\
900 & -646.771 & -646.771 \\
1000 & -686.757 & -686.757 \\
\hline
\end{tabular}

As was mentioned in the discussion in Section 2.1.1, when incremental constitutive equations are derived from an assumed functional form it is not obvious where in the temperature interval the coefficient of thermal expansion and the derivative of the material compliance should be evaluated. Here three different cases were considered. The coefficient of thermal expansion and the derivative of the compliance in eq. (B.3) being evaluated at the beginning of the temperature interval $\left(T_{n-1}\right)$, at the center of the temperature interval $\left(0.5\left(T_{n-1}+T_{n}\right)\right)$, and at the end of the temperature interval $\left(T_{n}\right)$. In Tables B.2 and B.3 the stresses in the beam are shown for the different cases for different sizes of the temperature increments and compared to the exact solution. As can be seen in Tables B.2 and B.3 a large number of increments needed to be used to obtain accurate stresses. The results were most accurate when the coefficient of thermal expansion and the derivative of the compliance were evaluated at the center of the temperature interval. Evaluating the coefficient of thermal expansion and the derivative of the compliance at the end or beginning of the temperature interval resulted in large errors for small numbers of increments.

Finally, the effect of the coupling term $\Delta S \sigma\left(T_{n-1}\right)$ in eq. (B.2) on the stresses was 


\section{APPENDIX B. COMPARISON OF INCREMENTAL CONSTITUTIVE EQUATIONS201}

Table B.2: Stresses calculated using the incremental constitutive equations based on an assumed functional form for 10 and 20 temperature increments.

\begin{tabular}{|c|c|c|c|c|c|c|c|}
\hline \multirow{3}{*}{$\mathrm{T}\left[{ }^{\circ} \mathrm{C}\right]$} & \multicolumn{7}{|c|}{$\sigma[\mathrm{MPa}]$} \\
\hline & \multirow{2}{*}{ Exact } & \multicolumn{3}{|c|}{10 increments $\left(\Delta T=100^{\circ} C\right)$} & \multicolumn{3}{|c|}{20 increments $\left(\Delta T=50^{\circ} \mathrm{C}\right)$} \\
\hline & & $T_{n-1}$ & $0.5\left(T_{n-1}+T_{n}\right)$ & $T_{n}$ & $T_{n-1}$ & $0.5\left(T_{n-1}+T_{n}\right)$ & $T_{n}$ \\
\hline 0 & 0.0 & 0.0 & 0.0 & 0.0 & 0.0 & 0.0 & 0.0 \\
\hline 100 & -185.9 & -320.6 & -171.4 & -130.9 & -251.1 & -180.2 & -149.2 \\
\hline 200 & -287.0 & -447.1 & -271.0 & -217.9 & -362.8 & -281.0 & -242.4 \\
\hline 300 & -355.4 & -515.5 & -339.6 & -283.8 & -431.2 & -349.5 & -309.9 \\
\hline 400 & -412.3 & -568.6 & -396.8 & -340.5 & -486.5 & -406.5 & -367.0 \\
\hline 500 & -464.5 & -618.5 & -449.2 & -392.4 & -537.8 & -458.7 & -419.3 \\
\hline 600 & -513.7 & -667.1 & -498.6 & -441.0 & -586.9 & -508.1 & -468.3 \\
\hline 700 & -560.4 & -714.2 & -545.4 & -486.7 & -633.8 & -554.8 & -514.6 \\
\hline 800 & -604.7 & -759.2 & -589.7 & -530.0 & -678.5 & -599.1 & -558.4 \\
\hline 900 & -646.8 & -802.2 & -631.7 & -571.0 & -721.1 & -641.1 & -600.0 \\
\hline 1000 & -686.8 & -843.1 & -671.7 & -610.0 & -761.5 & -681.1 & -639.5 \\
\hline
\end{tabular}

Table B.3: Stresses calculated using the incremental constitutive equations based on an assumed functional form for 100 and 1000 temperature increments.

\begin{tabular}{|r|r||r|r|r||r|r|r|}
\hline \multirow{2}{*}{$\mathrm{T}\left[{ }^{\circ} \mathrm{C}\right]$} & \multicolumn{6}{|c|}{$\sigma[\mathrm{MPa}]$} \\
\cline { 2 - 7 } & \multirow{2}{*}{ Exact } & \multicolumn{10}{|c|}{100 increments $\left(\Delta T=10^{\circ} C\right)$} & \multicolumn{1}{|c|}{1000 increments $\left(\Delta T=1^{\circ} C\right)$} \\
\cline { 3 - 7 } & & $T_{n-1}$ & $0.5\left(T_{n-1}+T_{n}\right)$ & $T_{n}$ & $T_{n-1}$ & $0.5\left(T_{n-1}+T_{n}\right)$ & $T_{n}$ \\
\hline 0 & 0.0 & 0.0 & 0.0 & 0.0 & 0.0 & 0.0 & 0.0 \\
100 & -185.9 & -197.5 & -185.6 & -175.8 & -187.0 & -185.9 & -184.8 \\
200 & -287.0 & -300.6 & -286.7 & -275.2 & -288.3 & -287.0 & -285.8 \\
300 & -355.4 & -369.0 & -355.1 & -343.5 & -356.8 & -355.4 & -354.2 \\
400 & -412.3 & -425.6 & -412.0 & -400.5 & -413.6 & -412.3 & -411.0 \\
500 & -464.5 & -477.6 & -464.1 & -452.8 & -465.7 & -464.5 & -463.2 \\
600 & -513.7 & -526.9 & -513.4 & -502.0 & -515.0 & -513.7 & -512.5 \\
700 & -560.4 & -573.7 & -560.1 & -548.7 & -561.7 & -560.4 & -559.2 \\
800 & -604.7 & -618.1 & -604.4 & -592.9 & -606.0 & -604.7 & -603.5 \\
900 & -646.8 & -660.2 & -646.5 & -634.8 & -648.1 & -646.8 & -645.5 \\
1000 & -686.8 & -700.3 & -686.4 & -674.7 & -688.1 & -686.8 & -685.5 \\
\hline
\end{tabular}


Table B.4: Effect of the coupling term on the stresses.

\begin{tabular}{|r|c|c|c|}
\hline \multirow{2}{*}{$\mathrm{T}\left[{ }^{\circ} \mathrm{C}\right]$} & \multicolumn{3}{|c|}{$\sigma[\mathrm{MPa}]$} \\
\cline { 3 - 4 } & \multirow{2}{*}{ eq. (B.2) } & \multicolumn{2}{|c|}{ Neglect coupling } \\
\cline { 3 - 4 } & & $\Delta T=100^{\circ} C$ & $\Delta T=10^{\circ} C$ \\
\hline 0 & 0.0 & 0.0 & 0.0 \\
100 & -185.9 & -185.9 & -178.8 \\
200 & -287.0 & -293.4 & -288.5 \\
300 & -355.4 & -374.3 & -371.1 \\
400 & -412.3 & -441.4 & -438.9 \\
500 & -464.5 & -499.9 & -497.8 \\
600 & -513.7 & -552.6 & -550.7 \\
700 & -560.4 & -601.0 & -599.2 \\
800 & -604.7 & -646.2 & -644.4 \\
900 & -646.8 & -688.6 & -686.8 \\
1000 & -686.8 & -728.7 & -726.9 \\
\hline
\end{tabular}

studied. For this, stresses were calculated using this incremental constitutive equation with and without the coupling term. In Table B.4 the stress is shown for the case with the incremental constitutive equations including the coupling term and for the case with the incremental constitutive equations without the coupling term for 10 temperature increments $\left(\Delta T=100^{\circ} C\right)$ and for 100 temperature increments $\left(\Delta T=10^{\circ} C\right)$. As can be seen in Table B.4, if the coupling term is neglected, the stress diverges with increasing temperature. Increasing the number of temperature increments improves the results only marginally. This clearly shows that the coupling term plays an important role in the incremental analysis with temperature-dependent properties even if only linear elastic materials are considered.

In summary, results calculated using the incremental constitutive equations based on the difference in stress states at two different temperatures (eq. (B.2)) are exact. Results calculated using the incremental constitutive equations based on an assumed functional form (eq. (B.3)) converge to the exact solution for a larger number of temperature increments. If these constitutive equations are used, the coefficient of thermal expansion and the derivative of the compliance should be evaluated at the center of the temperature intervals. Neglecting 
APPENDIX B. COMPARISON OF INCREMENTAL CONSTITUTIVE EQUATIONS203 the coupling term in the incremental constitutive equations causes divergence of the stresses with increasing temperature. 


\section{Appendix $\mathbf{C}$}

\section{Material Stiffness Matrix and its}

\section{Transformation}

For an orthotropic material, the material stiffness matrix in principal material directions, here denoted as the 1-2-3 coordinate system, is given by

$$
[C]=\left[\begin{array}{cccccc}
C_{11} & C_{12} & C_{13} & 0 & 0 & 0 \\
C_{12} & C_{22} & C_{23} & 0 & 0 & 0 \\
C_{13} & C_{23} & C_{33} & 0 & 0 & 0 \\
0 & 0 & 0 & C_{44} & 0 & 0 \\
0 & 0 & 0 & 0 & C_{55} & 0 \\
0 & 0 & 0 & 0 & 0 & C_{66}
\end{array}\right]
$$

where the material stiffness coefficients are

$$
\begin{array}{clrl}
C_{11} & =\frac{1-\nu_{23} \nu_{32}}{\Delta} E_{11} & C_{12}=\frac{\nu_{12}+\nu_{13} \nu_{32}}{\Delta} E_{22} & C_{13}=\frac{\nu_{13}+\nu_{12} \nu_{23}}{\Delta} E_{33} \\
C_{22}=\frac{1-\nu_{13} \nu_{31}}{\Delta} E_{22} & C_{23}=\frac{\nu_{23}+\nu_{21} \nu_{13}}{\Delta} E_{33} & C_{33}=\frac{1-\nu_{12} \nu_{21}}{\Delta} E_{33} \\
C_{44}=G_{23} & C_{55}=G_{13} & C_{66}=G_{12} \\
\Delta=1-\nu_{12} \nu_{21}-\nu_{13} \nu_{31}-\nu_{23} \nu_{32}-\nu_{12} \nu_{23} \nu_{31}-\nu_{21} \nu_{13} \nu_{32} .
\end{array}
$$




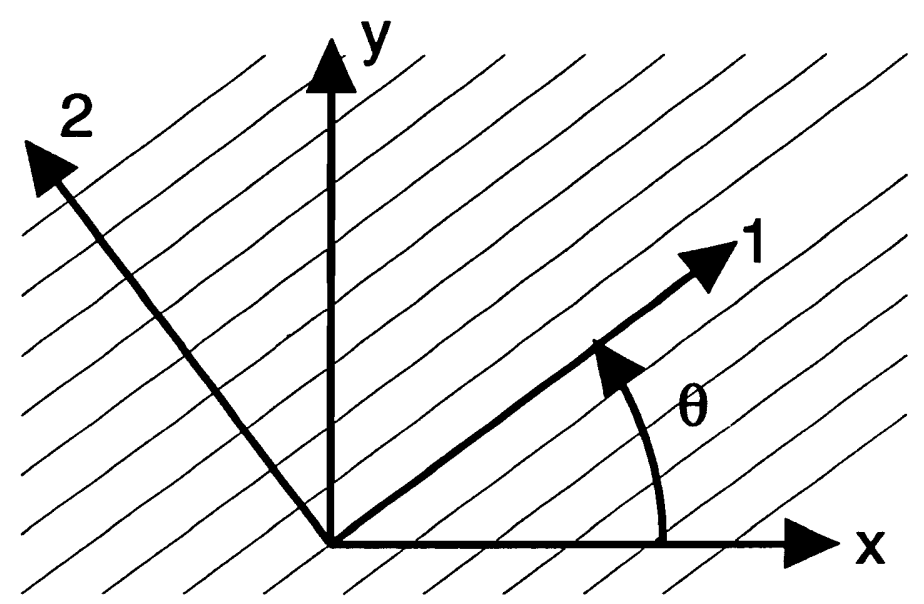

Figure C.1: Rotation from a $x-y-z$ global coordinate system to the 1-2-3 material coordinate system.

The transformation from the material coordinate system (1-2-3) to another coordinate system, here denoted by $x-y-z$, is given by

$$
[\bar{C}]=\left[T_{1}\right]^{-1}[C]\left[T_{2}\right]
$$

where the transformation matrices $\left[T_{1}\right]$ and $\left[T_{2}\right]$ for a rotation $\theta$ about the $z$-axis as indicated in Fig. C.1 are given by

$$
\left[T_{1}\right]=\left[\begin{array}{cccccc}
m^{2} & n^{2} & 0 & 0 & 0 & 2 m n \\
n^{2} & m^{2} & 0 & 0 & 0 & -2 m n \\
0 & 0 & 1 & 0 & 0 & 0 \\
0 & 0 & 0 & m & -n & 0 \\
0 & 0 & 0 & n & m & 0 \\
-m n & m n & 0 & 0 & 0 & m^{2}-n^{2}
\end{array}\right]
$$


APPENDIX C. MATERIAL STIFFNESS MATRIX AND ITS TRANSFORMATION206

$$
\left[T_{2}\right]=\left[\begin{array}{cccccc}
m^{2} & n^{2} & 0 & 0 & 0 & m n \\
n^{2} & m^{2} & 0 & 0 & 0 & -m n \\
0 & 0 & 1 & 0 & 0 & 0 \\
0 & 0 & 0 & m & -n & 0 \\
0 & 0 & 0 & n & m & 0 \\
-2 m n & 2 m n & 0 & 0 & 0 & m^{2}-n^{2}
\end{array}\right]
$$

where

$$
m=\cos \theta \quad n=\sin \theta .
$$

Carrying out the matrix multiplications in eq. (C.3) results in the following transformation equations for the material stiffnesses:

$$
\begin{aligned}
& \bar{C}_{11}=C_{11} m^{4}+2\left(C_{12}+2 C_{66}\right) m^{2} n^{2}+C_{22} n^{4} \\
& \bar{C}_{12}=\left(C_{11}+C_{22}-4 C_{66}\right) m^{2} n^{2}+C_{12}\left(m^{4}+n^{4}\right) \\
& \bar{C}_{13}=C_{13} m^{2}+C_{23} n^{2} \\
& \bar{C}_{16}=\left(\left(C_{11}-C_{12}-2 C_{66}\right) m^{2}+\left(C_{12}-C_{22}+2 C_{66}\right) n^{2}\right) m n \\
& \bar{C}_{22}=C_{11} n^{4}+2\left(C_{12}+2 C_{66}\right) m^{2} n^{2}+C_{22} m^{4} \\
& \bar{C}_{23}=C_{13} n^{2}+C_{23} m^{2} \\
& \bar{C}_{26}=\left(\left(C_{11}-C_{12}-2 C_{66}\right) n^{2}+\left(C_{12}-C_{22}+2 C_{66}\right) m^{2}\right) m n \\
& \bar{C}_{33}=C_{33} \\
& \bar{C}_{36}=\left(C_{13}-C_{23}\right) m n \\
& \bar{C}_{44}=C_{44} m^{2}+C_{55} n^{2} \\
& \bar{C}_{45}=\left(C_{55}-C_{44}\right) m n \\
& \bar{C}_{55}=C_{44} n^{2}+C_{55} m^{2} \\
& \bar{C}_{66}=\left(C_{11}-2 C_{12}+C_{22}\right) m^{2} n^{2}+C_{66}\left(m^{2}-n^{2}\right)^{2} \\
& \bar{C}_{14}=\bar{C}_{15}=\bar{C}_{24}=\bar{C}_{25}=\bar{C}_{34}=\bar{C}_{35}=\bar{C}_{46}=\bar{C}_{56}=0
\end{aligned}
$$




\section{APPENDIX C. MATERIAL STIFFNESS MATRIX AND ITS TRANSFORMATION207}

Note that the expressions for the transformations for $\bar{C}_{44}, \bar{C}_{45}$, and $\bar{C}_{55}$ are given here only for completeness. These terms do not appear in the derivation of the governing equations for the finite element model since shear responses in the $y z$ and $x z$ planes do not occur for the current problem. 


\section{Appendix D}

\section{Implementation of the Finite}

\section{Element Model}

While the finite element Eqs. (2.32) are general, the implementation of the finite element model is specific to the problem of interest shown in Fig. 2.1.

\section{D.1 Mesh Generation}

A special purpose mesh generator and a preprocessor for the finite element program were developed. The main parameters for the mesh generation are the width, height, and corner radius of the substrate, the number of coatings and their respective thicknesses, and the minimum number of elements through the thickness of a coating. Usually more than one element is required through the thickness of a coating to obtain converged results. The generated mesh consists of 8-node quadrilateral and 6-node triangular isoparametric elements. All coatings are modeled with 8-node elements for convergence purposes. A sample mesh is shown in Fig. D.1. A bandwidth optimizer based on the algorithm by Gibbs, Poole and Stockmeyer [16] was implemented to reduce the problem size. The preprocessor generates and optimizes the mesh for the geometry of the substrate with all coatings applied to the substrate. In the analysis sequence as indicated in Fig. 2.1, almost-zero properties are as- 


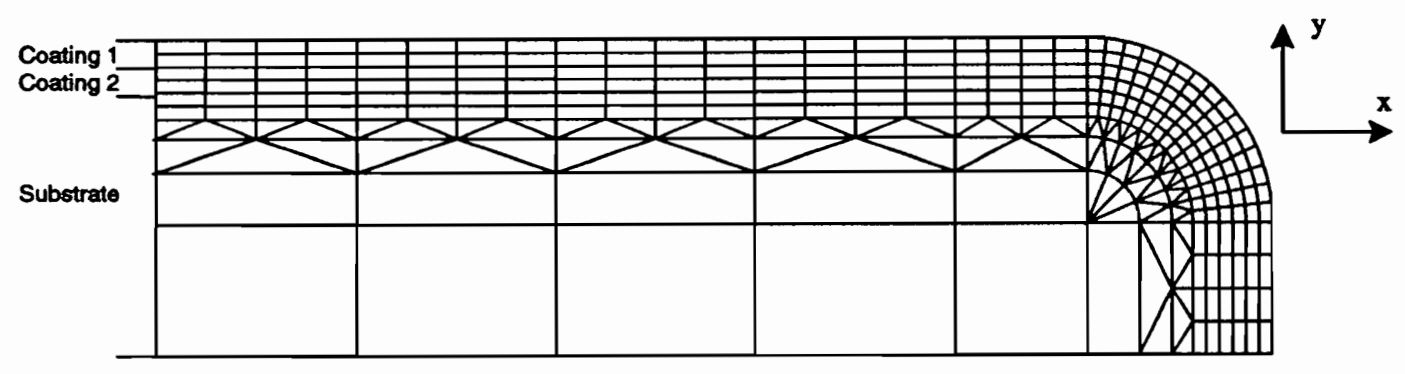

Figure D.1: Example of a mesh generated by the preprocessor.

signed to elements that correspond to coatings that are in reality not yet applied to the composite. This results in zero stresses in these elements and thus is in accordance with the assumption that the coatings are stress free when they are applied. Using the same mesh for all analysis steps has the advantage that the nodal points and Gauss points of the elements for all analysis steps are at the same locations. Generating a new mesh each time a coating is added to the composite would require a substantial amount of bookkeeping.

\section{D.2 Material Data Input}

\section{D.2.1 Evaluation of Temperature-Dependent Material Properties}

The material data for each property needs to be input in tabular form. For each property the value is specified at a number of temperatures. To obtain properties at other than the specified temperatures, linear interpolation is used. For temperatures higher than the largest given temperature, the property value is assumed to be the value at the highest given temperature. Similarly, for temperatures lower than the lowest given temperature, the value of the property is assumed to be the value at the lowest temperature. Thus, all properties are represented by a piecewise linear function.

Thermal strains are calculated by integrating the piecewise linear function for the coefficients of thermal expansion from a given reference temperature to the temperature at which the thermal strain is needed. This results in a piecewise quadratic representation of 
the free thermal strains.

\section{D.2.2 Evaluation of Gradient Layer Properties}

It is assumed that the material properties vary linearly through the thickness of a gradient coating. More complex property variations need to be approximated by dividing the gradient coating into several smaller layers with linear property variations. For each gradient coating two material data sets are required, one for the outer surface of the coating and one for the inner surface of the coating. Properties within the coating are obtained by linearly interpolating between these property sets.

To obtain properties in a gradient coating at a given temperature and location through the thickness, the property sets for the two surfaces of the gradient coating are first evaluated at the given temperature and then linear interpolation through the thickness is applied.

\section{D.3 Numerical Intergration of Shape Functions of Finite El- ements}

The elements available in the finite element program are the 6-node triangular isoparametric element and the 8-node quadrilateral isoparametric element. The geometries of the elements along with the node numbering and Gauss point numbering schemes are illustrated in Fig. D.2. The shape functions for the 6-node triangular element are:

$$
\begin{aligned}
& \psi_{1}=2 \xi^{2}-3 \xi+4 \xi \eta-3 \eta+2 \eta^{2}+1 \\
& \psi_{2}=2 \xi^{2}-\xi \\
& \psi_{3}=2 \eta^{2}-\eta \\
& \psi_{4}=-4 \xi^{2}+4 \xi-4 \xi \eta \\
& \psi_{5}=4 \xi \eta \\
& \psi_{6}=-4 \eta^{2}+4 \eta-4 \xi \eta
\end{aligned}
$$




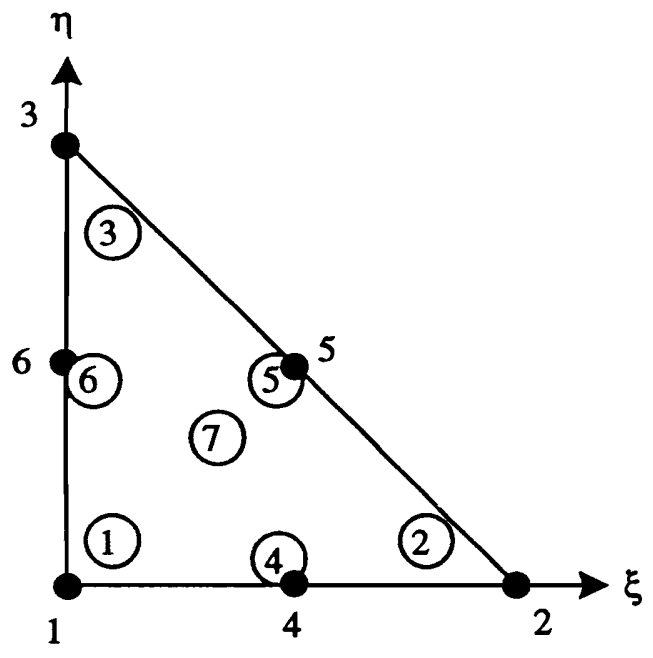

Element local node number

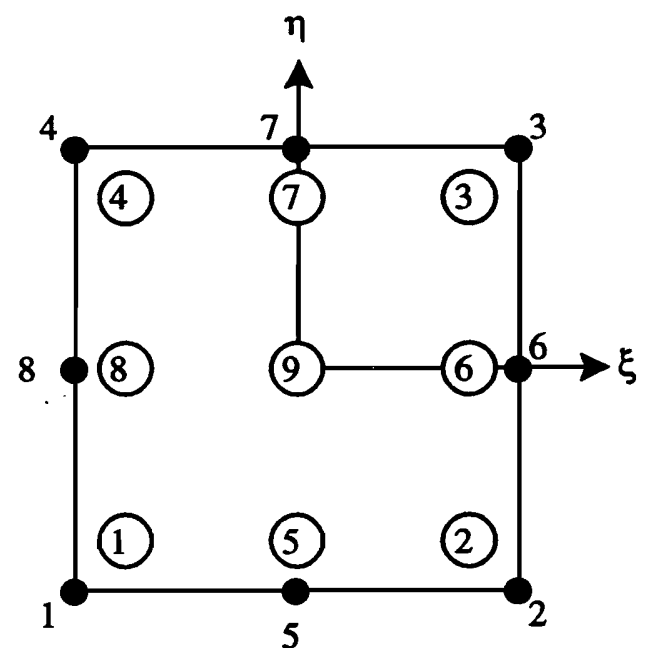

(n) Location of Gauss point $\mathbf{n}$

Figure D.2: Available element geometries.

The shape functions for the quadrilateral element are:

$$
\begin{aligned}
& \psi_{1}=0.25(1-\xi)(1-\eta)(-1-\xi-\eta) \\
& \psi_{2}=0.25(1+\xi)(1-\eta)(-1+\xi-\eta) \\
& \psi_{3}=0.25(1+\xi)(1+\eta)(-1+\xi+\eta) \\
& \psi_{4}=0.25(1-\xi)(1+\eta)(-1-\xi+\eta) \\
& \psi_{5}=0.50\left(1-\xi^{2}\right)(1-\eta) \\
& \psi_{6}=0.50(1+\xi)\left(1-\eta^{2}\right) \\
& \psi_{7}=0.50\left(1-\xi^{2}\right)(1+\eta) \\
& \psi_{8}=0.50(1-\xi)\left(1-\eta^{2}\right)
\end{aligned}
$$

In the standard numerical integration scheme material properties are calculated for an element and therefore are the same at each Gauss point. This results in accurate models if material properties do not vary within an element. In the present study, however, material properties for orthotropic coatings or in gradient coatings may actually vary significantly 
within a finite element. Therefore, to obtain more accurate models, the material stiffnesses $\bar{C}_{i j k l}$ are evaluated at each Gauss point for elements in the corner region and for elements in gradient coatings. In the following "special numerical integration" refers to numerical integration with material stiffness being evaluated at each Gauss point. That evaluating the material stiffnesses at each Gauss point in fact results in more accurate models was shown by Avery and Herakovich [8] for an axisymmetric geometry. In Fig. 4.7 it can be seen that the special integration results in more accurate stresses in gradient coatings.

In the present research the finite element model is implemented so that it is automatically detected if standard or special numerical integration should be used. For this purpose, each element is assigned two flags that indicates whether or not the element is in a gradient coating and whether or not the element is in the coatings in the corner region. If an element is in a gradient coating or an element is in the corner region, special numerical integration is used. All elements in the substrate can be integrated using standard numerical integration, because the 1-2-3 material coordinate system and the global $x-y-z$ coordinate system are aligned for the substrate. Several geometric parameters are used to automatically calculate properties and material stiffnesses at each Gauss point for the special numerical integration. These parameters are indicated in Fig. D.3. In Fig. D.3 a quarter model of the substrate with several coatings is shown. The coordinates $x_{0}$ and $y_{0}$ in the figure represent the origin of the global $x-y-z$ coordinate system and are normally taken as $(0,0)$. The coordinates $x_{R}$ and $y_{R}$ represent the origin of the corner radius, and the coordinates $x_{C}$ and $y_{C}$ are reference coordinates for a coating.

\section{D.3.1 Properties at Gauss Points in a Gradient Coating}

As was mentioned earlier, linear interpolation is used to calculate material properties within a gradient coating. Properties at Gauss points in elements are calculated using

$$
\mathcal{P}_{g p}=\mathcal{P}_{1}+\frac{\mathcal{P}_{2}-\mathcal{P}_{1}}{t_{g p}-t_{g}}
$$




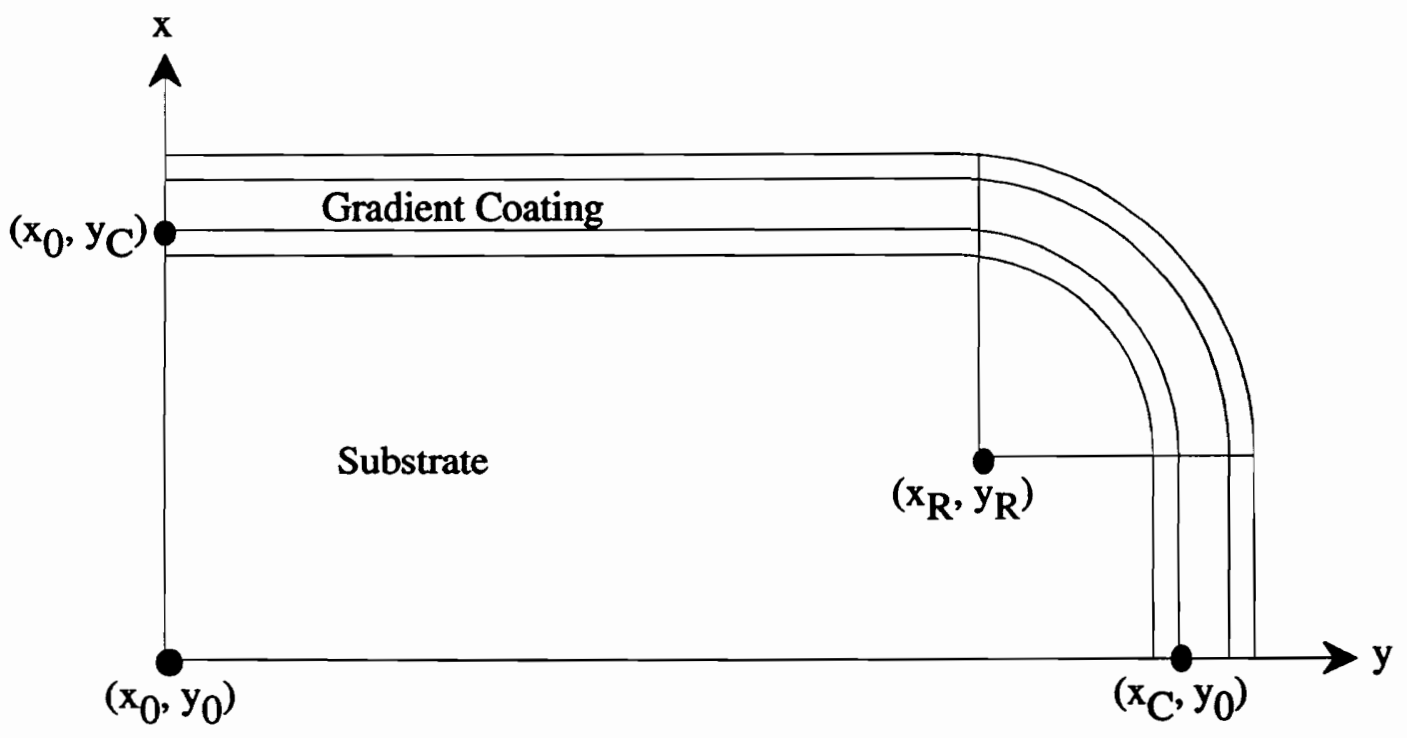

Figure D.3: Parameters used for special numerical integration.

where, as indicated in Fig. D.4, $t_{g}$ is the thickness of the gradient coating, $t_{g p}$ is the distance from the inner surface of the gradient coating to the Gauss point, $\mathcal{P}_{g p}$ is a material property at the Gauss point, $\mathcal{P}_{1}$ is a material property on the inner surface of the gradient coating, and $\mathcal{P}_{2}$ is a material property at the outer surface of the gradient coating. The properties $\mathcal{P}_{1}$ and $\mathcal{P}_{2}$ and the thickness $t_{g}$ are input parameters to the finite element location. The distance $t_{g p}$ is calculated depending on the location of the element in the gradient coating. If $x_{g p}$ and $y_{g p}$ are the global coordinates of a Gauss point, then if the element is to the left of the corner in Fig. D.3, then

$$
t_{g p}=y_{g p}-y_{C},
$$

if the element is below the corner, then

$$
t_{g p}=x_{g p}-x_{C},
$$




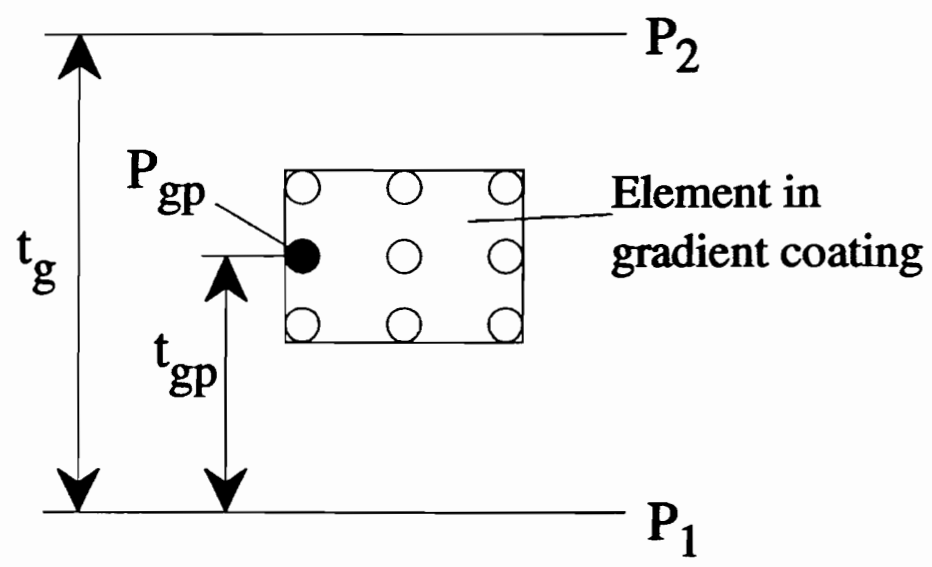

Figure D.4: Calculation of properties in a gradient coating.

and if the element is in the corner, then

$$
t_{g p}=\sqrt{\left(x_{g p}-x_{R}\right)^{2}+\left(y_{g p}-y_{R}\right)^{2}}-x_{C}
$$

\section{D.3.2 Transformation Angle in Corner Region}

In Fig. D.5 a finite element in the corner region is drawn. The Gauss points, the material coordinate system, and the global coordinate system are indicated. The transformation angle $\theta$ indicated in Fig. C.1 can be calculated using the global coordinates $x_{g p}$ and $y_{g p}$ of the Gauss point and the coordinates of the origin of the corner $x_{R}$ and $y_{R}$. First, an intermediate angle $\theta_{1}$ is calculated. This angle is

$$
\theta_{1}=\tan ^{-1}\left(\frac{y_{g p}-y_{R}}{x_{g p}-x_{R}}\right)
$$

This angle is actually the angle between the global $x$ axis and the material 2 -axis. Thus, the angle between the global $x$-axis and the material 1-axis that is used in the transformation is

$$
\theta=\theta_{1}-\Pi / 2
$$




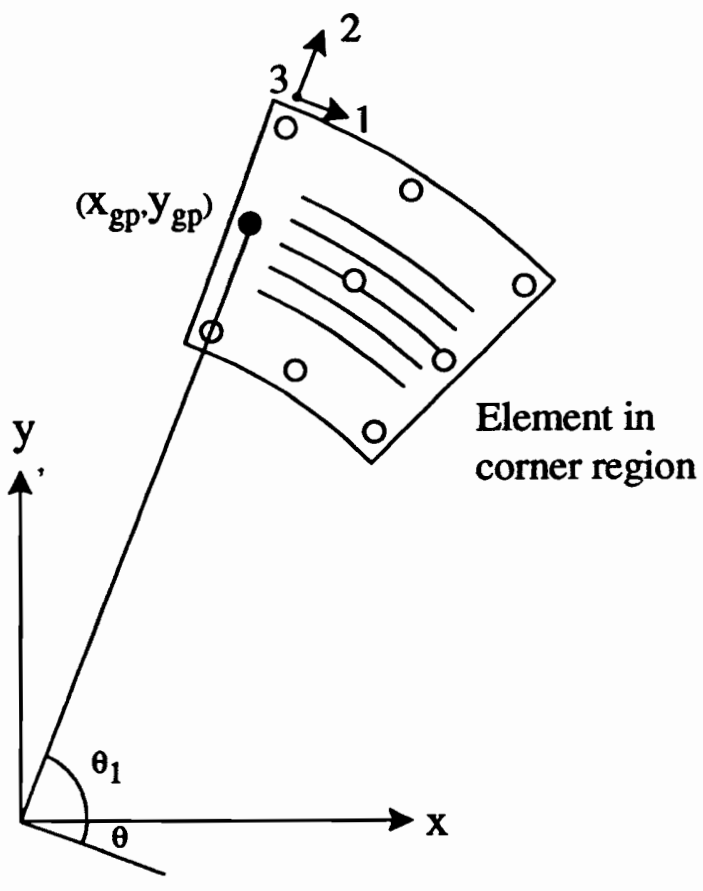

$\left(\mathbf{X}_{\mathrm{R}}, \mathbf{Y}_{\mathbf{R}}\right)$

Figure D.5: Calculation of transformation angle.

\section{D.4 Solution Procedure for the Finite Element Program}

The following is a brief outline of how the finite element analysis procedure works and the sequence of steps used:

1. Begin at some reference temperature. All stresses and strains are assumed to be zero.

2. Select the temperature increment.

3. Evaluate the material properties at the given temperatures.

4. Calculate the element stiffness matrix and equivalent thermal load vector. Note that the equivalent thermal load vector depends on the stresses calculated in the previous temperature step. 
5. Assemble the global stiffness matrix and equivalent thermal load vector.

6. Apply the boundary conditions.

7. Solve the system of equations for $\{\Delta u\},\{\Delta v\}$, and $\Delta \epsilon_{z}$.

8. Calculate the stress increments from eq. (2.17).

9. Compute the total stress $\{\sigma\}=\{\sigma\}+\{\Delta \sigma\}$.

10. Repeat steps 2 to 9 for all temperature increments.

The temperature increment in step 2 is calculated by the preprocessor. In the input data set, the application temperatures of the coatings and the desired analysis temperatures need to be input.

\section{D.5 Special Case: Temperature-Independent Analysis}

If temperature-dependence of the material properties is neglected, the finite element model is significantly simplified. For temperature-independent material properties, the coupling term $\Delta S_{k l r s} \sigma_{r s}$ in the incremental constitutive equations (eq. (2.17)) is zero. This means that the stresses calculated for any temperature increment are independent of the stresses existing in the composite at the beginning of the increment. Therefore simple superposition of several sub-analyses, one for each temperature increment, can be used to obtain the stresses at a desired temperature. For example, if temperature-independent properties were assumed for the problem shown in Fig. 2.1, the procedure for the finite element analysis would be as follows:

1. Use a temperature increment $\Delta T$ in the analysis so that the substrate is heated from its reference state to $1000^{\circ} \mathrm{C}$. Save the calculated strains. Note that at this point the substrate is stress free because of free thermal expansion. This step can be omitted if the thermal strains are not of interest. 
2. Apply a $\Delta T$ of $500^{\circ} \mathrm{C}$ to a model of the substrate and the $\mathrm{B}_{4} \mathrm{C}$ coating, and calculate the strains and stresses. Save these results.

3. Apply a $\Delta T$ of $-500^{\circ} \mathrm{C}$ to a model of the substrate and the two coatings, and calculate strains and stresses. Save these results.

4. Superpose all calculated results to obtain the actual state of stress in substrate and coatings at $1000^{\circ} \mathrm{C}$.

This form of the temperature-independent analysis was used to verify the incremental finite element model. 


\section{VITA}

The author was born on May 5, 1965 in Hannover, Germany. He graduated from the Christophorus Gymnasium in Braunschweig, Germany, in June 1985. After completing his military service in September 1986, he began his engineering studies at the TU Braunschweig, Germany, where he received the Vordiplom in October 1988. Between October 1988 and August 1989 he studied aerospace engineering at the TU Braunschweig. In September 1989 he transferred to the Department of Aerospace and Ocean Engineering at Virginia Polytechnic Institute and State University, USA, where he completed his studies towards the Master of Science in December 1991 with the thesis entitled, "Nonlinear Static and Transient Analysis of Generally Laminated Composite Beams." He then joined the Department of Engineering Science and Mechanics at Virginia Polytechnic Institute and State University to pursue a Ph.D., which he completed in April 1995. In May 1995 he will start a postdoctoral position at the Center for Composite Materials at the University of Delaware.

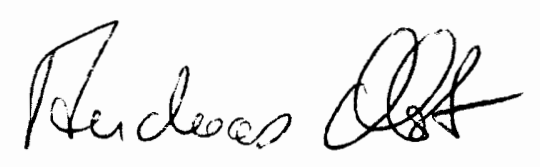

